\title{
Synthesis of Highly Substituted Phenols and Benzenes with Complete Regiochemical Control
}

\author{
Xiaojie Zhang, Christopher M. Beaudry* \\ Department of Chemistry, Oregon State University, Corvallis OR 97331

\section{Supporting Information}

Table of Contents

$\begin{array}{ll}\text { 1. General Experimental Details } & \text { S002 }\end{array}$

2. Preparation of Pyrone Coupling Partners (22) S003

3. Preparation of Nitroalkene Coupling Partners (23) S008

$\begin{array}{lr}\text { 4. Syntheses of Substituted Phenols } & \text { S012 }\end{array}$

$\begin{array}{ll}\text { 5. Syntheses of Substituted Benzenes } & \text { S033 }\end{array}$

$\begin{array}{ll}\text { 6. Reference } & \text { S038 }\end{array}$

$\begin{array}{ll}\text { 7. Depicted }{ }^{1} \mathrm{H} \text { and }{ }^{13} \mathrm{C} \text { Spectra } & \text { S040 }\end{array}$

$\begin{array}{lr}\text { 8. GC Data } & \text { S171 }\end{array}$ 


\section{General Experimental Details:}

All reactions were carried out under an inert Ar atmosphere in oven-dried glassware with magnetic stirring. Unless otherwise noted, reactions were heated using an oil bath, and reaction temperatures refer to external bath temperatures. Flash column chromatography (FCC) was carried out with SiliaFlash P60, $60 \AA$ silica gel. Reactions and column chromatography were monitored with EMD silica gel 60 F254 plates and visualized with potassium permanganate stain.

Reagent grade 1,4-dioxane was dried over calcium hydride and distilled prior to use. 1,2-Dichlorobenzene was distilled under reduce pressure and degassed using three freeze-pump-thaw cycles. All other reagents and solvents were used without further purification from commercial sources. Unless otherwise noted, melting points were obtained from material that solidified after chromatography.

Instrumentation: FT-IR spectra were obtained on $\mathrm{NaCl}$ plates with a PerkinElmer Spectrum Vision spectrometer. Microwave reactions were performed with a Biotage ${ }^{\circledR}$ Initiator $^{+}$Microwave System. HRMS were recorded on a JEOL MS Route Magnetic Sector Instrument (EI) or a Waters Synapt HDMS TOF instrument (ESI). Unless otherwise noted, proton and carbon NMR spectra $\left({ }^{1} \mathrm{H}\right.$ NMR and $\left.{ }^{13} \mathrm{C} \mathrm{NMR}\right)$ were recorded in deuterated chloroform $\left(\mathrm{CDCl}_{3}\right)$ on a Bruker $700 \mathrm{MHz}$ Avance III Spectrometer with s carbon-optimized cryoprobe or a Bruker $400 \mathrm{MHz}$ DPX-400 spectrometer. Multiplicities are abbreviated as follows: $\mathrm{s}=\operatorname{singlet}, \mathrm{d}=\operatorname{doublet}, \mathrm{t}=$ triplet, $\mathrm{q}=$ quartet, pent $=$ pentet, sext $=$ sextet, $\mathrm{sept}=$ septet, $\mathrm{br}=$ broad, $\mathrm{m}=$ multiplet . Melting points were determined with a Cole-Parmer instrument and are uncorrected. 


\section{Preparation of Pyrone Coupling Partners}

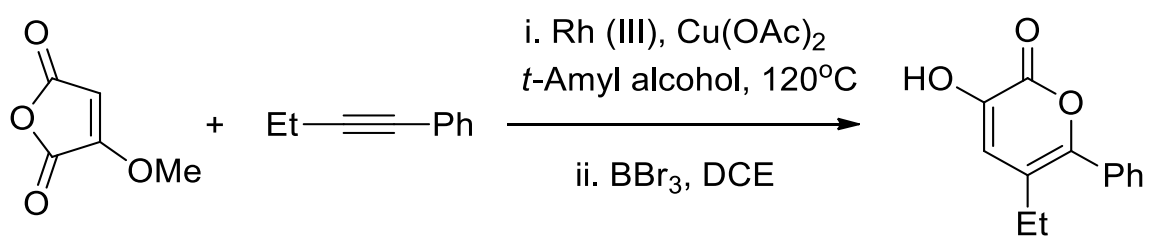

\section{5-Ethyl-3-hydroxy-6-phenyl-2H-pyran-2-one (22-S1)}

To a thick-walled reaction vessel were added a solution of 3-methoxyfuran-2,5-dione (1.98 g, $15.5 \mathrm{mmol}, 1.5 \mathrm{eq})$ in $t$-amyl alcohol $(100 \mathrm{~mL})$, tris(acetonitrile)penta methylcyclopentadienylrhodium(III) hexafluoroantimonate $(17 \mathrm{mg}, 0.021 \mathrm{mmol}$, $0.002 \mathrm{eq}), \mathrm{Cu}(\mathrm{OAc})_{2}(2.82 \mathrm{~g}, 15.5 \mathrm{mmol}, 1.5 \mathrm{eq})$ and 1-phenyl-butyne (1.34 g, 10.3 mmol, 1 eq). The headspace of the vessel was evacuated by brief exposure to vacuum, and the vessel was back-filled with Ar. The vessel was evacuated and back-filled with Ar two additional times. The tube was quickly sealed and heated to $120^{\circ} \mathrm{C}$ for $16 \mathrm{~h}$. The reaction mixture was cooled to $\mathrm{rt}$ and filtered through Celite with EtOAc to remove solids. The filtrate was concentrated. The residue was purified by FCC (5:1 hexanes:EtOAc) to yield 5-ethyl-3-methoxy-6-phenyl-2H-pyran-2-one as a white solid (1.76 g, 74\%).

Data for 5-ethyl-3-methoxy-6-phenyl-2H-pyran-2-one: Rf: 0.37 (3:2 hexanes:EtOAc); mp: $111-115{ }^{\circ} \mathrm{C}$; IR (thin film) 1726, $1639 \mathrm{~cm}^{-1} ;{ }^{1} \mathrm{H}$ NMR $\left(700 \mathrm{MHz}, \mathrm{CDCl}_{3}\right) \delta 7.43$ $(\mathrm{t}, J=7.0 \mathrm{~Hz}, 2 \mathrm{H}), 7.38(\mathrm{t}, J=7.7 \mathrm{~Hz}, 1 \mathrm{H}), 7.27-7.26(\mathrm{~m}, 2 \mathrm{H}), 6.47(\mathrm{~s}, 1 \mathrm{H}), 3.80$ (s, $3 \mathrm{H}), 2.47(\mathrm{q}, J=7.7 \mathrm{~Hz}, 2 \mathrm{H}), 1.20(\mathrm{t}, J=7.0 \mathrm{~Hz}, 3 \mathrm{H}) ;{ }^{13} \mathrm{C} \mathrm{NMR}\left(176 \mathrm{MHz}, \mathrm{CDCl}_{3}\right) \delta$ $154.3,143.5,136.5,128.9,128.8,127.9,117.3,116.5,56.1,28.7,24.1,12.5$; HRMS (ESI) $\mathrm{m} / \mathrm{z}:[\mathrm{M}+\mathrm{H}]^{+}$Calcd for $\mathrm{C}_{14} \mathrm{H}_{15} \mathrm{O}_{3} 231.1021$; Found 231.1015 .

To a solution of 5-ethyl-3-methoxy-6-phenyl-2H-pyran-2-one $(1.67 \mathrm{~g}, 7.26 \mathrm{mmol}, 1$ eq) in 1,2-dichloroethane $(73 \mathrm{~mL}, 0.1 \mathrm{M})$ was added $\mathrm{BBr}_{3}(1 \mathrm{M}$ in heptane, $11 \mathrm{~mL}$, $11.0 \mathrm{mmol}, 1.5 \mathrm{eq})$ dropwise. The mixture was stirred at $\mathrm{rt}$ for $20 \mathrm{~min}$. The reaction was quenched by addition of sat'd aqueous $\mathrm{NaHCO}_{3}(100 \mathrm{~mL})$ and extracted with $\mathrm{CH}_{2} \mathrm{Cl}_{2}$ (4 x $30 \mathrm{~mL}$ ). The combined organic layers were dried over $\mathrm{Na}_{2} \mathrm{SO}_{4}$, filtered, and concentrated. The residue was purified by FCC (5:1 hexanes:EtOAc) to yield 22-S1 as a light yellow solid.

Data for 22-S1: Rf: 0.43 (2:1 hexanes:EtOAc); mp: $113-115{ }^{\circ} \mathrm{C}$; IR (thin film) 3309, 1683, $1651 \mathrm{~cm}^{-1}$; ${ }^{1} \mathrm{H}$ NMR $\left(700 \mathrm{MHz}, \mathrm{CDCl}_{3}\right) \delta 7.48-7.40(\mathrm{~m}, 5 \mathrm{H}), 6.76(\mathrm{~s}, 1 \mathrm{H})$, $6.24(\mathrm{~s}, 1 \mathrm{H}), 2.46(\mathrm{q}, J=7.7 \mathrm{~Hz}, 2 \mathrm{H}), 1.19(\mathrm{t}, J=7.7 \mathrm{~Hz}, 3 \mathrm{H}) ;{ }^{13} \mathrm{C} \mathrm{NMR}(176 \mathrm{MHz}$, $\left.\mathrm{CDCl}_{3}\right) \delta 161.3,148.7,140.8,132.2,129.3,128.6,128.4,118.8,118.5,23.4,14.5$; HRMS (ESI) m/z: [M + H $]^{+}$Calcd for $\mathrm{C}_{13} \mathrm{H}_{13} \mathrm{O}_{3}$ 217.0865; Found 217.0868. 


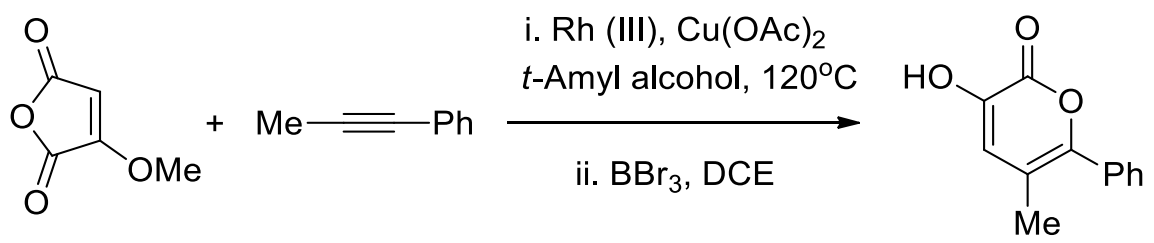

\section{3-Hydroxy-5-methyl-6-phenyl-2H-pyran-2-one (22-S2)}

To a thick-walled reaction vessel were added a solution of 3-methoxyfuran-2,5-dione (3.84 g, $30.0 \mathrm{mmol}, 1.5 \mathrm{eq})$ in $t$-amyl alcohol $(200 \mathrm{~mL})$, tris(acetonitrile)penta methylcyclopentadienylrhodium(III) hexafluoroantimonate $(33 \mathrm{mg}, 0.04 \mathrm{mmol}, 0.004$ eq), $\mathrm{Cu}(\mathrm{OAc})_{2}(5.46 \mathrm{~g}, 30.0 \mathrm{mmol}, 1.5 \mathrm{eq})$ and 1-phenyl-propyne (2.32 g, $20.0 \mathrm{mmol}$, 1 eq). The headspace of the vessel was evacuated by brief exposure to vacuum, and the vessel was back-filled with Ar. The vessel was evacuated and back-filled with Ar two additional times. The tube was quickly sealed and heated to $120^{\circ} \mathrm{C}$ for $16 \mathrm{~h}$. The reaction mixture was cooled to rt and filtered through Celite with EtOAc to remove solids. The filtrate was dried over $\mathrm{Na}_{2} \mathrm{SO}_{4}$, filtered, and concentrated. The residue was purified by FCC (3:1 hexanes:EtOAc) to yield 3-methoxy-5-methyl-6-phenyl-2Hpyran-2-one as a white solid (1.56 g, 36\%). Spectroscopic data for 3-methoxy-5-methyl-6-phenyl-2H- pyran-2-one matched those previously reported. ${ }^{1}$

To a solution of 3-methoxy-5-methyl-6-phenyl-2H-pyran-2-one (1.55 g, $5.5 \mathrm{mmol}, 1$ eq) in 1,2-dichloroethane $(72 \mathrm{~mL}, 0.1 \mathrm{M})$ was added $\mathrm{BBr}_{3}(1 \mathrm{M}$ in heptane, $8.6 \mathrm{~mL}$, $8.6 \mathrm{mmol}, 1.5 \mathrm{eq})$ dropwise. The mixture was stirred at $\mathrm{rt}$ for $20 \mathrm{~min}$. The reaction was quenched by addition of sat'd aqueous $\mathrm{NaHCO}_{3}(100 \mathrm{~mL})$ and extracted with $\mathrm{CH}_{2} \mathrm{Cl}_{2}$ (4 x $30 \mathrm{~mL}$ ). The combined organic layers were dried over $\mathrm{Na}_{2} \mathrm{SO}_{4}$, filtered, and concentrated. The residue was purified by FCC (3:1 hexanes:EtOAc) to yield 22S2 as a white solid (1.37 g, 97\%).

Data for 22-S2: $\mathrm{R}_{\mathrm{f}}: 0.54$ (pure $\mathrm{Et}_{2} \mathrm{O}$ ); mp: $134-138{ }^{\circ} \mathrm{C}$; IR (thin film) 3326, 1684, 1649, 1422, $1362 \mathrm{~cm}^{-1} ;{ }^{1} \mathrm{H}$ NMR $\left(700 \mathrm{MHz}, \mathrm{CDCl}_{3}\right) \delta 7.52(\mathrm{~d}, J=7.7 \mathrm{~Hz}, 2 \mathrm{H}), 7.44$ (t, $J=7.7 \mathrm{~Hz}, 2 \mathrm{H}), 7.41(\mathrm{t}, J=7.7 \mathrm{~Hz}, 1 \mathrm{H}), 6.69(\mathrm{~s}, 1 \mathrm{H}), 5.98(\mathrm{~s}, 1 \mathrm{H}), 2.18(\mathrm{~s}, 3 \mathrm{H})$; ${ }^{13} \mathrm{C} \mathrm{NMR}\left(176 \mathrm{MHz}, \mathrm{CDCl}_{3}\right) \delta 161.4,148.6,140.3,132.3,129.2,128.6,128.4,120.1$, 112.7, 17.4; HRMS (ESI) m/z: $[\mathrm{M}+\mathrm{H}]^{+}$Calcd for $\mathrm{C}_{12} \mathrm{H}_{11} \mathrm{O}_{3}$ 203.0708; Found 203.0709 .

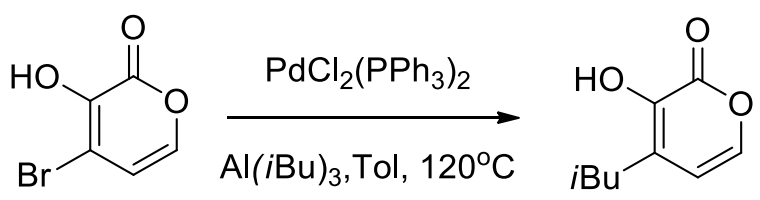

\section{3-Hydroxy-4-isobutyl-2H-pyran-2-one (22-S3)}

To a thick-walled reaction vessel were added a solution of 4-bromo-3-hydroxy2H-pyran-2-one (1.20 g, $6.3 \mathrm{mmol}, 1 \mathrm{eq})$ in toluene $(13 \mathrm{~mL}, 0.5 \mathrm{M})$ and $\mathrm{PdCl}_{2}\left(\mathrm{PPh}_{3}\right)_{2}$ (22 mg, $0.0315 \mathrm{mmol}, 0.005 \mathrm{eq}$ ). The mixture was stirred for $1 \mathrm{~min}$, and triisobutyl aluminum (1.1 $\mathrm{M}$ in toluene, $6.8 \mathrm{~mL}, 7.5 \mathrm{mmol}, 1.2 \mathrm{eq}$ ) was added dropwise. The tube was quickly sealed and heated to $100{ }^{\circ} \mathrm{C}$ for $4 \mathrm{~h}$. The reaction was cooled to rt, 
quenched with $5 \%$ citric acid $(30 \mathrm{~mL})$, and extracted with EtOAc $(5 \times 30 \mathrm{~mL})$. The combined organic layers were dried over $\mathrm{Na}_{2} \mathrm{SO}_{4}$, filtered, and concentrated. The residue was purified by FCC (5:1 hexanes:EtOAc) to yield 22-S3 as a yellow solid (538.1 mg, $51 \%)$.

Data for 22-S3: Rf: 0.54 (5:3 hexanes:EtOAc); mp: $39-41{ }^{\circ} \mathrm{C}$; IR (thin film) 3356, 2960, 1756, 1695, $1674 \mathrm{~cm}^{-1} ;{ }^{1} \mathrm{H}$ NMR $\left(700 \mathrm{MHz}, \mathrm{CDCl}_{3}\right) \delta 7.09(\mathrm{~d}, J=4.9 \mathrm{~Hz}, 1 \mathrm{H})$, $6.32(\mathrm{~s}, 1 \mathrm{H}), 6.09(\mathrm{~d}, J=4.9 \mathrm{~Hz}, 1 \mathrm{H}), 2.36(\mathrm{~d}, J=7.0 \mathrm{~Hz}, 2 \mathrm{H}), 1.93$ (sept, $J=7.0 \mathrm{~Hz}$, $1 \mathrm{H}), 0.94(\mathrm{~d}, J=7.0 \mathrm{~Hz}, 6 \mathrm{H}) ;{ }^{13} \mathrm{C} \mathrm{NMR}\left(176 \mathrm{MHz}, \mathrm{CDCl}_{3}\right) \delta 161.6,140.7,139.1$, 130.1, 110.5, 37.6, 27.9, 22.4; HRMS (ESI) m/z: $[\mathrm{M}+\mathrm{H}]^{+}$Calcd for $\mathrm{C}_{9} \mathrm{H}_{13} \mathrm{O}_{3}$ 169.0865; Found 169.0863.<smiles>CCc1cc(O)c(=O)oc1-c1ccccc1</smiles>

\section{5-Ethyl-3-hydroxy-4-isobutyl-6-phenyl-2H-pyran-2-one (22-S4)}

To a solution of 5-ethyl-3-hydroxy-6-phenyl-2H-pyran-2-one (864 mg, $4.0 \mathrm{mmol}, 1$ eq) in dimethylformamide $(2 \mathrm{~mL})$ was added the solution of $N$-bromosuccinimide (783 mg, $4.4 \mathrm{mmol}, 1.1 \mathrm{eq})$ in dimethylformamide $(2 \mathrm{~mL})$ dropwise. The mixture was stirred at $\mathrm{rt}$ for $20 \mathrm{~min}$. The reaction was quenched with brine $(10 \mathrm{~mL})$, and extracted with EtOAc $(5 \times 20 \mathrm{~mL})$. The combined organic layers were washed with brine $(5 \mathrm{x}$ $10 \mathrm{~mL}$ ), dried over $\mathrm{Na}_{2} \mathrm{SO}_{4}$, filtered, and concentrated. The residue was purified by FCC (5:1 hexanes:EtOAc) to yield 4-bromo-5-ethyl-3-hydroxy-6-phenyl-2H-pyran2-one as a yellow solid (1.03 g, 87\%).

Data for 4-bromo-5-ethyl-3-hydroxy-6-phenyl-2H-pyran-2-one: $\mathrm{R}_{\mathrm{f}} 0.46$ (2:1 hexanes: EtOAc); mp: $150-153{ }^{\circ} \mathrm{C}$; IR (thin film) $3295,1682,1371 \mathrm{~cm}^{-1} ;{ }^{1} \mathrm{H}$ NMR (700 MHz, $\left.\mathrm{CDCl}_{3}\right) \delta 7.47-7.45(\mathrm{~m}, 5 \mathrm{H}), 6.65(\mathrm{~s}, 1 \mathrm{H}), 2.59$ (q, $\left.J=7.0 \mathrm{~Hz}, 2 \mathrm{H}\right), 1.22$ (t, $J=7.0$ $\mathrm{Hz}, 3 \mathrm{H}) ;{ }^{13} \mathrm{C} \mathrm{NMR}\left(176 \mathrm{MHz}, \mathrm{CDCl}_{3}\right) \delta 158.6,148.4,139.7,132.2,129.8,128.8$, 128.5, 119.4, 118.2, 24.0, 14.5; HRMS (ESI) m/z: $[\mathrm{M}+\mathrm{H}]^{+}$Calcd for $\mathrm{C}_{13} \mathrm{H}_{12} \mathrm{O}_{3} \mathrm{Br}$ 294.9970; Found 294.9966.

To a thick-walled reaction vessel were added a solution of 4-bromo-5-ethyl-3hydroxy-6-phenyl-2H-pyran-2-one (456 mg, $1.55 \mathrm{mmol}, 1 \mathrm{eq})$ in toluene $(3.1 \mathrm{~mL}$, $0.5 \mathrm{M})$ and $\mathrm{PdCl}_{2}\left(\mathrm{PPh}_{3}\right)_{2}(11 \mathrm{mg}, 0.015 \mathrm{mmol}, 0.01 \mathrm{eq})$. The mixture was stirred for 1 min, and triisobutyl aluminum (1.1 $\mathrm{M}$ in toluene, $1.7 \mathrm{~mL}, 1.85 \mathrm{mmol}, 1.2 \mathrm{eq})$ was added dropwise. The tube was quickly sealed and heated to $100{ }^{\circ} \mathrm{C}$ for $16 \mathrm{~h}$. The reaction was cooled to $\mathrm{rt}$, quenched with $5 \%$ citric acid $(20 \mathrm{~mL})$, and extracted with EtOAc $(5 \times 20 \mathrm{~mL})$. The combined organic layers were dried over $\mathrm{Na}_{2} \mathrm{SO}_{4}$, filtered, and concentrated. The residue was purified by FCC (5:1 hexanes:EtOAc) to yield 22-S4 as a yellow solid (177 mg, $42 \%$ ). 
Data for 22-S4: Rf: 0.40 (4:1 hexanes:EtOAc); mp: $74-78^{\circ} \mathrm{C}$; IR (thin film) 3336, 2959, 1760, $1451 \mathrm{~cm}^{-1} ;{ }^{1} \mathrm{H}$ NMR $\left(700 \mathrm{MHz}, \mathrm{CDCl}_{3}\right) \delta 7.48-7.46(\mathrm{~m}, 2 \mathrm{H}), 7.44-$ $7.42(\mathrm{~m}, 3 \mathrm{H}), 6.05(\mathrm{~s}, 1 \mathrm{H}), 2.49(\mathrm{~d}, J=7.0 \mathrm{~Hz}, 2 \mathrm{H}), 2.46$ (q, $J=7.7 \mathrm{~Hz}, 2 \mathrm{H}), 2.02$ (sept, $J=7.0 \mathrm{~Hz}, 1 \mathrm{H}), 1.07(\mathrm{t}, J=7.7 \mathrm{~Hz}, 3 \mathrm{H}), 1.0(\mathrm{~d}, J=7.0 \mathrm{~Hz}, 6 \mathrm{H}) ;{ }^{13} \mathrm{C} \mathrm{NMR}(176$ $\left.\mathrm{MHz}, \mathrm{CDCl}_{3}\right) \delta 160.9,148.5,138.3,133.1,131.9,129.2,129.0,128.4,120.2,34.7$, 28.5, 22.7, 20.6, 15.4; HRMS (ESI) m/z: $[\mathrm{M}+\mathrm{H}]^{+}$Calcd for $\mathrm{C}_{17} \mathrm{H}_{21} \mathrm{O}_{3}$ 273.1491; Found 273.1502 .<smiles>CCc1cc(O)c(=O)oc1-c1ccccc1</smiles>

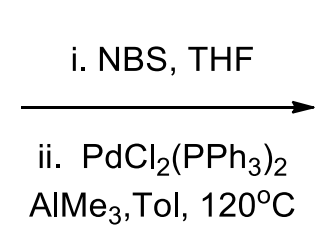<smiles>CCc1c(-c2ccccc2)oc(=O)c(O)c1C</smiles>

\section{5-Ethyl-3-hydroxy-4-methyl-6-phenyl-2H-pyran-2-one (22-S5)}

To a thick-walled reaction vessel were added a solution of 4-bromo-5-ethyl-3hydroxy-6-phenyl-2H-pyran-2-one $(537 \mathrm{mg}, 1.82 \mathrm{mmol}, 1 \mathrm{eq})$ in toluene $(3.64 \mathrm{~mL}$, $0.5 \mathrm{M})$ and $\mathrm{PdCl}_{2}\left(\mathrm{PPh}_{3}\right)_{2}(6.4 \mathrm{mg}, 0.009 \mathrm{mmol}, 0.005 \mathrm{eq})$. The mixture was stirred for $1 \mathrm{~min}$, and trimethyl aluminum $(25 \% \mathrm{w} / \mathrm{w}, 576 \mathrm{mg}, 2.0 \mathrm{mmol}, 1.1 \mathrm{eq})$ was added dropwise. The tube was quickly sealed and heated to $100{ }^{\circ} \mathrm{C}$ for 3 days. The reaction was cooled to rt, quenched with of $5 \%$ citric acid $(50 \mathrm{~mL})$, and extracted with EtOAc $(5 \times 30 \mathrm{~mL})$. The combined organic layers were dried over $\mathrm{Na}_{2} \mathrm{SO}_{4}$, filtered, and concentrated. The residue was purified by FCC (10:1 hexanes:EtOAc) to yield 22-S5 as a white solid (285 mg, $68 \%$ ).

Data for 22-S5: Rf: 0.57 (3:1 hexanes:EtOAc); mp: $144-146{ }^{\circ} \mathrm{C}$; IR (thin film) 3327, 1680, $1643 \mathrm{~cm}^{-1} ;{ }^{1} \mathrm{H}$ NMR $\left(700 \mathrm{MHz}, \mathrm{CDCl}_{3}\right) \delta 7.47-7.42(\mathrm{~m}, 5 \mathrm{H}), 6.11(\mathrm{~s}$, $1 \mathrm{H}), 2.46(\mathrm{q}, J=7.0 \mathrm{~Hz}, 2 \mathrm{H}), 2.21(\mathrm{~s}, 3 \mathrm{H}), 1.15(\mathrm{t}, J=7.0 \mathrm{~Hz}, 3 \mathrm{H}) ;{ }^{13} \mathrm{C}$ NMR $(176$ $\left.\mathrm{MHz}, \mathrm{CDCl}_{3}\right) \delta 160.5,148.4,138.1,133.0,129.3,128.9,128.5,128.4,120.0,21.1$, 14.9, 11.7; HRMS (ESI) $\mathrm{m} / \mathrm{z}$ : $[\mathrm{M}+\mathrm{H}]^{+}$Calcd for $\mathrm{C}_{14} \mathrm{H}_{15} \mathrm{O}_{3} 231.1021$; Found 231.1017 .<smiles>Cc1cc(O)c(=O)oc1-c1ccccc1</smiles>

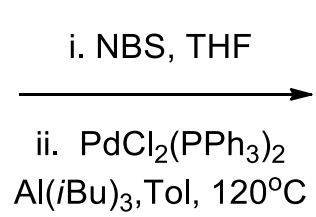<smiles>Cc1c(-c2ccccc2)oc(=O)c(O)c1CC(C)C</smiles>

\section{3-Hydroxy-4-isobutyl-5-methyl-6-phenyl-2H-pyran-2-one (22-S6)}

To a solution of 3-hydroxy-5-methyl-6-phenyl-2H-pyran-2-one (1.36 g, $6.73 \mathrm{mmol}, 1$ eq) in dimethylformamide $(3.5 \mathrm{~mL})$ was added a solution of $N$-bromosuccinimide (1.32 g, $7.4 \mathrm{mmol}, 1.1 \mathrm{eq})$ in dimethylformamide $(3.5 \mathrm{~mL})$ dropwise. The mixture was stirred at $\mathrm{rt}$ for $20 \mathrm{~min}$. The reaction was quenched with brine $(10 \mathrm{~mL})$, and extracted with EtOAc $(5 \times 20 \mathrm{~mL})$. The combined organic layers were washed with brine $(4 \mathrm{x}$ $10 \mathrm{~mL}$ ), dried over $\mathrm{Na}_{2} \mathrm{SO}_{4}$, filtered, and concentrated. The residue was purified by FCC (5:1 hexanes:EtOAc) to yield 4-bromo-3-hydroxy-5-methyl-6-phenyl-2H-pyran- 
2-one as a white solid (1.66 g, 88\%).

Data for 4-bromo-3-hydroxy-5-methyl-6-phenyl-2H-pyran-2-one: $\mathrm{R}_{\mathrm{f}}$ : 0.29 (4:1 hexanes:EtOAc); mp: $190-193{ }^{\circ} \mathrm{C}$; IR (thin film) 3272, 3223, 1682, $1370 \mathrm{~cm}^{-1} ;{ }^{1} \mathrm{H}$ NMR (700 MHz, DMSO-d6) $\delta 10.92(\mathrm{~s}, 1 \mathrm{H}), 7.55-7.49(\mathrm{~m}, 5 \mathrm{H}), 2.15(\mathrm{~s}, 1 \mathrm{H}) ;{ }^{13} \mathrm{C}$ NMR (176 MHz, DMSO-d6) $\delta$ 157.8, 147.0, 140.6, 132.8, 130.0, 129.4, 129.0, 119.9, 113.3, 18.2; HRMS (ESI) m/z: $[\mathrm{M}+\mathrm{H}]^{+}$Calcd for $\mathrm{C}_{12} \mathrm{H}_{10} \mathrm{O}_{3} \mathrm{Br} 280.9813$; Found 280.9812 .

To a thick-walled reaction vessel were added a solution of 4-bromo-3-hydroxy-5methyl-6-phenyl-2H-pyran-2-one (170 mg, $0.6 \mathrm{mmol}, 1 \mathrm{eq})$ in toluene $(1.2 \mathrm{~mL}, 0.5 \mathrm{M})$ and $\mathrm{PdCl}_{2}\left(\mathrm{PPh}_{3}\right)_{2}(4.3 \mathrm{mg}, 0.006 \mathrm{mmol}, 0.01 \mathrm{eq})$. The mixture was stirred for $1 \mathrm{~min}$, and triisobutyl aluminum (1.1 $\mathrm{M}$ in toluene, $0.66 \mathrm{~mL}, 0.73 \mathrm{mmol}, 1.2 \mathrm{eq})$ was added dropwise. The tube was quickly sealed and heated to $100{ }^{\circ} \mathrm{C}$ for $4 \mathrm{~h}$. The reaction was cooled to rt, quenched with of $5 \%$ citric acid $(20 \mathrm{~mL})$, and extracted with EtOAc $(5 \mathrm{x}$ $20 \mathrm{~mL}$ ). The combined organic layers were dried over $\mathrm{Na}_{2} \mathrm{SO}_{4}$, filtered, and concentrated. The residue was purified by FCC (5:1 hexanes:EtOAc) to yield 22-S6 as a white solid (83.5 $\mathrm{mg}, 54 \%)$.

Data for 22-S6: Rf: 0.49 (3:1 hexanes:EtOAc); mp: $142-144{ }^{\circ} \mathrm{C}$; IR (thin film) 3318, 2959, 1683, $1640 \mathrm{~cm}^{-1} ;{ }^{1} \mathrm{H}$ NMR $\left(700 \mathrm{MHz}, \mathrm{CDCl}_{3}\right) \delta 7.49-7.48(\mathrm{~m}, 2 \mathrm{H}), 7.45-$ $7.39(\mathrm{~m}, 3 \mathrm{H}), 6.03(\mathrm{~s}, 1 \mathrm{H}), 2.50(\mathrm{~d}, J=7.0 \mathrm{~Hz}, 2 \mathrm{H}), 2.11$ (s, 3H), 1.99 (sept, $J=7.0$ $\mathrm{Hz}, 1 \mathrm{H}), 1.01(\mathrm{~d}, J=7.0 \mathrm{~Hz}, 6 \mathrm{H}) ;{ }^{13} \mathrm{C} \mathrm{NMR}\left(176 \mathrm{MHz}, \mathrm{CDCl}_{3}\right) \delta 161.0,148.1,137.9$, 133.0, 132.5, 129.2, 128.1, 128.3, 113.8, 35.2, 28.2, 22.7, 14.5; HRMS (ESI) m/z: [M $+\mathrm{H}]^{+}$Calcd for $\mathrm{C}_{16} \mathrm{H}_{19} \mathrm{O}_{3} 259.1334$; Found 259.1335. 


\section{Preparation of Nitroalkene Coupling Partners}

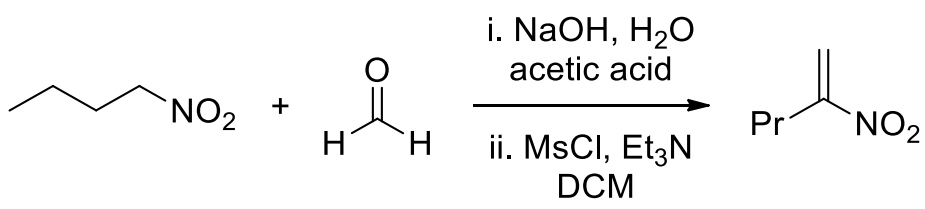

\section{2-Nitropent-1-ene (23-S1)}

To a solution of $\mathrm{NaOH}(210 \mathrm{mg}, 5.25 \mathrm{mmol}, 1.05 \mathrm{eq})$ in water $(2 \mathrm{~mL})$ at $0{ }^{\circ} \mathrm{C}$ was added 1-nitrobutane (515 mg, $5.00 \mathrm{mmol}, 1 \mathrm{eq})$ dropwise. The mixture was stirred at $0{ }^{\circ} \mathrm{C}$ for $10 \mathrm{~min}$, followed by rt for $1 \mathrm{~h}$. The flask was cooled to $0{ }^{\circ} \mathrm{C}$, and formalin $(37 \%$ w/w, $426 \mathrm{mg}, 5.25 \mathrm{mmol}, 1.05 \mathrm{eq})$ was added dropwise. The mixture was stirred at 0 ${ }^{\circ} \mathrm{C}$ for $2 \mathrm{~h}$, followed by an additional $16 \mathrm{~h}$ at $\mathrm{rt}$. The mixture was cooled to $0{ }^{\circ} \mathrm{C}$, and acetic acid (315 mg, $5.5 \mathrm{mmol}, 1.1 \mathrm{eq}$ ) was added dropwise. The mixture was warmed to $\mathrm{rt}$, and extracted with $\mathrm{Et}_{2} \mathrm{O}(5 \times 20 \mathrm{~mL})$. The combined organic layers were washed with brine, dried over $\mathrm{Na}_{2} \mathrm{SO}_{4}$, filtered, and concentrated.

To a solution of the crude material ( $545 \mathrm{mg}, 4.1 \mathrm{mmol}, 1 \mathrm{eq}$ ) from above in $\mathrm{CH}_{2} \mathrm{Cl}_{2}$ $(4.1 \mathrm{~mL}, 1 \mathrm{M})$ at $0{ }^{\circ} \mathrm{C}$ was added $\mathrm{MsCl}(559 \mathrm{mg}, 4.9 \mathrm{mmol}, 1.5 \mathrm{eq})$. The mixture was stirred at $0{ }^{\circ} \mathrm{C}$ for $10 \mathrm{~min}$, upon which time Et $3 \mathrm{~N}$ (1.66 g, $\left.16.4 \mathrm{mmol}, 4 \mathrm{eq}\right)$ was added dropwise. The mixture was stirred at $0{ }^{\circ} \mathrm{C}$ for $1 \mathrm{~h}$, and then warmed to $\mathrm{rt}$ for $1 \mathrm{~h}$. The mixture was quenched with $1 \mathrm{~N} \mathrm{HCl}(30 \mathrm{~mL})$, and extracted with EtOAc $(3 \times 30 \mathrm{~mL})$. The combined organic layers were dried over $\mathrm{Na}_{2} \mathrm{SO}_{4}$, filtered, and concentrated. The residue was purified by FCC (10:1 hexanes:EtOAc) to yield 23-S1 as a yellow oil (113 mg, 24\%).

Data for 23-S1: Rf: 0.54 (5:1 hexanes:EtOAc); IR (thin film) 2966, 2931, 2878, 1722 , 1564, 1528, $1345 \mathrm{~cm}^{-1} ;{ }^{1} \mathrm{H}$ NMR $\left(700 \mathrm{MHz}, \mathrm{CDCl}_{3}\right) \delta 6.42(\mathrm{~d}, J=2.1 \mathrm{~Hz}, 1 \mathrm{H}), 5.53$ $(\mathrm{s}, 1 \mathrm{H}), 2.57$ (dt, $J=7.7,0.7 \mathrm{~Hz}, 2 \mathrm{H}), 1.57$ (sext, $J=7.7 \mathrm{~Hz}, 2 \mathrm{H}), 0.98$ (t, $J=7.7 \mathrm{~Hz}$, $3 \mathrm{H}) ;{ }^{13} \mathrm{C} \mathrm{NMR}\left(176 \mathrm{MHz}, \mathrm{CDCl}_{3}\right) \delta 158.1,117.0,32.0,20.4,13.4$; HRMS (ESI) m/z: $[\mathrm{M}+\mathrm{H}]^{+}$Calcd for $\mathrm{C}_{5} \mathrm{H}_{10} \mathrm{NO}_{2}$ 116.0712; Found 116.0706.

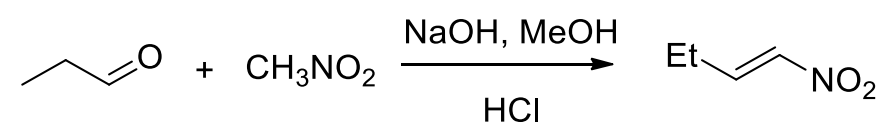

\section{(E)-1-Nitrobut-1-ene (23-S2)}

To a solution of propionaldehyde $(5.80 \mathrm{~g}, 100.0 \mathrm{mmol}, 1 \mathrm{eq})$ in $\mathrm{MeOH}(50 \mathrm{~mL})$ and nitro methane $(24.4 \mathrm{~g}, 400.0 \mathrm{mmol}, 4 \mathrm{eq})$ at $0{ }^{\circ} \mathrm{C}$ was added a solution of $1 \mathrm{~N} \mathrm{NaOH}$ $(250 \mathrm{~mL})$ dropwise. The mixture was stirred at $0{ }^{\circ} \mathrm{C}$ for $2 \mathrm{~h}$. The mixture was then added to a solution of $6 \mathrm{~N} \mathrm{HCl}(60 \mathrm{~mL})$ and stirred at $0{ }^{\circ} \mathrm{C}$ for $15 \mathrm{~min}$. The mixture was warmed to $\mathrm{rt}$, and extracted with EtOAc $(4 \times 60 \mathrm{~mL})$. The combined organic layers were dried over $\mathrm{Na}_{2} \mathrm{SO}_{4}$, filtered and concentrated. The residue was purified by FCC (10:1 hexanes:EtOAc) to yield 23-S2 as an oil (4.36 g, 43\%).

Data for 23-S2: Rf: 0.6 (4:1 hexanes:EtOAc); IR (thin film) 2979, 2942, 2881, 1650 , 
1528, $1356 \mathrm{~cm}^{-1} ;{ }^{1} \mathrm{H} \mathrm{NMR}\left(700 \mathrm{MHz}, \mathrm{CDCl}_{3}\right) \delta 7.33$ (pent, $\left.J=7.0 \mathrm{~Hz}, 1 \mathrm{H}\right), 6.98$ (d, $J$ $=13.3 \mathrm{~Hz}, 1 \mathrm{H}$ ), 2.32 (dpent, $J=7.0,1.4 \mathrm{~Hz}, 2 \mathrm{H}), 1.14(\mathrm{t}, J=7.0 \mathrm{~Hz}, 3 \mathrm{H}) ;{ }^{13} \mathrm{C} \mathrm{NMR}$ $\left(176 \mathrm{MHz}, \mathrm{CDCl}_{3}\right) \delta 144.1,139.2,21.9,11.9 ; \mathrm{HRMS}(\mathrm{ESI}) \mathrm{m} / \mathrm{z}:[\mathrm{M}+\mathrm{H}]^{+}$Calcd for $\mathrm{C}_{4} \mathrm{H}_{8} \mathrm{NO}_{2}$ 102.0555; Found 102.0558.

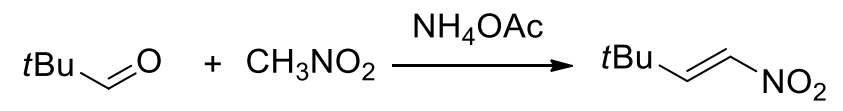

\section{3,3-Dimethyl-1-nitrobut-1-ene (23-S3)}

To a solution of pivaldehyde $(2.15 \mathrm{~g}, 25.0 \mathrm{mmol}, 1 \mathrm{eq})$ in nitro methane $(25 \mathrm{~mL})$ was added ammonium acetate $(962.5 \mathrm{mg}, 12.5 \mathrm{mmol}, 0.5 \mathrm{eq})$. The mixture was heated to reflux for $4.5 \mathrm{~h}$. The mixture was cooled to rt, quenched with brine $(100 \mathrm{~mL})$, and extracted with EtOAc $(4 \times 30 \mathrm{~mL})$. The combined organic layers were dried over $\mathrm{Na}_{2} \mathrm{SO}_{4}$, filtered and concentrated. The residue was purified by FCC (25:1 hexanes:EtOAc) to yield 23-S3 as an oil (1.84 g, 57\%).

Data for 23-S3: Rf: 0.43 (25:1 hexanes:EtOAc); IR (thin film) 2968, 1644, 1530, 1351 $\mathrm{cm}^{-1} ;{ }^{1} \mathrm{H} \mathrm{NMR}\left(700 \mathrm{MHz}, \mathrm{CDCl}_{3}\right) \delta 7.25(\mathrm{td}, J=14.0,1.4 \mathrm{~Hz}, 1 \mathrm{H}), 6.89$ (dd, $J=14.0$, $1.4 \mathrm{~Hz}, 1 \mathrm{H}), 1.15-1.14(\mathrm{~m}, 9 \mathrm{H}) ;{ }^{13} \mathrm{C} \mathrm{NMR}\left(176 \mathrm{MHz}, \mathrm{CDCl}_{3}\right) \delta 152.1,137.2,32.7$, 28.5; HRMS (ESI) m/z: $[\mathrm{M}+\mathrm{H}]^{+}$Calcd for $\mathrm{C}_{6} \mathrm{H}_{12} \mathrm{NO}_{2}$ 130.0868; Found 130.0869.

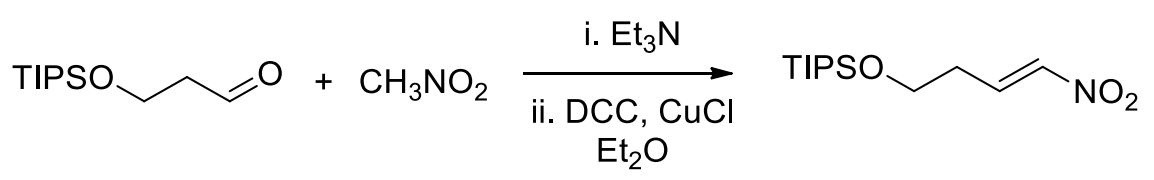

\section{(E)-Triisopropyl((4-nitrobut-3-en-1-yl)oxy)silane (23-S4)}

To a solution of 3-((triisopropylsilyl) oxy)propanal (4.6 g, $20.0 \mathrm{mmol}, 1 \mathrm{eq})$ in 1-nitromethane $(6.1 \mathrm{~g}, 100.0 \mathrm{mmol}, 5 \mathrm{eq})$ was added Et $3 \mathrm{~N}$ (202 mg, $2.0 \mathrm{mmol}, 0.1 \mathrm{eq})$ dropwise. The mixture was stirred at $\mathrm{rt}$ for $20 \mathrm{~h}$. The reaction was quenched with $1 \mathrm{~N}$ $\mathrm{HCl}(20 \mathrm{~mL})$, and extracted with EtOAc $(4 \times 30 \mathrm{~mL})$. The combined organic layers were dried over $\mathrm{Na}_{2} \mathrm{SO}_{4}$, filtered and concentrated.

To a solution of the crude material $(1.4 \mathrm{~g}, 4.8 \mathrm{mmol}, 1 \mathrm{eq})$ from above in $\mathrm{Et}_{2} \mathrm{O}(16 \mathrm{~mL}$, $0.3 \mathrm{M})$ was added $N, N^{\prime}$-dicyclohexylcarbodiimide (1.09 g $\left.5.28 \mathrm{mmol}, 1.1 \mathrm{eq}\right)$ and $\mathrm{CuCl}(143 \mathrm{mg}, 1.44 \mathrm{mmol}, 0.3 \mathrm{eq})$. The mixture was heated to reflux for $17 \mathrm{~h}$. The reaction mixture was cooled to rt, and filtered through Celite with EtOAc. The filtrate was washed with $1 \mathrm{~N} \mathrm{HCl}(2 \times 30 \mathrm{~mL})$, washed with brine $(2 \times 30 \mathrm{~mL})$, dried over $\mathrm{Na}_{2} \mathrm{SO}_{4}$, filtered and concentrated. The residue was purified by FCC (40:1 hexanes:EtOAc) to yield 23-S4 as an oil (484 mg, 37\%, 3:1 E/Z mixture).

Data for 23-S4: Rf: 0.54 (5:1 hexanes:EtOAc); IR (thin film) 2944, 2892, 2867, 1528, 1463, $1352 \mathrm{~cm}^{-1} ;{ }^{1} \mathrm{H}$ NMR $\left(700 \mathrm{MHz}, \mathrm{CDCl}_{3}\right) \delta 7.34$ (pent, $\left.J=7.0 \mathrm{~Hz}, 1 \mathrm{H}\right)(\mathrm{E}), 7.07$ $(\mathrm{d}, J=13.3 \mathrm{~Hz}, 1 \mathrm{H})(\mathrm{E}), 6.99(\mathrm{~d}, J=8.4 \mathrm{~Hz}, 0.35 \mathrm{H})(\mathrm{Z}), 6.38(\mathrm{q}, J=7.0 \mathrm{~Hz}, 0.35 \mathrm{H})$ (Z), $3.88-3.85(\mathrm{~m}, 2.7 \mathrm{H})(\mathrm{E} / \mathrm{Z}), 2.98(\mathrm{q}, J=6.3 \mathrm{~Hz}, 0.7 \mathrm{H})(\mathrm{Z}), 2.49$ (q, $J=6.3 \mathrm{~Hz}$, 2H) (E), $1.14-1.08(\mathrm{~m}, 4 \mathrm{H})(\mathrm{E} / \mathrm{Z}), 1.05$ (d, $J=7.0 \mathrm{~Hz}, 24 \mathrm{H})(\mathrm{E} / \mathrm{Z}) ;{ }^{13} \mathrm{C}$ NMR $(176$ 
$\mathrm{MHz}, \mathrm{CDCl}_{3}$ ) (E) $\delta 140.7,139.9,61.1,32.0,18.0,11.9 ;(\mathrm{Z}) \delta 139.9,138,8,61.3,31.6$, 18.0, 11.9; HRMS (ESI) m/z: [M + Na $]^{+}$Calcd for $\mathrm{C}_{13} \mathrm{H}_{27} \mathrm{NO}_{3} \mathrm{NaSi} 296.1658$; Found 296.1667.<smiles>C/C=C(/CC)[N+](=O)[O-]</smiles>

\section{(E)-3-Nitropent-2-ene (23-S5)}

To a solution of acetaldehyde $(2.64 \mathrm{~g}, 60.0 \mathrm{mmol}, 1 \mathrm{eq})$ in 1-nitropropane (9.35 g, $105.0 \mathrm{mmol}, 1.75 \mathrm{eq})$ at $0{ }^{\circ} \mathrm{C}$ was added $1 \mathrm{~N} \mathrm{KOH}$ in $\mathrm{MeOH}(6 \mathrm{~mL})$ dropwise. The mixture was stirred at $0{ }^{\circ} \mathrm{C}$ for $2.5 \mathrm{~h}$, upon which time a solution of $0.9 \mathrm{~N} \mathrm{H}_{2} \mathrm{SO}_{4}$ in $\mathrm{MeOH}(4 \mathrm{~mL})$ was added. The mixture was stirred at $0{ }^{\circ} \mathrm{C}$ for $10 \mathrm{~min}$, and the solvent was removed under reduced pressure. The residue was dissolved in EtOAc (100 mL), washed sequentially with sat'd aqueous $\mathrm{NaHCO}_{3}(30 \mathrm{~mL})$, water $(30 \mathrm{~mL})$, and brine $(30 \mathrm{~mL})$. The organic layer was dried over $\mathrm{Na}_{2} \mathrm{SO}_{4}$, filtered and concentrated.

To a solution of the crude material ( $3.3 \mathrm{~g}, 24.8 \mathrm{mmol}, 1 \mathrm{eq}$ ) from above in $\mathrm{CH}_{2} \mathrm{Cl}_{2}$ (31 $\mathrm{mL}$ ) was added $\mathrm{Al}_{2} \mathrm{O}_{3}(5.06 \mathrm{~g}, 49.6 \mathrm{mmol}, 2 \mathrm{eq})$. The mixture was heated to $40{ }^{\circ} \mathrm{C}$ for $20 \mathrm{~h}$. The reaction mixture was cooled to rt, and filtered through Celite with $\mathrm{CH}_{2} \mathrm{Cl}_{2}$ to remove solids. The filtrate was concentrated, and distilled (bp $50-60{ }^{\circ} \mathrm{C}, 7$ torr) to yield 23-S5 as an oil (420 mg, $15 \%$ ).

Data for 23-S5: Rf: 0.57 (4:1 hexanes:EtOAc); IR (thin film) 3527, 2980, 1551, 1520 , $1335 \mathrm{~cm}^{-1}$; ${ }^{1} \mathrm{H}$ NMR $\left(700 \mathrm{MHz}, \mathrm{CDCl}_{3}\right) \delta 7.12(\mathrm{q}, J=7.7 \mathrm{~Hz}, 1 \mathrm{H}), 2.60(\mathrm{q}, J=7.7 \mathrm{~Hz}$, $2 \mathrm{H}), 1.87(\mathrm{~d}, J=7.7 \mathrm{~Hz}, 3 \mathrm{H}), 1.09(\mathrm{t}, J=7.7 \mathrm{~Hz}, 3 \mathrm{H}) ;{ }^{13} \mathrm{C} \mathrm{NMR}\left(176 \mathrm{MHz}, \mathrm{CDCl}_{3}\right) \delta$ 153.9, 131.0, 19.6, 13.3, 12.3; HRMS (ESI) m/z: $[\mathrm{M}+\mathrm{H}]^{+}$Calcd for $\mathrm{C}_{5} \mathrm{H}_{10} \mathrm{NO}_{2}$ 116.0712; Found 116.0712.

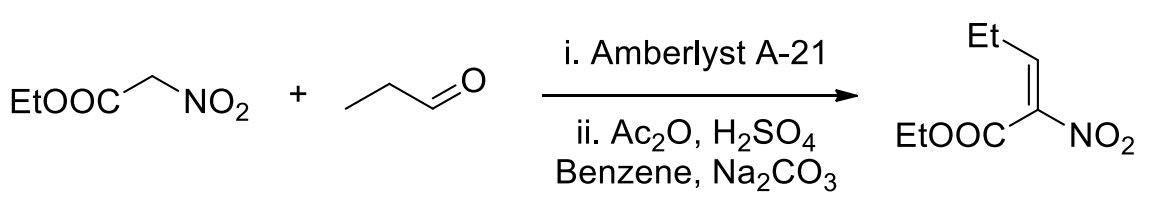

\section{(E)-Ethyl 2-nitropent-2-enoate (23-S6)}

To propinaldehyde $(870 \mathrm{mg}, 15.0 \mathrm{mmol}, 3 \mathrm{eq})$ at $0{ }^{\circ} \mathrm{C}$ was added amberlyst A-21 (250 $\mathrm{mg}$ ). The mixture was stirred at $0{ }^{\circ} \mathrm{C}$ for $5 \mathrm{~min}$, upon which time ethyl nitroacetate (665 mg, $5.0 \mathrm{mmol}, 1 \mathrm{eq}$ ) was added dropwise. The mixture was stirred at $0{ }^{\circ} \mathrm{C}$ for $2 \mathrm{~h}$ and then warmed to rt for 16h. The mixture was filtered through Celite with EtOAc to remove solids. The filtrate was concentrated, and the residue was purified by FCC (5:1 hexanes:EtOAc) to yield an alcohol product as oil $(845 \mathrm{mg}, 88 \%)$.

To a solution of the crude alcohol above $(611 \mathrm{mg}, 3.2 \mathrm{mmol}, 1 \mathrm{eq})$ in acetic anhydride (490 mg, $4.8 \mathrm{mmol}, 1.2 \mathrm{eq}$ ) was added concentrated $\mathrm{H}_{2} \mathrm{SO}_{4}(0.003 \mathrm{~mL})$. The reaction mixture was heated to $60{ }^{\circ} \mathrm{C}$ for $40 \mathrm{~min}$. The mixture was diluted with benzene $(3.2$ 
$\mathrm{mL}$ ), and $\mathrm{Na}_{2} \mathrm{CO}_{3}(339 \mathrm{mg}, 3.2 \mathrm{mmol}, 1 \mathrm{eq}$ ) was added. The mixture was maintained at $60{ }^{\circ} \mathrm{C}$ until the evolution of $\mathrm{CO}_{2}$ gas ceased. The mixture was cooled to $\mathrm{rt}$, and the solvent was removed under reduced pressure. The residue was purified by FCC (20:1 hexanes:EtOAc) to yield 23-S6 as an oil (204 mg, 37\%).

Data for 23-S6: Rf: 0.57 (5:1 hexanes:EtOAc); IR (thin film) 2982, 2942, 1736, 1540 $\mathrm{cm}^{-1} ;{ }^{1} \mathrm{H}$ NMR $\left(700 \mathrm{MHz}, \mathrm{CDCl}_{3}\right) \delta 7.21(\mathrm{t}, J=7.7 \mathrm{~Hz}, 0.35 \mathrm{H})(\mathrm{Z}), 6.84(\mathrm{t}, J=7.7$ $\mathrm{Hz}, 1 \mathrm{H})(\mathrm{E}), 4.34$ (q, $J=7.0 \mathrm{~Hz}, 0.7 \mathrm{H})(\mathrm{Z}), 4.29$ (q, $J=7.0 \mathrm{~Hz}, 2 \mathrm{H})(\mathrm{E}), 2.43$ (pent, $J$ $=7.7 \mathrm{~Hz}, 0.7 \mathrm{H})(\mathrm{Z}), 2.27$ (pent, $J=7.7 \mathrm{~Hz}, 2 \mathrm{H})(\mathrm{E}), 1.33(\mathrm{t}, J=7.0 \mathrm{~Hz}, 1.05 \mathrm{H})(\mathrm{Z})$, $1.30(\mathrm{t}, J=7.0 \mathrm{~Hz}, 3 \mathrm{H})(\mathrm{E}), 1.14(\mathrm{t}, J=7.7 \mathrm{~Hz}, 1.05 \mathrm{H})(\mathrm{Z}), 1.69(\mathrm{t}, J=7.7 \mathrm{~Hz}, 3 \mathrm{H})$ $(\mathrm{E}) ;{ }^{13} \mathrm{C} \mathrm{NMR}\left(176 \mathrm{MHz}, \mathrm{CDCl}_{3}\right)(\mathrm{E}) \delta 158.7,144.5,141.2,62.7,21.6,14.0,12.3$; (Z) $\delta$ 159.8, 144.3, 144.1, 62.6, 21.4, 13.9, 12.5; HRMS (ESI) m/z: $[\mathrm{M}+\mathrm{H}]^{+}$Calcd for $\mathrm{C}_{7} \mathrm{H}_{12} \mathrm{NO}_{4}$ 174.0766; Found 174.0760.

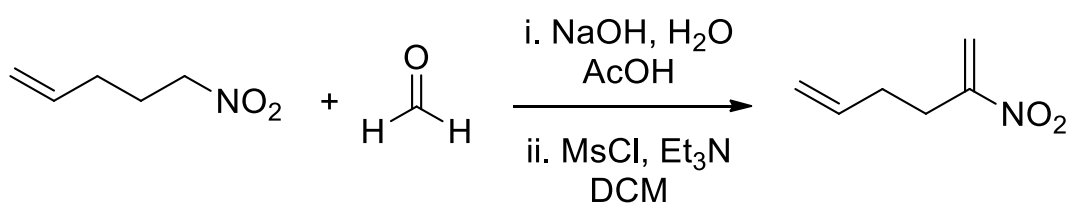

\section{2-Nitrohexa-1,5-diene (23-S7)}

To a solution of $\mathrm{NaOH}(210 \mathrm{mg}, 5.25 \mathrm{mmol}, 1.05 \mathrm{eq})$ in water $(2 \mathrm{~mL})$ at $0{ }^{\circ} \mathrm{C}$ was added 5-nitropent-1-ene (575 mg, $5.00 \mathrm{mmol}, 1 \mathrm{eq})$ dropwise. The mixture was stirred at $0{ }^{\circ} \mathrm{C}$ for $10 \mathrm{~min}$, followed by rt for 1 hour. The mixture was cooled to $0{ }^{\circ} \mathrm{C}$, and formaline $(37 \% \mathrm{w} / \mathrm{w}, 426 \mathrm{mg}, 5.25 \mathrm{mmol}, 1.05 \mathrm{eq})$ was added. The mixture was stirred at $0{ }^{\circ} \mathrm{C}$ for $2 \mathrm{~h}$, followed by an additional $16 \mathrm{~h}$ at $\mathrm{rt}$. The mixture was cooled to $0{ }^{\circ} \mathrm{C}$, and acetic acid (330 mg, $\left.5.50 \mathrm{mmol}, 1.1 \mathrm{eq}\right)$ was added dropwise. The mixture was warmed to $\mathrm{rt}$, and extracted with $\mathrm{Et}_{2} \mathrm{O}(4 \times 30 \mathrm{~mL})$. The combined organic layers were washed with brine, dried over $\mathrm{Na}_{2} \mathrm{SO}_{4}$, filtered and concentrated.

To a solution of the crude material $(290 \mathrm{mg}, 2.0 \mathrm{mmol}, 1 \mathrm{eq})$ from above in $\mathrm{CH}_{2} \mathrm{Cl}_{2}$ $(10 \mathrm{~mL})$ at $0{ }^{\circ} \mathrm{C}$ was added $\mathrm{MsCl}(342 \mathrm{mg}, 3.0 \mathrm{mmol}, 1.5 \mathrm{eq})$. The mixture was stirred at $0{ }^{\circ} \mathrm{C}$ for $10 \mathrm{~min}$, and upon which time $\mathrm{Et} 3 \mathrm{~N}(808 \mathrm{mg}, 8.0 \mathrm{mmol}, 4 \mathrm{eq})$ was added dropwise. The mixture was stirred at $0{ }^{\circ} \mathrm{C}$ for 1 hour, and $\mathrm{rt}$ for 1 hour. The mixture was warmed to $\mathrm{rt}$, quenched with $1 \mathrm{~N} \mathrm{HCl}(20 \mathrm{~mL})$, and extracted with EtOAc $(4 \mathrm{x} 30$ $\mathrm{mL})$. The combined organic layers were dried over $\mathrm{Na}_{2} \mathrm{SO}_{4}$, filtered and concentrated. The residue was purified by FCC (20:1 hexanes:EtOAc) to yield 23-S7 as an oil (128 $\mathrm{mg}, 51 \%)$.

Data for 23-S7: Rf: 0.69 (5:1 hexanes:EtOAc); IR (thin film) 2929, 1714, 1641, 1555, $1526 \mathrm{~cm}^{-1} ;{ }^{1} \mathrm{H} \mathrm{NMR}\left(700 \mathrm{MHz}, \mathrm{CDCl}_{3}\right) \delta 6.46(\mathrm{~s}, 1 \mathrm{H}), 5.79$ (dddd, $J=16.8,11.3,7.0$ $\mathrm{Hz}, 1 \mathrm{H}), 5.56(\mathrm{~s}, 1 \mathrm{H}), 5.09-5.05(\mathrm{~m}, 2 \mathrm{H}), 2.71(\mathrm{t}, J=7.7 \mathrm{~Hz}, 2 \mathrm{H}), 2.32$ (q, $J=7.0$ $\mathrm{Hz}, 2 \mathrm{H}) ;{ }^{13} \mathrm{C} \mathrm{NMR}\left(176 \mathrm{MHz}, \mathrm{CDCl}_{3}\right) \delta 157.3,135.9,117.7,116.5,32.1,29.5$; HRMS (ESI) m/z: [M+ H] $]^{+}$Calcd for $\mathrm{C}_{6} \mathrm{H}_{10} \mathrm{NO}_{2}$ 128.0712; Found 128.0713. 


\section{Syntheses of Substituted Phenols}

General Procedure for Phenol Syntheses:

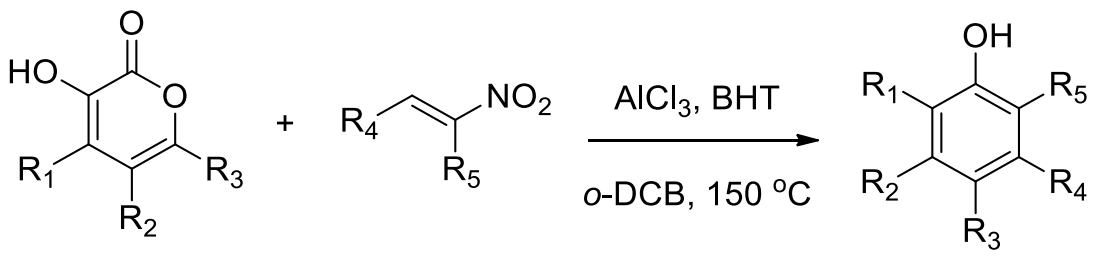

To a thick-walled reaction vessel was added the pyrone, alkene, BHT (0.1 eq) and $\mathrm{AlCl}_{3}(0.1 \mathrm{eq})$. The vessel was flushed with Ar gas for $5 \mathrm{~min}$. 1,2-Dichlorobenzene $(0.5 \mathrm{M})$ was added, and tube was quickly sealed. The reaction mixture was heated to $150^{\circ} \mathrm{C}$ for $16 \mathrm{~h}$ unless otherwise noted. The reaction mixture was cooled to $\mathrm{rt}$, and the mixture was directly purified by FCC without aqueous work up.<smiles>CPc1ccccc1O</smiles>

\section{2-Propylphenol (25)}

3-Hydroxy-2H-pyran-2-one ( $89.6 \mathrm{mg}, 0.8 \mathrm{mmol}, 4 \mathrm{eq}$ ) and 2-nitropent-1-ene (23 mg, $0.2 \mathrm{mmol}, 1 \mathrm{eq})$ were subjected to the general procedure for $1 \mathrm{~h}$. Purification by FCC (20:1 hexanes:EtOAc) yielded 25 as a yellow oil (22 $\mathrm{mg}, 81 \%$ ). Spectroscopic data for $\mathbf{2 5}$ matched those previously reported. ${ }^{2}$<smiles>CCc1cccc(O)c1</smiles>

\section{3-Ethylphenol (26)}

3-Hydroxy-2H-pyran-2-one ( $89.6 \mathrm{mg}, 0.8 \mathrm{mmol}, 4 \mathrm{eq})$ and 1-nitrobut-1-ene (20.2 mg, $0.2 \mathrm{mmol}, 1 \mathrm{eq})$ were subjected to the general procedure. Purification by FCC (10:1 hexanes:EtOAc) yielded 26 as a colorless oil (19.8 mg, 81\%). Spectroscopic data for 26 matched those previously reported. ${ }^{3}$<smiles>CCc1ccccc1O</smiles>

\section{2-Ethylphenol (27)}

3-Hydroxy-2H-pyran-2-one (89.6 mg, $0.8 \mathrm{mmol}, 4 \mathrm{eq}$ ) and 2-nitrobut-1-ene (20.2 $\mathrm{mg}$, $0.2 \mathrm{mmol}, 1 \mathrm{eq})$ were subjected to the general procedure for $3 \mathrm{~h}$. Purification by FCC (15:1 hexanes:EtOAc) yielded 27 as a yellow oil (17.9 mg, 73\%). Spectroscopic data for 27 matched those previously reported. ${ }^{4}$ 
<smiles>Oc1cccc(Br)c1</smiles>

\section{3-Benzylphenol (28)}

3-Hydroxy-2H-pyran-2-one ( $89.6 \mathrm{mg}, 0.8 \mathrm{mmol}, 4 \mathrm{eq}$ ) and (3-nitroallyl)benzene (32.6 $\mathrm{mg}, 0.2 \mathrm{mmol}, 1 \mathrm{eq})$ were subjected to the general procedure. Purification by FCC (10:1 hexanes:EtOAc) yielded 28 as a colorless oil (23.2 $\mathrm{mg}, 63 \%)$. Spectroscopic data for $\mathbf{2 8}$ matched those previously reported. ${ }^{5}$<smiles>Oc1ccccc1Br</smiles>

\section{2-Benzylphenol (29)}

3-Hydroxy-2H-pyran-2-one ( $89.6 \mathrm{mg}, 0.8 \mathrm{mmol}, 4 \mathrm{eq}$ ) and (2-nitroallyl)benzene (32.6 $\mathrm{mg}, 0.2 \mathrm{mmol}, 1 \mathrm{eq}$ ) were subjected to the general procedure for $2 \mathrm{~h}$. Purification by FCC (10:1 hexanes:EtOAc) yielded 29 as a yellow solid (27.5 mg, 74\%). Spectroscopic data for $\mathbf{2 9}$ matched those previously reported. ${ }^{6}$<smiles>CC(C)(C)c1cccc(O)c1</smiles>

\section{3-(Tert-butyl)phenol (30)}

3-Hydroxy-2H-pyran-2-one (89.6 mg, $0.8 \mathrm{mmol}, 4 \mathrm{eq})$ and 3,3-dimethyl-1-nitrobut -1-ene $(25.8 \mathrm{mg}, 0.2 \mathrm{mmol}, 1 \mathrm{eq})$ were subjected to the general procedure at $180{ }^{\circ} \mathrm{C}$. Purification by FCC (10:1 hexanes:EtOAc) yielded $\mathbf{3 0}$ as a colorless oil (25.5 mg, $85 \%$ ). Spectroscopic data for $\mathbf{3 0}$ matched those previously reported. ${ }^{7}$<smiles>Oc1cccc(CC[OH+][S-])c1</smiles>

\section{3-(2-((Triisopropylsilyl)oxy)ethyl)phenol (31).}

3-Hydroxy-2H-pyran-2-one (67.2 $\mathrm{mg}, 0.6 \mathrm{mmol}, 3 \mathrm{eq})$ and triisopropyl((4-nitrobut-3en-1-yl)oxy)silane (54.6 mg, $0.2 \mathrm{mmol}, 1 \mathrm{eq}$ ) were subjected to the general procedure. Purification by FCC (20:1 hexanes:EtOAc) yielded $\mathbf{3 1}$ as a yellow oil $(32.5 \mathrm{mg}, 55 \%)$.

Data for 31: $\mathrm{R}_{\mathrm{f}}$ 0.49 (5:1 hexanes:EtOAc); IR (thin film) 3344, 2943, 2892, 2866, 1589, $1458 \mathrm{~cm}^{-1} ;{ }^{1} \mathrm{H}$ NMR $\left(700 \mathrm{MHz}, \mathrm{CDCl}_{3}\right) \delta 7.15(\mathrm{t}, J=7.7 \mathrm{~Hz}, 1 \mathrm{H}), 6.80(\mathrm{~d}, J=$ $7.7 \mathrm{~Hz}, 1 \mathrm{H}), 6.71(\mathrm{~s}, 1 \mathrm{H}), 6.80(\mathrm{~d}, J=7.7 \mathrm{~Hz}, 1 \mathrm{H}), 4.89(\mathrm{~s}, 1 \mathrm{H}), 3.87(\mathrm{t}, J=7.7 \mathrm{~Hz}$, $2 \mathrm{H}), 2.82(\mathrm{t}, J=7.7 \mathrm{~Hz}, 2 \mathrm{H}), 1.11-1.07(\mathrm{~m}, 3 \mathrm{H}), 1.05(\mathrm{~d}, J=7.0 \mathrm{~Hz}, 18 \mathrm{H}) ;{ }^{13} \mathrm{C}$ NMR $\left(176 \mathrm{MHz}, \mathrm{CDCl}_{3}\right) \delta 155.4,141.1,129.4,121.7,116.1,113.0,64.8,39.6,18.0$, 
12.0; HRMS (ESI) m/z: $\left[\mathrm{M}+\mathrm{Na}^{+}\right.$Calcd for $\mathrm{C}_{17} \mathrm{H}_{30} \mathrm{O}_{2} \mathrm{SiNa}$ 317.1913; Found 317.1909 .<smiles>C=CCCc1ccccc1O</smiles>

\section{2-(But-3-en-1-yl)phenol (32)}

3-Hydroxy-2H-pyran-2-one (67.2 $\mathrm{mg}, 0.6 \mathrm{mmol}, 3 \mathrm{eq})$ and 2-nitrohexa-1,5-diene (25.4 mg, $0.2 \mathrm{mmol}, 1 \mathrm{eq})$ were subjected to the general procedure for $1 \mathrm{~h}$. Purification by FCC (40:1 hexanes:EtOAc) yielded 32 as a colorless oil (18 mg, 61\%). Spectroscopic data for $\mathbf{3 2}$ matched those previously reported. ${ }^{8}$<smiles>CCOC(=O)c1cccc(O)c1</smiles>

\section{Ethyl 3-hydroxybenzoate (33)}

3-Hydroxy-2H-pyran-2-one (89.6 mg, $0.8 \mathrm{mmol}, 4 \mathrm{eq})$ and ethyl 3-nitroacrylate (29.0 $\mathrm{mg}, 0.2 \mathrm{mmol}, 1 \mathrm{eq})$ were subjected to the general procedure for $10 \mathrm{~min}$. Purification by FCC (20:1 hexanes:EtOAc) yielded $\mathbf{3 3}$ as a white solid (14.6 mg of 33, $7.1 \mathrm{mg}$ of the corresponding regioisomer, $\mathrm{rr}=2: 1,65 \%$ yield). Spectroscopic data for $\mathbf{3 3}$ matched those previously reported. ${ }^{9}$<smiles>CCc1cccc(O)c1C</smiles>

\section{3-Ethyl-2-methylphenol (34)}

3-Hydroxy-2H-pyran-2-one ( $89.6 \mathrm{mg}, 0.8 \mathrm{mmol}, 4 \mathrm{eq}$ ) and ethyl 3-nitroacrylate (23.0 $\mathrm{mg}, 0.2 \mathrm{mmol}, 1 \mathrm{eq})$ were subjected to the general procedure. Purification by FCC (10:1 hexanes:EtOAc) yielded 34 as a yellow solid (19.0 mg, 70\%). Spectroscopic data for 34 matched those previously reported. ${ }^{10}$<smiles>CCc1c(C)cccc1O</smiles>

\section{2-Ethyl-3-methylphenol (35).}

3-Hydroxy-2H-pyran-2-one ( $89.6 \mathrm{mg}, 0.8 \mathrm{mmol}, 4 \mathrm{eq})$ and 3-nitropent-2-ene (23 mg, $0.2 \mathrm{mmol}, 1 \mathrm{eq})$ were subjected to the general procedure. Purification by FCC (20:1 hexaness:EtOAc) yielded 35 as a yellow oil (30.2 $\mathrm{mg}, 55 \%)$.

Data for 35: Rf: 0.48 (5:1 hexanes:EtOAc); IR (thin film) 3387, 2967, 1584, 1465 
$\mathrm{cm}^{-1} ;{ }^{1} \mathrm{H}$ NMR $\left(700 \mathrm{MHz}, \mathrm{CDCl}_{3}\right) \delta 6.96(\mathrm{t}, J=7.7 \mathrm{~Hz}, 1 \mathrm{H}), 6.75(\mathrm{~d}, J=7.7 \mathrm{~Hz}, 1$ $\mathrm{H}), 6.62(\mathrm{~d}, J=7.7 \mathrm{~Hz}, 1 \mathrm{H}), 4.61(\mathrm{~s}, 1 \mathrm{H}), 2.66(\mathrm{q}, J=7.7 \mathrm{~Hz}, 2 \mathrm{H}), 2.31(\mathrm{~s}, 3 \mathrm{H})$, $1.15(\mathrm{t}, J=7.7 \mathrm{~Hz}, 3 \mathrm{H}) ;{ }^{13} \mathrm{C} \mathrm{NMR}\left(176 \mathrm{MHz}, \mathrm{CDCl}_{3}\right) \delta 153.3,137.6,128.7,126.2$, 122.8, 112.9, 19.5, 19.3, 13.4; HRMS (ESI) m/z: $[\mathrm{M}+\mathrm{H}]^{+}$Calcd for $\mathrm{C}_{9} \mathrm{H}_{13} \mathrm{O}$ 137.0966; Found 137.0972.<smiles>Cc1c(O)cccc1-c1ccccc1</smiles>

\section{2-Methyl-[1,1'-biphenyl]-3-ol (36)}

3-Hydroxy-2H-pyran-2-one (112 mg, $1 \mathrm{mmol}, 5 \mathrm{eq})$ and (2-nitroprop-1-en-1-yl) benzene $(32.6 \mathrm{mg}, 0.2 \mathrm{mmol}, 1 \mathrm{eq})$ were subjected to the general procedure at $160{ }^{\circ} \mathrm{C}$ for 2 days. Purification by FCC (10:1 hexanes:EtOAc) yielded 36 as a yellow oil (26.8 mg, 73\%). Spectroscopic data for $\mathbf{3 6}$ matched those previously reported. ${ }^{11}$<smiles>CCOC(=O)c1cccc(O)c1C</smiles>

\section{Ethyl 3-hydroxy-2-methylbenzoate (37)}

3-Hydroxy-2H-pyran-2-one (89.6 mg, $0.8 \mathrm{mmol}, 4 \mathrm{eq}$ ) and ethyl 3-nitrobut-2-enoate $(31.8 \mathrm{mg}, 0.2 \mathrm{mmol}, 1 \mathrm{eq})$ were subjected to the general procedure at $160{ }^{\circ} \mathrm{C}$. Purification by FCC (10:1 hexanes:EtOAc) yielded 37 as a yellow solid (20.9 mg, $58 \%$ ). Spectroscopic data for 37 matched those previously reported. ${ }^{12}$<smiles>CCOC(=O)c1cccc(O)c1CC</smiles>

\section{Ethyl 2-ethyl-3-hydroxybenzoate (38).}

3-Hydroxy-2H-pyran-2-one (67.2 $\mathrm{mg}, 0.6 \mathrm{mmol}, 3 \mathrm{eq}$ ) and ethyl 3-nitropent-2-enoate (34.6 $\mathrm{mg}, 0.2 \mathrm{mmol}, 1 \mathrm{eq}$ ) were subjected to the general procedure. Purification by FCC (20:1 hexanes:EtOAc) yielded $\mathbf{3 8}$ as a yellow solid (15.7 $\mathrm{mg}$ of $\mathbf{3 8}, 8.4 \mathrm{mg}$ of $\mathbf{3 9}$ $\mathrm{rr}=2: 1,63 \%$ yield).

Data for 38: Rf: 0.27 (5:1 hexanes:EtOAc); mp: $67-69^{\circ} \mathrm{C}$. IR (thin film) 3406, 2978, 2936, 1693, 1585, 1463, $1287 \mathrm{~cm}^{-1} ;{ }^{1} \mathrm{H} \mathrm{NMR}\left(700 \mathrm{MHz}, \mathrm{CDCl}_{3}\right) \delta 7.37$ (d, $J=7.7 \mathrm{~Hz}$, $1 \mathrm{H}), 7.10(\mathrm{t}, J=7.7 \mathrm{~Hz}, 1 \mathrm{H}), 6.92(\mathrm{~d}, J=7.7 \mathrm{~Hz}, 1 \mathrm{H}), 5.26(\mathrm{~s}, 1 \mathrm{H}), 4.36(\mathrm{q}, J=7.0$ $\mathrm{Hz}, 2 \mathrm{H}), 2.92(\mathrm{q}, J=7.7 \mathrm{~Hz}, 2 \mathrm{H}), 1.39$ (t, $J=7.0 \mathrm{~Hz}, 3 \mathrm{H}), 1.23(\mathrm{t}, J=7.7 \mathrm{~Hz}, 3 \mathrm{H})$; ${ }^{13} \mathrm{C} \mathrm{NMR}\left(176 \mathrm{MHz}, \mathrm{CDCl}_{3}\right) \delta 168.3,154.0,132.0,131.2,126.4,122.5,118.5,61.1$, 20.3, 14.3, 14.3; HRMS (EI) $\mathrm{m} / \mathrm{z}$ : $[\mathrm{M}]^{+}$Calcd for $\mathrm{C}_{11} \mathrm{H}_{14} \mathrm{O}_{3}$ 194.0943; Found 194.0938 . 
<smiles>CCOC(=O)c1c(O)cccc1CC</smiles>

\section{Ethyl 2-ethyl-6-hydroxybenzoate (39)}

3-Hydroxy-2H-pyran-2-one ( $89.6 \mathrm{mg}, 0.8 \mathrm{mmol}, 4 \mathrm{eq})$ and ethyl 3-nitrobut-2-enoate (34.6 $\mathrm{mg}, 0.2 \mathrm{mmol}, 1 \mathrm{eq}$ ) were subjected to the general procedure. Purification by FCC (100:1 hexanes:EtOAc) yielded 39 as a colorless oil (6.3 $\mathrm{mg}, 16 \%)$. Spectroscopic data for $\mathbf{3 9}$ matched those previously reported. ${ }^{13}$<smiles>CCc1cccc(CC(C)C)c1O</smiles>

\section{2-Ethyl-6-isobutylphenol (40).}

3-Hydroxy-4-isobutyl-2H-pyran-2-one $(67.2 \mathrm{mg}, 0.4 \mathrm{mmol}, 2 \mathrm{eq})$ and 2-nitrobut-1ene $(20.2 \mathrm{mg}, 0.2 \mathrm{mmol}, 1 \mathrm{eq})$ were subjected to the general procedure for $15 \mathrm{~min}$. Purification by FCC (40:1 hexanes:EtOAc) yielded 40 as a yellow oil (23.6 mg, 66\%).

Data for 40: Rf: 0.57 (7:1 hexanes:EtOAc); IR (thin film) 3580, 2958, 2931, 2869, 1457, $1188 \mathrm{~cm}^{-1} ;{ }^{1} \mathrm{H}$ NMR $\left(700 \mathrm{MHz}, \mathrm{CDCl}_{3}\right) \delta 7.01(\mathrm{dd}, J=7.0,1.4 \mathrm{~Hz}, 1 \mathrm{H}), 6.95$ $(\mathrm{dd}, J=7.0,1.4 \mathrm{~Hz}, 1 \mathrm{H}), 6.82(\mathrm{t}, J=7.0 \mathrm{~Hz}, 1 \mathrm{H}), 4.63(\mathrm{~s}, 1 \mathrm{H}), 2.64(\mathrm{q}, J=7.7 \mathrm{~Hz}$, $2 \mathrm{H}), 2.48(\mathrm{~d}, J=7.7 \mathrm{~Hz}, 2 \mathrm{H}), 1.93$ (sept, $J=7.0 \mathrm{~Hz}, 1 \mathrm{H}), 1.25$ (t, $J=7.7 \mathrm{~Hz}, 3 \mathrm{H}$ ), $0.95(\mathrm{~d}, J=7.0 \mathrm{~Hz}, 6 \mathrm{H}) ;{ }^{13} \mathrm{C} \mathrm{NMR}\left(176 \mathrm{MHz}, \mathrm{CDCl}_{3}\right) \delta 151.5,129.3,128.8,126.8$, 126.7, 120.2, 39.5, 28.9, 23.1, 22.7, 14.0; HRMS (EI) m/z: $[\mathrm{M}]^{+}$Calcd for $\mathrm{C}_{12} \mathrm{H}_{18} \mathrm{O}$ 178.1358; Found 178.1353.<smiles>CC(C)Cc1ccc(Br)cc1O</smiles>

\section{5-Benzyl-2-isobutylphenol (41).}

3-Hydroxy-4-isobutyl-2H-pyran-2-one (67.2 mg, $0.4 \mathrm{mmol}, 2 \mathrm{eq})$ and (3-nitroallyl) benzene $(27.2 \mathrm{mg}, 0.2 \mathrm{mmol}, 1 \mathrm{eq})$ were subjected to the general procedure for $4 \mathrm{~h}$. Purification by FCC (40:1 hexanes:EtOAc) yielded 41 as a yellow oil (24.4 mg, 51\%).

Data for 41: Rf: 0.15 (5:1 hexanes: $\mathrm{CH}_{2} \mathrm{Cl}_{2}$ ); IR (thin film) 3534, 3431, 3027, 2954, 2925, 2867, $1425 \mathrm{~cm}^{-1} ;{ }^{1} \mathrm{H} \mathrm{NMR}\left(700 \mathrm{MHz}, \mathrm{CDCl}_{3}\right) \delta 7.30$ (t, $\left.J=7.7 \mathrm{~Hz}, 2 \mathrm{H}\right), 7.23$ $7.20(\mathrm{~m}, 3 \mathrm{H}), 7.00(\mathrm{~d}, J=7.7 \mathrm{~Hz}, 1 \mathrm{H}), 6.73(\mathrm{dd}, J=7.7,1.4 \mathrm{~Hz}, 1 \mathrm{H}), 6.57(\mathrm{~d}, J=$ $1.4 \mathrm{~Hz}, 1 \mathrm{H}), 4.58$ (s, $1 \mathrm{H}), 3.92$ (s, $2 \mathrm{H}), 2.45$ (d, $J=7.0 \mathrm{~Hz}, 2 \mathrm{H}), 1.92$ (sept, $J=7.0$ $\mathrm{Hz}, 1 \mathrm{H}), 0.94(\mathrm{~d}, J=7.0 \mathrm{~Hz}, 6 \mathrm{H}) ;{ }^{13} \mathrm{C} \mathrm{NMR}\left(176 \mathrm{MHz}, \mathrm{CDCl}_{3}\right) \delta 153.6,141.1$, 140.4, 131.2, 129.0, 128.5, 126.1, 125.1, 121.1, 115.8, 41.5, 39.0, 28.9, 22.6; HRMS (EI) $\mathrm{m} / \mathrm{z}:[\mathrm{M}]^{+}$Calcd for $\mathrm{C}_{17} \mathrm{H}_{20} \mathrm{O} 240.1514$; Found 240.1513 . 
<smiles>CCc1ccc(C)c(O)c1</smiles>

\section{2-Methyl-5-ethylphenol (42)}

3-Hydroxy-4-methyl-2H-pyran-2-one (75.6 mg, $0.6 \mathrm{mmol}, 3 \mathrm{eq}$ ) and 1-nitrobut-1-ene (20.2 $\mathrm{mg}, 0.2 \mathrm{mmol}, 1 \mathrm{eq})$ were subjected to the general procedure for $3 \mathrm{~h}$. Purification by FCC (15:1 hexanes:EtOAc) yielded $\mathbf{4 2}$ as a yellow oil $(22.3 \mathrm{mg}, 82 \%)$. Spectroscopic data for $\mathbf{4 2}$ matched those previously reported. ${ }^{14}$<smiles>CCc1cccc(C)c1O</smiles>

\section{2-Ethyl-6-methylphenol (43)}

3-Hydroxy-4-methyl-2H-pyran-2-one (50.4 mg, $0.4 \mathrm{mmol}, 2 \mathrm{eq}$ ) and 2-nitrobut-1-ene (20.2 $\mathrm{mg}, 0.2 \mathrm{mmol}, 1 \mathrm{eq}$ ) were subjected to the general procedure for $15 \mathrm{~min}$. Purification by FCC (30:1 hexanes:EtOAc) yielded 43 as a yellow oil (19.3 mg, 71\%). Spectroscopic data for $\mathbf{4 3}$ matched those previously reported. ${ }^{15}$<smiles>Cc1ccc(Br)cc1O</smiles>

\section{5-Benzyl-2-methylphenol (44).}

3-Hydroxy-4-methyl-2H-pyran-2-one (50.4 mg, $0.4 \mathrm{mmol}, 2 \mathrm{eq})$ and (3-nitroallyl) benzene (32.6 mg, $0.2 \mathrm{mmol}, 1 \mathrm{eq})$ were subjected to the general procedure. Purification by FCC (40:1 hexanes:EtOAc) yielded 44 as a yellow solid (26.6 mg, $67 \%)$.

Data for 44: Rf: 0.48 (7:1 hexanes: EtOAc); mp: $34-35^{\circ} \mathrm{C}$; IR (thin film) 3565, 3027, 2957, 2922, 1594, 1470, $1199 \mathrm{~cm}^{-1} ;{ }^{1} \mathrm{H}$ NMR $\left(700 \mathrm{MHz}, \mathrm{CDCl}_{3}\right) \delta 7.31(\mathrm{t}, J=7.7 \mathrm{~Hz}$, $2 \mathrm{H}), 7.23-7.20(\mathrm{~m}, 3 \mathrm{H}), 7.06(\mathrm{~d}, J=7.7 \mathrm{~Hz}, 1 \mathrm{H}), 6.73(\mathrm{~d}, J=7.7 \mathrm{~Hz}, 1 \mathrm{H}), 6.58(\mathrm{~s}$, $1 \mathrm{H}), 4.65(\mathrm{~d}, J=2.8 \mathrm{~Hz}, 1 \mathrm{H}), 3.92(\mathrm{~s}, 2 \mathrm{H}), 2.23(\mathrm{~s}, 3 \mathrm{H}) ;{ }^{13} \mathrm{C} \mathrm{NMR}(176 \mathrm{MHz}$, $\left.\mathrm{CDCl}_{3}\right) \delta 153.8,141.1,140.5,131.0,129.0,128.5,126.1,121.4,121.3,115.5,41.5$, 15.4; HRMS (ESI) m/z: [M + H $]^{+}$Calcd for $\mathrm{C}_{14} \mathrm{H}_{15} \mathrm{O}$ 199.1123; Found 199.1116.<smiles>Cc1cccc(Br)c1O</smiles>

\section{2-Benzyl-6-methylphenol (45)}

3-Hydroxy-4-methyl-2H-pyran-2-one (50.4 mg, $0.4 \mathrm{mmol}, 2 \mathrm{eq}$ ) and (2-nitroallyl) benzene $(32.6 \mathrm{mg}, 0.2 \mathrm{mmol}, 1 \mathrm{eq})$ were subjected to the general procedure for 45 
min. Purification by FCC (20:1 hexanes:EtOAc) yielded $\mathbf{4 5}$ as a white solid (31.7 mg, $80 \%$ ). Spectroscopic data for $\mathbf{4 5}$ matched those previously reported. ${ }^{16}$<smiles>CCCc1ccc(C)c(O)c1</smiles>

\section{2-Methyl-5-propylphenol (46).}

3-Hydroxy-4-methyl-2H-pyran-2-one (50.4 mg, $0.4 \mathrm{mmol}, 2 \mathrm{eq})$ and 1-nitropent-1 -ene (23 mg, $0.2 \mathrm{mmol}, 1 \mathrm{eq})$ were subjected to the general procedure at $180{ }^{\circ} \mathrm{C}$ for 1 hour. Purification by FCC (20:1 hexanes:EtOAc) yielded 46 as a yellow oil (23.2 mg, $77 \%)$.

Data for 46: Rf: 0.45 (5:1 hexanes: EtOAc); IR (thin film) 3392, 2959, 2929, 2871, 1587, 1456, 1420, $1244 \mathrm{~cm}^{-1}$; ${ }^{1} \mathrm{H}$ NMR $\left(700 \mathrm{MHz}, \mathrm{CDCl}_{3}\right) \delta 7.03(\mathrm{~d}, J=7.7 \mathrm{~Hz}, 1 \mathrm{H})$, $6.69(\mathrm{dd}, J=7.7,1.4 \mathrm{~Hz}, 1 \mathrm{H}), 6.62(\mathrm{~d}, J=1.4 \mathrm{~Hz}, 1 \mathrm{H}), 4.69(\mathrm{~s}, 1 \mathrm{H}), 2.52(\mathrm{t}, J=7.7$ $\mathrm{Hz}, 2 \mathrm{H}), 2.23(\mathrm{~s}, 3 \mathrm{H}), 1.63(\mathrm{sext}, J=7.7 \mathrm{~Hz}, 2 \mathrm{H}), 0.95(\mathrm{t}, J=7.7 \mathrm{~Hz}, 3 \mathrm{H}) ;{ }^{13} \mathrm{C}$ NMR (176 MHz, $\left.\mathrm{CDCl}_{3}\right) \delta 153.6,142.1,130.8,120.9,120.7,115.0,37.6,24.5,15.4$, 13.9; HRMS (EI) m/z: [M] $]^{+}$Calcd for $\mathrm{C}_{10} \mathrm{H}_{14} \mathrm{O}$ 150.1045; Found 150.1051.<smiles>C=CCCc1ccc(C)c(O)c1</smiles>

\section{5-(But-3-en-1-yl)-2-methylphenol (47).}

3-Hydroxy-4-methyl-2H-pyran-2-one (50.4 mg, $0.4 \mathrm{mmol}, 2$ eq) and 1-nitrohexa-1,5diene $(25.4 \mathrm{mg}, 0.2 \mathrm{mmol}, 1 \mathrm{eq})$ were subjected to the general procedure for $6 \mathrm{~h}$. Purification by FCC (20:1 hexanes:EtOAc) yielded 47 as a yellow oil (19.8 mg, 61\%).

Data for 47: Rf: 0.43 (5:1 hexanes:EtOAc); IR (thin film) 3400, 2977, 2925, 2856, 1640, 1587, $1419 \mathrm{~cm}^{-1} ;{ }^{1} \mathrm{H}$ NMR $\left(700 \mathrm{MHz}, \mathrm{CDCl}_{3}\right) \delta 7.03(\mathrm{~d}, J=7.0 \mathrm{~Hz}, 1 \mathrm{H}), 6.69$ $(\mathrm{d}, J=7.0 \mathrm{~Hz}, 1 \mathrm{H}), 6.63(\mathrm{~s}, 1 \mathrm{H}), 5.86$ (ddt, $J=16.8,10.5,6.3 \mathrm{~Hz}, 1 \mathrm{H}), 5.47$ (d, $J=$ $17.5 \mathrm{~Hz}, 1 \mathrm{H}), 4.98(\mathrm{~d}, J=10.5 \mathrm{~Hz}, 1 \mathrm{H}), 4.66(\mathrm{~s}, 1 \mathrm{H}), 2.64(\mathrm{t}, J=7.7 \mathrm{~Hz}, 2 \mathrm{H}), 2.35$ $(\mathrm{q}, J=7.7 \mathrm{~Hz}, 2 \mathrm{H}), 2.22(\mathrm{~s}, 3 \mathrm{H}) ;{ }^{13} \mathrm{C} \mathrm{NMR}\left(176 \mathrm{MHz}, \mathrm{CDCl}_{3}\right) \delta 153.6,141.2,138.1$, 130.8, 120.9, 120.8, 115.0, 114.9, 35.5, 34.9, 15.4; HRMS (EI) m/z: [M] ${ }^{+}$Calcd for $\mathrm{C}_{11} \mathrm{H}_{14} \mathrm{O}$ 162.1045; Found 162.1042.<smiles>C=CCCc1cccc(C)c1O</smiles>

\section{2-(But-3-en-1-yl)-6-methylphenol (48).}

3-Hydroxy-4-methyl-2H-pyran-2-one (50.4 mg, $0.4 \mathrm{mmol}, 2 \mathrm{eq}$ ) and 2-nitrohexa-1,5diene $(25.4 \mathrm{mg}, 0.2 \mathrm{mmol}, 1 \mathrm{eq})$ were subjected to the general procedure for $15 \mathrm{~min}$. 
Purification by FCC (50:1 hexanes:EtOAc) yielded 48 as a colorless oil (17.1 mg, $53 \%)$.

Data for 48: Rf: 0.57 (5:1 hexanes:EtOAc); IR (thin film) 3568, 2923, 2854, 1640, 1594, $1468 \mathrm{~cm}^{-1} ;{ }^{1} \mathrm{H}$ NMR $\left(700 \mathrm{MHz}, \mathrm{CDCl}_{3}\right) \delta 6.99(\mathrm{~d}, J=7.7 \mathrm{~Hz}, 2 \mathrm{H}), 6.79(\mathrm{t}, J=$ $7.7 \mathrm{~Hz}, 1 \mathrm{H}$ ), 5.91 (ddt, $J=16.8,13.3,7.0 \mathrm{~Hz}, 1 \mathrm{H}), 5.09$ (dd, $J=17.5,1.4 \mathrm{~Hz}, 1 \mathrm{H})$, $5.05(\mathrm{~d}, J=10.5 \mathrm{~Hz}, 1 \mathrm{H}), 4.63(\mathrm{~s}, 1 \mathrm{H}), 2.71(\mathrm{t}, J=7.7 \mathrm{~Hz}, 2 \mathrm{H}), 2.38$ (q, $J=7.7 \mathrm{~Hz}$, $2 \mathrm{H}), 2.26(\mathrm{~s}, 3 \mathrm{H}) ;{ }^{13} \mathrm{C} \mathrm{NMR}\left(176 \mathrm{MHz}, \mathrm{CDCl}_{3}\right) \delta 151.8,138.3,128.7,127.9,127.2$, 123.0, 120.3, 115.2, 33.9, 29.8, 15.9; HRMS (EI) m/z: $[\mathrm{M}]^{+}$Calcd for $\mathrm{C}_{11} \mathrm{H}_{14} \mathrm{O}$ 162.1045; Found 162.1044.<smiles>CCc1ccc(C)c(O)c1[N+](C)(C)C</smiles>

\section{3-Ethyl-2,6-dimethylphenol (49).}

3-Hydroxy-4-methyl-2H-pyran-2-one (100 mg, $0.8 \mathrm{mmol}, 2 \mathrm{eq}$ ) and 2-nitropent-2-ene ( $23 \mathrm{mg}, 0.2 \mathrm{mmol}, 1 \mathrm{eq}$ ) were subjected to the general procedure. Purification by FCC (10:1 hexanes:EtOAc) yielded 49 as a yellow oil $(24.2 \mathrm{mg}, 81 \%)$.

Data for 49: Rf: 0.54 (5:1 hexanes: EtOAc); IR (thin film) 3427, 2960, 2927, 2869, $1493 \mathrm{~cm}^{-1} ;{ }^{1} \mathrm{H}$ NMR $\left(700 \mathrm{MHz}, \mathrm{CDCl}_{3}\right) \delta 6.92(\mathrm{~d}, J=7.7 \mathrm{~Hz}, 1 \mathrm{H}), 6.70(\mathrm{~d}, J=7.7$ $\mathrm{Hz}, 1 \mathrm{H}), 4.62$ (s, $1 \mathrm{H}), 2.62$ (q, $J=7.7 \mathrm{~Hz}, 2 \mathrm{H}), 2.23$ (s, $3 \mathrm{H}), 2.21$ (s, $3 \mathrm{H}), 1.19$ (t, $J$ $=7.7 \mathrm{~Hz}, 3 \mathrm{H}) ;{ }^{13} \mathrm{C} \mathrm{NMR}\left(176 \mathrm{MHz}, \mathrm{CDCl}_{3}\right) \delta 152.1,141.7,127.7,121.1,120.2$, 120.2, 26.6, 15.9, 15.0, 11.2; HRMS (EI) m/z: [M] ${ }^{+}$Calcd for $\mathrm{C}_{10} \mathrm{H}_{14} \mathrm{O} 150.1045$; Found 150.1049 .<smiles>CCc1c(C)ccc(C)c1O</smiles>

\section{2-Ethyl-3,6-dimethylphenol (50).}

3-Hydroxy-4-methyl-2H-pyran-2-one (100 mg, $0.8 \mathrm{mmol}, 2 \mathrm{eq}$ ) and 3-nitropent-2-ene ( $23 \mathrm{mg}, 0.2 \mathrm{mmol}, 1 \mathrm{eq}$ ) were subjected to the general procedure. Purification by FCC (15:1 hexanes:EtOAc) yielded $\mathbf{5 0}$ as a yellow oil (22.5 $\mathrm{mg}, 75 \%)$.

Data for 50: Rf: 0.48 (7:1 hexanes: EtOAc); IR (thin film) 3571, 2966, 2933, 2872, $1464 \mathrm{~cm}^{-1} ;{ }^{1} \mathrm{H}$ NMR $\left(700 \mathrm{MHz}, \mathrm{CDCl}_{3}\right) \delta 6.87(\mathrm{~d}, J=7.7 \mathrm{~Hz}, 1 \mathrm{H}), 6.67(\mathrm{~d}, J=7.7$ $\mathrm{Hz}, 1 \mathrm{H}), 4.59$ (s, $1 \mathrm{H}), 2.66$ (q, $J=7.7 \mathrm{~Hz}, 2 \mathrm{H}), 2.28$ (s, $3 \mathrm{H}), 2.22$ (s, $3 \mathrm{H}), 1.15$ (t, $J$ $=7.7 \mathrm{~Hz}, 3 \mathrm{H}) ;{ }^{13} \mathrm{C} \mathrm{NMR}\left(176 \mathrm{MHz}, \mathrm{CDCl}_{3}\right) \delta 151.7,134.9,128.0,127.6,122.1$, 120.4, 19.7, 19.2, 15.8, 13.4; HRMS (EI) m/z: $[\mathrm{M}]^{+}$Calcd for $\mathrm{C}_{10} \mathrm{H}_{14} \mathrm{O} 150.1045$; Found 150.1049. 
<smiles>Cc1ccc(-c2ccccc2)c(C)c1O</smiles>

\section{2,4-Dimethyl-[1,1'-biphenyl]-3-ol (51)}

3-Hydroxy-4-methyl-2H-pyran-2-one $\quad(75.6 \mathrm{mg}, \quad 0.6 \mathrm{mmol}, 2 \mathrm{eq})$ and (2-nitroprop-1-en-1-yl)benzene (32.6 $\mathrm{mg}, 0.2 \mathrm{mmol}, 1 \mathrm{eq})$ were subjected to the general procedure. Purification by FCC (15:1 hexanes:EtOAc) yielded $\mathbf{5 1}$ as a solid (26.5 mg, 67\%). Spectroscopic data for $\mathbf{5 1}$ matched those previously reported. ${ }^{11}$<smiles>Cc1ccc(C(C)(C)C)cc1O</smiles>

\section{5-(Tert-butyl)-2-methylphenol (52)}

3-Hydroxy-4-methyl-2H-pyran-2-one (50.4 $\mathrm{mg}, \quad 0.4 \mathrm{mmol}, 2 \mathrm{eq})$ and 3,3-dimethyl-1-nitrobut-1-ene $(25.8 \mathrm{mg}, 0.2 \mathrm{mmol}, 1 \mathrm{eq})$ were subjected to the general procedure. Purification by FCC (10:1 hexanes:EtOAc) yielded $\mathbf{5 2}$ as yellow solid (25.0 mg, 76\%). Spectroscopic data for $\mathbf{5 2}$ matched those previously reported. ${ }^{17}$<smiles>CCOC(=O)c1ccc(C)c(O)c1</smiles>

\section{Ethyl 3-hydroxy-4-methylbenzoate (53).}

3-Hydroxy-4-methyl-2H-pyran-2-one (50.4 mg, $0.4 \mathrm{mmol}, 2 \mathrm{eq})$ and ethyl 3-nitroacrylate (29 mg, $0.2 \mathrm{mmol}, 1 \mathrm{eq})$ were subjected to the general procedure for 10 min. Purification by FCC (20:1 hexanes:EtOAc) yielded $\mathbf{5 3}$ as a yellow solid (22.9 $\mathrm{mg}, 7.5 \mathrm{mg}$ of the corresponding regioisomer, $\mathrm{rr}=3: 1,84 \%$ yield).

Data for 53: Rf: 0.33 (5:1 hexanes: EtOAc); mp: $79-80{ }^{\circ} \mathrm{C}$; IR (thin film) 3431, 2983, $1698 \mathrm{~cm}^{-1} ;{ }^{1} \mathrm{H}$ NMR $\left(700 \mathrm{MHz}, \mathrm{CDCl}_{3}\right) \delta 7.63(\mathrm{~d}, J=1.4 \mathrm{~Hz}, 1 \mathrm{H}), 7.52$ (dd, $J=7.7$, $1.4 \mathrm{~Hz}, 1 \mathrm{H}), 7.17$ (d, $J=7.7 \mathrm{~Hz}, 1 \mathrm{H}), 6.12$ (brs, $1 \mathrm{H}), 4.37$ (q, $J=7.0 \mathrm{~Hz}, 2 \mathrm{H}), 2.31$ $(\mathrm{s}, 3 \mathrm{H}), 1.39$ (t, $J=7.0 \mathrm{~Hz}, 3 \mathrm{H}) ;{ }^{13} \mathrm{C} \mathrm{NMR}\left(176 \mathrm{MHz}, \mathrm{CDCl}_{3}\right) \delta 167.2,154.2,130.9$, 130.3, 129.1, 121.7, 115.8, 61.2, 16.2, 14.3; HRMS (ESI) m/z: $[\mathrm{M}+\mathrm{H}]^{+}$Calcd for $\mathrm{C}_{10} \mathrm{H}_{13} \mathrm{O}_{3}$ 181.0865; Found 181.0870.<smiles>CCc1ccc(CC(C)C)c(O)c1[N+](C)(C)C</smiles>

\section{3-Ethyl-6-isobutyl-2-methylphenol (54).}

3-Hydroxy-4-isobutyl-2H-pyran-2-one (50.4 mg, $0.3 \mathrm{mmol}, 3 \mathrm{eq}$ ) and 2-nitropent-2ene $(11.5 \mathrm{mg}, 0.1 \mathrm{mmol}, 1 \mathrm{eq})$ were subjected to the general procedure. Purification 
by FCC (15:1 hexanes:EtOAc) yielded $\mathbf{5 4}$ as a yellow oil (13.4 mg, 70\%)

Data for 54: Rf: 0.48 (7:1 hexanes:EtOAc); IR (thin film) 3575, 2959, 2931, 2869, $1428 \mathrm{~cm}^{-1} ;{ }^{1} \mathrm{H}$ NMR $\left(700 \mathrm{MHz}, \mathrm{CDCl}_{3}\right) \delta 6.87(\mathrm{~d}, J=7.7 \mathrm{~Hz}, 1 \mathrm{H}), 6.71(\mathrm{~d}, J=7.7$ $\mathrm{Hz}, 1 \mathrm{H}), 4.63(\mathrm{~s}, 1 \mathrm{H}), 2.62(\mathrm{q}, J=7.0 \mathrm{~Hz}, 2 \mathrm{H}), 2.44(\mathrm{~d}, J=7.0 \mathrm{~Hz}, 2 \mathrm{H}), 2.20$ (s, 3 H), 1.90 (sept, $J=7.0 \mathrm{~Hz}, 1 \mathrm{H}$ ), 1.19 (t, $J=7.0 \mathrm{~Hz}, 3 \mathrm{H}$ ), 0.95 (d, $J=7.0 \mathrm{~Hz}, 6 \mathrm{H}$ ); ${ }^{13} \mathrm{C}$ NMR $\left(176 \mathrm{MHz}, \mathrm{CDCl}_{3}\right) \delta 151.8,141.5,128.0,123.9,121.2,120.0,39.6,28.9$, 26.7, 22.7, 14.8, 11.3; HRMS (EI) m/z: [M] Calcd for $\mathrm{C}_{13} \mathrm{H}_{20} \mathrm{O}$ 192.1514; Found 192.1523.<smiles>CCc1c(C)ccc(CC(C)C)c1O</smiles>

\section{2-Ethyl-6-isobutyl-3-methylphenol (55).}

3-Hydroxy-4-isobutyl-2H-pyran-2-one (50.4 mg, $0.3 \mathrm{mmol}, 3 \mathrm{eq})$ and 3-nitropent-2ene $(11.5 \mathrm{mg}, 0.1 \mathrm{mmol}, 1 \mathrm{eq})$ were subjected to the general procedure. Purification by FCC (50:1 hexanes:EtOAc) yielded 55 as a yellow oil $(13.5 \mathrm{mg}, 70 \%)$.

Data for 55: Rf: 0.52 (10:1 hexanes:EtOAc); IR (thin film) 3623, 3583, 2957, 2931, 2867, $1460 \mathrm{~cm}^{-1}$; ${ }^{1} \mathrm{H}$ NMR $\left(700 \mathrm{MHz}, \mathrm{CDCl}_{3}\right) \delta 6.82(\mathrm{~d}, J=7.0 \mathrm{~Hz}, 1 \mathrm{H}), 6.69(\mathrm{~d}, J=$ $7.0 \mathrm{~Hz}, 1 \mathrm{H}), 4.63(\mathrm{~s}, 1 \mathrm{H}), 2.66(\mathrm{q}, J=7.0 \mathrm{~Hz}, 2 \mathrm{H}), 2.43$ (d, J=7.0 Hz, $2 \mathrm{H}), 2.29$ (s, $3 \mathrm{H}), 1.90$ (sept, $J=7.0 \mathrm{~Hz}, 1 \mathrm{H}$ ), 1.15 (t, $J=7.0 \mathrm{~Hz}, 3 \mathrm{H}), 0.95$ (d, $J=7.0 \mathrm{~Hz}, 6 \mathrm{H}$ ); ${ }^{13} \mathrm{C}$ NMR $\left(176 \mathrm{MHz}, \mathrm{CDCl}_{3}\right) \delta 151.5,134.9,128.2,127.9,124.2,121.9,39.6,29.0$, 22.7, 19.8, 19.3, 13.5; HRMS (EI) m/z: [M] Calcd for $\mathrm{C}_{13} \mathrm{H}_{20} \mathrm{O}$ 192.1514; Found 192.1522 .<smiles>CCOC(=O)c1ccc(C)c(O)c1C</smiles>

\section{Ethyl 3-hydroxy-2,4-dimethylbenzoate (56).}

3-Hydroxy-4-methyl-2H-pyran-2-one (189 mg, $1.5 \mathrm{mmol}, 1.5 \mathrm{eq})$ and ethyl 3-nitrobut-2-enoate $(159 \mathrm{mg}, 1 \mathrm{mmol}, 1 \mathrm{eq})$ were subjected to the general procedure. Purification by FCC (20:1 hexanes:EtOAc) yielded $\mathbf{5 6}$ as a yellow solid (128.2 $\mathrm{mg}$ of $\mathbf{5 6}, 45.2 \mathrm{mg}$ of the corresponding regioisomer, $\mathrm{rr}=2.8: 1,89 \%$ yield).

Data for 56: Rf: 0.42 (5:1 hexanes:EtOAc); mp: $35-37^{\circ} \mathrm{C}$; IR (thin film) 3473, 2980, 2926, $1697 \mathrm{~cm}^{-1} ;{ }^{1} \mathrm{H}$ NMR $\left(700 \mathrm{MHz}, \mathrm{CDCl}_{3}\right) \delta 7.36(\mathrm{~d}, J=7.7 \mathrm{~Hz}, 1 \mathrm{H}), 6.99(\mathrm{~d}, J=$ $7.7 \mathrm{~Hz}, 1 \mathrm{H}), 5.26$ (s, $1 \mathrm{H}), 4.34$ (q, $J=7.0 \mathrm{~Hz}, 2 \mathrm{H}), 2.47$ (s, $3 \mathrm{H}), 2.27$ (s, $3 \mathrm{H}), 1.38$ $(\mathrm{t}, J=7.0 \mathrm{~Hz}, 3 \mathrm{H}) ;{ }^{13} \mathrm{C} \mathrm{NMR}\left(176 \mathrm{MHz}, \mathrm{CDCl}_{3}\right) \delta 168.3,152.7,129.5,127.7,127.6$, 124.8, 122.3, 60.9, 16.5, 14.3, 12.7; HRMS (ESI) m/z: $[\mathrm{M}+\mathrm{H}]^{+}$Calcd for $\mathrm{C}_{11} \mathrm{H}_{15} \mathrm{O}_{3}$ 195.1021; Found 195.1018. 
<smiles>CCOC(=O)c1ccc(C)c(O)c1CC</smiles>

Ethyl 2-ethyl-3-hydroxy-4-methylbenzoate (57).

3-Hydroxy-4-methyl-2H-pyran-2-one (50.4 $\mathrm{mg}, 0.4 \mathrm{mmol}, 2 \mathrm{eq})$ and ethyl 3-nitropent-2-enoate $(34.6 \mathrm{mg}, 0.2 \mathrm{mmol}, 1 \mathrm{eq})$ were subjected to the general procedure. Purification by FCC (20:1 hexanes:EtOAc) yielded 57 as a yellow solid (28.5 $\mathrm{mg}$ of 57, $9.6 \mathrm{mg}$ of $\mathbf{5 8}, \mathrm{rr}=3: 1$, yield: $91 \%$ ).

Data for 57: Rf: 0.30 (5:1 hexanes:EtOAc); mp: $38-40{ }^{\circ} \mathrm{C}$; IR (thin film) 3486, 2978, 2936, 2875, 1699, $1271 \mathrm{~cm}^{-1}$; ${ }^{1} \mathrm{H}$ NMR $\left(700 \mathrm{MHz}, \mathrm{CDCl}_{3}\right) \delta 7.34(\mathrm{~d}, J=7.7 \mathrm{~Hz}, 1 \mathrm{H})$, $7.01(\mathrm{~d}, J=7.7 \mathrm{~Hz}, 1 \mathrm{H}), 5.02(\mathrm{~s}, 1 \mathrm{H}), 4.34(\mathrm{q}, J=7.0 \mathrm{~Hz}, 2 \mathrm{H}), 2.95(\mathrm{q}, J=7.0 \mathrm{~Hz}$, $2 \mathrm{H}), 2.28(\mathrm{~s}, 3 \mathrm{H}), 1.38(\mathrm{t}, J=7.0 \mathrm{~Hz}, 3 \mathrm{H}), 1.23(\mathrm{t}, J=7.0 \mathrm{~Hz}, 3 \mathrm{H}) ;{ }^{13} \mathrm{C}$ NMR $(176$ $\left.\mathrm{MHz}, \mathrm{CDCl}_{3}\right) \delta 168.1,152.3,130.6,129.3,127.8,127.5,122.4,60.8,20.4,16.4,14.3$; HRMS (ESI) m/z: [M+ H] $]^{+}$Calcd for $\mathrm{C}_{12} \mathrm{H}_{17} \mathrm{O}_{3} 209.1178$; Found 209.1178.<smiles>CCOC(=O)c1c(CC)ccc(C)c1O</smiles>

Ethyl 6-ethyl-2-hydroxy-3-methylbenzoate (58).

3-Hydroxy-4-methyl-2H-pyran-2-one (50.4 mg, $0.4 \mathrm{mmol}, 2 \mathrm{eq})$ and ethyl 2-nitropent-2-enoate (34.6 mg, $0.2 \mathrm{mmol}, 1 \mathrm{eq})$ were subjected to the general procedure for $7 \mathrm{~h}$. Purification by FCC (20:1 hexanes:EtOAc) yielded $\mathbf{5 8}$ as a yellow oil (15 mg, 36\%),

Data for 58: Rf: 0.75 (5:1 hexanes:EtOAc); IR (thin film) 2978, 2932, 1655, 1248 $\mathrm{cm}^{-1} ;{ }^{1} \mathrm{H} \mathrm{NMR}\left(700 \mathrm{MHz}, \mathrm{CDCl}_{3}\right) \delta 11.51(\mathrm{~d}, J=0.7 \mathrm{~Hz}, 1 \mathrm{H}), 7.18(\mathrm{~d}, J=7.7 \mathrm{~Hz}, 1$ $\mathrm{H}), 6.65(\mathrm{~d}, J=7.7 \mathrm{~Hz}, 1 \mathrm{H}), 4.44(\mathrm{q}, J=7.0 \mathrm{~Hz}, 2 \mathrm{H}), 2.91$ (q, $J=7.0 \mathrm{~Hz}, 2 \mathrm{H}), 2.22$ $(\mathrm{s}, 3 \mathrm{H}), 1.44(\mathrm{t}, J=7.0 \mathrm{~Hz}, 3 \mathrm{H}), 1.20(\mathrm{t}, J=7.0 \mathrm{~Hz}, 3 \mathrm{H}) ;{ }^{13} \mathrm{C}$ NMR $(176 \mathrm{MHz}$, $\left.\mathrm{CDCl}_{3}\right) \delta 172.0,160.9,144.8,135.1,124.2,120.9,111.2,61.6,29.6,16.4,15.9,14.0$; HRMS (ESI) m/z: [M+ H] $]^{+}$Calcd for $\mathrm{C}_{12} \mathrm{H}_{17} \mathrm{O}_{3} 209.1178$; Found 209.1182.<smiles></smiles>

2-Methyl-5-(2-((triisopropylsilyl)oxy)ethyl)phenol (59).

3-Hydroxy-4-methyl-2H-pyran-2-one (50.4 mg, $0.4 \mathrm{mmol}, 2 \mathrm{eq})$ and triisopropyl((4nitrobut-3- en-1-yl)oxy)silane (54.6 mg, $0.2 \mathrm{mmol}, 1 \mathrm{eq}$ ) were subjected to the general procedure. Purification by FCC (20:1 hexanes:EtOAc) yielded 59 as a yellow oil (48.2 mg, 78\%). 
Data for 59: Rf: 0.56 (5:1 hexanes:EtOAc); IR (thin film) 3384, 2953, 2892, 2866, $1462 \mathrm{~cm}^{-1} ;{ }^{1} \mathrm{H}$ NMR $\left(700 \mathrm{MHz}, \mathrm{CDCl}_{3}\right) \delta 7.03(\mathrm{~d}, J=7.7 \mathrm{~Hz}, 1 \mathrm{H}), 6.71(\mathrm{~d}, J=7.7$ $\mathrm{Hz}, 1 \mathrm{H}), 6.65(\mathrm{~s}, 1 \mathrm{H}), 4.76(\mathrm{~s}, 1 \mathrm{H}), 3.85(\mathrm{t}, J=7.7 \mathrm{~Hz}, 2 \mathrm{H}), 2.80(\mathrm{t}, J=7.7 \mathrm{~Hz}, 2$ $\mathrm{H}), 2.22(\mathrm{~s}, 3 \mathrm{H}), 1.13-1.07(\mathrm{~m}, 3 \mathrm{H}), 1.06(\mathrm{~d}, J=6.3 \mathrm{~Hz}, 18 \mathrm{H}) ;{ }^{13} \mathrm{C}$ NMR $(176$ $\left.\mathrm{MHz}, \mathrm{CDCl}_{3}\right) \delta 153.6,138.3,130.8,121.4,121.3,115.7,65.0,39.3,18.0,15.4,12.0$; HRMS (ESI) m/z: [M + Na] $]^{+}$Calcd for $\mathrm{C}_{18} \mathrm{H}_{32} \mathrm{O}_{2} \mathrm{SiNa} 331.2069$; Found 331.2066.<smiles>CCc1cc(O)cc(CCc2ccccc2)c1-c1ccccc1</smiles>

\section{2-(2-((Tert-butyldimethylsilyl)oxy)ethyl)-6-ethyl-[1,1'-biphenyl]-4-ol (60).}

5-Ethyl-3-hydroxy-6-phenyl-2H-pyran-2-one (21.6 mg, $0.1 \mathrm{mmol}, 1 \mathrm{eq})$ and tert-butyldimethyl ((4-nitrobut-3-en-1-yl)oxy)silane (46.2 mg, $0.2 \mathrm{mmol}, 2 \mathrm{eq})$ were subjected to the general procedure. Purification by FCC (20:1 hexanes:EtOAc) yielded $\mathbf{6 0}$ as a yellow solid (13.2 $\mathrm{mg}, 37 \%$ ).

Data for 60: Rf: 0.42 (5:1 hexanes:EtOAc); mp: $74-78^{\circ} \mathrm{C}$; IR (thin film) 3339, 2957, 2929, 2857, 1609, $1456 \mathrm{~cm}^{-1} ;{ }^{1} \mathrm{H} \mathrm{NMR}\left(700 \mathrm{MHz}, \mathrm{CDCl}_{3}\right) \delta 7.39$ (t, $\left.J=7.0 \mathrm{~Hz}, 2 \mathrm{H}\right)$, $7.33(\mathrm{t}, J=7.0 \mathrm{~Hz}, 1 \mathrm{H}), 7.14(\mathrm{~d}, J=7.0 \mathrm{~Hz}, 2 \mathrm{H}), 6.64(\mathrm{~d}, J=2.8 \mathrm{~Hz}, 2 \mathrm{H}), 4.76(\mathrm{~s}, 1$ H), 3.56 (t, $J=7.7 \mathrm{~Hz}, 2 \mathrm{H}), 2.53(\mathrm{t}, J=7.7 \mathrm{~Hz}, 2 \mathrm{H}), 2.27$ (q, $J=7.7 \mathrm{~Hz}, 2 \mathrm{H}), 1.00$ $(\mathrm{t}, J=7.7 \mathrm{~Hz}, 3 \mathrm{H}), 0.81(\mathrm{~s}, 9 \mathrm{H}),-0.13(\mathrm{~s}, 6 \mathrm{H}) ;{ }^{13} \mathrm{C} \mathrm{NMR}\left(176 \mathrm{MHz}, \mathrm{CDCl}_{3}\right) \delta 154.5$, 144.3, 139.9, 138.3, 134.3, 130.1, 128.2, 126.7, 114.0, 112.9, 64.1, 37.4, 26.9, 26.0, 18.3, 15.4, -5.4; HRMS (ESI) m/z: $[\mathrm{M}+\mathrm{Na}]^{+}$Calcd for $\mathrm{C}_{22} \mathrm{H}_{32} \mathrm{O}_{2} \mathrm{SiNa}$ 379.2069; Found 379.2063.<smiles>CCc1cc(O)cc(CC)c1-c1ccccc1</smiles>

\section{2,6-Diethyl-[1,1'-biphenyl]-4-ol (61).}

5-Ethyl-3-hydroxy-6-phenyl-2H-pyran-2-one (21.6 $\mathrm{mg}, 0.1 \mathrm{mmol}, 1 \mathrm{eq})$ and 1-nitrobut-1-ene $(80.8 \mathrm{mg}, 0.8 \mathrm{mmol}, 8 \mathrm{eq})$ were subjected to the general procedure with hydroquinone $(1.1 \mathrm{mg}, 0.01 \mathrm{mmol}, 0.1 \mathrm{eq})$ added as an additional radical inhibitor. Purification by FCC (20:1 hexanes:EtOAc) yielded $\mathbf{6 1}$ as a yellow solid (11.2 mg, $50 \%)$.

Data for 61: Rf: 0.36 (5:1 hexanes:EtOAc); mp: $99-101{ }^{\circ} \mathrm{C}$; IR (thin film) 3357, 2965, 2933, 2871, 1609, $1454 \mathrm{~cm}^{-1} ;{ }^{1} \mathrm{H}$ NMR (700 MHz, $\left.\mathrm{CDCl}_{3}\right) \delta 7.40$ (dt, $J=7.7$, $1.4 \mathrm{~Hz}, 2 \mathrm{H}), 7.34(\mathrm{tt}, J=7.7,1.4 \mathrm{~Hz}, 1 \mathrm{H}), 7.16(\mathrm{dd}, J=7.7,1.4 \mathrm{~Hz}, 2 \mathrm{H}), 6.64$ (s, 2 $\mathrm{H}), 4.78(\mathrm{~s}, 2 \mathrm{H}), 2.29(\mathrm{q}, J=7.7 \mathrm{~Hz}, 4 \mathrm{H}), 1.02(\mathrm{t}, J=7.7 \mathrm{~Hz}, 6 \mathrm{H}) ;{ }^{13} \mathrm{C}$ NMR $(176$ $\left.\mathrm{MHz}, \mathrm{CDCl}_{3}\right) \delta 154.7,144.1,140.2,133.7,130.1,128.0,126.6,112.3,26.9,15.4$; 
HRMS (EI) m/z: [M] $]^{+}$Calcd for $\mathrm{C}_{16} \mathrm{H}_{18} \mathrm{O}$ 226.1358; Found 226.1365.<smiles>CCc1cc(-c2ccccc2)c(CC)cc1O</smiles>

\section{2,5-Diethyl-[1,1'-biphenyl]-4-ol (62).}

5-Ethyl-3-hydroxy-6-phenyl-2H-pyran-2-one (43.2 $\mathrm{mg}, 0.2 \mathrm{mmol}, 1 \mathrm{eq})$ and 2-nitrobut-1-ene $(60.6 \mathrm{mg}, 0.6 \mathrm{mmol}, 3 \mathrm{eq})$ were subjected to the general procedure at $180{ }^{\circ} \mathrm{C}$ for $7 \mathrm{~h}$. Purification by FCC (20:1 hexanes:EtOAc) yielded $\mathbf{6 2}$ as a yellow solid (22.5 mg, 50\%).

Data for 62: Rf: 0.39 (5:1 hexanes:EtOAc); mp: $65-67^{\circ} \mathrm{C}$; IR (thin film) 3400, 2965, 2930, 2872, 1615, $1488 \mathrm{~cm}^{-1}$; ${ }^{1} \mathrm{H}$ NMR $\left(700 \mathrm{MHz}, \mathrm{CDCl}_{3}\right) \delta 7.40(\mathrm{t}, J=7.0 \mathrm{~Hz}, 2 \mathrm{H})$, $7.34-7.31(\mathrm{~m}, 3 \mathrm{H}), 7.01(\mathrm{~s}, 1 \mathrm{H}), 6.74(\mathrm{~s}, 1 \mathrm{H}), 4.74(\mathrm{~s}, 1 \mathrm{H}), 2.66(\mathrm{q}, J=7.7 \mathrm{~Hz}, 2$ H), $2.55(\mathrm{q}, J=7.7 \mathrm{~Hz}, 2 \mathrm{H}), 1.27(\mathrm{t}, J=7.7 \mathrm{~Hz}, 3 \mathrm{H}), 1.10(\mathrm{t}, J=7.7 \mathrm{~Hz}, 3 \mathrm{H}) ;{ }^{13} \mathrm{C}$ NMR $\left(176 \mathrm{MHz}, \mathrm{CDCl}_{3}\right) \delta 152.7,141.9,140.5,134.4,130.9,129.5,128.0,126.9$, 126.5, 115.0, 25.8, 22.6, 15.6, 14.1; HRMS (EI) m/z: $[\mathrm{M}]^{+}$Calcd for $\mathrm{C}_{16} \mathrm{H}_{18} \mathrm{O}$ 226.1358; Found 226.1369.<smiles>CCc1cc(O)c(Br)cc1-c1ccccc1</smiles>

\section{5-Benzyl-2-ethyl-[1,1'-biphenyl]-4-ol (63).}

5-Ethyl-3-hydroxy-6-phenyl-2H-pyran-2-one (86.4 $\mathrm{mg}, 0.4 \mathrm{mmol}, 2 \mathrm{eq})$ and (2-nitroallyl) benzene $(32.6 \mathrm{mg}, 0.2 \mathrm{mmol}, 1 \mathrm{eq})$ were subjected to the general procedure for $6 \mathrm{~h}$. Purification by FCC (10:1 hexanes:EtOAc) yielded 63 as a yellow solid (38.2 mg, 66\%).

Data for 63: Rf: 0.39 (5:1 hexanes:EtOAc); mp: $93-97{ }^{\circ} \mathrm{C}$; IR (thin film) 3534, 3027, 2966, 2930, 1614, $1488 \mathrm{~cm}^{-1}$; ${ }^{1} \mathrm{H}$ NMR $\left(700 \mathrm{MHz}, \mathrm{CDCl}_{3}\right) \delta 7.40(\mathrm{dt}, J=7.0,1.4 \mathrm{~Hz}$, $2 \mathrm{H}), 7.34$ (tt, J = 7.0, $1.4 \mathrm{~Hz}, 1 \mathrm{H}), 7.32-7.29(\mathrm{~m}, 6 \mathrm{H}), 7.23(\mathrm{tt}, J=7.7,1.4 \mathrm{~Hz}, 1$ H), $7.03(\mathrm{~s}, 1 \mathrm{H}), 6.76(\mathrm{~s}, 1 \mathrm{H}), 4.73(\mathrm{~s}, 1 \mathrm{H}), 4.02(\mathrm{~s}, 2 \mathrm{H}), 2.57(\mathrm{q}, J=7.7 \mathrm{~Hz}, 2 \mathrm{H})$, $1.12(\mathrm{t}, J=7.7 \mathrm{~Hz}, 3 \mathrm{H}) ;{ }^{13} \mathrm{C} \mathrm{NMR}\left(176 \mathrm{MHz}, \mathrm{CDCl}_{3}\right) \delta 153.1,141.7,141.4,140.0$, 134.5, 132.6, 129.6, 128.7, 128.7, 128.0, 126.5, 126.4, 124.1, 115.7, 36.2, 25.9, 15.6; HRMS (EI) m/z: [M] $]^{+}$Calcd for $\mathrm{C}_{21} \mathrm{H}_{20} \mathrm{O}$ 288.1514; Found 288.1526.<smiles>CCOC(=O)c1cc(O)cc(CC)c1-c1ccccc1</smiles> 
Ethyl 6-ethyl-4-hydroxy-[1,1'-biphenyl]-2-carboxylate (64).

5-Ethyl-3-hydroxy-6-phenyl-2H-pyran-2-one (43.2 $\mathrm{mg}, 0.2 \mathrm{mmol}, 1 \mathrm{eq})$ and 1-nitropent-1-ene (58 $\mathrm{mg}, 0.4 \mathrm{mmol}, 2 \mathrm{eq})$ were subjected to the general procedure. Purification by FCC (20:1 hexanes:EtOAc) yielded 64 as a yellow solid (14.1 $\mathrm{mg}$ of 64, $10.5 \mathrm{mg}$ of the corresponding regioisomer, $\mathrm{rr}=1.4: 1,46 \%$ yield).

Data for 64: Rf: 0.33 (5:1 hexanes:EtOAc); mp: $101-103{ }^{\circ} \mathrm{C}$; IR (thin film) 3386, 2970, 2933, 1703, $1609 \mathrm{~cm}^{-1}$; ${ }^{1} \mathrm{H}$ NMR $\left(700 \mathrm{MHz}, \mathrm{CDCl}_{3}\right) \delta 7.35$ (tt, $J=7.0,1.4 \mathrm{~Hz}$, $2 \mathrm{H}), 7.32$ (tt, $J=7.0,1.4 \mathrm{~Hz}, 1 \mathrm{H}), 7.17(\mathrm{~d}, J=2.7 \mathrm{~Hz}, 1 \mathrm{H}), 7.16(\mathrm{td}, J=7.0,1.4 \mathrm{~Hz}$, $2 \mathrm{H}), 6.93(\mathrm{~d}, J=2.8 \mathrm{~Hz}, 1 \mathrm{H}), 5.32(\mathrm{brs}, 1 \mathrm{H}), 3.95(\mathrm{q}, J=7.0 \mathrm{~Hz}, 2 \mathrm{H}), 2.38(\mathrm{q}, J=$ $7.7 \mathrm{~Hz}, 2 \mathrm{H}), 1.02$ (t, $J=7.0 \mathrm{~Hz}, 3 \mathrm{H}), 0.88(\mathrm{t}, J=7.7 \mathrm{~Hz}, 3 \mathrm{H}) ;{ }^{13} \mathrm{C} \mathrm{NMR}(176 \mathrm{MHz}$, $\left.\mathrm{CDCl}_{3}\right) \delta 168.7,154.6,145.2,139.8,133.8,133.3,129.5,127.7,126.7,118.3,113.6$, 60.9, 26.5, 15.3, 13.5; HRMS (ESI) m/z: $[\mathrm{M}+\mathrm{H}]^{+}$Calcd for $\mathrm{C}_{17} \mathrm{H}_{19} \mathrm{O}_{3} 271.1334$; Found 271.1322.<smiles>CCc1cc(O)cc(C)c1-c1ccccc1</smiles>

\section{2-Ethyl-6-methyl-[1,1'-biphenyl]-4-ol (65).}

3-Hydroxy-5-methyl-6-phenyl-2H-pyran-2-one (20.2 mg, $0.1 \mathrm{mmol}, 1 \mathrm{eq})$ and 1-nitrobut-1-ene $(50.5 \mathrm{mg}, 0.5 \mathrm{mmol}, 5 \mathrm{eq})$ were subjected to the general procedure, except 2,6-di-tertbutyl phenol (10.3 $\mathrm{mg}, 0.05 \mathrm{mmol}, 0.5 \mathrm{eq})$ was used as a radical inhibitor instead of BHT. Purification by FCC (20:1 hexanes:EtOAc) yielded $\mathbf{6 5}$ as a yellow solid (10.4 mg, 49\%).

Data for 65: Rf: 0.45 (5:1 hexanes:EtOAc); mp: $89-93{ }^{\circ} \mathrm{C}$; IR (thin film) 3306, 2968, 2926, 1593, 1460, $1299 \mathrm{~cm}^{-1}$; ${ }^{1} \mathrm{H} \mathrm{NMR}\left(700 \mathrm{MHz}, \mathrm{CDCl}_{3}\right) \delta 7.40(\mathrm{t}, J=7.7 \mathrm{~Hz}, 2 \mathrm{H})$, 7.33 (t, $J=7.7 \mathrm{~Hz}, 1 \mathrm{H}), 7.14(\mathrm{~d}, J=7.7 \mathrm{~Hz}, 2 \mathrm{H}), 6.63(\mathrm{~d}, J=2.1 \mathrm{~Hz}, 1 \mathrm{H}), 6.60(\mathrm{~d}, J$ $=2.1 \mathrm{~Hz}, 1 \mathrm{H}), 4.65(\mathrm{~s}, 1 \mathrm{H}), 2.30(\mathrm{q}, J=7.7 \mathrm{~Hz}, 2 \mathrm{H}), 1.97(\mathrm{~s}, 3 \mathrm{H}), 1.02(\mathrm{t}, J=7.7$ $\mathrm{Hz}, 3 \mathrm{H}) ;{ }^{13} \mathrm{C} \mathrm{NMR}\left(176 \mathrm{MHz}, \mathrm{CDCl}_{3}\right) \delta 154.4,144.0,140.5,137.9,134.2,129.9$, 128.3, 126.5, 113.9, 112.2, 26.8, 21.0, 15.4; HRMS (ESI) m/z: $[\mathrm{M}+\mathrm{H}]^{+}$Calcd for $\mathrm{C}_{15} \mathrm{H}_{17} \mathrm{O} 213.1279$; Found 213.1274.<smiles>CCc1cc(-c2ccccc2)c(C)cc1O</smiles>

\section{5-Ethyl-2-methyl-[1,1'-biphenyl]-4-ol (66).}

3-Hydroxy-5-methyl-6-phenyl-2H-pyran-2-one (20.2 $\mathrm{mg}, 0.1 \mathrm{mmol}, 1 \mathrm{eq})$ and 2-nitrobut-1-ene $(50.5 \mathrm{mg}, 0.5 \mathrm{mmol}, 5 \mathrm{eq})$ were subjected to the general procedure, except 2,6-di-tertbutyl phenol $(10.3 \mathrm{mg}, 0.05 \mathrm{mmol}, 0.5 \mathrm{eq})$ was used as a radical 
inhibitor instead of BHT. Purification by FCC (20:1 hexanes:EtOAc) yielded 66 as a yellow solid (9.5 mg, 45\%).

Data for 66: Rf: 0.40 (5:1 hexanes:EtOAc); mp: $56-56{ }^{\circ} \mathrm{C}$; IR (thin film) 3406, 2965, 2929, $1615 \mathrm{~cm}^{-1} ;{ }_{1}^{1} \mathrm{H}$ NMR (700 MHz, $\left.\mathrm{CDCl}_{3}\right) \delta 7.40(\mathrm{t}, J=7.7 \mathrm{~Hz}, 2 \mathrm{H}), 7.33-7.31$ $(\mathrm{m}, 3 \mathrm{H}), 7.03(\mathrm{~s}, 1 \mathrm{H}), 6.70(\mathrm{~s}, 1 \mathrm{H}), 4.69(\mathrm{~s}, 1 \mathrm{H}), 2.65$ (q, $J=7.7 \mathrm{~Hz}, 2 \mathrm{H}), 2.22$ (s, 3 $\mathrm{H}), 1.26(\mathrm{t}, J=7.7 \mathrm{~Hz}, 3 \mathrm{H}) ;{ }^{13} \mathrm{C} \mathrm{NMR}\left(176 \mathrm{MHz}, \mathrm{CDCl}_{3}\right) \delta 152.4,141.8,134.7$, 134.1, 130.9, 129.5, 128.0, 127.1, 126.4, 116.8, 22.6, 20.1, 14.2; HRMS (ESI) m/z: $[\mathrm{M}+\mathrm{H}]^{+}$Calcd for $\mathrm{C}_{15} \mathrm{H}_{17} \mathrm{O} 213.1255$; Found 213.1260.<smiles>CCCc1cc(O)cc(C)c1-c1ccccc1</smiles>

\section{2-Methyl-6-propyl-[1,1'-biphenyl]-4-ol (67).}

3-Hydroxy-5-methyl-6-phenyl-2H-pyran-2-one (20.2 mg, $0.1 \mathrm{mmol}, 1 \mathrm{eq})$ and 1-nitropent-1-ene (58 $\mathrm{mg}, 0.5 \mathrm{mmol}, 5 \mathrm{eq})$ were subjected to the general procedure, except 2,6-di-tertbutyl phenol $(10.3 \mathrm{mg}, 0.05 \mathrm{mmol} 0.5 \mathrm{eq})$ was used as a radical inhibitor instead of BHT. Purification by FCC (20:1 hexanes:EtOAc) yielded 67 as a yellow solid (11.7 mg, 55\%).

Data for 67: Rf: 0.39 (5:1 hexanes:EtOAc); mp: $51-54{ }^{\circ} \mathrm{C}$; IR (thin film) 3338, 2959, 2929, 2870, 1608, 1592, 1455, 1307, $1151 \mathrm{~cm}^{-1} ;{ }^{1} \mathrm{H} \mathrm{NMR}\left(700 \mathrm{MHz}, \mathrm{CDCl}_{3}\right) \delta 7.40(\mathrm{t}$, $J=7.7 \mathrm{~Hz}, 2 \mathrm{H}), 7.33(\mathrm{tt}, J=7.7,1.4 \mathrm{~Hz}, 1 \mathrm{H}), 7.13(\mathrm{dd}, J=1.4,7.7 \mathrm{~Hz}, 2 \mathrm{H}), 6.62(\mathrm{~d}$, $J=2.8 \mathrm{~Hz}, 1 \mathrm{H}), 6.61(\mathrm{~d}, J=2.1 \mathrm{~Hz}, 1 \mathrm{H}), 4.77(\mathrm{~s}, 1 \mathrm{H}), 2.26(\mathrm{t}, J=7.7 \mathrm{~Hz}, 2 \mathrm{H})$, 1.97 (s, $3 \mathrm{H}), 1.43$ (sext, $J=7.7 \mathrm{~Hz}, 2 \mathrm{H}), 0.77$ (t, $J=7.7 \mathrm{~Hz}, 3 \mathrm{H}) ;{ }^{13} \mathrm{C}$ NMR $(176$ $\left.\mathrm{MHz}, \mathrm{CDCl}_{3}\right) \delta 154.2,142.4,140.6,137.9,134.5,129.9,128.2,126.5,114.0,112.9$, 35.7, 24.2, 21.1, 14.1; HRMS (EI) m/z: [M] Calcd for $\mathrm{C}_{16} \mathrm{H}_{18} \mathrm{O}$ 226.1358; Found 226.1354 .<smiles>CCc1cc(O)cc(P)c1-c1ccccc1</smiles>

\section{2-Ethyl-6-propyl-[1,1'-biphenyl]-4-ol (68).}

5-Ethyl-3-hydroxy-6-phenyl-2H-pyran-2-one (21.6 $\mathrm{mg}, 0.1 \mathrm{mmol}, 1 \mathrm{eq})$ and 1-nitropent-1-ene ( $58 \mathrm{mg}, 0.5 \mathrm{mmol}, 5 \mathrm{eq})$ were subjected to the general procedure. Purification by FCC (20:1 hexanes:EtOAc) yielded $\mathbf{6 8}$ as a yellow solid (13.2 mg, $55 \%)$.

Data for 68: Rf: 0.45 (5:1 hexanes:EtOAc); mp: $73-75^{\circ} \mathrm{C}$; IR (thin film) 3306, 2960, 2929, 2869, 1593, $1455 \mathrm{~cm}^{-1}$; ${ }^{1} \mathrm{H} \mathrm{NMR}\left(400 \mathrm{MHz}, \mathrm{CDCl}_{3}\right) \delta 7.41-7.37$ (m, $2 \mathrm{H}$ ), 
$7.33(\mathrm{tt}, J=6.4,1.6 \mathrm{~Hz}, 1 \mathrm{H}), 7.14(\mathrm{tt}, J=6.4,1.6 \mathrm{~Hz}, 2 \mathrm{H}), 6.63(\mathrm{~d}, J=2.8 \mathrm{~Hz}, 1 \mathrm{H})$, $6.61(\mathrm{~d}, J=2.8 \mathrm{~Hz}, 1 \mathrm{H}), 4.68(\mathrm{~s}, 1 \mathrm{H}), 2.31-2.21(\mathrm{~m}, 4 \mathrm{H}), 1.42(\mathrm{sext}, J=7.2 \mathrm{~Hz}, 2$ $\mathrm{H}), 1.01$ (t, $J=7.6 \mathrm{~Hz}, 3 \mathrm{H}), 0.76(\mathrm{t}, J=7.2 \mathrm{~Hz}, 3 \mathrm{H}) ;{ }^{13} \mathrm{C} \mathrm{NMR}\left(100 \mathrm{MHz}, \mathrm{CDCl}_{3}\right) \delta$ $154.5,144.1,142.5,140.2,134.0,130.2,128.0,126.5,112.9,112.3,35.8,26.9,24.2$, 15.3, 14.0; HRMS (ESI) $\mathrm{m} / \mathrm{z}$ : $[\mathrm{M}+\mathrm{H}]^{+}$Calcd for $\mathrm{C}_{17} \mathrm{H}_{21} \mathrm{O}$ 241.1592; Found 241.1599 .<smiles>CCOC(=O)c1cc(O)cc(C)c1-c1ccccc1</smiles>

\section{Ethyl 4-hydroxy-6-methyl-[1,1'-biphenyl]-2-carboxylate (69).}

3-Hydroxy-5-methyl-6-phenyl-2H-pyran-2-one (40.4 mg, $0.2 \mathrm{mmol}, 1 \mathrm{eq})$ and ethyl 3-nitroacrylate $(58 \mathrm{mg}, 0.4 \mathrm{mmol}, 2 \mathrm{eq})$ were subjected to the general procedure. Purification by FCC (20:1 hexanes:EtOAc) yielded 69 as a yellow solid (12 mg of 69, $10 \mathrm{mg}$ of the corresponding regioisomer, $\mathrm{rr}=1.2: 1,43 \%$ yield).

Data for 69: Rf: 0.24 (5:1 hexanes:EtOAc); mp: $117-121{ }^{\circ} \mathrm{C}$; IR (thin film) 3386, 2981, 2925, 1705, 1611, 1452, $1317 \mathrm{~cm}^{-1} ;{ }^{1} \mathrm{H}$ NMR (700 MHz, $\left.\mathrm{CDCl}_{3}\right) \delta 7.36(\mathrm{t}, J=$ $7.7 \mathrm{~Hz}, 2 \mathrm{H}), 7.31$ (tt, $J=7.7,1.4 \mathrm{~Hz}, 1 \mathrm{H}), 7.19$ (d, J = 2.8 Hz, $1 \mathrm{H}), 7.15-7.13$ (m, 2 H), $6.90(\mathrm{~d}, J=2.8 \mathrm{~Hz}, 1 \mathrm{H}), 3.95(\mathrm{q}, J=7.0 \mathrm{~Hz}, 2 \mathrm{H}), 2.06(\mathrm{~s}, 3 \mathrm{H}), 0.88$ (t, $J=7.0$ $\mathrm{Hz}, 3 \mathrm{H}) ;{ }^{13} \mathrm{C} \mathrm{NMR}\left(176 \mathrm{MHz}, \mathrm{CDCl}_{3}\right) \delta 168.7,154.4,140.2,139.1,134.4,133.0$, $129.2,127.9,126.7,120.0,113.7,60.9,20.9,13.5 ; \mathrm{HRMS}(\mathrm{ESI}) \mathrm{m} / \mathrm{z}:[\mathrm{M}+\mathrm{Na}]^{+}$ Calcd for $\mathrm{C}_{16} \mathrm{H}_{16} \mathrm{O}_{3} \mathrm{Na} 279.0997$; Found 279.0992.<smiles>CCOC(=O)c1c(C)c(O)cc(CC)c1-c1ccccc1</smiles>

\section{Ethyl 6-ethyl-4-hydroxy-3-methyl-[1,1'-biphenyl]-2-carboxylate (70).}

5-Ethyl-3-hydroxy-6-phenyl-2H-pyran-2-one (86.4 mg, $0.4 \mathrm{mmol}, 2 \mathrm{eq})$ and ethyl 3-nitrobut-2-enoate $(31.8 \mathrm{mg}, 0.2 \mathrm{mmol}, 1 \mathrm{eq})$ were subjected to the general procedure for 5 days. Purification by FCC (10:1 hexanes:EtOAc) yielded 70 as a yellow solid (21.6 mg, 38\%).

Data for 70: Rf: 0.42 (5:1 hexanes:EtOAc); mp: $91-95{ }^{\circ} \mathrm{C}$; IR (thin film) 3396, 2968, 1699, 1600, 1463, $1309 \mathrm{~cm}^{-1} ;{ }^{1} \mathrm{H} \mathrm{NMR}\left(700 \mathrm{MHz}, \mathrm{CDCl}_{3}\right) \delta 7.35(\mathrm{t}, J=7.7 \mathrm{~Hz}, 2 \mathrm{H})$, $7.30(\mathrm{tt}, J=7.7,1.4 \mathrm{~Hz}, 1 \mathrm{H}), 7.21(\mathrm{dd}, J=7.7,1.4 \mathrm{~Hz}, 2 \mathrm{H}), 6.75(\mathrm{~s}, 1 \mathrm{H}), 5.29$ (s, 1 H), $3.93(\mathrm{q}, J=7.0 \mathrm{~Hz}, 2 \mathrm{H}), 2.34(\mathrm{q}, J=7.7 \mathrm{~Hz}, 2 \mathrm{H}), 2.20(\mathrm{~s}, 3 \mathrm{H}), 0.99$ (t, $J=7.7$ $\mathrm{Hz}, 3 \mathrm{H}), 0.90$ (t, $J=7.0 \mathrm{~Hz}, 3 \mathrm{H}) ;{ }^{13} \mathrm{C} \mathrm{NMR}\left(176 \mathrm{MHz}, \mathrm{CDCl}_{3}\right) \delta 169.7,153.1,141.5$, 138.9, 136.1, 131.2, 130.1, 127.8, 127.0, 117.9, 115.7, 61.0, 26.1, 15.4, 13.7, 12.5; HRMS (EI) m/z: [M] ${ }^{+}$Calcd for $\mathrm{C}_{18} \mathrm{H}_{20} \mathrm{O}_{3}$ 284.1413; Found 284.1399. 
<smiles>CCOC(=O)c1c(O)c(O)cc(CC)c1-c1ccccc1</smiles>

Ethyl 3,6-diethyl-4-hydroxy-[1,1'-biphenyl]-2-carboxylate (71).

5-Ethyl-3-hydroxy-6-phenyl-2H-pyran-2-one $(43.2 \mathrm{mg}, 0.2 \mathrm{mmol}, 1 \mathrm{eq})$ and ethyl 3-nitropent-2-enoate $(69.2 \mathrm{mg}, 0.4 \mathrm{mmol}, 2 \mathrm{eq})$ were subjected to the general procedure for $3 \mathrm{~h}$. Purification by FCC (15:1 hexanes:EtOAc) yielded 71 as a yellow solid (26.3 mg, 44\%).

Data for 71: Rf: 0.33 (5:1 hexanes:EtOAc); mp: $94-97^{\circ} \mathrm{C}$; IR (thin film) 3399, 2969, 2935, 2873, 1700, 1598, 1443, $1309 \mathrm{~cm}^{-1} ;{ }^{1} \mathrm{H}$ NMR (700 MHz, $\left.\mathrm{CDCl}_{3}\right) \delta 7.33$ (t, $J=$ $7.0 \mathrm{~Hz}, 2 \mathrm{H}), 7.31-7.30(\mathrm{~m}, 1 \mathrm{H}), 7.23-7.21(\mathrm{~m}, 2 \mathrm{H}), 6.75(\mathrm{~s}, 1 \mathrm{H}), 5.08(\mathrm{~s}, 1 \mathrm{H})$, $3.92(\mathrm{q}, J=7.0 \mathrm{~Hz}, 2 \mathrm{H}), 2.61(\mathrm{q}, J=7.7 \mathrm{~Hz}, 2 \mathrm{H}), 2.35$ (q, $J=7.7 \mathrm{~Hz}, 2 \mathrm{H}), 1.22$ (t, $J$ $=7.7 \mathrm{~Hz}, 3 \mathrm{H}), 1.00(\mathrm{t}, J=7.0 \mathrm{~Hz}, 3 \mathrm{H}), 0.91(\mathrm{t}, J=7.7 \mathrm{~Hz}, 3 \mathrm{H}) ;{ }^{13} \mathrm{C}$ NMR $(176$ $\left.\mathrm{MHz}, \mathrm{CDCl}_{3}\right) \delta 169.6,152.8,141.5,138.9,135.9,131.2,130.1,127.7,127.0,124.2$, 116.0, 60.8, 26.1, 21.1, 15.3, 14.5, 13.7; HRMS (EI) m/z: [M] ${ }^{+}$Calcd for $\mathrm{C}_{19} \mathrm{H}_{22} \mathrm{O}_{3}$ 298.1569; Found 298.1583.<smiles>CCc1cc(O)c(CC(C)C)c(CC)c1-c1ccccc1</smiles>

\section{2,6-Diethyl-3-isobutyl-[1,1'-biphenyl]-4-ol (72).}

5-Ethyl-3-hydroxy-4-isobutyl-6-phenyl-2H-pyran- 2-one (27.2 mg, $0.1 \mathrm{mmol}, 1 \mathrm{eq})$ and 1-nitrobut-1-ene $(30.3 \mathrm{mg}, 0.3 \mathrm{mmol}, 3 \mathrm{eq})$ were subjected to the general procedure at $130{ }^{\circ} \mathrm{C}$ for $24 \mathrm{~h}$. Purification by FCC (50:1 hexanes:EtOAc) yielded 72 as a yellow solid (13.3 $\mathrm{mg}, 47 \%)$.

Data for 72: $\mathrm{R}_{\mathrm{f}}$ 0.48 (5:1 hexanes:EtOAc); mp: $55-58{ }^{\circ} \mathrm{C}$; IR (thin film) 3424, 2960, 2931, 2869, 1600, $1463 \mathrm{~cm}^{-1}$; ${ }^{1} \mathrm{H}$ NMR $\left(700 \mathrm{MHz}, \mathrm{CDCl}_{3}\right) \delta 7.39(\mathrm{t}, J=7.7 \mathrm{~Hz}, 2 \mathrm{H})$, $7.33(\mathrm{t}, J=7.7 \mathrm{~Hz}, 1 \mathrm{H}), 7.17(\mathrm{dd}, J=7.7,1.4 \mathrm{~Hz}, 2 \mathrm{H}), 6.59(\mathrm{~s}, 1 \mathrm{H}), 4.61(\mathrm{~s}, 1 \mathrm{H})$, $2.56(\mathrm{~d}, J=7.0 \mathrm{~Hz}, 2 \mathrm{H}), 2.37$ (q, $J=7.7 \mathrm{~Hz}, 2 \mathrm{H}), 2.20$ (q, $J=7.7 \mathrm{~Hz}, 2 \mathrm{H}), 1.97$ (sept, $J=7.0 \mathrm{~Hz}, 1 \mathrm{H}), 1.01-0.99(\mathrm{~m}, 9 \mathrm{H}), 0.87(\mathrm{t}, J=7.7 \mathrm{~Hz}, 3 \mathrm{H}) ;{ }^{13} \mathrm{C}$ NMR $(176$ $\left.\mathrm{MHz}, \mathrm{CDCl}_{3}\right) \delta 153.2,142.4,141.2,141.1,134.3,130.3,127.9,126.4,122.9,112.4$, 34.9, 29.5, 26.7, 23.6, 22.8, 15.3, 15.2; HRMS (EI) m/z: [M] $]^{+}$Calcd for $\mathrm{C}_{20} \mathrm{H}_{26} \mathrm{O}$ 282.1984; Found 282.1990. 
<smiles>CCc1cc(-c2ccccc2)c(CC)c(O)c1CC(C)C</smiles>

\section{2,5-Diethyl-3-isobutyl-[1,1'-biphenyl]-4-ol (73).}

5-Ethyl-3-hydroxy-4-isobutyl-6-phenyl-2H-pyran- 2-one (27.2 mg, $0.1 \mathrm{mmol}, 1 \mathrm{eq})$ and 2-nitrobut-1-ene $(30.3 \mathrm{mg}, 0.3 \mathrm{mmol}, 3 \mathrm{eq})$ were subjected to the general procedure at $130{ }^{\circ} \mathrm{C}$. Purification by FCC (50:1 hexanes:EtOAc) yielded 73 as a yellow oil (18.4 mg, 65\%).

Data for 73: Rf: 0.72 (5:1 hexanes:EtOAc); IR (thin film) 3594, 2961, 2932, 2869, 1458, $1196 \mathrm{~cm}^{-1}$; ${ }^{1} \mathrm{H} \mathrm{NMR}\left(700 \mathrm{MHz}, \mathrm{CDCl}_{3}\right) \delta 7.38(\mathrm{t}, J=7.0 \mathrm{~Hz}, 2 \mathrm{H}), 7.32(\mathrm{tt}, J=$ 7.0, $1.4 \mathrm{~Hz}, 1 \mathrm{H}), 7.30(\mathrm{dd}, J=7.0,1.4 \mathrm{~Hz}, 2 \mathrm{H}), 6.84(\mathrm{~s}, 1 \mathrm{H}), 4.67(\mathrm{~s}, 1 \mathrm{H}), 2.64-$ $2.56(\mathrm{~m}, 6 \mathrm{H}), 1.97$ (sept, $J=7.0 \mathrm{~Hz}, 1 \mathrm{H}), 1.26(\mathrm{t}, J=7.7 \mathrm{~Hz}, 3 \mathrm{H}), 1.02(\mathrm{~d}, J=7.0$ $\mathrm{Hz}, 6 \mathrm{H}), 0.91(\mathrm{t}, J=7.7 \mathrm{~Hz}, 3 \mathrm{H}) ;{ }^{13} \mathrm{C} \mathrm{NMR}\left(176 \mathrm{MHz}, \mathrm{CDCl}_{3}\right) \delta 151.2,143.2,139.3$, 134.8, 129.7, 128.2, 127.8, 126.3, 125.8, 125.2, 35.3, 29.5, 22.9, 22.9, 15.5, 13.7; HRMS (EI) m/z: [M] ${ }^{+}$Calcd for $\mathrm{C}_{20} \mathrm{H}_{26} \mathrm{O}$ 282.1984; Found 282.1974.<smiles>CCc1c(-c2ccccc2)cc(Br)c(O)c1CC(C)C</smiles>

\section{5-Benzyl-2-ethyl-3-isobutyl-[1,1'-biphenyl]-4-ol (74).}

5-Ethyl-3-hydroxy-4-isobutyl-6-phenyl-2H-pyran- 2-one (27.2 mg, $0.1 \mathrm{mmol}, 1 \mathrm{eq})$ and (3-nitroallyl)benzene $(21.9 \mathrm{mg}, 0.3 \mathrm{mmol}, 3 \mathrm{eq})$ were subjected to the general procedure. Purification by FCC (50:1 hexanes:EtOAc) yielded 74 as a yellow oil (21.9 mg, 64\%).

Data for 74: Rf: 0.75 (6:1 hexanes:EtOAc); IR (thin film) 3548, 2957, 2929, 2868, 1602, $1461 \mathrm{~cm}^{-1} ;{ }^{1} \mathrm{H}$ NMR $\left(700 \mathrm{MHz}, \mathrm{CDCl}_{3}\right) \delta 7.28(\mathrm{t}, J=7.0 \mathrm{~Hz}, 2 \mathrm{H}), 7.33-7.30$ $(\mathrm{m}, 5 \mathrm{H}), 7.26(\mathrm{~d}, J=7.0 \mathrm{~Hz}, 2 \mathrm{H}), 7.22(\mathrm{t}, J=7.0 \mathrm{~Hz}, 1 \mathrm{H}), 6.86(\mathrm{~s}, 1 \mathrm{H}), 4.62(\mathrm{~s}, 1$ H), 3.99 (s, $2 \mathrm{H}), 2.61-2.57(\mathrm{~m}, 4 \mathrm{H}), 1.95$ (sept, $J=7.0 \mathrm{~Hz}, 1 \mathrm{H}), 0.98$ (d, $J=7.0$ $\mathrm{Hz}, 6 \mathrm{H}), 0.92(\mathrm{t}, J=7.0 \mathrm{~Hz}, 3 \mathrm{H}) ;{ }^{13} \mathrm{C} \mathrm{NMR}\left(176 \mathrm{MHz}, \mathrm{CDCl}_{3}\right) \delta 151.7,143.0,140.2$, 139.7, 135.0, 130.2, 129.7, 128.8, 128.7, 127.8, 126.5, 126.3, 126.1, 123.3, 37.0, 35.2, 29.5, 22.9, 22.8, 15.5; HRMS (EI) m/z: [M] Calcd for $\mathrm{C}_{25} \mathrm{H}_{28} \mathrm{O}$ 344.2140; Found 344.2149 .<smiles>CCOC(=O)c1c(C)c(O)c(C)c(CC)c1-c1ccccc1</smiles> 
Ethyl 6-ethyl-4-hydroxy-3,5-dimethyl-[1,1'-biphenyl]-2-carboxylate (75).

5-Ethyl-3-hydroxy-4-methyl-6-phenyl-2H-pyran- 2-one (46 mg, $0.2 \mathrm{mmol}, 2 \mathrm{eq}$ ) and ethyl 3-nitrobut-2-enoate $(15.9 \mathrm{mg}, 0.1 \mathrm{mmol}, 1 \mathrm{eq})$ were subjected to the general procedure for 3 days. Purification by FCC (10:1 hexanes:EtOAc) yielded $\mathbf{7 5}$ as a yellow oil (15.8 mg, 53\%).

Data for 75: Rf: 0.36 (7:1 hexanes:EtOAc); IR (thin film) 3455, 2971, 2932, 1703, 1443, 1289, $1202 \mathrm{~cm}^{-1} ;{ }^{1} \mathrm{H} \mathrm{NMR}\left(700 \mathrm{MHz}, \mathrm{CDCl}_{3}\right) \delta 7.33(\mathrm{tt}, J=6.3,1.4 \mathrm{~Hz}, 2 \mathrm{H})$, $7.30(\mathrm{tt}, J=6.3,1.4 \mathrm{~Hz}, 1 \mathrm{H}), 7.21(\mathrm{dt}, J=6.3,1.4 \mathrm{~Hz}, 2 \mathrm{H}), 4.81(\mathrm{~s}, 1 \mathrm{H}), 3.88(\mathrm{q}, J=$ $7.0 \mathrm{~Hz}, 2 \mathrm{H}), 2.43$ (q, $J=7.7 \mathrm{~Hz}, 2 \mathrm{H}), 2.27(\mathrm{~s}, 3 \mathrm{H}), 2.21(\mathrm{~s}, 3 \mathrm{H}), 0.93$ (t, $J=7.7 \mathrm{~Hz}$, $3 \mathrm{H}), 0.89$ (t, $J=7.0 \mathrm{~Hz}, 3 \mathrm{H}) ;{ }^{13} \mathrm{C} \mathrm{NMR}\left(176 \mathrm{MHz}, \mathrm{CDCl}_{3}\right) \delta 169.5,151.5,139.8$, $139.8,133.6,131.4,130.2,127.6,126.9,122.7,116.8,60.7,23.6,14.6,13.7,13.0$, 12.0; HRMS (EI) m/z: [M] $]^{+}$Calcd for $\mathrm{C}_{19} \mathrm{H}_{22} \mathrm{O}_{3} 298.1569$; Found 298.1574.<smiles>CCOC(=O)c1c(CC)c(O)c(C)c(CC)c1-c1ccccc1</smiles>

Ethyl 3,6-diethyl-4-hydroxy-5-methyl-[1,1'-biphenyl]-2-carboxylate (76).

5-Ethyl-3-hydroxy-4-methyl-6-phenyl-2H-pyran- 2-one (23 mg, $0.1 \mathrm{mmol}, 1 \mathrm{eq})$ and ethyl 3-nitropent-2-enoate $(86.5 \mathrm{mg}, 0.5 \mathrm{mmol}, 5 \mathrm{eq})$ were subjected to the general procedure for 2 days. Purification by FCC (20:1 hexanes:EtOAc) yielded 76 as a yellow oil (20.6 mg, 66\%).

Data for 76: Rf: 0.45 (5:1 hexanes:EtOAc); IR (thin film) 3480, 2970, 2934, 1705, 1285, $1202 \mathrm{~cm}^{-1} ;{ }^{1} \mathrm{H}$ NMR $\left(700 \mathrm{MHz}, \mathrm{CDCl}_{3}\right) \delta 7.35(\mathrm{t}, J=7.0 \mathrm{~Hz}, 2 \mathrm{H}), 7.30$ (t, $J=$ $7.0 \mathrm{~Hz}, 1 \mathrm{H}), 7.24(\mathrm{~d}, J=7.0 \mathrm{~Hz}, 2 \mathrm{H}), 5.07$ (s, $1 \mathrm{H}), 3.89$ (q, J=7.0 Hz, $2 \mathrm{H}), 2.61$ (q, $J=7.0 \mathrm{~Hz}, 2 \mathrm{H}), 2.43(\mathrm{q}, J=7.0 \mathrm{~Hz}, 2 \mathrm{H}), 2.28(\mathrm{~s}, 3 \mathrm{H}), 1.23(\mathrm{t}, J=7.0 \mathrm{~Hz}, 3 \mathrm{H})$, $0.95(\mathrm{t}, J=7.0 \mathrm{~Hz}, 3 \mathrm{H}), 0.91(\mathrm{t}, J=7.0 \mathrm{~Hz}, 3 \mathrm{H}) ;{ }^{13} \mathrm{C} \mathrm{NMR}\left(176 \mathrm{MHz}, \mathrm{CDCl}_{3}\right) \delta$ $169.7,151.2,139.8,133.2,131.4,130.3,127.6,126.9,123.3,123.0,60.7,23.7,21.6$, 14.6, 14.5, 13.7, 12.0; HRMS (ESI) m/z: $[\mathrm{M}+\mathrm{H}]^{+}$Calcd for $\mathrm{C}_{20} \mathrm{H}_{25} \mathrm{O}_{3} 313.1804$; Found 313.1802.<smiles>CCOc1c(O)c(CC(C)C)c(CC)c(CC)c1-c1ccccc1</smiles>

Ethyl 6-ethyl-4-hydroxy-5-isobutyl-3-methyl-[1,1'-biphenyl]-2-carboxylate (77). 5-Ethyl-3-hydroxy-4-isobutyl-6-phenyl-2H-pyran- 2-one (54.4 mg, $0.2 \mathrm{mmol}, 1 \mathrm{eq})$ and ethyl 3-nitrobut-2-enoate $(63.4 \mathrm{mg}, 0.4 \mathrm{mmol}, 2 \mathrm{eq})$ were subjected to the general procedure for 4 days. Purification by FCC (7:1 hexanes:EtOAc) yielded 77 as a yellow oil (48.1 mg, 71\%). 
Data for 77: Rf: 0.27 (7:1 hexanes:EtOAc); IR (thin film) 3478, 2958, 2932, 2869, 1706, 1430, 1296, $1202 \mathrm{~cm}^{-1}$; ${ }^{1} \mathrm{H} \mathrm{NMR}\left(700 \mathrm{MHz}, \mathrm{CDCl}_{3}\right) \delta 7.33(\mathrm{t}, J=7.0 \mathrm{~Hz}, 2 \mathrm{H})$, $7.30(\mathrm{t}, J=7.0 \mathrm{~Hz}, 1 \mathrm{H}), 7.23(\mathrm{~d}, J=7.0 \mathrm{~Hz}, 2 \mathrm{H}), 4.82(\mathrm{~s}, 1 \mathrm{H}), 3.89$ (q, $J=7.0 \mathrm{~Hz}, 2$ H), $2.58(\mathrm{~d}, J=7.7 \mathrm{~Hz}, 2 \mathrm{H}), 2.45(\mathrm{q}, J=7.7 \mathrm{~Hz}, 2 \mathrm{H}), 2.20(\mathrm{~s}, 3 \mathrm{H}), 1.95$ (sept, $J=$ $7.0 \mathrm{~Hz}, 1 \mathrm{H}), 0.99$ (d, $J=7.0 \mathrm{~Hz}, 6 \mathrm{H}), 0.89$ (t, $J=7.0 \mathrm{~Hz}, 3 \mathrm{H}), 0.86(\mathrm{t}, J=7.7 \mathrm{~Hz}, 3$ $\mathrm{H}) ;{ }^{13} \mathrm{C}$ NMR $\left(176 \mathrm{MHz}, \mathrm{CDCl}_{3}\right) \delta 167.7,151.6,140.2,139.8,133.9,131.5,130.3$, 127.6, 126.9, 126.6, 116.9, 60.8, 35.3, 29.4, 23.1, 22.8, 15.3, 13.7, 13.0; HRMS (ESI) $\mathrm{m} / \mathrm{z}:[\mathrm{M}+\mathrm{H}]^{+}$Calcd for $\mathrm{C}_{22} \mathrm{H}_{29} \mathrm{O}_{3} 341.2117$; Found 341.2127.<smiles>CCOC(=O)c1c(CC)c(O)c(CC(C)C)c(CC)c1-c1ccccc1</smiles>

Ethyl 3,6-diethyl-4-hydroxy-5-isobutyl-[1,1'-biphenyl]-2-carboxylate (78).

5-Ethyl-3-hydroxy-4-isobutyl-6-phenyl-2H-pyran- 2-one (27.2 mg, $0.1 \mathrm{mmol}, 1 \mathrm{eq})$ and ethyl 3-nitropent-2-enoate (34.6 $\mathrm{mg}, 0.2 \mathrm{mmol}, 2 \mathrm{eq})$ were subjected to the general procedure at $180{ }^{\circ} \mathrm{C}$ for 3 days. Purification by FCC (15:1 hexanes:EtOAc) yielded 78 as a yellow oil (11.3 $\mathrm{mg}, 32 \%)$.

Data for 78: Rf: 0.45 (5:1 hexanes:EtOAc); IR (thin film) 3494, 2962, 2871, 1707, $1465 \mathrm{~cm}^{-1} ;{ }_{1}^{1} \mathrm{H}$ NMR $\left(700 \mathrm{MHz}, \mathrm{CDCl}_{3}\right) \delta 7.33(\mathrm{t}, J=7.0 \mathrm{~Hz}, 2 \mathrm{H}), 7.29(\mathrm{t}, J=7.0 \mathrm{~Hz}$, $1 \mathrm{H}), 7.24(\mathrm{~d}, J=7.0 \mathrm{~Hz}, 2 \mathrm{H}), 4.79(\mathrm{~s}, 1 \mathrm{H}), 3.87$ (q, $J=7.0 \mathrm{~Hz}, 2 \mathrm{H}), 2.61-2.57$ (m, $4 \mathrm{H}), 2.45$ (q, $J=7.7 \mathrm{~Hz}, 2 \mathrm{H}), 1.95$ (sept, $J=7.0 \mathrm{~Hz}, 1 \mathrm{H}$ ), 1.22 (t, $J=7.7 \mathrm{~Hz}, 3 \mathrm{H}$ ), $1.00(\mathrm{~d}, J=7.0 \mathrm{~Hz}, 6 \mathrm{H}), 0.90(\mathrm{t}, J=7.0 \mathrm{~Hz}, 3 \mathrm{H}), 0.86(\mathrm{t}, J=7.7 \mathrm{~Hz}, 3 \mathrm{H}) ;{ }^{13} \mathrm{C} \mathrm{NMR}$ $\left(176 \mathrm{MHz}, \mathrm{CDCl}_{3}\right) \delta 169.6,151.2,140.2,139.8,133.6,131.5,130.4,127.5,126.8$, 126.6, 123.1, 60.6, 35.4, 29.5, 23.1, 22.8, 21.6, 15.2, 14.4, 13.7; HRMS (EI) m/z: $[\mathrm{M}]^{+}$Calcd for $\mathrm{C}_{23} \mathrm{H}_{30} \mathrm{O}_{3} 354.2195$; Found 354.2180.<smiles>CCOc1c(O)c(CC(C)C)c(C)c(-c2ccccc2)c1OCC</smiles>

Ethyl 3-ethyl-4-hydroxy-5-isobutyl-6-methyl-[1,1'-biphenyl]-2-carboxylate (79). 3-Hydroxy-4-isobutyl-5-methyl-6-phenyl-2H-pyran- 2-one (258 mg, $0.1 \mathrm{mmol}, 1 \mathrm{eq})$ and ethyl 3-nitropent-2-enoate (346 mg, $0.2 \mathrm{mmol}, 2 \mathrm{eq}$ ) were subjected to the general procedure at $180{ }^{\circ} \mathrm{C}$. Purification by FCC (20:1 hexanes:EtOAc) yielded 79 as a yellow oil (249.3 mg, 70\%).

Data for 79: Rf: 0.48 (5:1 hexanes:EtOAc); mp: $84-89^{\circ} \mathrm{C}$; IR (thin film) 3494, 2956, 2926, 2869, 1707, 1464, 1295, $1198 \mathrm{~cm}^{-1} ;{ }^{1} \mathrm{H}$ NMR (700 MHz, $\left.\mathrm{CDCl}_{3}\right) \delta 7.35$ (t, $J=$ $7.0 \mathrm{~Hz}, 2 \mathrm{H}), 7.30$ (t, J=7.0 Hz, $1 \mathrm{H}), 7.22$ (d, $J=7.0 \mathrm{~Hz}, 2 \mathrm{H}), 5.03$ (s, $1 \mathrm{H}), 3.92$ (q, 
$J=7.0 \mathrm{~Hz}, 2 \mathrm{H}), 2.63$ (q, $J=7.0 \mathrm{~Hz}, 2 \mathrm{H}), 2.60(\mathrm{~d}, J=7.7 \mathrm{~Hz}, 2 \mathrm{H}), 2.03(\mathrm{~s}, 3 \mathrm{H})$, 1.94 (sept, $J=7.0 \mathrm{~Hz}, 1 \mathrm{H}), 1.24$ (t, $J=7.0 \mathrm{~Hz}, 3 \mathrm{H}), 1.01$ (d, $J=7.0 \mathrm{~Hz}, 6 \mathrm{H}), 0.91$ (t, $J=7.0 \mathrm{~Hz}, 3 \mathrm{H}) ;{ }^{13} \mathrm{C} \mathrm{NMR}\left(176 \mathrm{MHz}, \mathrm{CDCl}_{3}\right) \delta 169.8,151.0,140.4,133.9,133.2$, 131.9, 130.1, 127.8, 127.6, 126.8, 123.4, 60.7, 36.0, 28.9, 22.8, 21.6, 17.4, 14.5, 13.7; HRMS (ESI) m/z: [M+ H] $]^{+}$Calcd for $\mathrm{C}_{22} \mathrm{H}_{29} \mathrm{O}_{3} 341.2117$; Found 341.2121. 


\section{Syntheses of Substituted Benzenes}

\section{General Procedure for Triflate Formation}<smiles>[R]c1c([R])c([R])c(O)c([R])c1[R]</smiles>

To a solution of phenol $(1 \mathrm{eq})$ in $\operatorname{DCM}(0.2 \mathrm{M})$ was added pyridine $(1.5 \mathrm{eq})$ at $0{ }^{\circ} \mathrm{C}$. After stirring for $5 \mathrm{mins}$, triflic anhydride (1.3 eq) was added dropwise. The mixture was stirred at $0{ }^{\circ} \mathrm{C}$ for 30 mins, then $\mathrm{rt}$ for $2 \mathrm{~h}$. The mixture was quenched with $2 \mathrm{~N}$ $\mathrm{HCl}$ and extracted with DCM (3 X). The combined organic layers were dried over $\mathrm{Na}_{2} \mathrm{SO}_{4}$, filtered and concentrated. The residue was purified by FCC.

\section{General Procedure for Stille Couplings:}<smiles>[R]c1c([R])c([R])c([OH+])c([R5])c1[R7]</smiles>

To a thick-walled reaction vessel were added the triflate (1 eq), $\mathrm{Pd}\left(\mathrm{PPh}_{3}\right)_{4}(10 \mathrm{~mol} \%)$, and DMF $(0.1 \mathrm{M})$ under Ar. The stannane (1.3 eq) was added dropwise, and the tube was sealed and heated to $90{ }^{\circ} \mathrm{C}$ for $16 \mathrm{~h}$ unless otherwise noted. The reaction mixture was cooled to rt, EtOAc was added and the organic layer was washed with brine. The organic layer was dried over $\mathrm{Na}_{2} \mathrm{SO}_{4}$, filtered and concentrated. The residue was purified by FCC.

\section{General Procedure for Suzuki Couplings:}<smiles>[R]Cc1c([R])c([R])c([OH+])c([R])c1[R]</smiles><smiles>[R]B(CC)C(=C)CCO</smiles>

boronic ester or acid<smiles>[R]c1c([R])c([R])c(C(=C)CCO)c([R])c1[R]</smiles>

To a thick-walled reaction vessel were added the triflate $(1 \mathrm{eq})$, boronic ester or acid (1.5 eq), $\mathrm{Pd}\left(\mathrm{PPh}_{3}\right)_{4}(10 \mathrm{~mol} \%), \mathrm{K}_{2} \mathrm{CO}_{3}$ (3 eq) and 1, 4-dioxane/ water mixture (4:1, $0.1 \mathrm{M})$. The headspace of the vessel was evacuated by brief exposure to vacuum, and the vessel was back-filled with Ar. The vessel was evacuated and back-filled with Ar two additional times. The tube was sealed and heated to $110^{\circ} \mathrm{C}$ for $16 \mathrm{~h}$ unless otherwise noted. The reaction was cooled to $\mathrm{rt}$, and solvent was removed under vacuum. The crude reaction mixture was dissolved in EtOAc, filtered through celite, dried over $\mathrm{Na}_{2} \mathrm{SO}_{4}$, filtered, and concentrated. The residue was purified by FCC. 
<smiles>C=CCc1c(CC)ccc(C)c1CC</smiles>

\section{2-Allyl-3-ethyl-1-isobutyl-4-methylbenzene (80).}

2-Ethyl-6-isobutyl-3-methylphenol (55) (80 $\mathrm{mg}, 0.417 \mathrm{mmol}, 1 \mathrm{eq})$ was subjected to the general triflate formation procedure. Purification by FCC (100\% Hexane) yielded 2-Ethyl-6-isobutyl-3-methylphenyl trifluoromethanesulfonate (103.5 mg, 77\%).

2-Ethyl-6-isobutyl-3-methylphenyl trifluoromethanesulfonate $(21 \mathrm{mg}, 0.07 \mathrm{mmol}, 1$ eq) and allyl tributylstannane $(28 \mathrm{mg}, 0.085 \mathrm{mmol}, 1.2 \mathrm{eq})$ were subjected to the general Stille coupling procedure with a reaction time of 6 days. Purification by FCC (100\% hexanes) yielded $\mathbf{8 0}$ as a colorless oil ( $8.8 \mathrm{mg}, 63 \%)$.

Data for 80: Rf: 0.59 (Pure hexanes); IR (thin film) 3079, 2956, 2928, 2868, 1636, $1461 \mathrm{~cm}^{-1} ;{ }^{1} \mathrm{H}$ NMR $\left(700 \mathrm{MHz}, \mathrm{CDCl}_{3}\right) \delta 6.96(\mathrm{~d}, J=7.7 \mathrm{~Hz}, 1 \mathrm{H}), 6.89(\mathrm{~d}, J=7.7$ $\mathrm{Hz}, 1 \mathrm{H}), 5.95$ (ddt, $J=16.8,10.5,5.6 \mathrm{~Hz}, 1 \mathrm{H}), 4.99(\mathrm{dd}, J=9.8,1.4 \mathrm{~Hz}, 1 \mathrm{H}), 4.82$ $(\mathrm{dd}, J=16.8,1.4 \mathrm{~Hz}, 1 \mathrm{H}), 3.43(\mathrm{dt}, J=5.6,2.1 \mathrm{~Hz}, 2 \mathrm{H}), 2.64(\mathrm{q}, J=7.7 \mathrm{~Hz}, 2 \mathrm{H})$, $2.43(\mathrm{~d}, J=7.7 \mathrm{~Hz}, 2 \mathrm{H}), 2.31(\mathrm{~s}, 3 \mathrm{H}), 1.85$ (sept, $J=7.0 \mathrm{~Hz}, 1 \mathrm{H}), 1.12$ (t, $J=7.7 \mathrm{~Hz}$, $3 \mathrm{H}), 1.92(\mathrm{~d}, J=7.0 \mathrm{~Hz}, 6 \mathrm{H}) ;{ }^{13} \mathrm{C} \mathrm{NMR}\left(176 \mathrm{MHz}, \mathrm{CDCl}_{3}\right) \delta 141.3,138.1,137.6$, 135.1, 133.6, 128.1, 127.8, 114.9, 42.6, 32.7, 29.5, 22.7, 22.7, 19.7, 14.3; HRMS (EI) $\mathrm{m} / \mathrm{z}:[\mathrm{M}]^{+}$Calcd for $\mathrm{C}_{16} \mathrm{H}_{24} 216.1878$; Found 216.1884.<smiles>C=C(CCO)c1c(CC)ccc(C)c1CC</smiles>

\section{3-(2-Ethyl-6-isobutyl-3-methylphenyl)but-3-en-1-ol (81).}

2-Ethyl-6-isobutyl-3-methylphenyl trifluoromethanesulfonate $(21 \mathrm{mg}, 0.07 \mathrm{mmol}, 1$ eq) and 3-(4,4,5,5-tetramethyl-1,3,2-dioxaborolan-2-yl)but-3-en-1-ol (18.3 mg, 0.09 mmol, $1.3 \mathrm{eq})$ were subjected to the general Suzuki coupling procedure with a reaction time of 4 days. Purification by FCC (5:1 hexanes:EtOAc) yielded $\mathbf{8 1}$ as a colorless oil (8.6 mg, 54\%).

Data for 81: Rf: 0.38 (5:1 hexanes:EtOAc); IR (thin film) 3337, 2955, 2932, 2869, 1637, $1466 \mathrm{~cm}^{-1} ;{ }^{1} \mathrm{H}$ NMR $\left(700 \mathrm{MHz}, \mathrm{CDCl}_{3}\right) \delta 7.02(\mathrm{~d}, J=7.7 \mathrm{~Hz}, 1 \mathrm{H}), 6.95(\mathrm{~d}, J=$ $7.7 \mathrm{~Hz}, 1 \mathrm{H}), 5.35(\mathrm{dd}, J=2.8,1.4 \mathrm{~Hz}, 1 \mathrm{H}), 4.94(\mathrm{~d}, J=1.4 \mathrm{~Hz}, 1 \mathrm{H}), 3.86$ (app q, $J$ $=6.3 \mathrm{~Hz}, 2 \mathrm{H}), 2.68-2.62(\mathrm{~m}, 1 \mathrm{H}), 2.52-2.49(\mathrm{~m}, 3 \mathrm{H}), 2.43(\mathrm{dd}, J=14.0,7.0 \mathrm{~Hz}$, $1 \mathrm{H}), 2.33(\mathrm{dd}, J=14.0,7.0 \mathrm{~Hz}, 1 \mathrm{H}), 2.32(\mathrm{~s}, 3 \mathrm{H}), 1.88$ (sept, $J=7.0 \mathrm{~Hz}, 1 \mathrm{H}), 1.41$ $(\mathrm{s}, 1 \mathrm{H}), 1.10(\mathrm{t}, J=7.0 \mathrm{~Hz}, 3 \mathrm{H}), 0.92(\mathrm{~d}, J=7.0 \mathrm{~Hz}, 3 \mathrm{H}), 0.87$ (d, $J=7.0 \mathrm{~Hz}, 3 \mathrm{H})$; ${ }^{13} \mathrm{C} \mathrm{NMR}\left(176 \mathrm{MHz}, \mathrm{CDCl}_{3}\right) \delta 145.1,142.0,139.4,136.2,133.5,128.8,126.8,115.0$, 60.5, 42.1 41.3, 29.8, 23.8, 22.7, 22.6, 19.4, 15.1; HRMS (ESI) m/z: [M+ Na $]^{+}$Calcd 
for $\mathrm{C}_{17} \mathrm{H}_{26} \mathrm{ONa} 269.1881$; Found 269.1875.<smiles>C=CCc1c(C)ccc(C(=O)OCC)c1C</smiles>

\section{Ethyl 3-allyl-2,4-dimethylbenzoate (82).}

Ethyl 3-hydroxy-2,4-dimethylbenzoate (56) (100 mg, $0.515 \mathrm{mmol}, 1 \mathrm{eq})$ was subjected to the general triflate formation procedure. Purification by FCC (50:1 to 10:1 hexanes:EtOAc) yielded Ethyl 2,4-dimethyl-3-(((trifluoromethyl)sulfonyl)oxy) benzoate (113.4 mg, 67\%).

Ethyl 2,4-dimethyl-3-(((trifluoromethyl)sulfonyl)oxy)benzoate (32.6 mg, $0.1 \mathrm{mmol}, 1$ eq) and allyl tributylstannane (40 $\mathrm{mg}, 0.12 \mathrm{mmol}, 1.2 \mathrm{eq})$ were subjected to the general Stille coupling procedure. Purification by FCC (50:1 hexanes:EtOAc) yielded $\mathbf{8 2}$ as a yellow oil (19.2 $\mathrm{mg}, 88 \%)$.

Data for 82: Rf: 0.74 (10:1 hexanes:EtOAc); IR (thin film) 3082, 2979, 2933, 1717, $1264 \mathrm{~cm}^{-1} ;{ }^{1} \mathrm{H}$ NMR $\left(700 \mathrm{MHz}, \mathrm{CDCl}_{3}\right) \delta 7.57(\mathrm{~d}, J=7.7 \mathrm{~Hz}, 1 \mathrm{H}), 7.05(\mathrm{~d}, J=7.7$ $\mathrm{Hz}, 1 \mathrm{H}), 5.91$ (ddt, $J=16.8,9.8,4.9 \mathrm{~Hz}, 1 \mathrm{H}), 5.01$ (dq, $J=10.5,2.1 \mathrm{~Hz}, 1 \mathrm{H}), 4.82$ $(\mathrm{dq}, J=17.5,2.1 \mathrm{~Hz}, 1 \mathrm{H}), 4.35(\mathrm{q}, J=7.0 \mathrm{~Hz}, 2 \mathrm{H}), 3.45(\mathrm{dt}, J=5.6,2.1 \mathrm{~Hz}, 2 \mathrm{H})$, $2.48(\mathrm{~s}, 3 \mathrm{H}), 2.33(\mathrm{~s}, 3 \mathrm{H}), 1.38(\mathrm{t}, J=7.7 \mathrm{~Hz}, 3 \mathrm{H}) ;{ }^{13} \mathrm{C} \mathrm{NMR}\left(176 \mathrm{MHz}, \mathrm{CDCl}_{3}\right) \delta$ 168.9, 140.8, 137.6, 137.4, 134.8, 129.7, 127.8, 127.5, 115.2, 60.7, 33.5, 20.5, 16.5, 14.4; HRMS (ESI) m/z: [M + H] $]^{+}$Calcd for $\mathrm{C}_{14} \mathrm{H}_{19} \mathrm{O}_{2} 219.1385$; Found 219.1384.<smiles>C=C(CCO)c1c(C)ccc(C(=O)OCC)c1C</smiles>

\section{Ethyl 3-(4-hydroxybut-1-en-2-yl)-2,4-dimethylbenzoate (83).}

Ethyl 2,4-dimethyl-3-(((trifluoromethyl)sulfonyl)oxy)benzoate $(32.6 \mathrm{mg}, 0.1 \mathrm{mmol}, 1$ eq) and 3-(4,4,5,5-tetramethyl-1,3,2-dioxaborolan-2-yl)but-3-en-1-ol (26 mg, 0.13 mmol, $1.3 \mathrm{eq})$ were subjected to the general Suzuki coupling procedure. Purification by FCC (5:1 hexanes:EtOAc) yielded $\mathbf{8 3}$ as a yellow oil (20 mg, 81\%)

Data for 83: Rf: 0.34 (3:1 hexanes:EtOAc); IR (thin film) 3419, 2980, 2931, 1716, $1591 \mathrm{~cm}^{-1} ;{ }^{1} \mathrm{H} \mathrm{NMR}\left(700 \mathrm{MHz}, \mathrm{CDCl}_{3}\right) \delta 7.64(\mathrm{~d}, J=7.7 \mathrm{~Hz}, 1 \mathrm{H}), 7.08(\mathrm{~d}, J=7.7 \mathrm{~Hz}$, $1 \mathrm{H}), 5.41$ (q, $J=1.4 \mathrm{~Hz}, 1 \mathrm{H}), 4.94(\mathrm{~d}, J=1.4 \mathrm{~Hz}, 1 \mathrm{H}), 4.34(\mathrm{q}, J=7.0 \mathrm{~Hz}, 2 \mathrm{H})$, $3.82-3.76(\mathrm{~m}, 2 \mathrm{H}), 2.51-2.49(\mathrm{~m}, 2 \mathrm{H}), 2.47$ (s, $3 \mathrm{H}), 2.29$ (s, $1 \mathrm{H}), 1.57$ (brs, $1 \mathrm{H})$, $1.38(\mathrm{t}, J=7.0 \mathrm{~Hz}, 3 \mathrm{H}) ;{ }^{13} \mathrm{C} \mathrm{NMR}\left(176 \mathrm{MHz}, \mathrm{CDCl}_{3}\right) \delta 168.4,144.9,143.5,139.3$, 136.3, 128.8, 128.6, 127.3, 116.0, 60.7, 60.4, 40.3, 20.7, 17.9, 14.4; HRMS (ESI) m/z: $[\mathrm{M}+\mathrm{H}]^{+}$Calcd for $\mathrm{C}_{15} \mathrm{H}_{21} \mathrm{O}_{3} 249.1491$; Found 249.1497 . 
<smiles>C=CCc1c(C)c(CC)c(-c2ccccc2)c(C(=O)OCC)c1CC</smiles>

Ethyl 4-allyl-3,6-diethyl-5-methyl-[1,1'-biphenyl]-2-carboxylate (84).

Ethyl 3,6-diethyl-4-hydroxy-5-methyl-[1,1'-biphenyl]-2-carboxylate (76) (127 mg, $0.407 \mathrm{mmol}, 1 \mathrm{eq})$ was subjected to the general triflate formation procedure. Purification by FCC (20:1 hexanes:EtOAc) yielded Ethyl 3,6-diethyl-5-methyl -4-((trifluoromethyl)sulfonyl)oxy)-[1,1'-biphenyl]-2-carboxylate (173 mg, 96\%).

Ethyl 3,6-diethyl-5-methyl-4-(((trifluoromethyl)sulfonyl)oxy)-[1,1'-biphenyl]-2carboxylate (44.4 mg, $0.1 \mathrm{mmol}, 1 \mathrm{eq}$ ) and allyl tributylstannane $(43 \mathrm{mg}, 0.13 \mathrm{mmol}$, $1.3 \mathrm{eq})$ were subjected to the general Stille couplings procedure. Purification by FCC (40:1 hexanes:Aceton) yielded $\mathbf{8 4}$ as a colorless oil (32 mg, 95\%).

Data for 84: Rf: 0.57 (10:1 hexanes:Aceton); IR (thin film) 3082, 2974, 2934, 2875, 1726, $1274 \mathrm{~cm}^{-1} ;{ }^{1} \mathrm{H}$ NMR $\left(700 \mathrm{MHz}, \mathrm{CDCl}_{3}\right) \delta 7.34(\mathrm{t}, J=7.0 \mathrm{~Hz}, 2 \mathrm{H}), 7.30(\mathrm{t}, J=$ $7.0 \mathrm{~Hz}, 1 \mathrm{H}), 7.25$ (d, $J=7.0 \mathrm{~Hz}, 2 \mathrm{H}), 5.97$ (ddt, $J=15.4,9.8,4.9 \mathrm{~Hz}, 1 \mathrm{H}), 5.07$ (d, $J=10.5 \mathrm{~Hz}, 1 \mathrm{H}), 4.94(\mathrm{~d}, J=17.5 \mathrm{~Hz}, 1 \mathrm{H}), 3.86(\mathrm{q}, J=7.0 \mathrm{~Hz}, 2 \mathrm{H}), 3.51(\mathrm{~d}, J=$ $3.5 \mathrm{~Hz}, 2 \mathrm{H}), 2.61$ (q, $J=7.0 \mathrm{~Hz}, 2 \mathrm{H}), 2.44$ (q, $J=7.0 \mathrm{~Hz}, 2 \mathrm{H}), 2.31(\mathrm{~s}, 3 \mathrm{H}), 1.20$ (t, $J=7.0 \mathrm{~Hz}, 3 \mathrm{H}), 0.94(\mathrm{t}, J=7.0 \mathrm{~Hz}, 3 \mathrm{H}), 0.90(\mathrm{t}, J=7.0 \mathrm{~Hz}, 3 \mathrm{H}) ;{ }^{13} \mathrm{C}$ NMR $(176$ $\left.\mathrm{MHz}, \mathrm{CDCl}_{3}\right) \delta 170.3,139.9,138.7,137.1,136.7,136.1,135.7,135.6,133.4,129.9$, 127.5, 126.9, 115.5, 60.5, 33.5, 24.4, 23.8, 15.8, 15.7, 14.6, 13.7; HRMS (ESI) m/z: $[\mathrm{M}+\mathrm{H}]^{+}$Calcd for $\mathrm{C}_{23} \mathrm{H}_{29} \mathrm{O}_{2} 337.2168$; Found 337.2164.<smiles>C=C(CCO)c1c(C)c(CC)c(-c2ccccc2)c(C(=O)OCC)c1C(=O)OCC</smiles>

Ethyl 3,6-diethyl-4-(4-hydroxybut-1-en-2-yl)-5-methyl-[1,1'-biphenyl]-2carboxylate (85).

Ethyl 3,6-diethyl-5-methyl-4-(((trifluoromethyl)sulfonyl)oxy)-[1,1'-biphenyl]-2carboxylate $(23 \mathrm{mg}, 0.052 \mathrm{mmol}, 1 \mathrm{eq})$ and (4-hydroxybut-1-en-2-yl)boronic acid (12 $\mathrm{mg}, 0.104 \mathrm{mmol}, 2 \mathrm{eq})$ were subjected to the general Suzuki coupling procedure with microwave heating $\left(110^{\circ} \mathrm{C}\right)$ and reaction time of $20 \mathrm{~h}$. Purification by FCC (5:1 hexanes:EtOAc) yielded $\mathbf{8 5}$ as a yellow oil (11.9 $\mathrm{mg}, 63 \%)$.

Data for 85: Rf: 0.12 (5:1 hexanes:EtOAc); IR (thin film) 3441, 2970, 2934, 2876, $1723 \mathrm{~cm}^{-1} ;{ }^{1} \mathrm{H} \mathrm{NMR}\left(700 \mathrm{MHz}, \mathrm{CDCl}_{3}\right) \delta 7.35(\mathrm{t}, J=7.0 \mathrm{~Hz}, 2 \mathrm{H}), 7.31(\mathrm{tt}, J=7.0$, $1.4 \mathrm{~Hz}, 1 \mathrm{H}), 7.27-7.26$ (m, $2 \mathrm{H}), 7.23$ (dd, $J=7.7,2.1 \mathrm{~Hz}, 1 \mathrm{H}), 5.42$ (d, $J=1.4 \mathrm{~Hz}$, 
$1 \mathrm{H}), 5.05(\mathrm{~d}, J=1.4 \mathrm{~Hz}, 1 \mathrm{H}), 3.89-3.88(\mathrm{~m}, 2 \mathrm{H}), 3.87-3.82(\mathrm{~m}, 2 \mathrm{H}), 2.65-2.60$ (m, $1 \mathrm{H}), 2.58-2.51(\mathrm{~m}, 3 \mathrm{H}), 2.43(\mathrm{q}, J=7.7 \mathrm{~Hz}, 2 \mathrm{H}), 2.29$ (s, $3 \mathrm{H}), 1.49$ (brs, $1 \mathrm{H})$, $1.13(\mathrm{t}, J=7.0 \mathrm{~Hz}, 3 \mathrm{H}), 0.93(\mathrm{t}, J=7.0 \mathrm{~Hz}, 3 \mathrm{H}), 0.89(\mathrm{t}, J=7.0 \mathrm{~Hz}, 3 \mathrm{H}) ;{ }^{13} \mathrm{C} \mathrm{NMR}$ $\left(176 \mathrm{MHz}, \mathrm{CDCl}_{3}\right) \delta 170.1,145.2,142.0,139.7,138.7,137.9,134.6,134.5,133.0$, 129.9 , 129.8, 127.6, 127.6, 127.0, 115.5, 60.5, 60.5, 40.8, 24.5, 23.7, 16.7, 16.6, 14.3, 13.7; HRMS (ESI) m/z: [M + H] $]^{+}$Calcd for $\mathrm{C}_{24} \mathrm{H}_{31} \mathrm{O}_{3}$ 367.2273; Found 367.2267. 


\section{References Cited}

${ }^{1}$ M. Grigalunas, O. Wiest, P. Helquist, Single-flask multicomponent synthesis of highly substituted $\alpha$-pyrones via a sequential enolate arylation and alkenylation strategy. Org. Lett. 18, 5724-5727 (2016).

${ }^{2}$ B. Pieber, S. T. Martinez, D. Cantillo, C. O. Kappe, In situ generation of diimide from hydrazine and oxygen: continuous-flow transfer hydrogenation of olefins. Angew. Chem. Int. Ed. 52, $10241-10244$ (2013).

${ }^{3}$ S. Baader, P. E. Podsiadly, D. J. Cole-Hamilton, L. J. Goossen. Synthesis of tsetse fly attractants from a cashew nut shell extract by isomerising metathesis. Green Chem. 16, 4885-4890 (2014).

${ }^{4}$ N. Kalutharage, C. S. Yi, Scope and mechanistic analysis for chemoselective hydrogenolysis of carbonyl compounds catalyzed by a cationic ruthenium hydride complex with a tunable phenol ligand. J. Am. Chem. Soc. 137, 11105-11114 (2015).

${ }^{5}$ G. Zhao, L.-Z. Yuan, M. Alami, O. Provot, Chlorotrimethylsilane and sodium iodide: a remarkable metal-free association for the desulfurization of benzylic dithioketals under mild conditions. Adv. Synth. Catal. 360, 2522-2536 (2018).

6 D.-H. Lee, K.-H. Kwon, C. S. Yi, Dehydrative C-H alkylation and alkenylation of phenols with alcohols: expedient synthesis for substituted phenols and benzofurans. $J$. Am. Chem. Soc. 134, 7325-7328 (2012).

${ }^{7}$ G. Li, P. Gao, X. Lv, C. Qu, Q. Yan, Y. Wang, S. Yang, J. Wang, Synthesis of $m$-alkylphenols via a ruthenium-catalyzed $\mathrm{C}-\mathrm{H}$ bond functionalization of phenol derivatives. Org. Lett. 19, 2682-2685 (2017).

${ }^{8}$ N. Hu, K. Li, Z. Wang, W. Tang, Synthesis of chiral 1,4-benzodioxanes and chromans by enantioselective palladium-catalyzed alkene aryloxyarylation reactions. Angew. Chem. Int. Ed. 55, 5044-5048 (2015).

9 J. Luo, B. Hu, A. Sam, T. L. Liu, Metal-free electrocatalytic aerobic hydroxylation of arylboronic acids. Org. Lett. 20, 361-364 (2018).

10 R. K. Dieter, Y. J. Lin, J. W. Dieter, 1,3-Carbonyl transposition methodology employing $\alpha$-oxo ketene dithioacetals: application in the synthesis of phenols and ( \pm )-myodesmone. J. Org. Chem, 49, 3183-3195 (1984).

11 Z. Wang, A. Orellana, Coonvenient access to meta-substituted phenols, by palladium-catalyzed Suzuki-Miyaura cross-coupling and oxidation. Chem. Eur. J. 23, 11445-11449 (2017)

12 T. Akama, Y.-K. Zhang, Y. R. Freund, P. Berry, J. Lee, E. E. Easom, R. T. Jacobs, J. J. Plattner, M. J. Witty, R. Peter, T. G. Rowan, K. Gillingwater, R. Brun, B. Nare, L. Mercer, M. Xu, J. Wang, H. Liang, Identification of a 4-fluorobenzyl L-valinate amide benzoxaborole (AN11736) as a potential development candidate for the treatment of animal African trypanosomiasis (AAT). Bioorg. Med. Chem. Lett. 28, 6-10 (2018).

13 G. Appendino, G. Cravotto, G. B. Giovenzana, G. Palmisano, A straightforward entry into polyketide monoprenylated furanocoumarins and pyranocoumarins. J. Nat. Prod. 62, 1627-1631 (1999)

14 A. Fischer, G. N. Henderson, R. J. Thompson, Ipso nitration. XIX. formation of cyclohexadiene adducts from nitration of 4-ethyltoluene and 1,4-diethylbenzene in nitric acid and acetic anhydride. Aust. J. Chem, 31, 1241-1247 (1978)

15 C.-C. Chan, Y.-W. Chen, C.-S. Su, H.-P. Lin, C.-F. Lee, Green catalysts derived from agricultural and industrial waste products: the preparation of phenols from $\mathrm{CsOH}$ and aryl iodides using $\mathrm{CuO}$ on mesoporous silica. Eur. J. Org. Chem. 36, 7288-7293 (2011)

${ }^{16}$ K. Saito, Y. Onizawa, H. Kusama, N. Iwasawa, Rhenium(I)-catalyzed cyclization 
of silyl enol ethers containing a propargyl carboxylate moiety: versatile access to highly substituted phenols. Chem. Eur. J. 16, 4716-4720 (2010).

17 F. E. Boyer, J. V. N. V. Prasad, J. M. Domagala, E. L. Ellsworth, C. Gajda, S. E. Hagen, L. J. Markoski, B. D. Tait, E. A. Lunney, A. Palovsky, D. Ferguson, N. Graham, T. Holler, D. Hupe, C. Nouhan, P. J. Tummino, A. Urumov, E. Zeikus, G. Zeikus, S. J. Gracheck, J. M. Sanders, S. VanderRoest, J. Brodfuehrer, K. Iyer, M. Sinz, S. V. Gulnik, J. W. Erickson, 5,6-Dihydropyran-2-ones possessing various sulfonyl functionalities: potent nonpeptidic inhibitors of HIV protease. J. Med. Chem. 43, 843-858 (2000). 


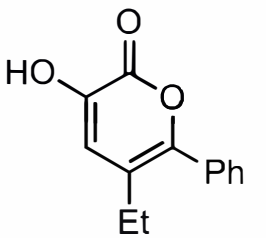

22 - S1

Current Data Parameters
NAME
XZ-2-206

PROCNO

F2 - Acquisition Parameters

Date_ 20180824

Time $11.45 \mathrm{~h}$

INSTRUM

PROBHD Z566801 0015

PULPROG zg30

TD 65536

SOLVENT

$\begin{array}{lr}\text { NS } & \text { CDCI3 } \\ \text { DS } & 16\end{array}$

DS

$10504.202 \mathrm{~Hz}$

FIDRES $\quad 0.320563 \mathrm{~Hz}$

$\mathrm{AQ} \quad 3.1195135 \mathrm{sec}$

RG

DW

$\mathrm{DE}$

TE

TD0

SFO1

NUC1

$\mathrm{P} 1$

P1
PLW1

161

47.600 usec
6.50 usec

$296.0 \mathrm{~K}$

$1.00000000 \mathrm{sec}$

$700.0115500^{1} \mathrm{MHz}$

$1 \mathrm{H}$

12.88 usec

$40.00000000 \mathrm{~W}$

F2 - Processing parameters

SI 131072

SF $\quad 700.0070170 \mathrm{MHz}$

WDW

B

GB

0
0 $\mathrm{~Hz}$

1.00

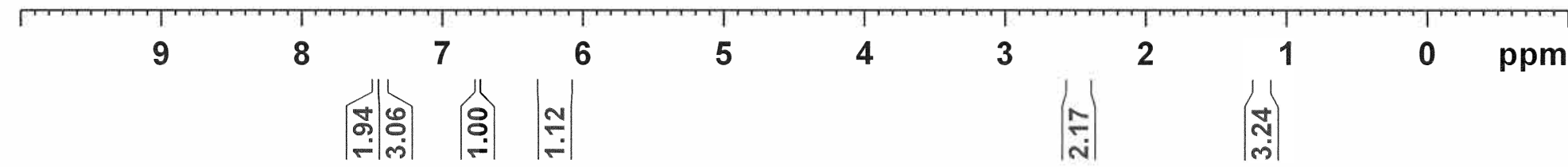




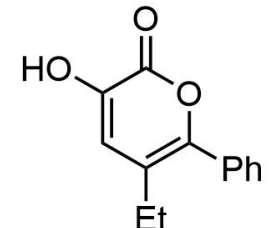

22 - S1
Current Data Parameter

EXPNO

PROCNO

F2 - Acquisition Parameters

20180824

Time $\quad 11.56 \mathrm{~h}$

INSTRUM spect

PROBHD Z566801 0015

PULPROG Zgpg30

$\mathrm{TD}$

65536

SOLVENT

NS

DS

SWH

FIDRES

$\mathrm{AQ}$
$\mathrm{RG}$

DW

DE

TE

D11

TDO

$\mathrm{SFO} 1$

NUC1

P1

PLW1

$\mathrm{SFO} 2$

NUC2

CPDPRG [2

PCPD2

PLW2

PIW12

PLW13

SI 131072

SF $\quad 176.0169002 \mathrm{MHz}$

WDW

SSB

LB

GB

$\mathrm{O} \mathrm{Hz}$ 
F2 - Acquisition Parameters

Date_ 20190321

Time- $10.48 \mathrm{~h}$

INSTRUM spect

PROBHD Z151340_0001

PULPROG Z $\quad$ zpg30

SOLVENT

22 - S2

SFO1

SFO1

NUC1

P1

PLW1

$\mathrm{SFO} 2$

NUC2

CPDPRG [ 2

PCPD2

PLW2

PLW12

$0.03000000 \mathrm{sec}$

$176.0362620 \mathrm{MHz}$

$$
\begin{array}{r}
13 \mathrm{C} \\
11.25
\end{array}
$$

29.00000 .25 use $29.00000000 \mathrm{~W}$

$700.0098000 \mathrm{MHz}$

PLW13$$
\text { waltz } 16
$$

$$
65.00 \text { usec }
$$

$13.00000000 \mathrm{~W}$

$0.27768999 \mathrm{~W}$

$0.13982999 \mathrm{~W}$

F2 - Processing parameters

SI 131072

SF $\quad 176.0169002 \mathrm{MHz}$

WDW EM

$\begin{array}{lll}\text { SSB } & 0 & 1.00 \mathrm{~Hz} \\ \mathrm{LB} & 0 & \end{array}$

0

1.40

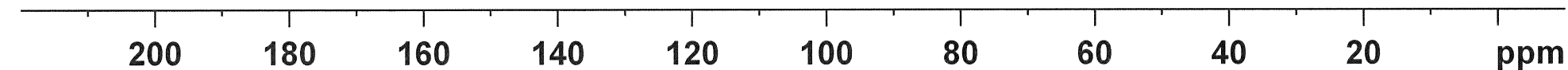



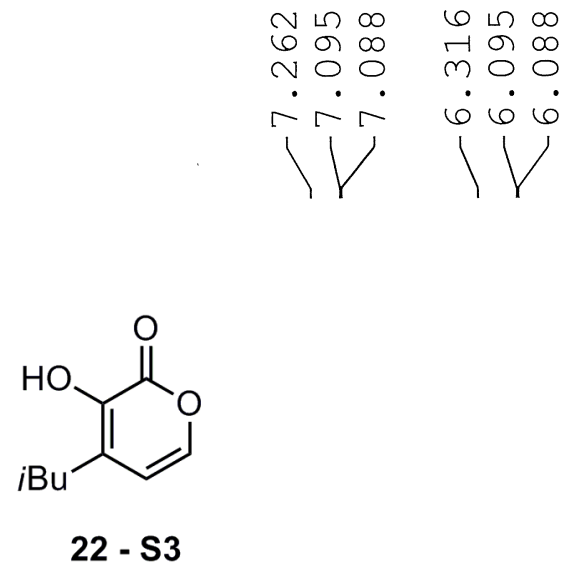

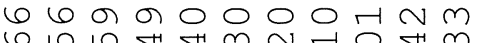

m लन नून न न न

रतनमतन

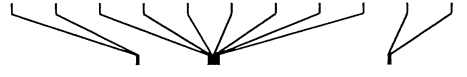

Current Data Parameters NAME

EXPNO $\mathrm{Xz}-3-25$

PROCNO

F2 - Acquisition Parameters

Date_ 20181206

Time 13.20

INSTRUM spect

PROBHD Z151340_0001

PULPROG $\quad$ zg30

SOLVENT

NS

16

DS

SWH $\quad 10504.202 \mathrm{~Hz}$

FIDRES $\quad 0.320563 \mathrm{~Hz}$

$\mathrm{AQ} \quad 3.1195135 \mathrm{sec}$

5.

47.600 usec $\begin{array}{ll}\mathrm{DE} & 10.00 \mathrm{usec} \\ \mathrm{TE} & 298.2 \mathrm{~K}\end{array}$

D1 $1.00000000 \mathrm{sec}$

TD0

$\mathrm{SFO1}$

SFO1

1

$700.0115500 \mathrm{MHz}$

$1 \mathrm{H}$

PLW 1

$13.00000000 \mathrm{~W}$

F2 - Processing parameters

SI 131072

SF $\quad 700.0070147 \mathrm{MHz}$

WDW

$\begin{array}{lll}\text { SSB } & 0 \\ \text { LB } & 0 & \mathrm{~Hz}\end{array}$

$\begin{array}{lll}\text { GB } & 0 \\ \text { PC } & \end{array}$

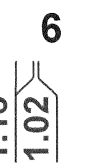



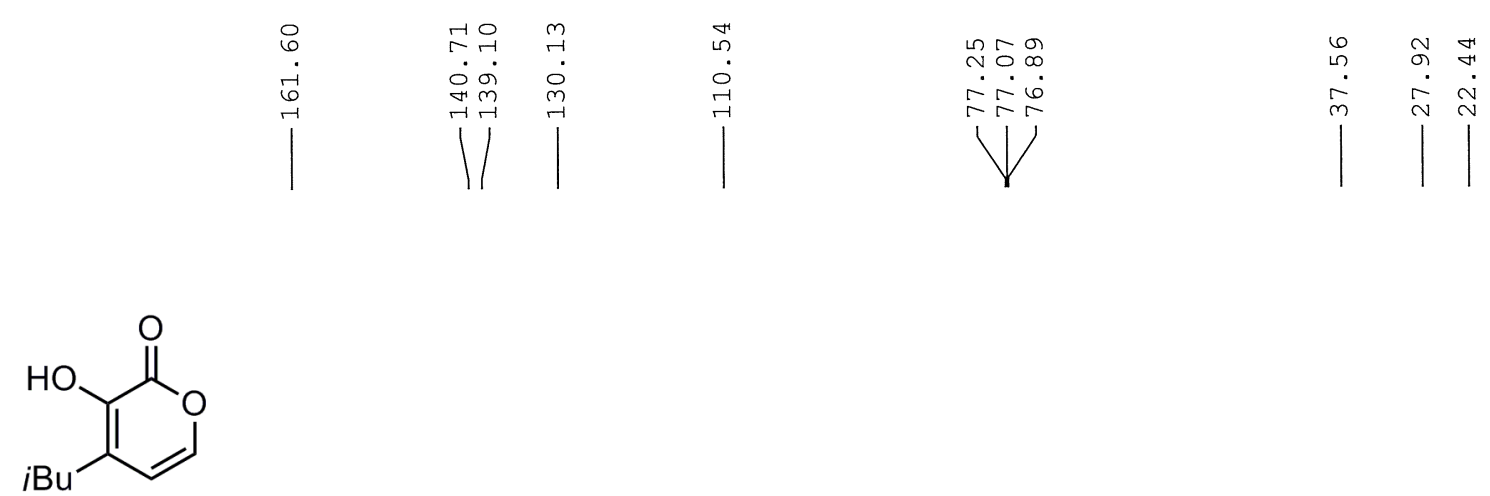

$$
\begin{array}{lr}
\text { NAME } & \mathrm{XZ}-3-25 \\
\text { EXPNO } & 2 \\
\text { PROCNO } & 1
\end{array}
$$

Current Data Parameters

F2 - Acquisition Parameters

Date_ 20181206

Time ${ }^{-} \quad 13.26$ h

INSTRUM spect

PROBHD Z151340_0001

zgpg 30
65536

TD 65536

22 - S3 


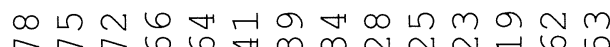

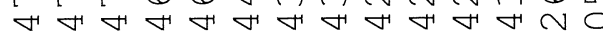

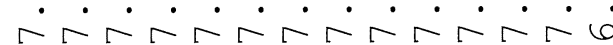

$\longrightarrow$<smiles>CCc1c(-c2ccccc2)oc(=O)c(O)c1CC(C)C</smiles>

22 - S4

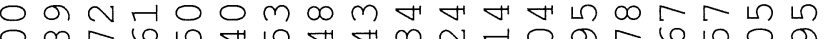

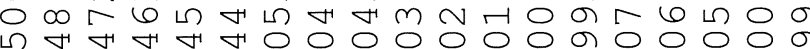
$\dot{\sim} \dot{\sim} \dot{N} \dot{N} \dot{N} \dot{N} \dot{N} \dot{N} \dot{\sim} \dot{H} \dot{H} \dot{H} \dot{H}$

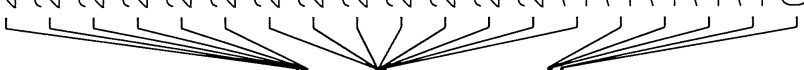

Current Data Parameters NAME

EXPNO $\mathrm{xz}-3-171-1$ PROCNO

F2 - Acquisition Parameters

Date_ 20190516

Time 17.51 h

INSTRUM spect

PROBHD Z151340_0001

PULPROG Zg30

TD 65536

SOLVENT CDCl3

NS

$\begin{array}{lr}\text { DS } & 2 \\ \text { SWH } & 10504.202 \mathrm{~Hz}\end{array}$

FIDRES $\quad 0.320563 \mathrm{~Hz}$

$\mathrm{AQ} \quad 3.1195135 \mathrm{sec}$

$\begin{array}{lr}\text { DW } & 47.600 \text { usec } \\ \text { DE } & 10.00 \text { usec }\end{array}$

$\mathrm{TE}$

D1

TDO

$\mathrm{SFO1}$

PLW1

$303.2 \mathrm{~K}$

$1.00000000 \mathrm{sec}$

$700.0115500 \mathrm{MHz}$

$1 \mathrm{H}$
9.50 usec

$13.00000000 \mathrm{~W}$

2 - Processing parameter

SI 131072

SF $\quad 700.0070160 \mathrm{MHz}$

WDW

EM

$0.30 \mathrm{~Hz}$

GB

PC

1.00
9

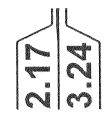

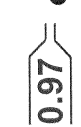

!

5

\begin{abstract}
4
4
\end{abstract}

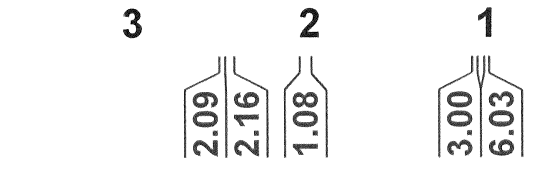




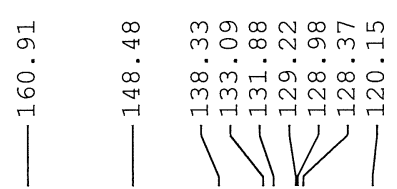

PE

22 - S4
임ำ

$\dot{m} \stackrel{\infty}{N} \underset{N}{\sim} \stackrel{m}{\sim}$

$1|1|$
Current Data Parameters

NAME

EXPNO

PROCNO $\mathrm{Xz}-3-171-1$

1

- Acquisition Parameters

Date_ 20190516

Time $\quad 17.55 \mathrm{~h}$

INSTRUM spect

PROBHD Z151340 0001

PULPROG Zgpg30

TD 65536

SOLVENT CDCl3

DS

SWH

FIDRES

$A Q$

RG

DW

$\mathrm{DE}$

TE
D1
D11

D11

SFO1

NUC1

$\mathrm{P} 1$
$\mathrm{PLW} 1$

PLW1
SFO2

$\mathrm{SFO} 2$
$\mathrm{NUC} 2$

CPDPRG [2

PCPD2

PLW2

PIW12

PLW13

F2 - Processing parameters

SI 131072

$\begin{array}{lc}\text { SF } & 176.0169002 \\ \text { WDW } & \text { EM }\end{array}$

SSB

$L B$

GB
$42613.637 \mathrm{~Hz}$

$1.300465 \mathrm{~Hz}$

$0.7689557 \mathrm{sec}$

11.733 usec

18.00 use $303.2 \mathrm{~K}$

$2.00000000 \mathrm{sec}$ $0.03000000 \mathrm{sec}$

$176.0362620 \mathrm{MHz}$ $13 \mathrm{C}$

29.00000000 use $700.0098000 \mathrm{~W}$$$
\begin{array}{r}
1 \mathrm{H} \\
\text { waltz } 16
\end{array}
$$

65.00 usec

$13.00000000 \mathrm{~W}$

$0.27768999 \mathrm{~W}$

$1.00 \mathrm{~Hz}$

1.40

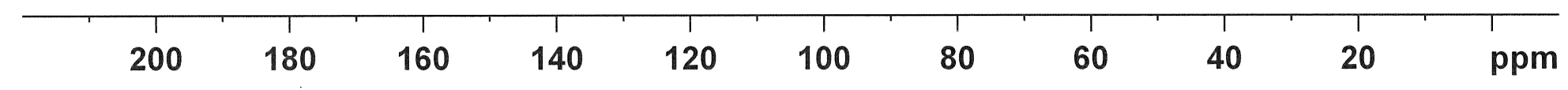


No v

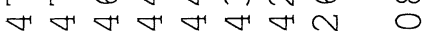

$\sin r a r a r$

$\rightarrow$

22 - S5

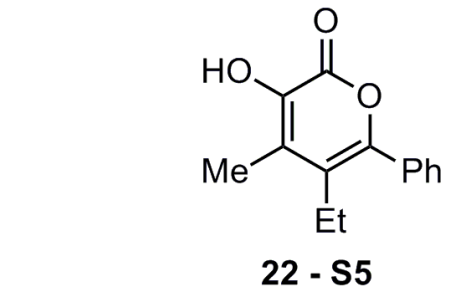

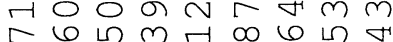

a

$\dot{\sim} \dot{\sim} \dot{\sim} \dot{\vec{H}} \dot{\vec{H}}$

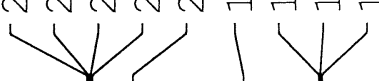




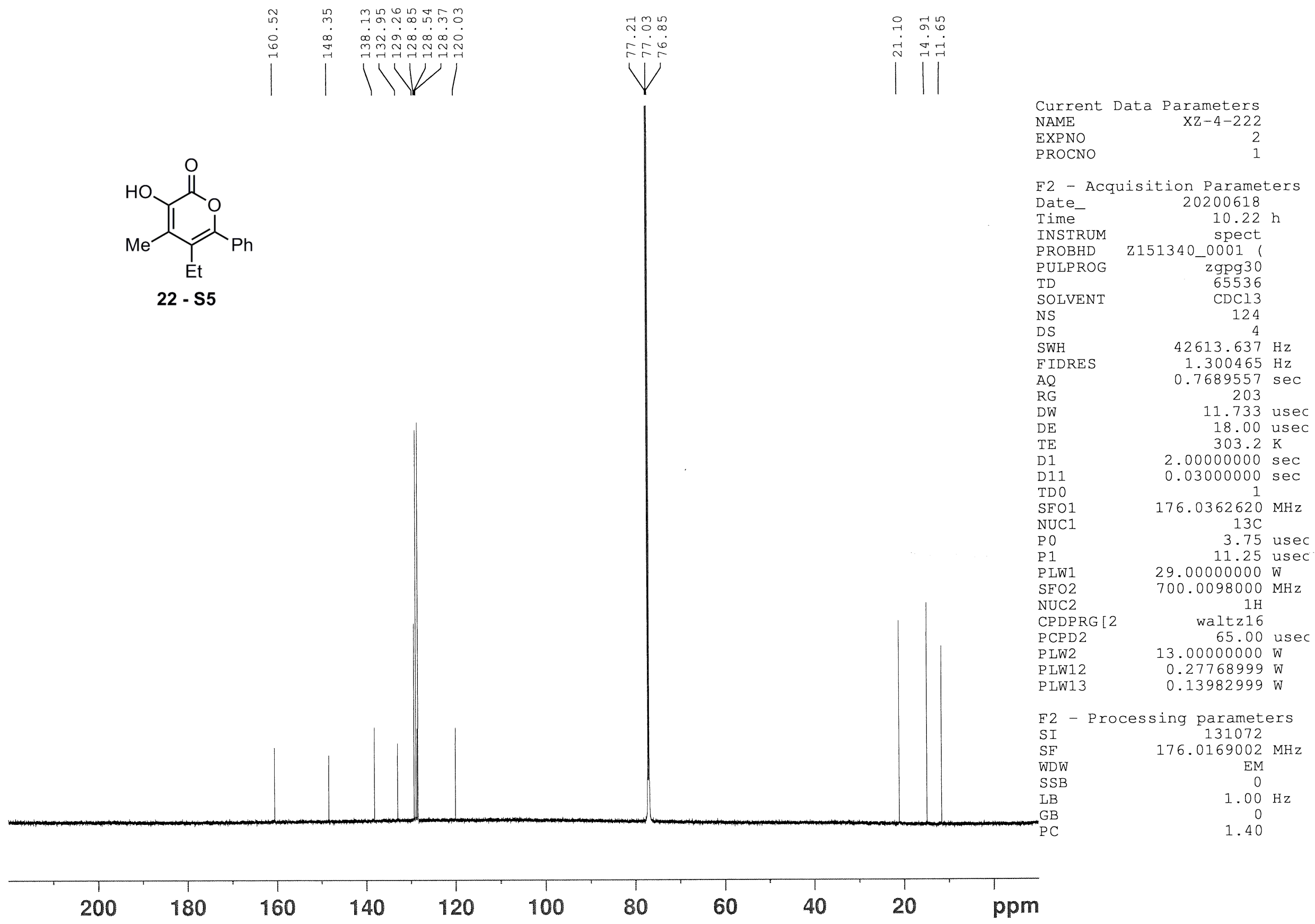




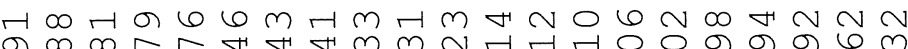

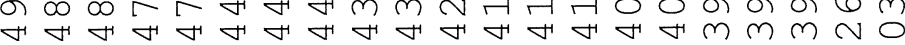

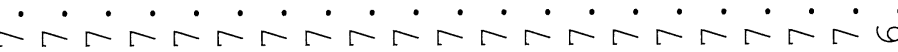

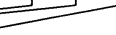

다유 ॠ न न

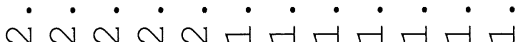

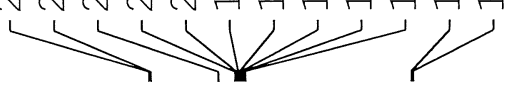

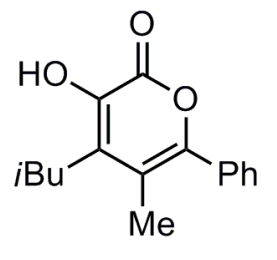

F2 - Acquisition Parameters

Date_ 20190714

Time $16.54 \mathrm{~h}$

INSTRUM spect

PROBHD Z151340_0001

PULPROG Zg30

TD

$\mathrm{CDCl} 3$

SOLVENT

NS

CDCl 16

DS

FIDRES

$10504.202 \mathrm{~Hz}$

$10504.202 \mathrm{~Hz}$
$0.320563 \mathrm{~Hz}$

$3.1195135 \mathrm{sec}$

47.600 usec

20.00 use $303.4 \mathrm{~K}$

$1.00000000 \mathrm{sec}$

$700.0115500^{1} \mathrm{MHz}$ $15500 \mathrm{MHz}$
$1 \mathrm{H}$

$13.00000000 \mathrm{~W}$

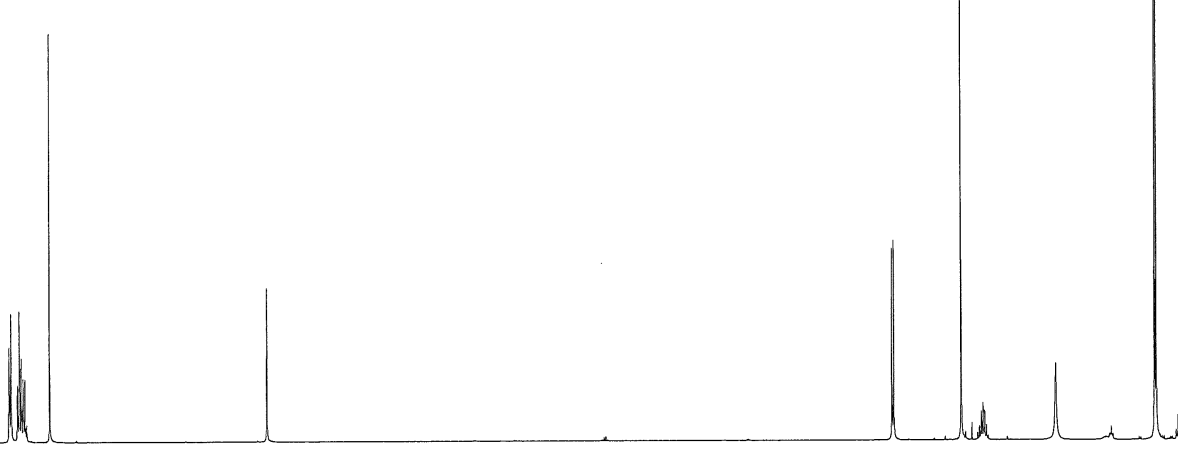

- Processing parameter

SI 131072

SF $\quad 700.0070167 \mathrm{MHz}$

WDW

SSB

0

$$
\text { no }
$$

${ }_{0}^{0} \mathrm{~Hz}$

LB $\quad 0$

$\mathrm{PC}$

1.00

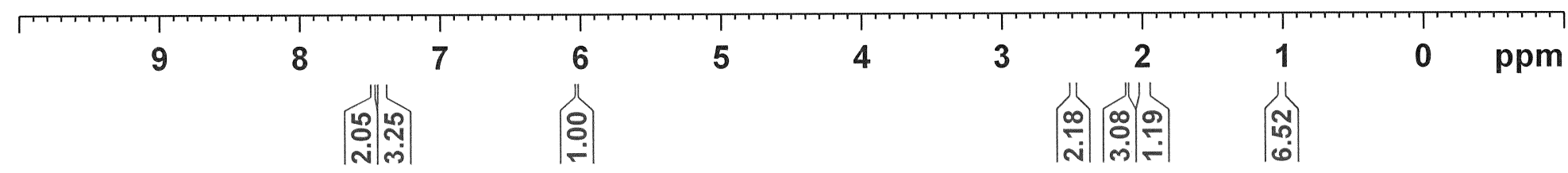



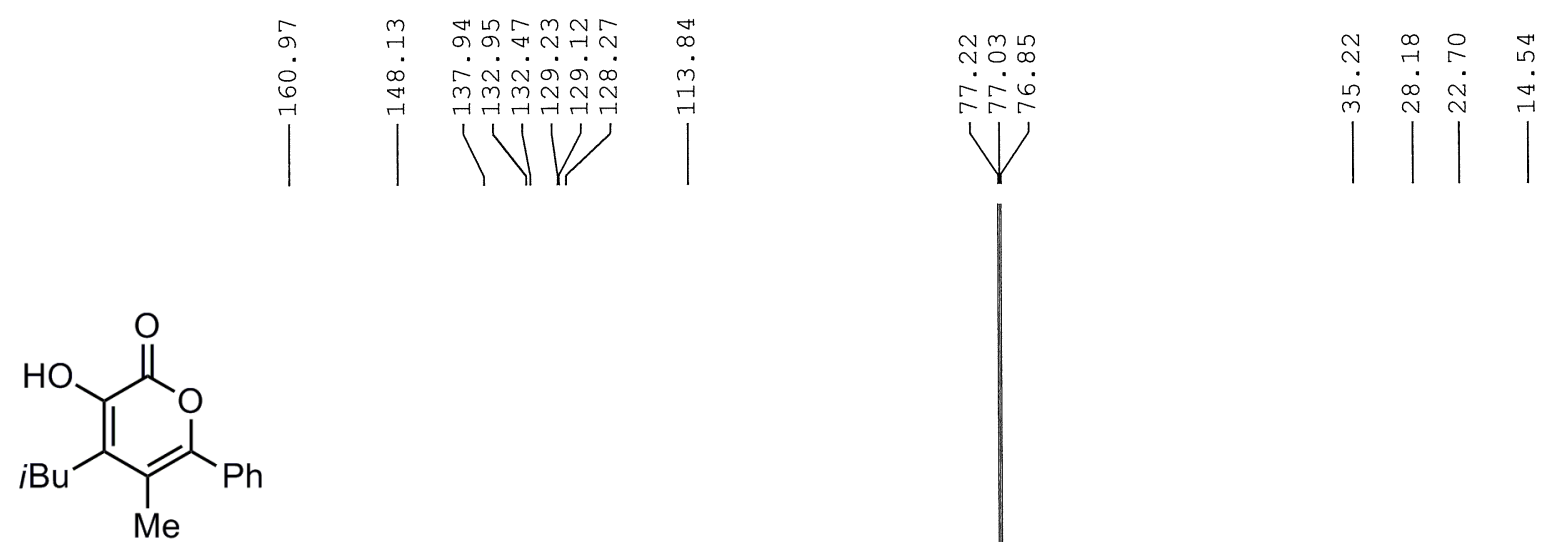

Current Data Parameters
NAME
Xz-3-242

EXPNO

F2 - Acquisition Parameters

Date_ 20190714

Time $\quad 17.01 \mathrm{~h}$

INSTRUM spect

PROBHD Z151340_0001

PULPROG Zgpg30

TD 65536

SOLVENT CDC13

22 - S6 


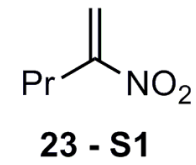

23 - S1

$\begin{array}{lr}\text { F2 - Acquisition } & \text { Parameters } \\ \text { Date_ } & 20191130 \\ \text { Time } & 12.41 \mathrm{~h} \\ \text { INSTRUM } & \text { spect } \\ \text { PROBHD } & \text { r151340_0001 l } \\ \text { PULPROG } & \text { zg30 } \\ \text { TD } & 65536 \\ \text { SOLVENT } & \text { CDC13 } \\ \text { NS } & 16 \\ \text { DS } & 2 \\ \text { SWH } & 10504.202 \mathrm{~Hz} \\ \text { FIDRES } & 0.320563 \mathrm{~Hz} \\ \text { AQ } & 3.1195135 \mathrm{sec} \\ \text { RG } & 18 \\ \text { DW } & 47.600 \mathrm{usec} \\ \text { DE } & 20.00 \mathrm{usec} \\ \text { TE } & 303.2 \mathrm{~K} \\ \text { D1 } & 1.00000000 \mathrm{sec} \\ \text { TD0 } & 1 \\ \text { SFO1 } & 700.0115500 \mathrm{MHz} \\ \text { NUC1 } & 1 \mathrm{H} \\ \text { P0 } & 3.17 \mathrm{usec} \\ \text { P1 } & 9.50 \mathrm{usec} \\ \text { PIN1 } & 13.0000000 \mathrm{H}\end{array}$

PLW 1 $13.00000000 \mathrm{~W}$

- Processing parameter SI 131072 SF $\quad 700.0070163 \mathrm{MHz}$

LB

GB 


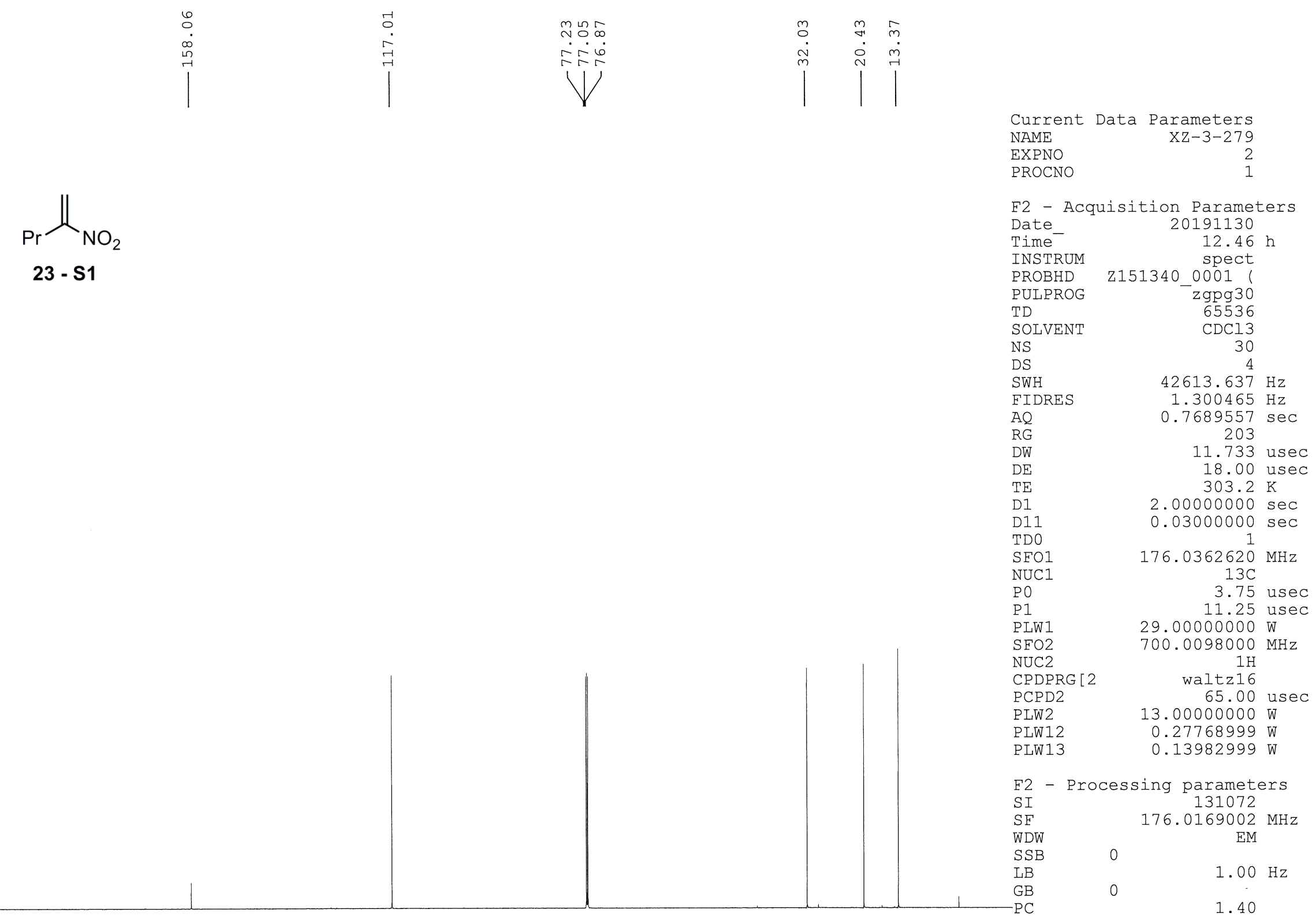

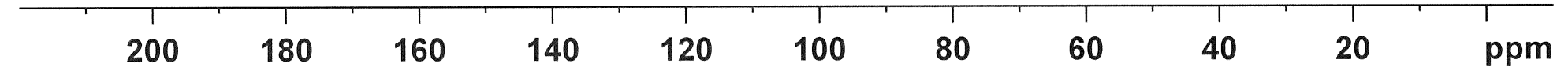


$\infty \infty \infty$ a $\sim m$ $m m \sim \sim \sim \sigma$ $\cdot \cdot$ - $\dot{0} \dot{0} \dot{0} \dot{0}$

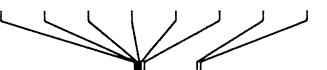

$$
\mathrm{Et} \sim \mathrm{NO}_{2}
$$

23 - $\mathrm{S2}$

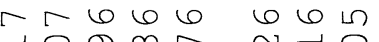

m $m \sim N \sim \mathrm{N} N$

$\cdot \cdot \cdot \cdot \dot{\sim} \cdot$

$N \sim N N \sim$

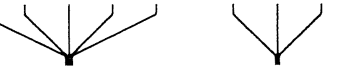

Current Data Parameters

NAME

EXPNO

PROCNO

F2 - Acquisition Parameters Date_ 20200620

Time

INSTRUM

INTSUM

ROBHD

TD

NS

DS

SWH

FIDRES

AQ

RG

DW

TE

D 1

NFO1

$\mathrm{PO}$

$\mathrm{P} 1$

PLW1

F2 - Processing parameters

SI 131072

SF $\quad 700.0070158 \mathrm{MHz}$

WDW

SSB

$\mathrm{LB}$
$\mathrm{GB}$

PC $\mathrm{XZ}-4-249$ 1
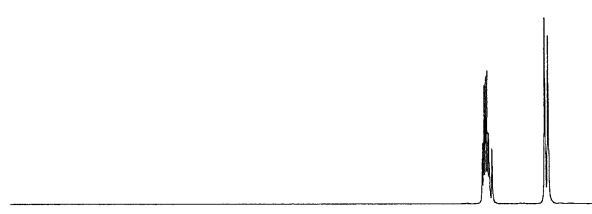

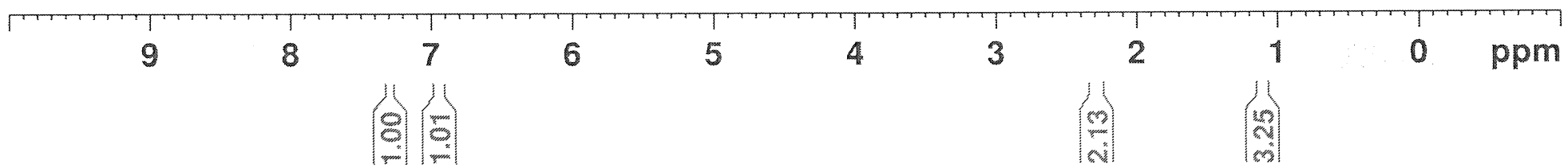



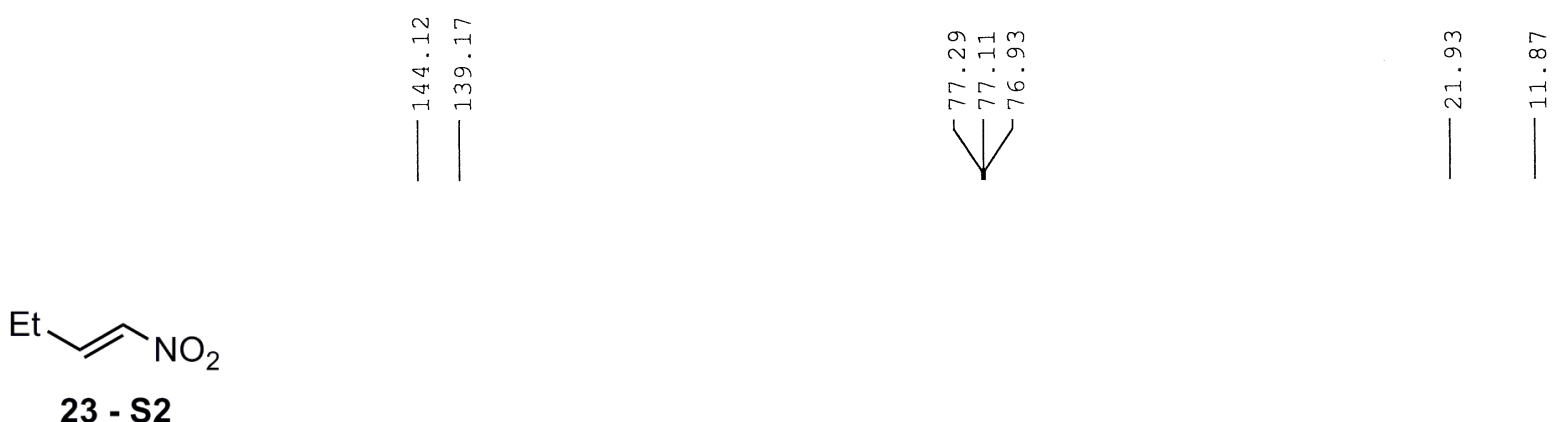

Current Data Parameter

NAME

EXPNO

PROCNO

$\mathrm{XZ}-4-249$

F2 - Acquisition Parameters

Date_ 20200620

Time $\quad 14.20 \mathrm{~h}$

INSTRUM spect

PROBHD Z151340_0001 (

PULPROG zgpg30

$\begin{array}{ll}\text { TD } & 65536 \\ \text { SOLVENT } & \text { CDCl3 }\end{array}$

SOLVENT

$\mathrm{CDC} 13$

DS

SWH

FIDRES

AQ

DW

DE

$\mathrm{TE}$

D11

TD 0

$\mathrm{SFO} 1$
$\mathrm{NUC1}$

$\mathrm{P} O$

P1

PLW1

$\mathrm{SFO} 2$

NUC2

CPDPRG [ 2

PCPD 2

PLW2

PLW12

PLW13

$42613.637 \mathrm{~Hz}$

$1.300465 \mathrm{~Hz}$

$0.7689557 \mathrm{sec}$

203

11.733 usec

18.00 usec $303.2 \mathrm{~K}$

$2.00000000 \mathrm{sec}$

$0.03000000 \mathrm{sec}$

$0.03000000 \mathrm{sec}$

$176.0362620 \mathrm{MHz}$ $13 \mathrm{C}$ 3.75 usec
11.25 usec $29.00000000 \mathrm{~W}$ $700.0098000 \mathrm{MHz}$ O. O. $277682999 \mathrm{~W}$ 2 - Processing parameter SI $\quad 131072$ SF $\quad 176.0169002 \mathrm{MHz}$ WDW SSB

LB GB

EM
0
$1.00 \mathrm{~Hz}$
0
1.40


ข

ㄴ.

RAR $-\dot{0} \dot{0} \dot{0} 6$

31

${ }^{\mathrm{Bu}} \mathrm{NO}_{2}$

23 - S3
のたレみ

मनंमें

. $\cdot$.

각닥

Current Data Parameters

F2 - Acquisition Parameters

Date_ 20191130

$\begin{array}{ll}\text { Time } & 13.17 \mathrm{~h}\end{array}$

INSTRUM spect

PULPROG Zg30

$\mathrm{zg} 30$
65536

SOLVENT

NS

DS

FIDRES

$\mathrm{CDCl} 3$

TIDES

$\mathrm{AQ}$

RG

DW

$\mathrm{DE}$

TE

TD0

SFO1

NUC1

NUC

P0

PLW1

16
2
$10504.202 \mathrm{~Hz}$

$0.320563 \mathrm{~Hz}$

$3.1195135 \mathrm{sec}$

47.600 usec 20.00 usec $303.2 \mathrm{~K}$

$1.00000000 \mathrm{sec}$

$700.0115500^{1} \mathrm{MHz}$

$1 \mathrm{H}$
3.17 usec

3.17 usec
9.50 usec

F2 - Processing parameter

SI 131072

SF $\quad 700.0070158 \mathrm{MHz}$

WDW

SSB

0

LB $\quad 0 \mathrm{~Hz}$

GB

$\mathrm{Hz}$

no

$\mathrm{PC}$

1.00

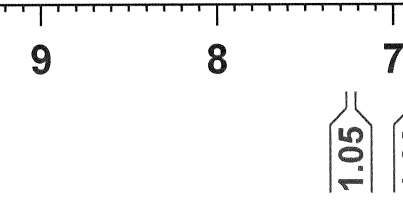

1)

6

5

4

3

2

lat

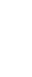




\section{${ }^{t \mathrm{Bu}} \sim \mathrm{NO}_{2}$}

23 - 53
Current Data Parameters $\mathrm{XZ}-2-244$

EXPNO

F2 - Acquisition Parameters

Date_ 20191130

Time 13.20

INSTRUM spect

PROBHD Z151340 0001

PULPROG Z zgpg30

65536

SD

NS

DS

SWH

AQ

RG

DW

$\mathrm{DE}$

D1

D11

TDO

$\mathrm{SFO1}$

NUC1

$\mathrm{PO}$

PLW1

PLW1
SFO2

NUC2

CPDPRG [ 2

PCPD2

PLW2

PLW12

PLW13

$42613.637 \mathrm{~Hz}$

$1.300465 \mathrm{~Hz}$

$0.7689557 \mathrm{sec}$ 203

11.733 usec 18.00 usec $303.2 \mathrm{~K}$

$2.00000000 \mathrm{sec}$ $0.03000000 \mathrm{sec}$ 1 $176.0362620 \mathrm{MHz}$ 3.75 usec 11.25 usec 29.00000000 $700.0098000 \mathrm{MHz}$

$$
\begin{array}{r}
1 \mathrm{H} \\
\text { waltz } 16 \\
65.00
\end{array}
$$

3.00000000 usec

$0.27768999 \mathrm{~W}$

$0.13982999 \mathrm{~W}$

F2 - Processing parameters

SI 131072

SF $\quad 176.0169002 \mathrm{MHz}$

WDW

SSB

LB

$G B$
$-P C$

$1.00 \mathrm{~Hz}$

1.40 

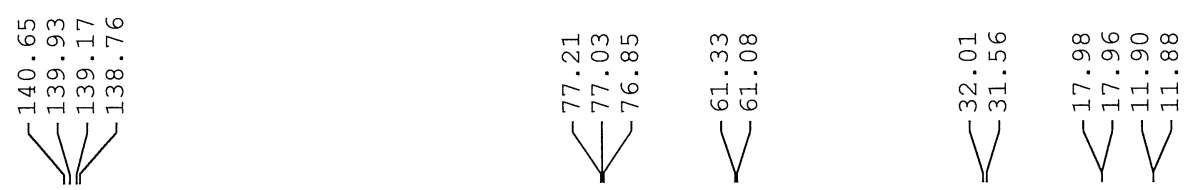

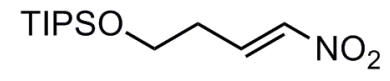

23 - 54
Current Data Parameters

$$
\text { NAME }
$$

EXPNO

PROCNO

F2 - Acquisition Parameters

Date_ 20191004

Time- 12.12

INSTRUM

PROBHD

PULPROG

TD

SOLVENT

NS

DS

FIDRES

$\mathrm{AQ}$

$\mathrm{RG}$

DW

$\mathrm{DE}$

$\mathrm{TE}$

D1

T110

$\mathrm{SFO1}$

NUC1

$\mathrm{PO}$

P1

PLW1

SFO2
NUC2

CPDPRG [2

PCPD2

PLW2

PLW12

2151340_0001

zgpg 30
65536

$\mathrm{CDCl} 3$
59 59

$42613.637 \mathrm{~Hz}$

$1.300465 \mathrm{~Hz}$

$0.7689557 \mathrm{sec}$ 203
11.733

11.733 usec 18.00 usec $303.2 \mathrm{~K}$

$2.00000000 \mathrm{sec}$ $0.03000000 \mathrm{sec}$

$176.0362620^{1} \mathrm{MHz}$

$$
13 \mathrm{C}
$$

3.75 usec

3.75 usec
11.25 usec

$29.00000000 \mathrm{~W}$

$700.0098000 \mathrm{MHz}$

$1 \mathrm{H}$

waltz16

65.00 usec

$13.00000000 \mathrm{~W}$

$0.27768999 \mathrm{~W}$

$0.13982999 W$

F2 - Processing parameters

SI 131072

$\begin{array}{ll}\mathrm{S} F & 176.0169002 \mathrm{MHz}\end{array}$

WDW

SSB $\quad 0$

$1.00 \mathrm{~Hz}$

1.40 


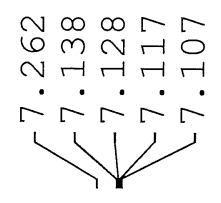

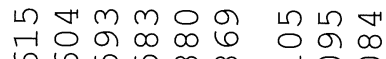

$\dot{\sim} \dot{\sim} \dot{\sim} \dot{-i}$

W

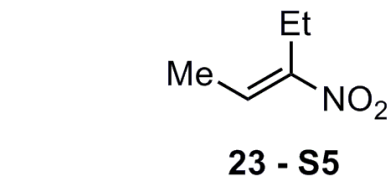

23 - 55

Current Data Parameters

1

F2 - Acquisition Parameters

Date 20191130

Time 12.18

INSTRUM

PROBHD Z151340 0001

PULPROG

TD

zg30
65536

SOLVENT

DS

SWH $\quad 10504.202 \mathrm{~Hz}$

FIDRES $\quad 0.320563 \mathrm{~Hz}$

$\mathrm{AQ} \quad 3.1195135 \mathrm{sec}$

RG

DW

$\mathrm{DE}$

$T E$

TDO

SFO

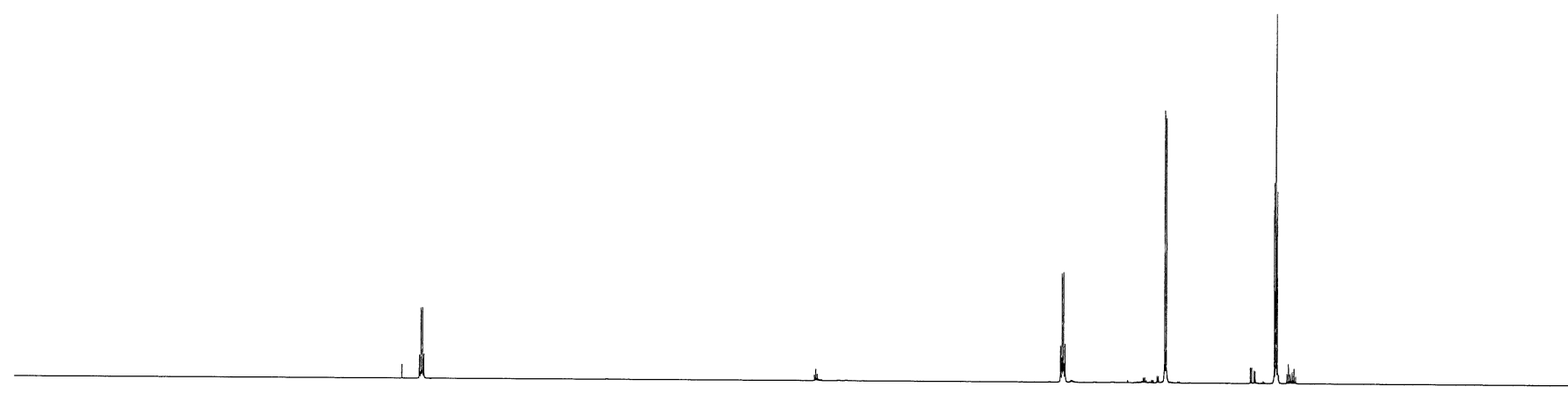

NUC1

P0
P1

PLW1

47.600 usec

20.00 usec

$1.00000000 \mathrm{sec}$

$700.0115500 \mathrm{MHz}$

$1 \mathrm{H}$

3.17 usec

$\begin{aligned} & 9.50 \mathrm{usec} \\ & 13.00000000 \mathrm{~W}\end{aligned}$

F2 - Processing parameters

SI 131072

$\begin{array}{ll}\text { SF } & 700.0070160 \mathrm{MHz}\end{array}$

SSB

GB

no

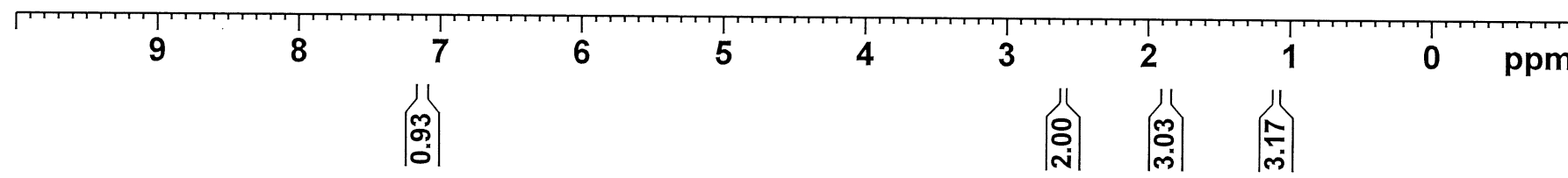




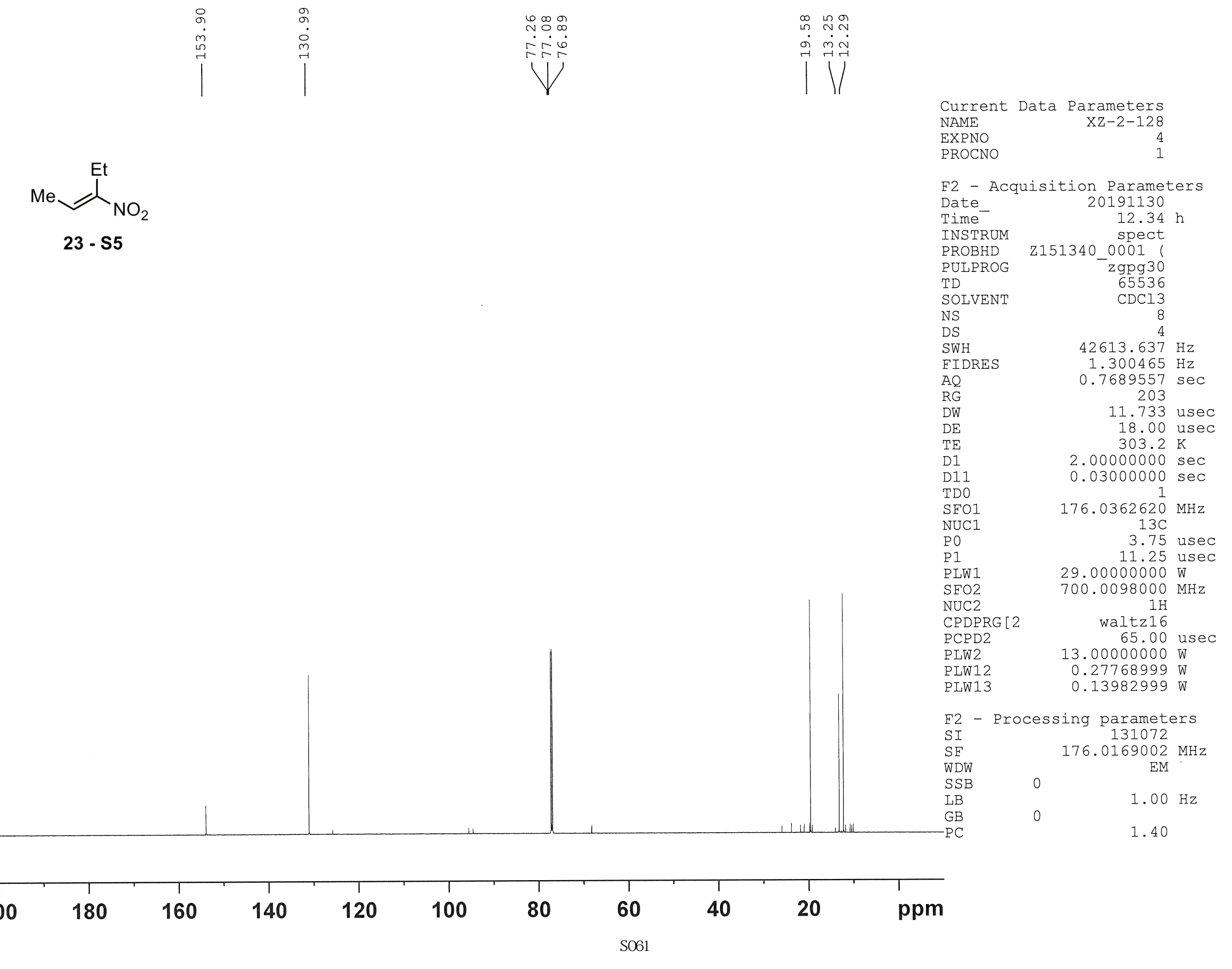


지유

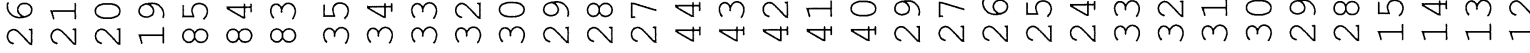

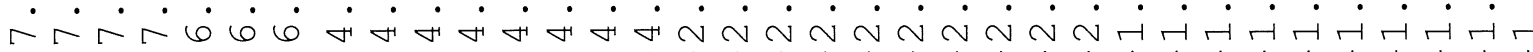
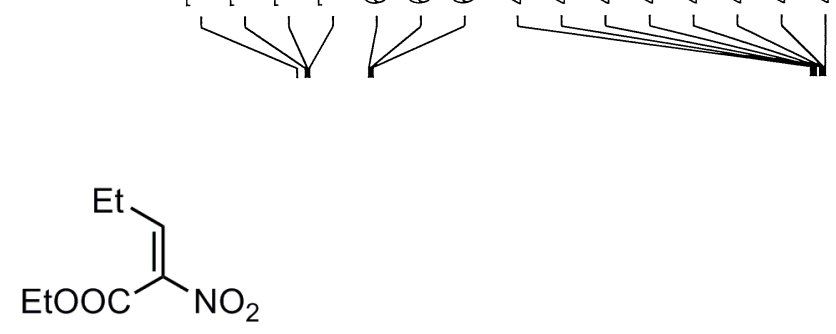

$23-\mathrm{S} 6$

Current Data Parameters NAME EXPNO $\mathrm{XZ}-3-157$ F2 - Acquisition Parameters Date_ 20191130 Time $13.26 \mathrm{~h}$ INSTRUM spect PROBHD Z151340_0001) PULPROG $\quad$ zg30 TD 65536 SOLVENT CDC13

(1)

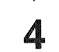

3 疋会会

2 1 

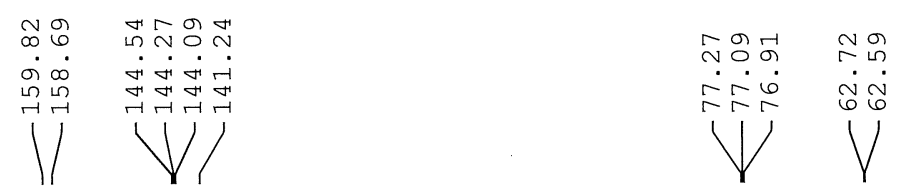

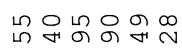

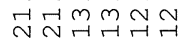

VV

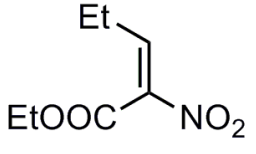

23 - 56

$\begin{array}{lr}\text { NAME } & \mathrm{XZ}-3-157 \\ \text { EXPNO } & 5 \\ \text { PROCNO } & 1\end{array}$

F2 - Acquisition Parameters

Date_ 20191130

Time- $13.54 \mathrm{~h}$

INSTRUM spect

PROBHD Z151340_0001

PULPROG zgpg30

TD 65536

SOLVENT CDCl3

NS

SWH $\quad 42613.637 \mathrm{~Hz}$

FIDRES $\quad 1.300465 \mathrm{~Hz}$

AQ $\quad 0.7689557 \mathrm{sec}$

RG

DW

$\mathrm{DE}$

DI

D11

TDO

$\mathrm{SFO1}$

P0

P1
PLW1

$\mathrm{SFO} 2$

NUC2

CPDPRG [2

PCPD2

PLW2

PLW 12

203
11.733 usec

11.733 usec 18.00 use

$2.00000000 \mathrm{sec}$ $0.03000000 \mathrm{sec}$

$176.0362620 \mathrm{MHz}$

$$
13 \mathrm{C}
$$

3.75 usec

11.25 usec

29.00000000

$700.0098000 \mathrm{MHz}$

$$
\begin{array}{r}
1 \mathrm{H} \\
\text { waltz } 16
\end{array}
$$

65.00 usec

$13.00000000 \mathrm{~W}$

$0.27768999 \mathrm{~W}$

$0.13982999 \mathrm{~W}$

F2 - Processing parameters

$\begin{array}{lc}\text { SI } & 131072 \\ \text { SF } & 176.0169002 \mathrm{MHz}\end{array}$

WDW

SSB

176.0169002 MHz

$1.00 \mathrm{~Hz}$

1.40

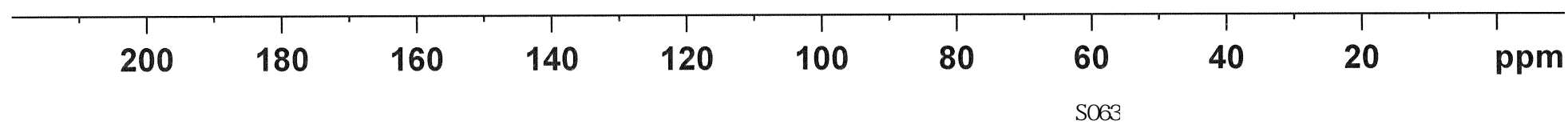




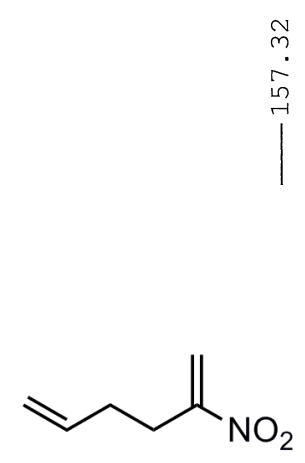

23 - S7
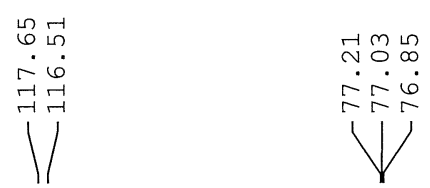

मே

$\left.\right|_{1} ^{m}$
Current Data Parameters

NAME

EXPNO

PROCNO

F2 - Acquisition Parameter

Date

Time-

INSTRUM

PROBHD

PULPROG

TD

SOLVENT

NS

DS

$\mathrm{SWH}$

FIDRES

AQ

RG

DW

TE

D1

TD0

SFO1

NUC1

$\mathrm{PO}$

P1

PLW1

$\mathrm{SFO} 2$

NUC2

CPDPRG [ 2

PCPD2

PLW2

PLW 12

PLW13

F2 - Processing parameters

$\mathrm{SI}$

SF

SSB

SSB
LB
GB

GB
20191016

$17.34 \mathrm{~h}$

spect

2151340_0001 (

zgpg 30

$\mathrm{CDCl} 3$

76

$42613.637 \mathrm{~Hz}$

$1.300465 \mathrm{~Hz}$

$0.7689557 \mathrm{sec}$

11.733 usec 18.00 usec $303.2 \mathrm{~K}$

$2.00000000 \mathrm{sec}$ $0.03000000 \mathrm{sec}$

$176.0362620 \mathrm{MHz}$

$$
\begin{array}{r}
13 \mathrm{C} \\
3.75 \text { usec }
\end{array}
$$
11.25 usec

$29.00000000 \mathrm{~W}$ $700.0098000 \mathrm{MHz}$

$$
\begin{array}{r}
1 \mathrm{H} \\
\text { waltz } 16
\end{array}
$$$$
65.00 \text { usec }
$$

$13.00000000 \mathrm{~W}$

$0.27768999 \mathrm{~W}$

$0.13982999 \mathrm{~W}$$$
\begin{array}{r}
131072 \\
176.0169002
\end{array}
$$

$176.0169002 \mathrm{MHz}$

0

$0 \mathrm{~Hz}$

$\begin{array}{lllllllllll}200 & 180 & 160 & 140 & 120 & 100 & 80 & 60 & 40 & 20 & \text { ppm }\end{array}$




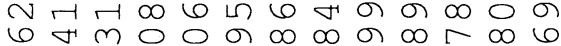

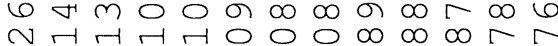

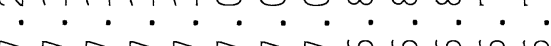

31

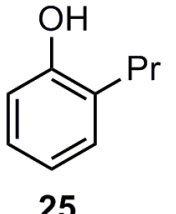

25
$6 \curvearrowleft \forall 6 \curvearrowleft+m m \sim 6 \omega$ 4. क व 0 \%

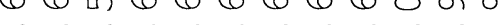

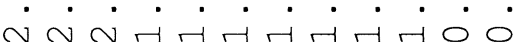

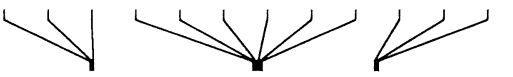

Current Data Parameters NAME

EXPNO

PROCNO

$\mathrm{Xz}-3-280$

- Acquisition Parameters

Date_ 20200618

Time $13.21 \mathrm{~h}$

P151340 spect

PULPROG $\quad \mathrm{zg} 30$

$\begin{array}{lr}\text { PULPROG } & \mathrm{zg} 30 \\ \text { TD } & 65536\end{array}$

SOLVENT CDCl3

NS

SWH

FIDRES

AQ

$\mathrm{DW}$

DE

TE

D 1

TDO

SFO1

NUC1
P 0
P1

PLW1

16
2
$10504.202 \mathrm{~Hz}$
$0.320563 \mathrm{~Hz}$ $0.320563 \mathrm{~Hz}$ $3.1195135 \mathrm{~s}$
16

47.600 usec 20.00 use $303.2 \mathrm{~K}$

$1.00000000 \mathrm{sec}$

$700.0115500 \mathrm{MHz}$

$$
1 \mathrm{H}
$$

$1 \mathrm{H}$
3.17 usec .50 usec

F2 - Processing parameters SI 131072

SF $\quad 700.0070168 \mathrm{MHz}$

WDW EM

SSB

LB

GB

EM
0
$0.30 \mathrm{~Hz}$
0
1.00

$\Gamma$

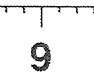

8

7

6

5

60

(c)

43

3

2

(m)

1

ppm 
하 ๘ NHAHRTR $\therefore$ -

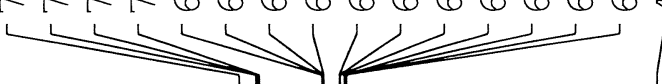
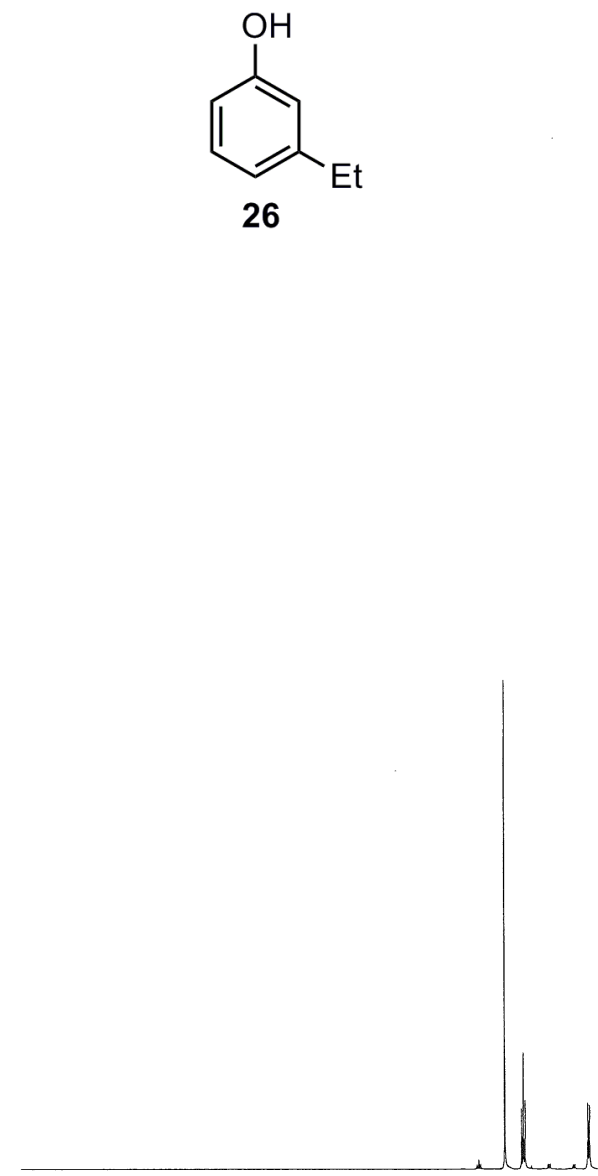

$\infty \infty \sim 6$

$\begin{array}{llll}0 & 6 & 0 & 0 \\ 6 & 6 & 0\end{array}$

$\sim \sim \dot{\sim} \sim$

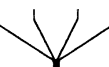

$\circ \infty$

$\sim \sim N$

- $\cdot$

13

Current Data Parameters

NAME

EXPNO

PROCNO

$\mathrm{xz}-3-20$

F2 - Acquisition Parameters

Date__ 20200618

Time $13.05 \mathrm{~h}$

INSTRUM spect

PROBHD Z151340 0001

zg30

TD

SOLVENT

NS

DS

SWH

FIDRES

AQ

RG

DW

DE

IE

TDO
SFO1

NUC1

$\mathrm{PO}$

PI
PLW1

65536
$\mathrm{CDC} 13$

2
$10504.202 \mathrm{~Hz}$

$0.320563 \mathrm{~Hz}$

$0.1195135 \mathrm{sec}$
25.4

47.600 usec

20.00 usec

$303.2 \mathrm{~K}$

$1.00000000 \mathrm{sec}$

1.00000000

$700.0115500 \mathrm{MHz}$

$1 \mathrm{H}$
.17 use

3.17 usec
9.50 usec

$13.00000000 \mathrm{~W}$

F2 - Processing parameters

SI 131072

SF $\quad 700.0070167 \mathrm{MHz}$

WDW

SSB

$\mathrm{LB}$

$\mathrm{GB}$

EM
0
$0.30 \mathrm{~Hz}$
0
1.00

\begin{tabular}{|c|c|c|c|c|c|c|c|c|c|c|}
\hline$\Gamma+1$ & 11 & 11 & $T$ & $T$ & 11 & 11 & 11 & 11 & $T$ & \\
\hline 9 & 8 & 7 & 6 & 5 & 4 & 3 & 2 & 1 & 0 & ppm \\
\hline
\end{tabular}


<smiles>CCc1ccccc1O</smiles>

$\begin{array}{lr}\text { Current Data } & \text { Parameters } \\ \text { NAME } & \mathrm{XZ}-3-21 \\ \text { EXPNO } & 1 \\ \text { PROCNO } & 1\end{array}$

F2 - Acquisition Parameters Date__ 20200618

Time- $13.13 \mathrm{~h}$

INSTRU

PROBHD

PULPROG

spect

SOLVENT

NS

DS

SWH

FIDRES

$A Q$

RG

DW

DE

TE

D 1 0

$\mathrm{SFO1}$

NUC1

PO

P 1
PLW1
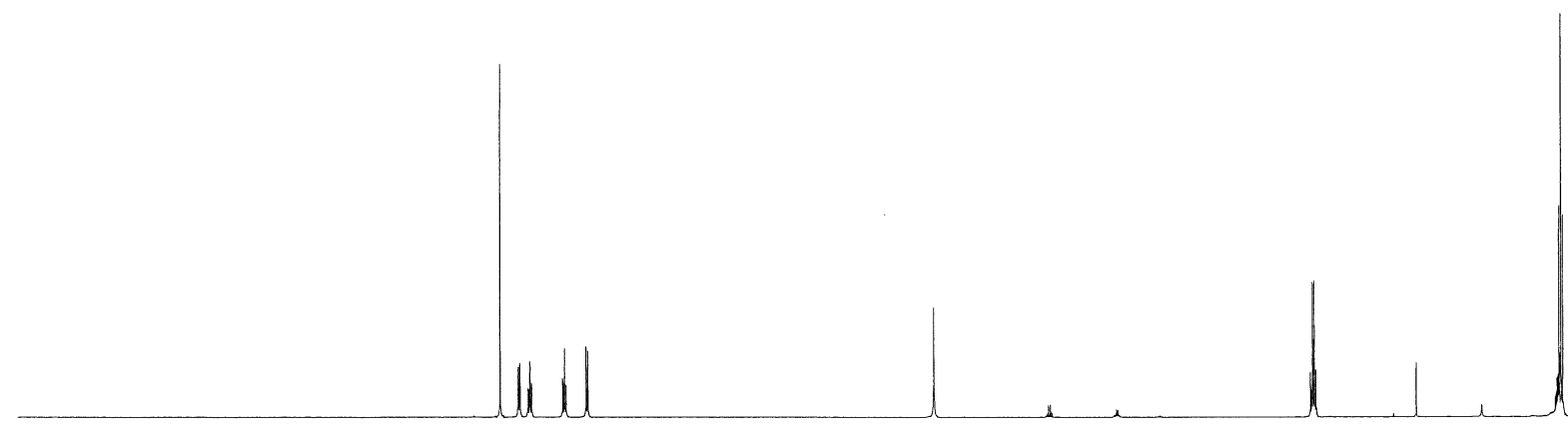

zg30
65536

CDCl3

16
16

$10504.202 \mathrm{~Hz}$

$0.320563 \mathrm{~Hz}$

$3.1195135 \mathrm{sec}$ 22.6

47.600 usec 20.00 usec

$303.2 \mathrm{~K}$

$1.00000000 \mathrm{sec}$

$1.00000000 \mathrm{sec}$

$3 \mathrm{H}$ $1 \mathrm{H}$
$3.17 \mathrm{usec}$ 3.17 usec
9.50 usec

F2 - Processing parameters SI 131072

SF $\quad 700.0070166 \mathrm{MHz}$

WDW

WDW

LB

$\mathrm{PC}$

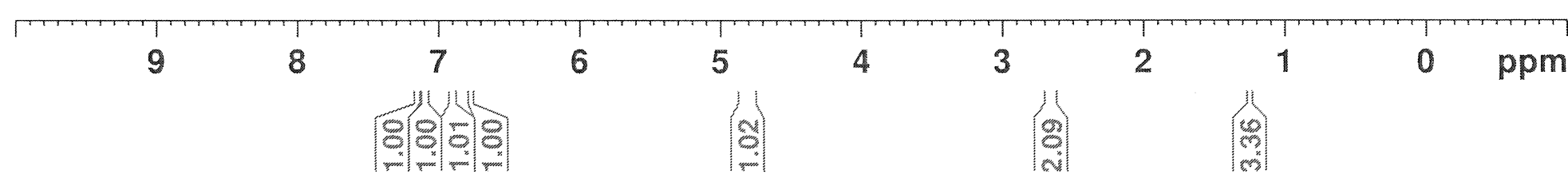




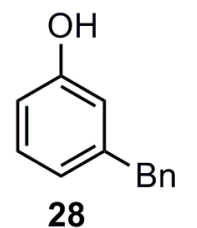

Current Data Parameters

F2 - Acquisition Parameters

Date__ 20200622
Time
$15.57 \mathrm{~h}$

28

INSTRUM

PROBHD

PULPROG

z151340 spect

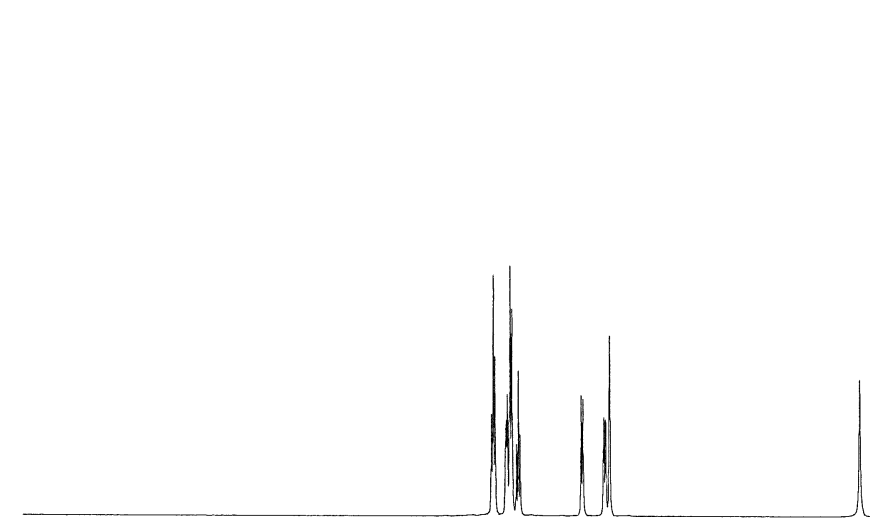

F2 - Processing parameters

\begin{tabular}{|c|c|c|c|c|c|c|c|c|}
\hline 9 & 8 & 5 & 4 & 3 & 2 & 1 & 0 & pom \\
\hline
\end{tabular}


<smiles>Oc1ccccc1Br</smiles>

29
Current Data Parameters $\mathrm{XZ}-2-296$ PROCNO

F2 - Acquisition Parameters Date__ 20200622 Time $\quad 15.48 \mathrm{~h}$ Z151340 spect PUIPROG $\mathrm{zg} 30$
65536 TD $\quad 65536$ NS NS

DS

SWH $0.320563 \mathrm{~Hz}$ $3.1195135 \mathrm{sec}$ 20.2 47.600 usec $303.2 \mathrm{~K}$ $1.00000000 \mathrm{sec}$ $700.0115500 \mathrm{MHz}$ $1 \mathrm{H}$ 3.17 usec 9.50 usec PLW1 $13.00000000 \mathrm{~W}$

F2 - Processing parameters SI 131072 SF $\quad 700.0070181 \mathrm{MHz}$

$\Gamma$ 


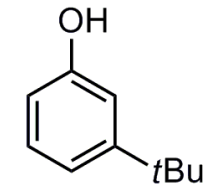

30
Current Data Parameters

NAME

EXPNO

$\mathrm{Xz}-2-248-2$

PROCNO

F2 - Acquisition Parameter Date__ 20181001

Time $21.55 \mathrm{~h}$

INSTRUM spect

PROBHD Z151340_0001

PULPROG zg30

TD

SOLVENT

DS

SWH

FIDRES

AQ

RG

DW

DE

D 1
TD 0
SFO1

TDO

$\mathrm{SFO1}$

(1)

P LW1

CDC 13

$10504.202 \mathrm{~Hz}$

$0.320563 \mathrm{~Hz}$

$3.1195135 \mathrm{sec}$

5

47.600 usec

10.00 usec

$303.2 \mathrm{~K}$

$1.00000000 \mathrm{sec}$

1

$1 \mathrm{H}$

11.00 usec

$13.00000000 \mathrm{~W}$

F2 - Processing parameters

SI $\quad 131072$

SF $\quad 700.0070180 \mathrm{MHz}$

WDW

SSB 0

$\mathrm{Hz}$

1.00

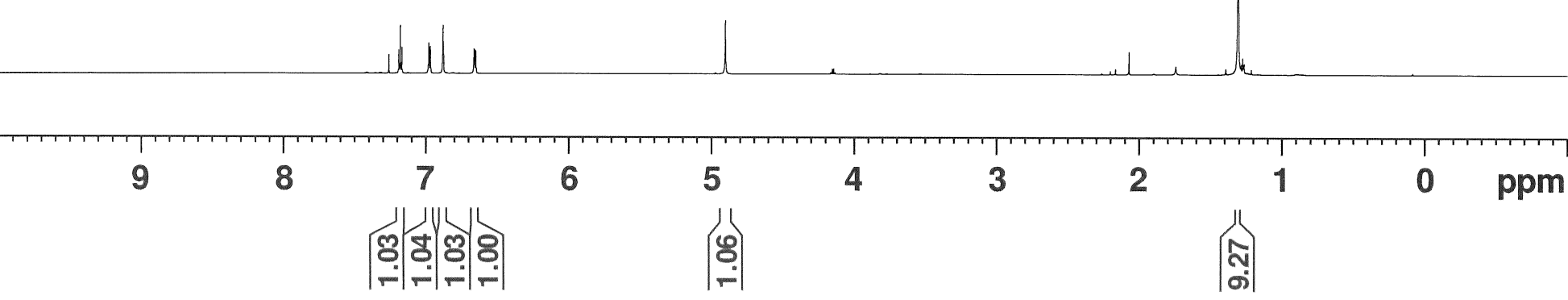




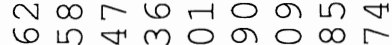

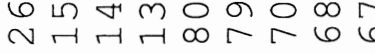
- -10

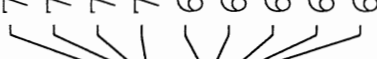
$\rightarrow$

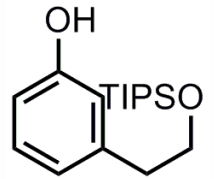

31

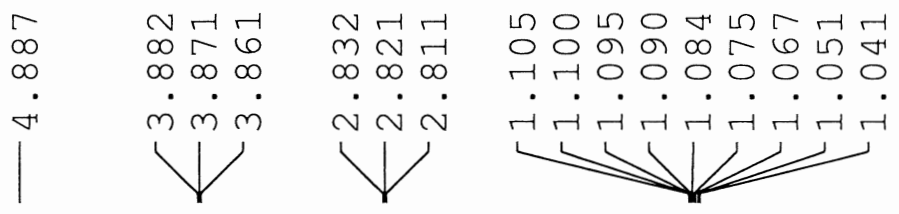

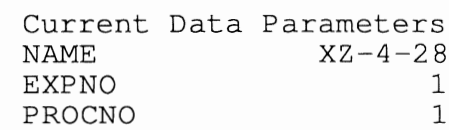

2 - Acquisition Parameter

Date_ 20191007

Time $\quad 13.31 \mathrm{~h}$

spect

PROBHD Z151340 0001

PUIPROG_ zg30

SOLVENT

SWH

$0.320563 \mathrm{~Hz}$

AQ

TE

TDO
SFO1
NUC1

$\mathrm{PO}$

$\mathrm{P} 1$

PLW1

$13.00000000 \mathrm{~W}$

F2 - Processing parameters

SI

SF $\quad 700.0070155 \mathrm{MHz}$

SSB

$\mathrm{LB}$

GB

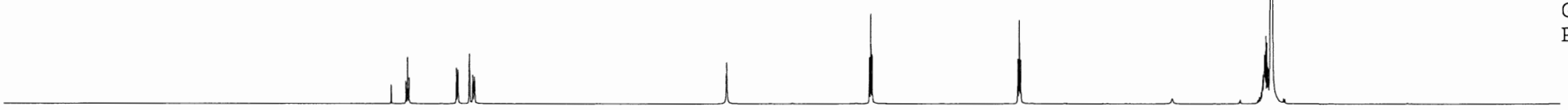

1.00

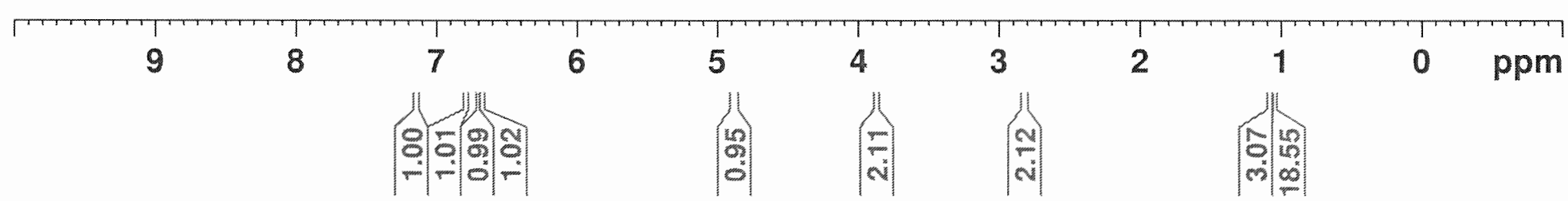


<smiles>Oc1cccc2c1CCC2</smiles>

31
Current Data Parameters NAME

EXPNO

PROCNO

F2 - Acquisition Parameters

Date_ 20191007

Time

13.37

NSTRUM

PROBHD

PULPROG

spect

SOLVENT

NS

DS

SWH

FIDRES

$A Q$
$R G$

RG
DW
DE

DE

(1)

D1

TD 0

$\mathrm{SFO}$

NUC1

$\mathrm{PO}$

$\mathrm{P} 1$

PLW1

$\mathrm{SFO} 2$

NUC2

CPDPRG [ 2

PCPD 2

PIW2

PLW12

PLW13

zgpg 30

65536

$\mathrm{CDCl} 3$
8

4
$42613.637 \mathrm{~Hz}$

$1.300465 \mathrm{~Hz}$

$0.7689557 \mathrm{sec}$

11.733 usec

18.00 usec$$
303.2 \mathrm{~K}
$$

$2.00000000 \mathrm{sec}$

$0.03000000 \mathrm{sec}$

$176.0362620 \mathrm{MHz}$ $13 \mathrm{C}$

3.75 use 1.25 use

$29.00000000 \mathrm{~W}$ $700.0098000 \mathrm{MHz}$ $1 \mathrm{H}$
waltz 16 65.00 usec

$13.00000000 \mathrm{~W}$ $0.27768999 \mathrm{~W}$ $0.13982999 \mathrm{~W}$

2 - Processing parameters

SI 131072

SF $\quad 176.0169002 \mathrm{MHz}$

$\mathrm{SSB}$

GB

\begin{tabular}{|c|c|c|c|c|c|c|c|c|c|}
\hline 200 & 180 & 160 & 140 & 120 & 100 & 80 & 60 & $\begin{array}{c}1 \\
40\end{array}$ & $\begin{array}{c}1 \\
20\end{array}$ \\
\hline
\end{tabular}


N

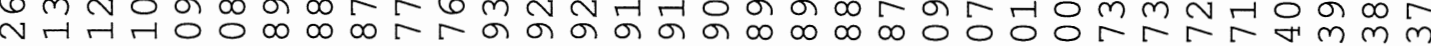

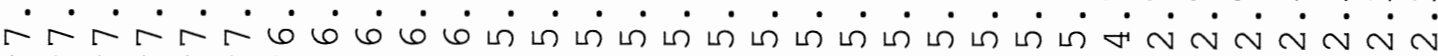

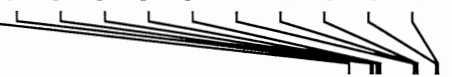

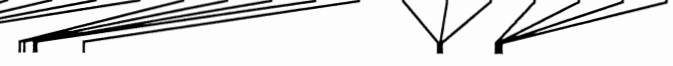

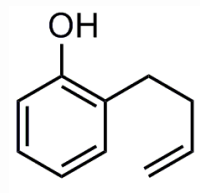

32

Current Data Parameter

F2 - Acquisition Parameter

Date__ 20191018

Time $10.17 \mathrm{~h}$

INSTRUM spect

PROBHD Z151340_0001

PULPROG zg30

TD 65536

SOLVENT CDCl3

16

$D S$

FIDRES

FIDRE

$10504.202 \mathrm{~Hz}$

RG
$D W$

TE

$\mathrm{D} 1$

TEO

$\mathrm{SEO1}$

P 1

$0.320563 \mathrm{~Hz}$

$3.1195135 \mathrm{sec}$ 25.4

47.600 usec

20.00 usec

$303.2 \mathrm{~K}$

$1.00000000 \mathrm{sec}$

100.011550

$1 \mathrm{H}$

3.17 usec

3.17 usec

$13.00000000 \mathrm{~W}$

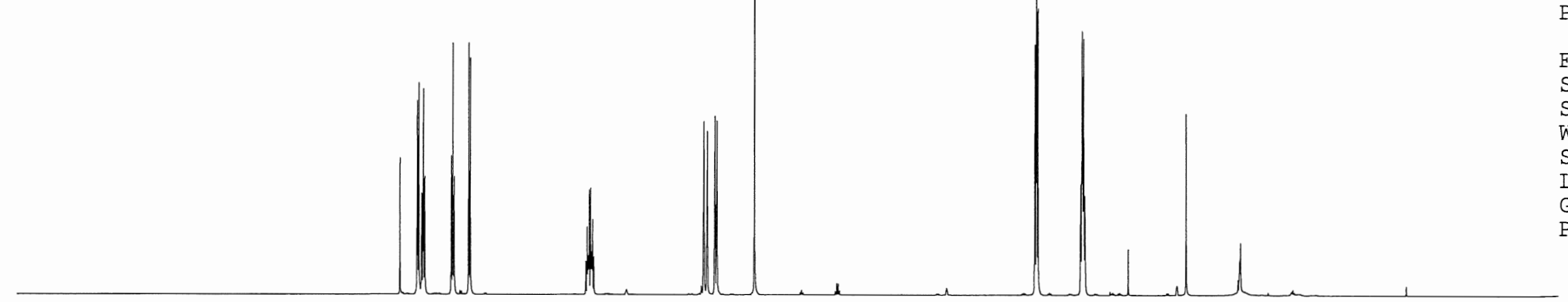

2 - Processing parameters

SI 131072

$700.0070167 \mathrm{MHz}$

no

0
0
0

$\amalg B$

PC

1.00

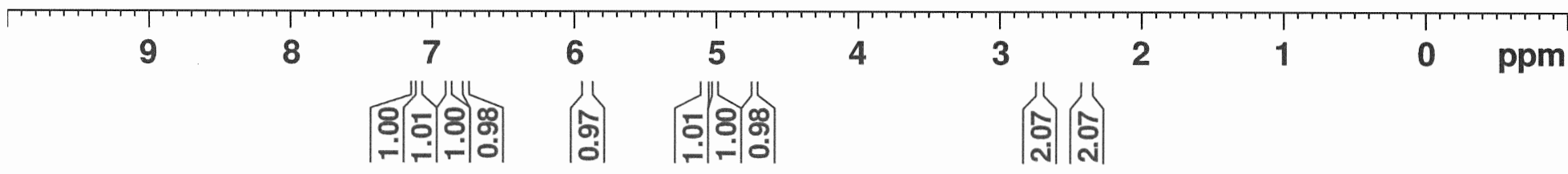




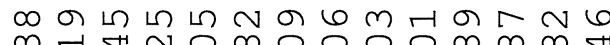
6 다

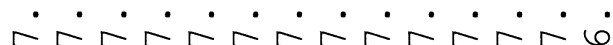

$+2$
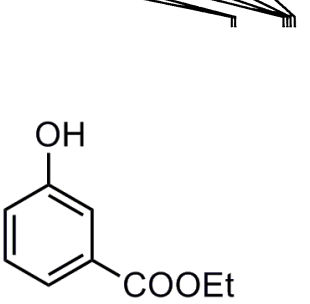

33 m $m \Omega$ अ अ $m$ $\cdot \cdot \cdot \cdot$

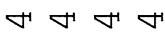

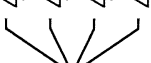

$\infty \circ N$

강

$\cdot \cdot$

तr

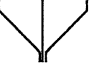

Current Data Parameter $\mathrm{zX}-2-149-2$

EXPNO

PROCNO

1

F2 - Acquisition Parameter Date__ 20180527

Time

13.18

INSTRUM

PROBHD

PULPROG zg30

$5 \mathrm{~mm} P A B$ spect

SOLVENT

NS

DS

SWH

IDRES

RG

DW

DE

TE

D 1

TD 0

$\mathrm{zg} 30$
65536

CDC13

32

$8278.146 \mathrm{~Hz}$

$0.126314 \mathrm{~Hz}$

$3.9583745 \mathrm{sec}$ 90.5

60.400 usec 6.50 usec $303.1 \mathrm{~K}$

$1.00000000 \mathrm{sec}$

NUC 1

P 1

P L 1

PLIW

$\mathrm{SFO1}$

CHANNEL I

$\mathrm{fl}=$

14.00 usec

$0 \mathrm{~dB}$

$10.27361584 \mathrm{~W}$ $400.1424710 \mathrm{MH}$

F2 - Processing parameters SI 131072

SF $\quad 400.1400000 \mathrm{MHz}$

$S S B$

LB

$\mathrm{GB}$
$\mathrm{PC}$

$\mathrm{O} \mathrm{Hz}$

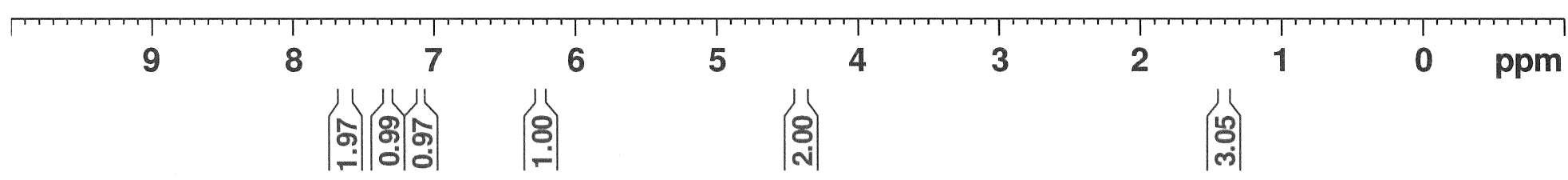


Nom $\infty \circ$ a

NoOO $\mathrm{N}-6$

- $\dot{0} \dot{0} \dot{0} 6 \dot{6}$

$\$$

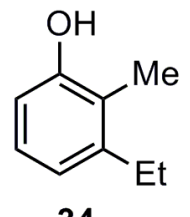

34 $\begin{array}{lll}0 & 0 \\ m & m & 0\end{array}$

$6666 \%$ กั

$\dot{\sim} \sim \dot{\sim}$

Nij

Current Data Parameters

AME

EXPNO

PROCNO

$\mathrm{XZ}-2-287$

F2 - Acquisition Parameters

Date__ 20181105

Time $15.35 \mathrm{~h}$

INSTRUM spect

PROBHD Z151340_0001 (

PULPROG $\quad$ zg30

$\begin{array}{lr}\text { TD } & \text { 2930 } \\ \text { TD } & 65536\end{array}$

SOLVENT CDCI3

NS

DS

SWH

FIDRES

AQ

DW

DE

TE

D1

TDO

NUC1

P1

P 1
PLW1

16
2
10504.202

$0.320563 \mathrm{~Hz}$

$3.1195135 \mathrm{sec}$ 6.35

47.600 usec

10.00 usec

$303.2 \mathrm{~K}$

$1.00000000 \mathrm{sec}$

$700.0115500^{1} \mathrm{MHz}$

$1 \mathrm{H}$
9.50 usec

$13.00000000 \mathrm{~W}$

22 - Processing parameters

SI 131072

$\mathrm{SF}$

WDW

SSB

LB

$\mathrm{PC}$

$700.0070150 \mathrm{MHz}$

0

$\mathrm{O} \mathrm{Hz}$

no

1.00

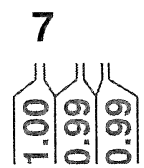

$6 \quad 5$

4

3

人)

$0 \quad \mathrm{ppm}$

5

$\left|\begin{array}{l}\Omega \\ g \\ \text { ले }\end{array}\right|$ 

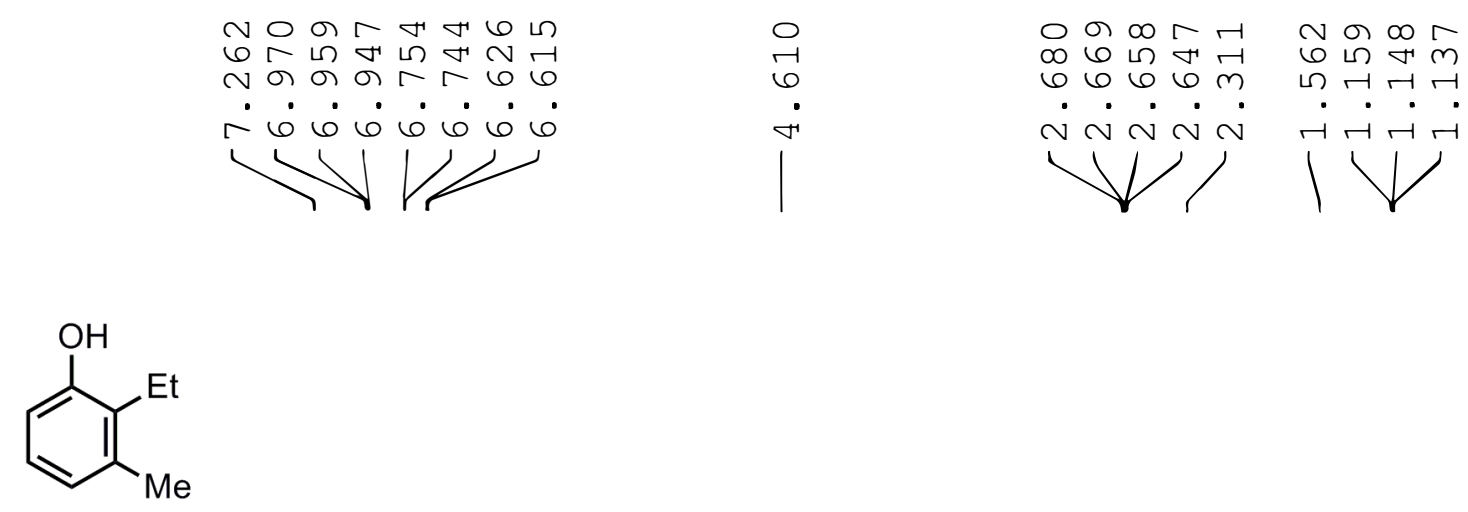

35

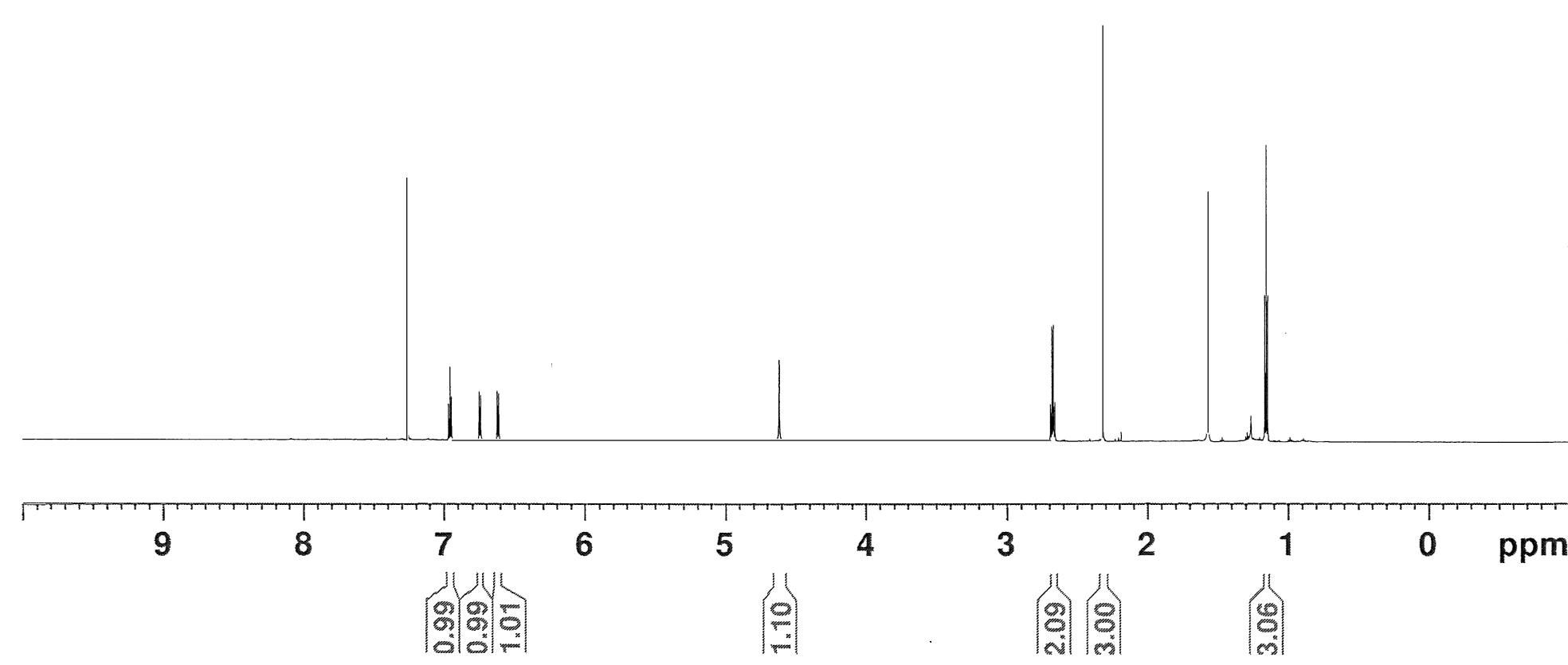

Current Data Parameters 


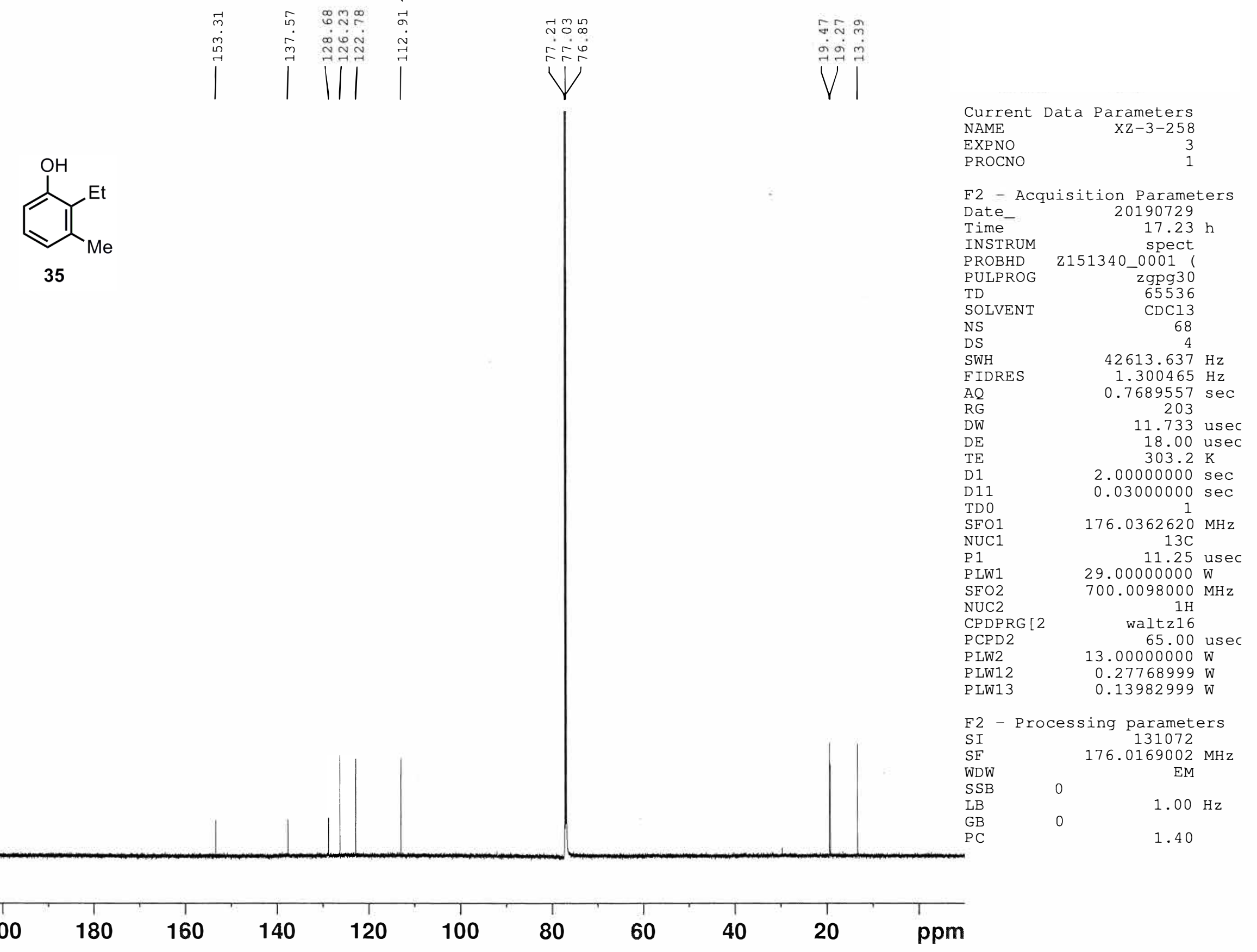



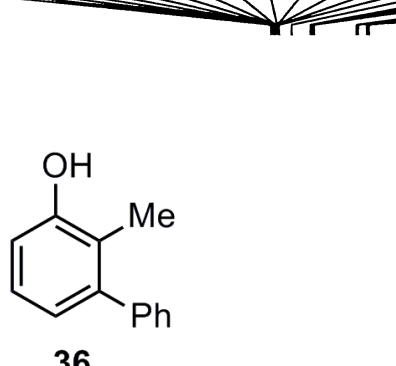

36
Current Data Parameters NAME

EXPNO

PROCNO $\mathrm{XZ}-2-238$

(5)

Time $15.19 \mathrm{~h}$

INSTRUM spect

PROBHD Z151340_0001 (
PULPROG
zg30

TD

SOLVENT

NS

DS

SWH

AQ

RG

$\mathrm{DW}$

$\mathrm{DE}$

TE
D1
TD 0

SFO1

P 1

PLW

2 Processing

Processing parameters

SI 131072

SF $\quad 700.0070177 \mathrm{MHz}$

WDW

SSB

LB

GB

0

$0 \mathrm{~Hz}$

no

$\mathrm{PC}$

1.00

9

8

7

6

5

4

3

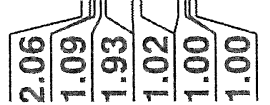

$\left|\begin{array}{c}\mathrm{N} \\ 0\end{array}\right|$

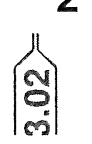

ppm

|⿵冂⿰㐅㐅⿸⿻三丨子 

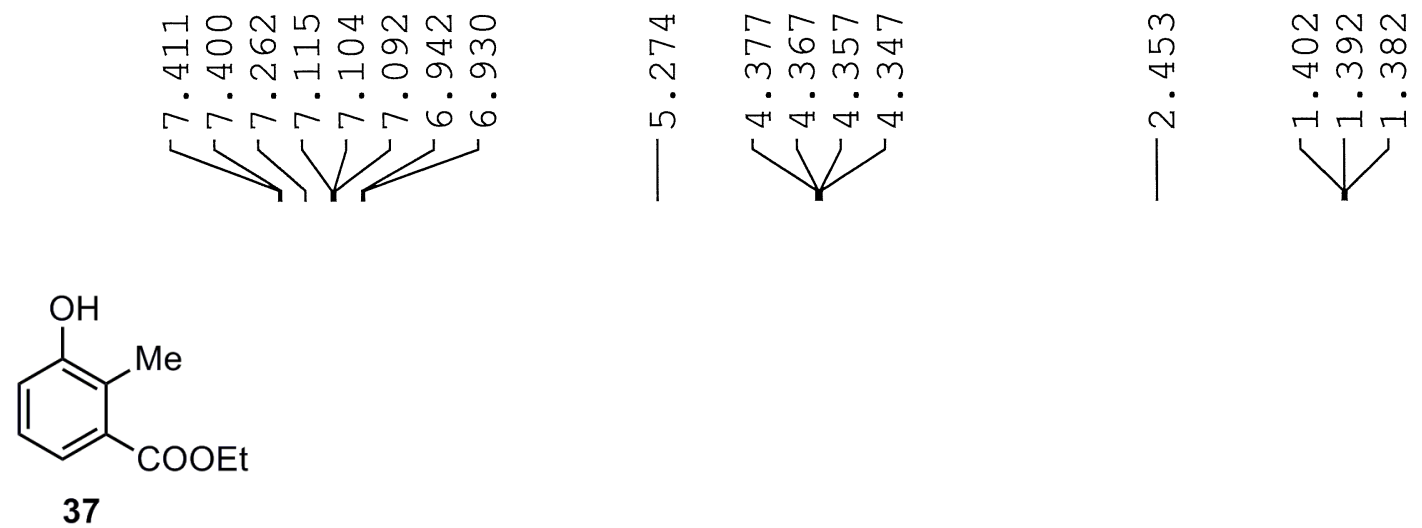
Current Data Parameters
$\mathrm{Xz}-3-3$
ROCNO

F2 - Acquisition Parameter

Date__ 20181214

Time $14.30 \mathrm{~h}$

INSTRUM

PROBHD Z151340_0001

PULPROG zg30

TD 65536

SOLVENT CDCl3

DS

SWH

FIDRES

16

$\mathrm{AQ}$

$10504.202 \mathrm{~Hz}$

$0.320563 \mathrm{~Hz}$

$3.1195135 \mathrm{sec}$ 6.35

47.600 usec 10.00 usec

$1.00000000 \mathrm{sec}$

11000

P 1

$$
1 \mathrm{H}
$$

LW1 $13.00000000 \mathrm{~W}$

F 2 - Processing parameters

SI 131072

SF $\quad 700.0070159 \mathrm{MHz}$

NDW

S S B

LB
GB
PC

no

0
0 $\mathrm{~Hz}$

1.00
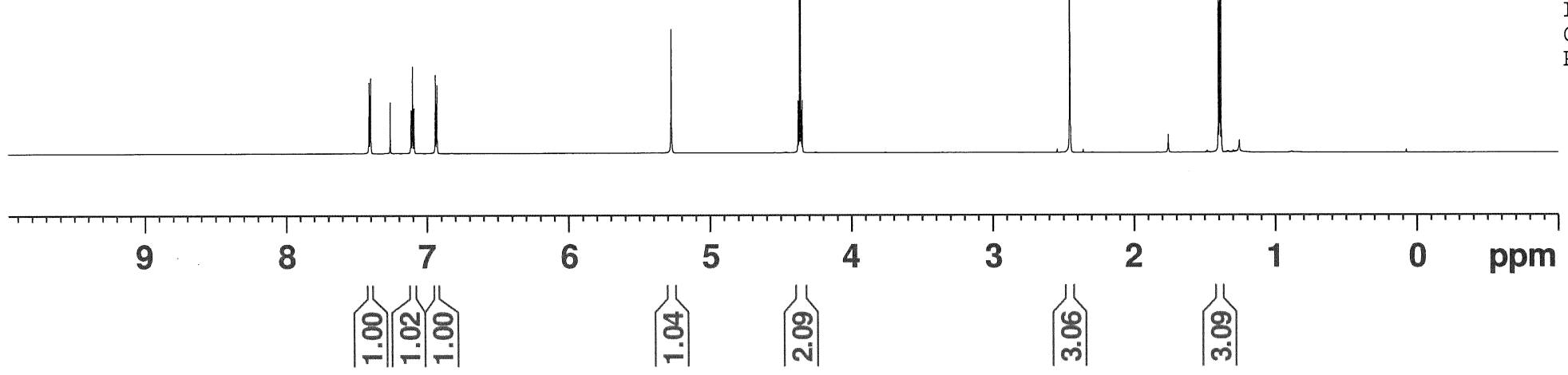
$m \sim \sim m \sim \not \infty \infty$ ก 6 으 $\mathrm{N}$ mm $\mathrm{m}$ त० व in isisis
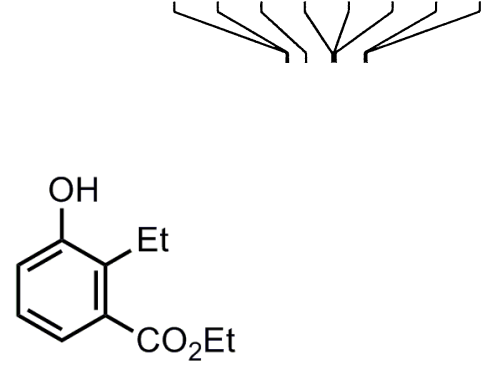

38

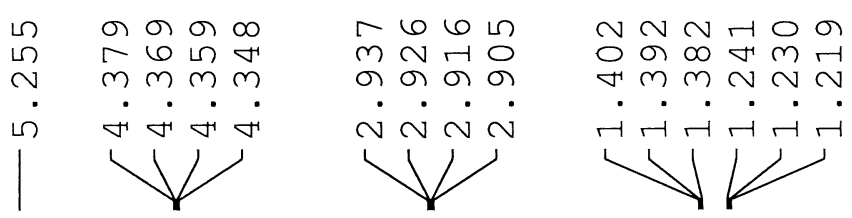
$\begin{array}{ll}\text { Current Data } & \text { Parameters } \\ \text { NAME } & \text { XZ-3-127-2 }\end{array}$

EXPNO

PROCNO

F2 - Acquisition Parameters

Date_ 20190408

Time

INSTRUM

PROBHD

TD

SOLVENT

NS

DS

SWH

FIDRES

AQ
RG
DW

DW

TE

D1

TDO

$\mathrm{SFO1}$

NUC1

P 1

z151340_0001)

$\mathrm{zg} 30$
65536

2
$10504.202 \mathrm{~Hz}$

$0.320563 \mathrm{~Hz}$

$3.1195135 \mathrm{sec}$ 6.35

47.600 usec

10.00 usec

$10.00 \mathrm{us}$
$303.2 \mathrm{~K}$

$1.00000000 \mathrm{sec}$

1.00000000

$1 \mathrm{H}$

$1 \mathrm{H}$
50 use

$13.00000000 \mathrm{~W}$

F2 - Processing parameters

SI 131072

SF $\quad 700.0070166 \mathrm{MHz}$

WDW

$S S B$

LB

0

$0 \mathrm{~Hz}$

1.00

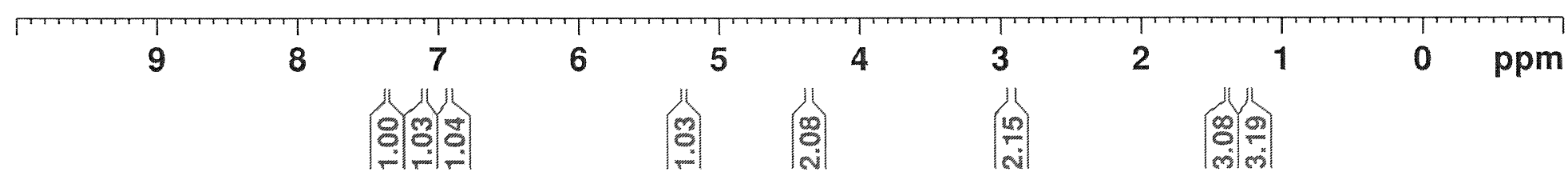




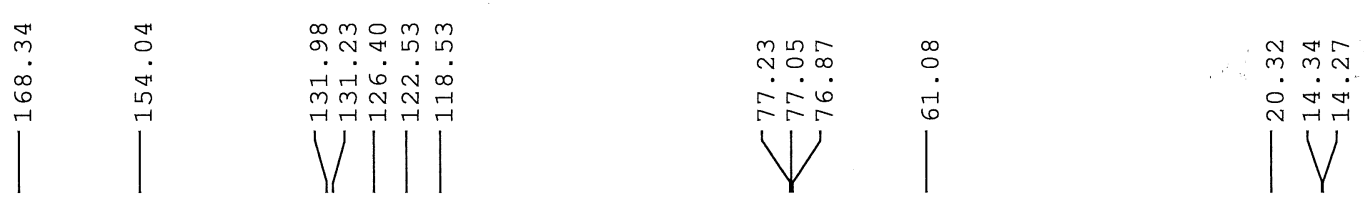

Current Data Parameters

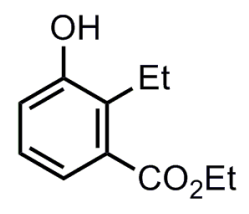

38
PROCNO

F2 - Acquisition Parameter Date__ 20190408

Time

INSTRUM

PROBHD

PULPROG

16.51

Z151340 0001

TD

SOLVENT

NS

D S

FIDRE

$\mathrm{AQ}$

RG

DW

DE

D 1

D 11

$\mathrm{SFO1}$

(1)

P 1

PLW1

$\mathrm{SFO} 2$

$\mathrm{NUC} 2$

CPDPRG [ 2

PCPD 2

P LW2

P LW1 2

PLW1 3

z gpg30

18

$42613.637 \mathrm{~Hz}$

1.300465 Hz $0.7689557 \mathrm{sec}$ 203

11.733 usec 18.00 use $303.2 \mathrm{~K}$

$2.00000000 \mathrm{sec}$ $0.03000000 \mathrm{sec}$

$176.0362620 \mathrm{MHz}$ $13 \mathrm{C}$

1.25 usec $29.00000000 \mathrm{~W}$ $700.0098000 \mathrm{MHz}$

$$
\begin{gathered}
1 \mathrm{H} \\
\text { waltz } 16
\end{gathered}
$$
65.00 user $13.00000000 \mathrm{~W}$ 0.27768999 $0.13982999 \mathrm{~W}$ SI 131072 SF $\quad 176.0169002 \mathrm{MHz}$ WDW EM

SSB

LB

$\mathrm{GB}$
$\mathrm{PC}$

0

$1.00 \mathrm{~Hz}$

1.40

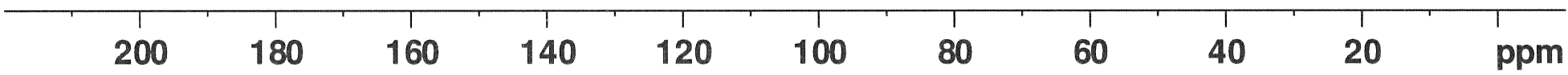




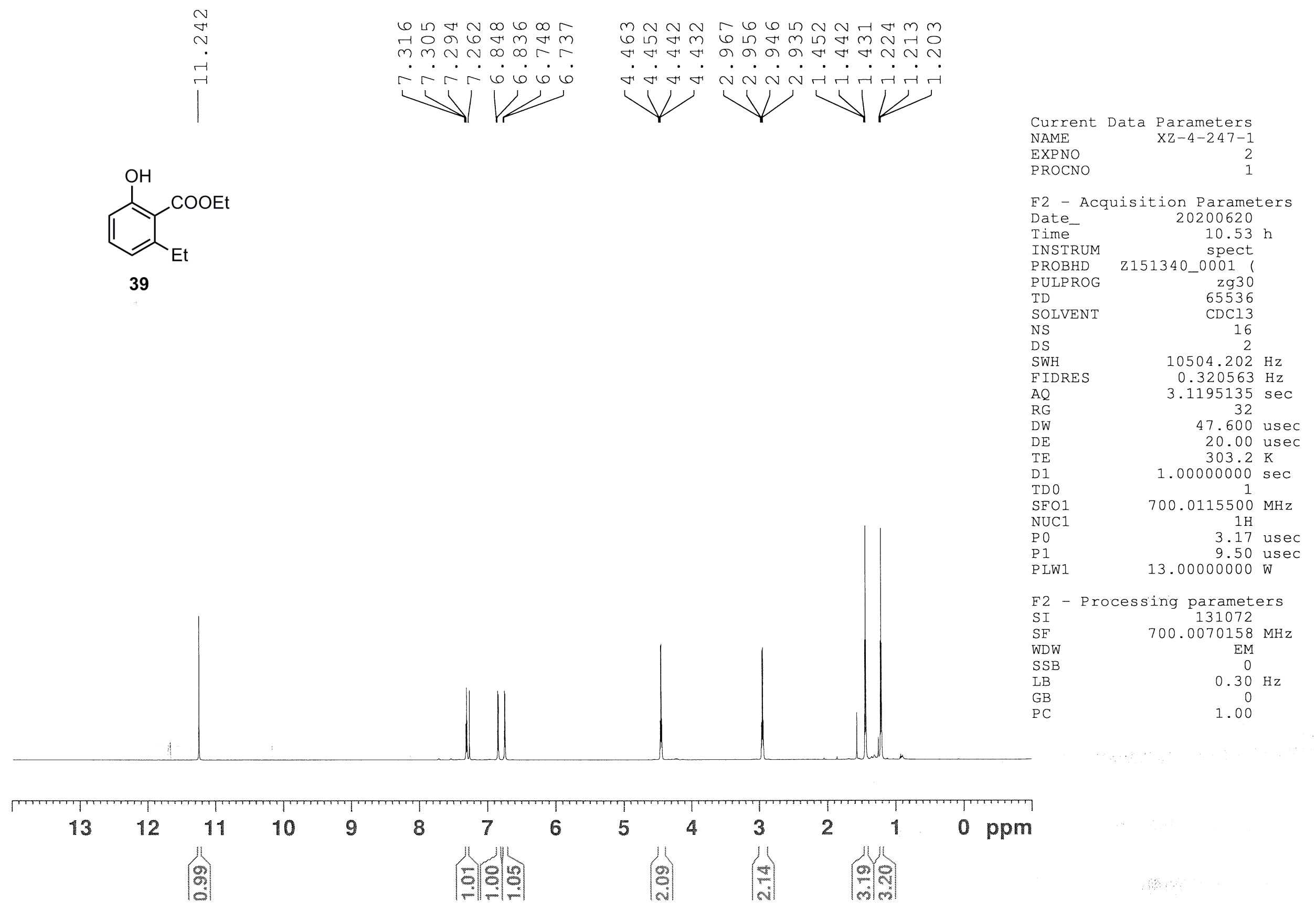




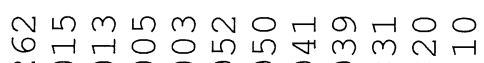

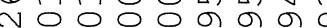
-

$\longrightarrow$

ก म

$\dot{\sim} \dot{\sim} \dot{\sim} \dot{\sim} \dot{\sim} \dot{\dot{H}} \dot{\sim} \dot{-} \dot{0} \dot{0}$
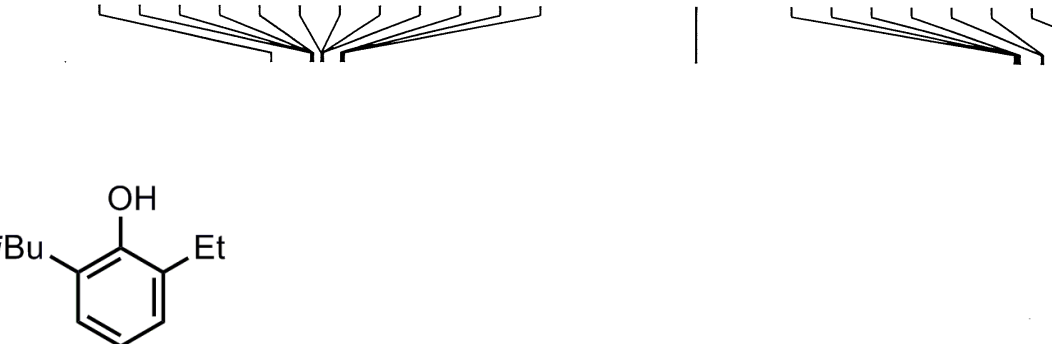

40
Current Data Parameters

$\mathrm{XZ}-3-100$

PROCNO

F2 - Acquisition Parameters

Date_ 20190312

Time

INSTRUM

PROBHD

PULPROG

TD

DS

SWH

AQ

RG

DW

DE

TE

TDO

SFO1

P1

2151340_0001 (

65536$$
\begin{array}{r}
16 \\
2
\end{array}
$$

$10504.202 \mathrm{~Hz}$

$0.320563 \mathrm{~Hz}$

$3.1195135 \mathrm{sec}$ 5.6

47.600 usec 10.00 usec $1.00000000 \mathrm{~K}$ $1.00000000 \mathrm{sec}$ $700.0115500 \mathrm{MHz}$

$1 \mathrm{H}$
9.50 usec $13.00000000 \mathrm{w}$

F2 - Processing parameters

SI $\quad 131072$

$7 \mathrm{MHz}$

WDW

SSB

LB

no

$\mathrm{O} \mathrm{Hz}$

0
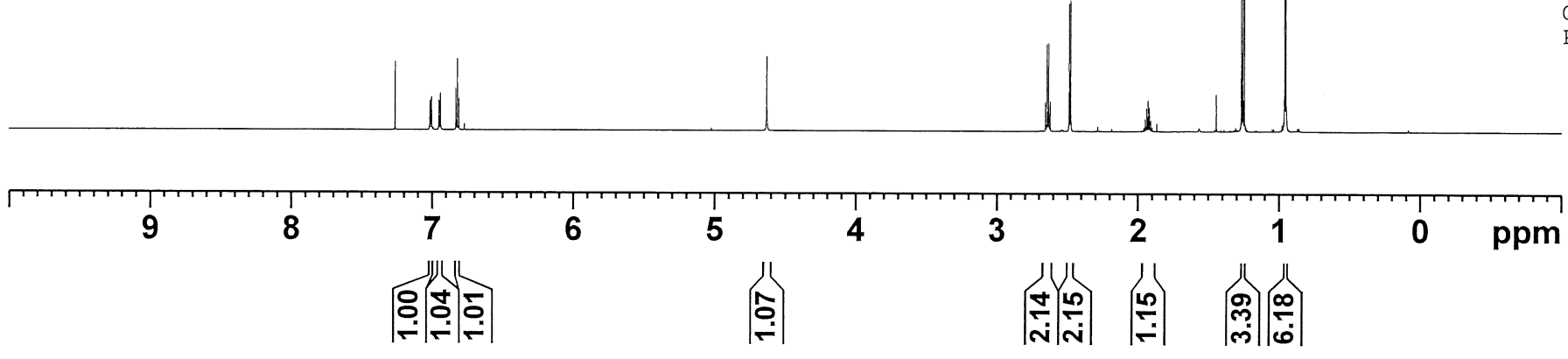


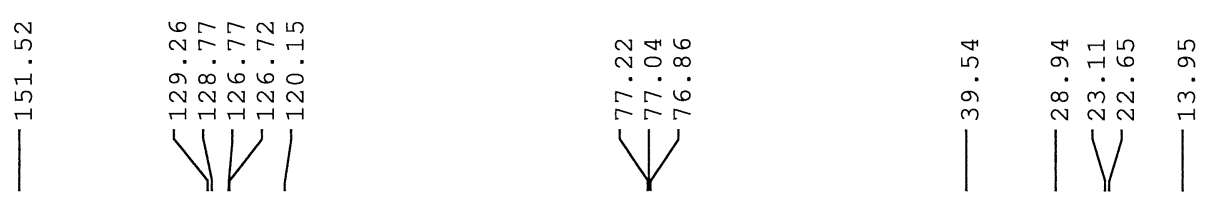

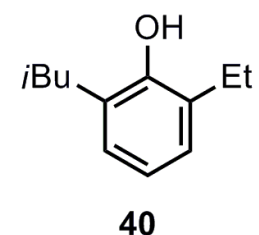

Current Data Parameters

F2 - Acquisition Parameter Date__ 20190312

Time $\quad 10.01 \mathrm{~h}$

INSTRUM spect

40

ROBHD

PULPROG

Z151340_0001

DS

SWH

FIDRES

$\mathrm{AQ}$

RG

$\mathrm{DW}$

$\mathrm{DE}$

D 1
D 11
TD 0

SF O1

$\mathrm{P} 1$

P LW1

$\mathrm{SFO} 2$

NUC 2

CPDPRG [ 2

P CP D 2

P LW2

PLW12

PLW1 3

F2 - Processing parameters

SI 131072

176.0169002 MHz

SF

WDW

$S S B$

LB

$\mathrm{GB}$
$\mathrm{PC}$
$42613.637 \mathrm{~Hz}$

$1.300465 \mathrm{~Hz}$

$0.7689557 \mathrm{sec}$

11.733 usec 18.00 usec $303.2 \mathrm{~K}$

$2.00000000 \mathrm{sec}$ $0.03000000 \mathrm{sec}$

$176.0362620 \mathrm{MHz}$ $13 \mathrm{C}$

$29.00000000 \mathrm{w}$ $700.0098000 \mathrm{MH}$$$
\begin{gathered}
1 \mathrm{H} \\
\text { waltz } 16
\end{gathered}
$$

65.00 use

$13.00000000 \mathrm{~W}$

$0.27768999 \mathrm{~W}$

$0.13982999 \mathrm{~W}$
0

$1.00 \mathrm{~Hz}$

1.40

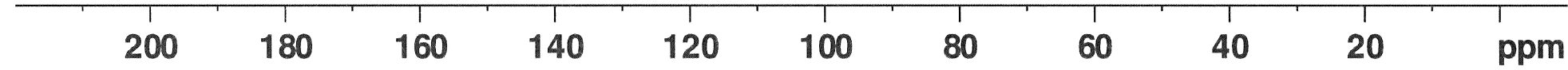




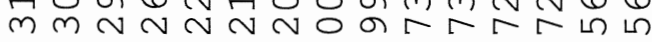
rirrarar $\stackrel{2}{\longrightarrow}$

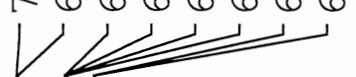

ํㅟำ
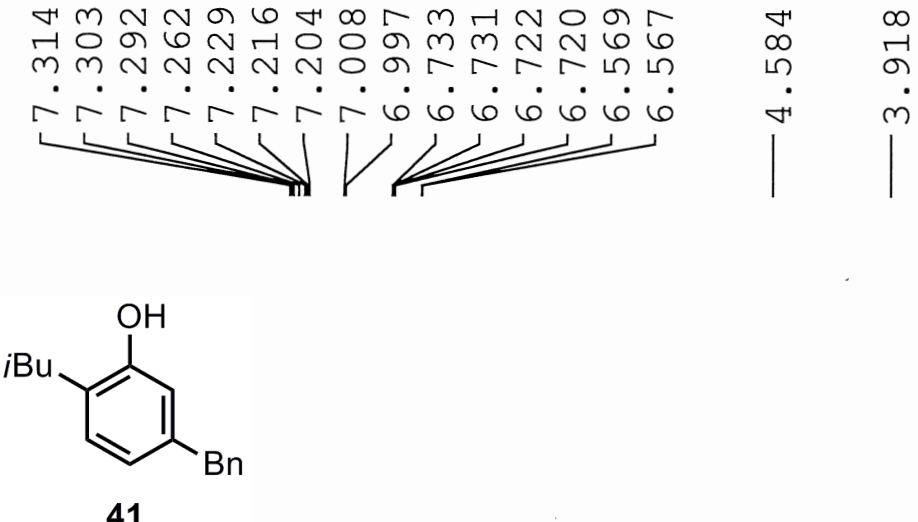

ஸा अ

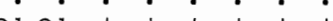

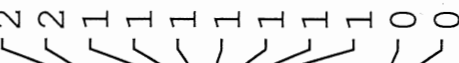

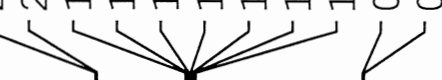

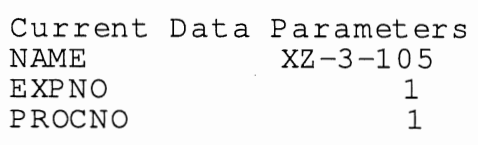

PROCNO

F2 - Acquisition Parameter Date__ 20190326

Time

INSTRUM

PROBHD

PULPROG

$13.27 \mathrm{~h}$

Z151340_0001

$P$

SOLVENT

NS

DS

FIDRES

$\mathrm{AQ}$

RG

DW

$\mathrm{DE}$

TD 0

$\mathrm{SFO1}$

NUC 1

$\mathrm{P} 1$

P LW1

$13.00000000 \mathrm{~W}$

SI 131072

SF $\quad 700.0070163 \mathrm{MHz}$

WDW

LB

EM

$0.30 \mathrm{~Hz}$

1.00
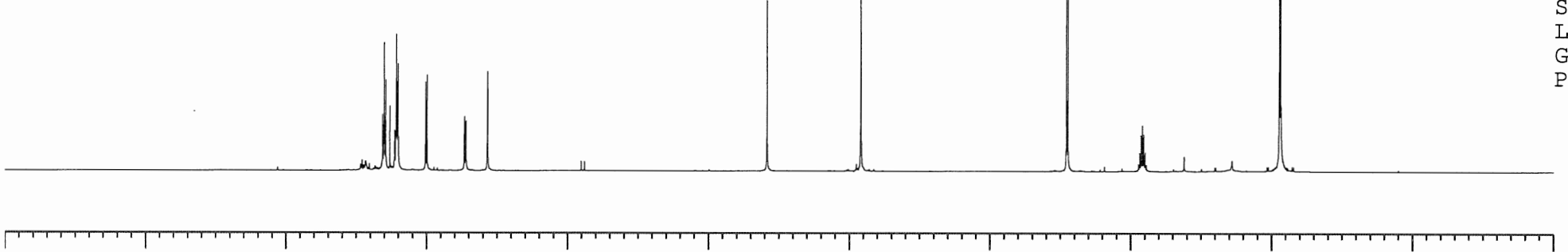

6

5 


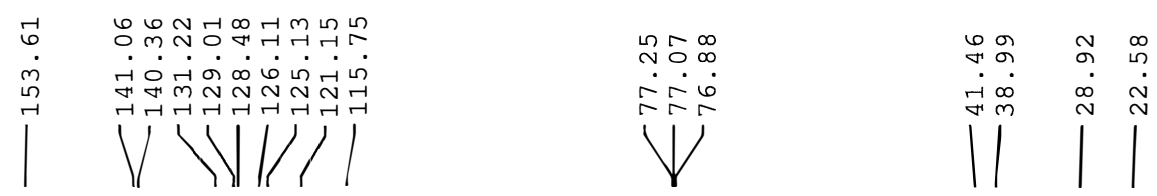

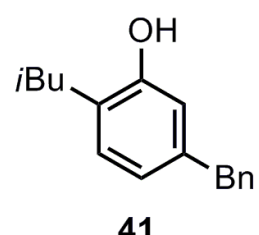
Current Data Parameters NAME
EXPNO
PROCNO

F2 - Acquisition Parameter

Date_ 20190326

Time $13.33 \mathrm{~h}$

INSTRUM

PROBHD Z151340_0001

z gpg30

TD

SOLVENT

NS

DS

FIDRES

$\mathrm{AQ}$

RG

DW

$\mathrm{DE}$

TE

D 11

DI1

SF 01

NUC 1

P 1

PLW1

$\mathrm{SF} O 2$

NUC2

CPDPRG [ 2

P CP D 2

P LW2

P LW1 2

65536

$\mathrm{CDCl} 3$ 26

$42613.637 \mathrm{~Hz}$ $1.300465 \mathrm{~Hz}$ $0.7689557 \mathrm{sec}$ 203

11.733 usec 18.00 usec $303.2 \mathrm{~K}$

$2.00000000 \mathrm{sec}$ $0.03000000 \mathrm{sec}$ 1 $176.0362620 \mathrm{MHz}$ $13 \mathrm{C}$

1.25 usec $29.00000000 \mathrm{~W}$
$700.0098000 \mathrm{MHz}$

$$
\begin{gathered}
1 \mathrm{H} \\
\text { waltz } 16
\end{gathered}
$$

65.00 user $13.00000000 \mathrm{~W}$ $0.27768999 \mathrm{~W}$

PLW13

$0.13982999 \mathrm{~W}$

F2 - Processing parameters SI 131072 SF $\quad 176.0169002 \mathrm{MHz}$

WDW $\mathrm{EM}$

SSB

LB

PB

$1.00 \mathrm{~Hz}$

1.40

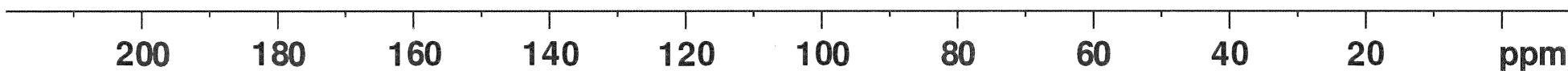




\begin{tabular}{|c|c|c|c|}
\hline N & $\sigma$ & $m \sim r-m$ & $\forall m \sim$ \\
\hline 6 in m r & $m$ & 0 a $\infty \sim m$ & $m \sim H$ \\
\hline No 0 . & r. & 6 는 & $\sim \sim N$ \\
\hline$-r-666$ & $\sigma$ & 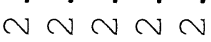 & $\vec{H}-\overrightarrow{1}$ \\
\hline
\end{tabular}

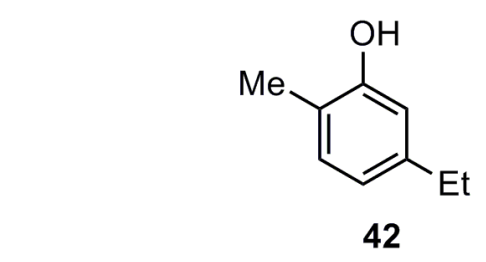

$\begin{array}{lr}\text { Current Data } & \text { Parameters } \\ \text { NAME } & \mathrm{XZ}-3-98 \\ \text { EXPNO } & 4 \\ \text { PROCNO } & 1\end{array}$

F2 - Acquisition Parameters

Date__ 20200622

Time $\quad 16.05$ h

INSTRUM spect

PROBHD Z151340_0001

PULPROG Zg30

$\begin{array}{lr}\text { PULPROG } & \text { Zg30 } \\ \text { TD } & 65536\end{array}$

SOLVENT CDC13

NS

DS

SWH

FIDRES

$A Q$

DW

DE

TE

D1

TDO

$\mathrm{SFO} 1$
NUC1

Po

P 1

PLW1

2
$10504.202 \mathrm{~Hz}$

$0.320563 \mathrm{~Hz}$

$5135 \mathrm{sec}$

20.2

47.600 usec

20.00 usec

$1.00000000 \mathrm{sec}$

$1.00000000 \mathrm{sec}$

$700.0115500 \mathrm{MHz}$

3.17 use

$13.00000000 \mathrm{~W}$

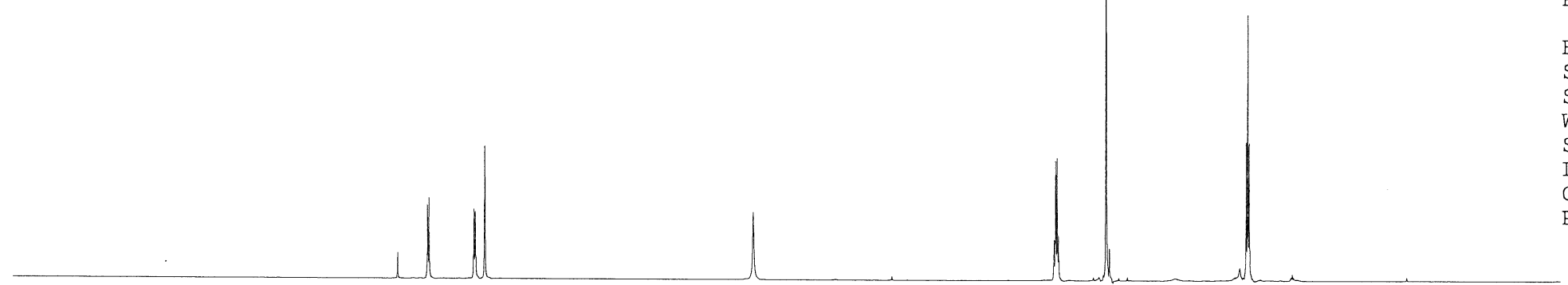

2 - Processing parameters

SI 131072

SF $\quad 700.0070154 \mathrm{MHz}$

WDW

SSB

$\triangle B$

$\mathrm{PC}$

EM
0
$0.30 . \mathrm{Hz}$
0
1.00

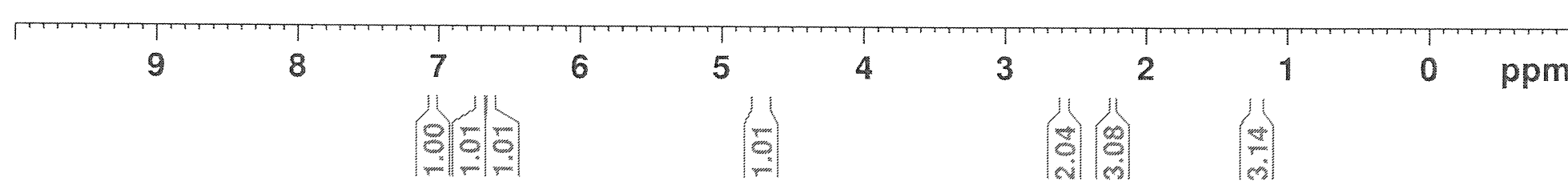




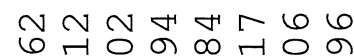

깅 ब

-

3<smiles>CCc1cccc(C)c1O</smiles>

43

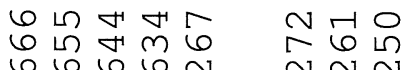

ก.

$\sim \sim \dot{\sim} \sim$

팍

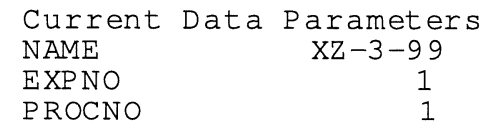

F2 - Acquisition Parameter Date__ 20190311 Time $16.21 \mathrm{~h}$ spect PROBHD Z151340_0001 PULPROG zg30

TD

SOLVENT

NS

DS

SWH

FIDRES

AQ
RG
DW

$\mathrm{DE}$

TE

D1

TD 0

$\mathrm{SFO1}$

SUC

P 1
P LW1

F2 - Processing parameters $\mathrm{SI}$

SF

WDW

$S S B$
$L B$

$\mathrm{LB}$
$\mathrm{GB}$
$\mathrm{PC}$

131072

CDCl3

16

$10504.202 \mathrm{~Hz}$

$0.160281 \mathrm{~Hz}$ $6.2390270 \mathrm{sec}$ 5.6

47.600 usec 10.00 usec $323.2 \mathrm{~K}$

$1.00000000 \mathrm{sec}$ 1 $1 \mathrm{H}$

9.50 usec

$13.00000000 \mathrm{~W}$

131072

$700.0070156 \mathrm{MHz}$ 0

$\mathrm{O} \mathrm{Hz}$

1.00
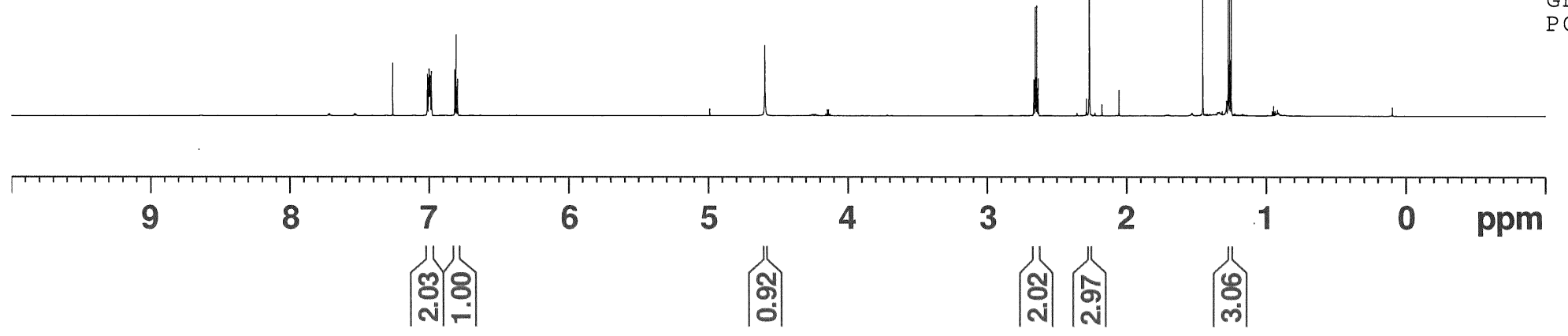
- 6 에 $N$ N

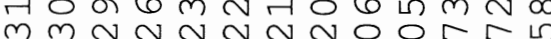

rararararaber

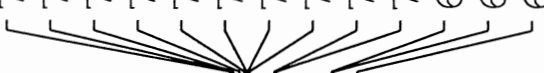

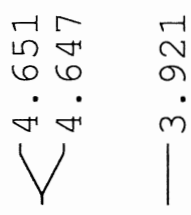

$\infty$

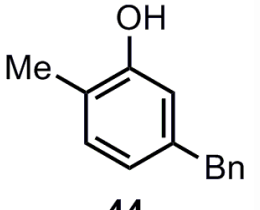
$\mathrm{xz}-3-104$

F2 - Acquisition Parameters

Date_ 20190326

Time $13.14 \mathrm{~h}$

INSTRUM spect

PROBHD Z151340 0001

zg30
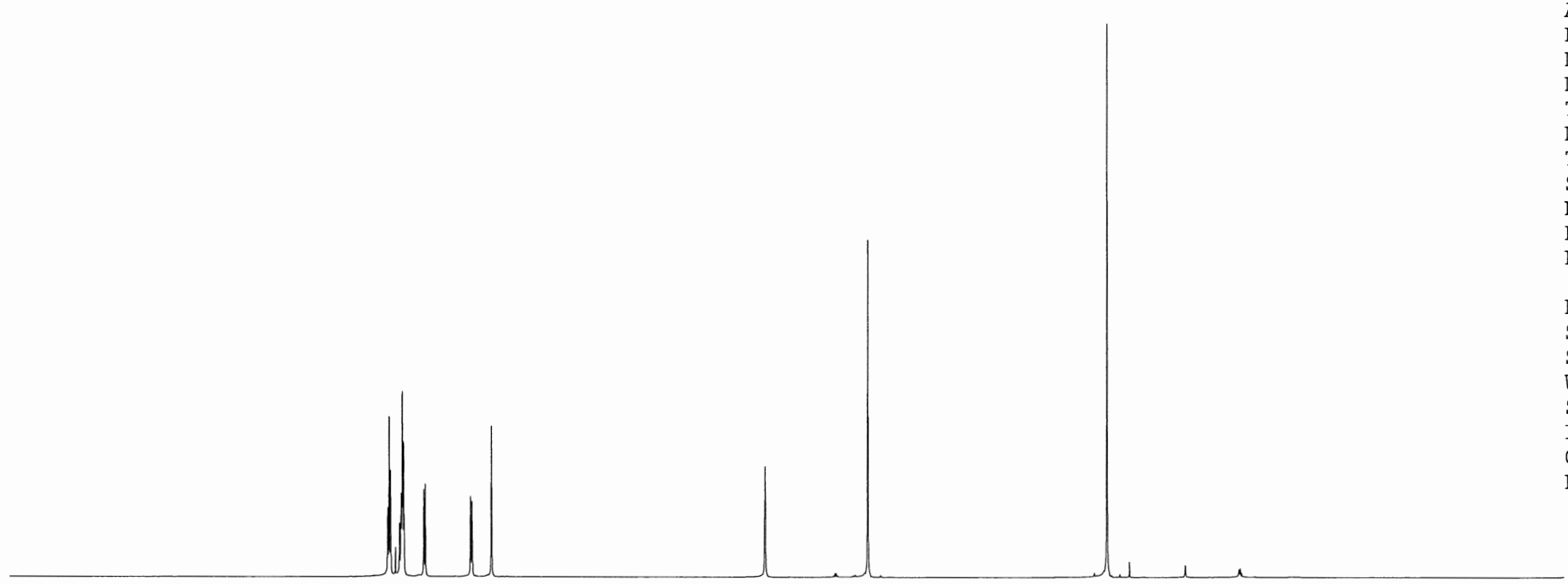

DE

D1

TDO

SFO1

NUC1

P1

zg30
65536

16

$10504.202 \mathrm{~Hz}$

$0.320563 \mathrm{~Hz}$

$3.1195135 \mathrm{sec}$ 6.35

47.600 usec

10.00 usec

$10.00 \mathrm{usec}$

$1.00000000 \mathrm{sec}$

1.00000000

$13.00000000 \mathrm{~W}$

2 - Processing parameter

131072

SF $\quad 700.0070160 \mathrm{MHz}$

WDW no

SSB 0

$\mathrm{LB} \quad \mathrm{OHz}$

PC

1.00

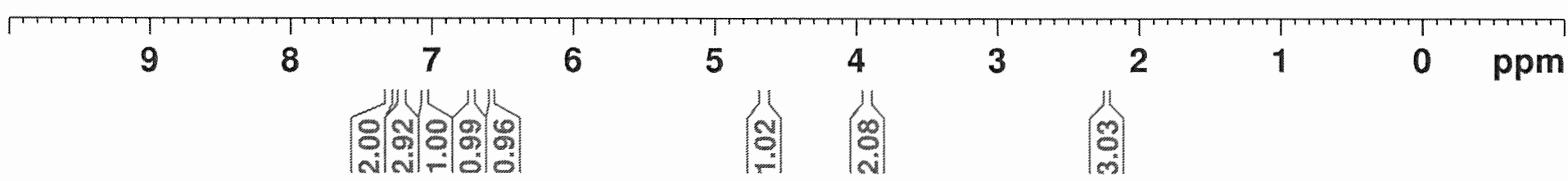




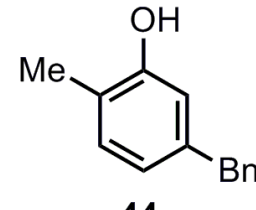

F2 - Acquisition Parameter Date_ 20190326 Time

INSTRUM

44

PUIPROG

z151340 spect

Zgog30

TD

SOLVENT

NS

DS

FIDRES

$\mathrm{AQ}$

RG

DW

TE

TI

D 1
D 11
TD 0

TD 0

$\mathrm{SF} O 1$

NUC1

$\mathrm{P} 1$

P LW1

$\mathrm{SFO} 2$

$\mathrm{NUC} 2$

CPDPRG [ 2

PCPD 2

PLW2

P LW1 2

65536

$\mathrm{CDCl} 3$

30

$42613.637 \mathrm{~Hz}$

$1.300465 \mathrm{~Hz}$

$0.7689557 \mathrm{sec}$ 203

11.733 usec 18.00 usec $303.2 \mathrm{~K}$

$2.00000000 \mathrm{sec}$ $0.03000000 \mathrm{sec}$

$176.0362620 \mathrm{MHz}$ $13 \mathrm{C}$

11.25 usec

$29.00000000 \mathrm{~W}$

$700.0098000 \mathrm{MHz}$

P LW1 3

waltz 16

65.00 user

$13.00000000 \mathrm{~W}$

$0.27768999 \mathrm{~W}$

$0.13982999 \mathrm{~W}$

F2 - Processing parameter

SI 131072

SF $\quad 176.0169002 \mathrm{MHz}$

WDW

EM

$\mathrm{SSB}$

LB

0

$1.00 \mathrm{~Hz}$

1.40

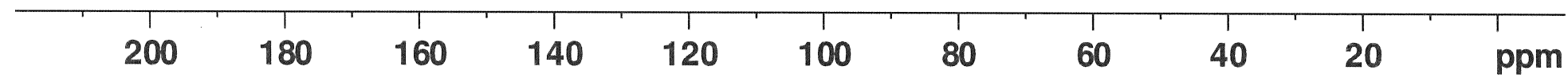


<smiles>Cc1cccc(Br)c1O</smiles>

45

\section{Current Data Parameters NAME \\ EXPNO \\ PROCNO \\ $\mathrm{ZX}-2-152$}

F2 - Acquisition Parameter

Date_ 20180528

Time 16.27

INSTRUM spect

PROBHD $5 \mathrm{~mm}$ PABBO BB-

PULPROG Z

TD 65536

SOLVENT CDCl3

DS

SWH

FIDRES

AQ

RG

DW

$\mathrm{DE}$

TDO

32

$8278.146 \mathrm{~Hz}$

$0.126314 \mathrm{~Hz}$ $3.9583745 \mathrm{sec}$ 90.5

60.400 usec 6.50 usec

$1.00000000 \mathrm{sec}$ $==$ CHANNEL $\mathrm{f}$

NUC

$\mathrm{P} 1$

PL1

PLIW

SFO1
$\mathrm{dB}$
$0.27361584 \mathrm{~W}$

14.00 usec

2 - Processing parameters

SE

(

SSB

LB

$\mathrm{PC}$

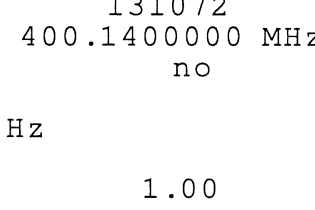

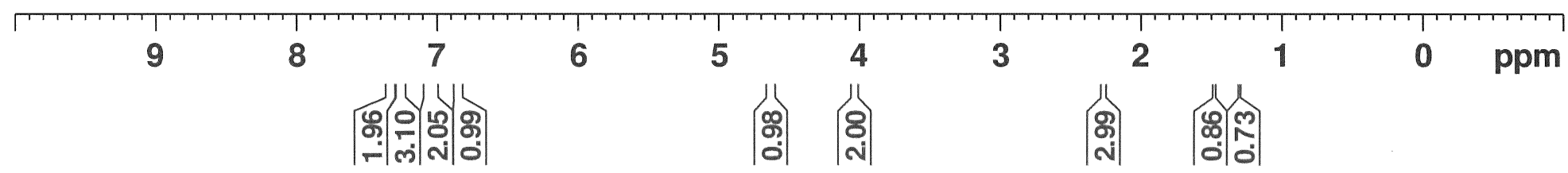


N

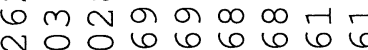
- $\cdot$. r -106666
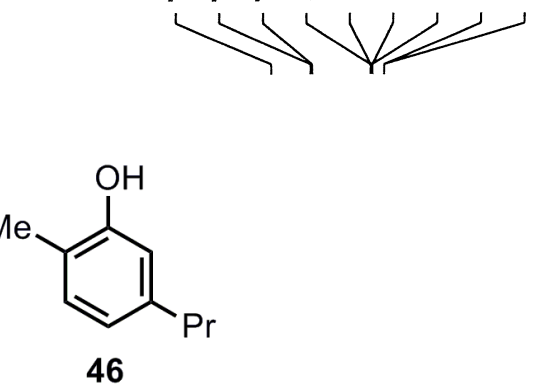

の

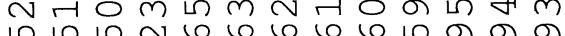

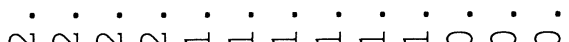

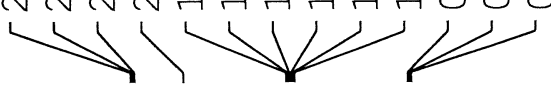

Current Data Parameters NAME

EXPNO

PROCNO

F2 - Acquisition Parameters

Date__ 20190422

Time

20190422

INSTRUM

PROBHD

PULPROG

2151340_0001 (

SOLVENT

DS

SWH

FIDRES

AQ

RG

DW

$\mathrm{DE}$

TD 1

$\mathrm{SFO} 1$

NUC1

P1

PLW1

zg30

$\mathrm{CDCl} 3$ 16

$10504.202 \mathrm{~Hz}$

$0.320563 \mathrm{~Hz}$

$3.1195135 \mathrm{sec}$ 5.6

47.600 usec 10.00 usec $303.2 \mathrm{~K}$

$1.00000000 \mathrm{sec}$

1.00000000

$1 \mathrm{H}$ $1 \mathrm{H}$
50 use

$13.00000000 \mathrm{~W}$

F2 - Processing parameters SI 131072

SF $\quad 700.0070147 \mathrm{MHz}$

WDW

SSB

SSB

$\mathrm{LB}$
$\mathrm{GB}$

GB
PC

0

$0 \mathrm{~Hz}$

$$
\text { no }
$$

1.00

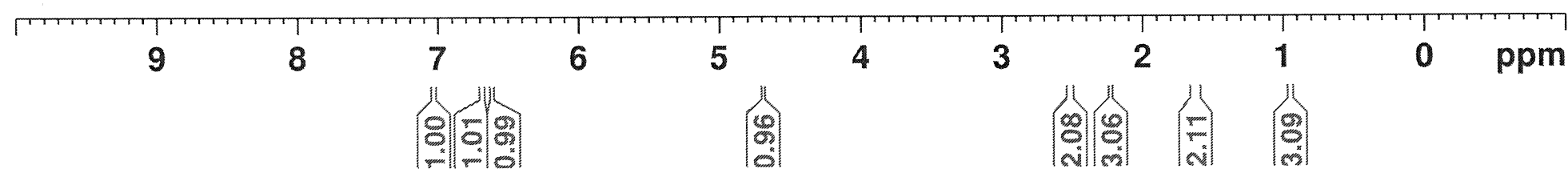




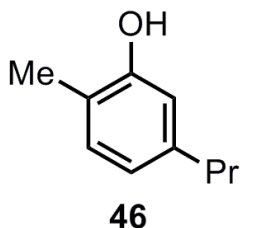
Current Data Parameters

F2 - Acquisition Parameter

Date_ 20190422

Time

$15.51 \mathrm{~h}$

INSTRUM

PROBHD

PULPROG spect

TD Z151340_0001

SOLVENT 65536

NS

SWH

FIDRES

$\mathrm{AQ}$

RG

DW

$\mathrm{DW}$
$\mathrm{TE}$

D 1

D 11

TD 0

SFO1

$\mathrm{NUC1}$

P 1

P LW1

$\mathrm{SFO} 2$

NUC2

CPDPRG [ 2

PCPD 2

P LW2

PLW1 2

PLW1 3

29

$42613.637 \mathrm{~Hz}$

1. $300465 \mathrm{~Hz}$

$0.7689557 \mathrm{sec}$ 203

11.733 usec 18.00 usec $303.2 \mathrm{~K}$

$2.00000000 \mathrm{sec}$ $0.03000000 \mathrm{sec}$ $176.0362620 \mathrm{MHz}$ $13 \mathrm{C}$

11.25 usec

$29.00000000 \mathrm{~W}$ $29.00000000 \mathrm{~W}$
$700.0098000 \mathrm{MH}$

$$
\begin{gathered}
1 \mathrm{H} \\
\text { waltz } 16
\end{gathered}
$$

65.00 use

$13.00000000 \mathrm{~W}$

$0.27768999 \mathrm{~W}$

$0.13982999 \mathrm{~W}$

F2 - Processing parameter

SI 131072

SF $\quad 176.0169002 \mathrm{MHz}$

WDW

$176.0169002 \mathrm{MHz}$
EM

SSB $\quad 0$

$L B$

$\mathrm{GB}$

$1.00 \mathrm{~Hz}$

1.40

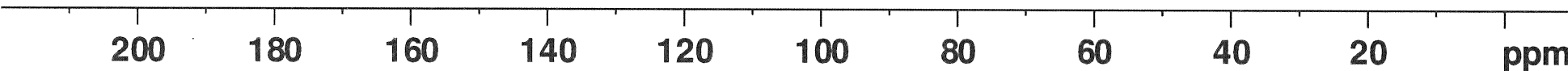




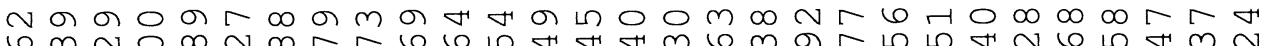

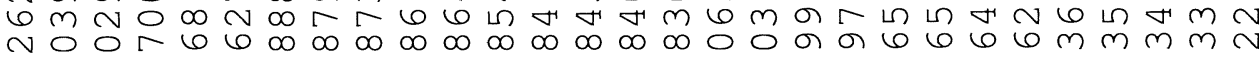

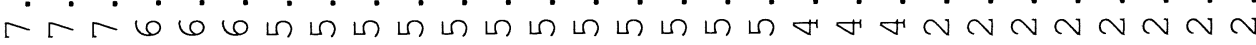

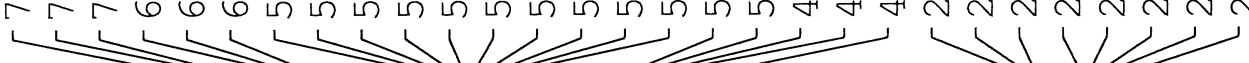

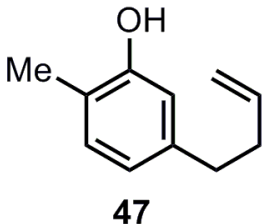

Current Data Parameters

NAME

EXPNO

PROCNO

$\mathrm{XZ}-4-37$

F2 - Acquisition Parameters

Date_ 20191013

Time

181013

INSTRUM

PROBHD

18.13

PULPROG

2151340_0001

TD

SOLVENT

NS

DS

SWH

FIDRES

AQ

RG

DW

$\mathrm{DE}$

(1)

TD 0

$\mathrm{SFO}$

NUC1

P 1

PLW1

zg30
65536

CDCl3

16

F2 - Processing parameters

SI 131072

SF $\quad 700.0070113 \mathrm{MHz}$

WDW

LB

$10504.202 \mathrm{~Hz}$

$0.320563 \mathrm{~Hz}$

$3.1195135 \mathrm{sec}$

47.600 usec

20.00 usec

$303.3 \mathrm{~K}$

$1.00000000 \mathrm{sec}$

$700.0115500 \mathrm{MHz}$

3.17 usec

9.50 usec

$13.00000000 \mathrm{~W}$
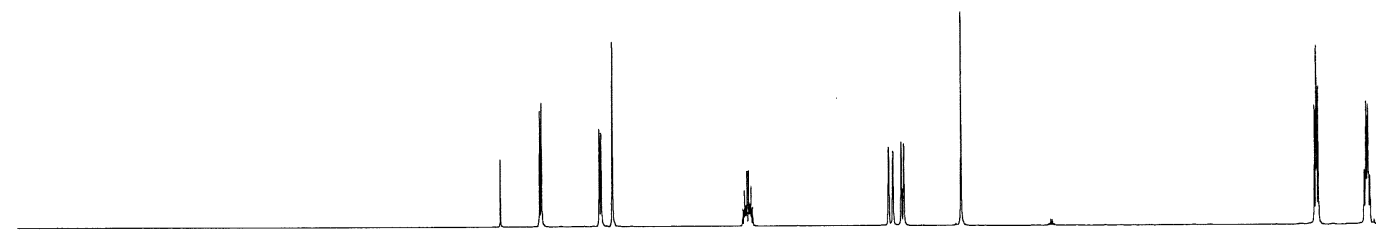

700.0070113

EM
0
0

$0.30 \mathrm{~Hz}$

1.00

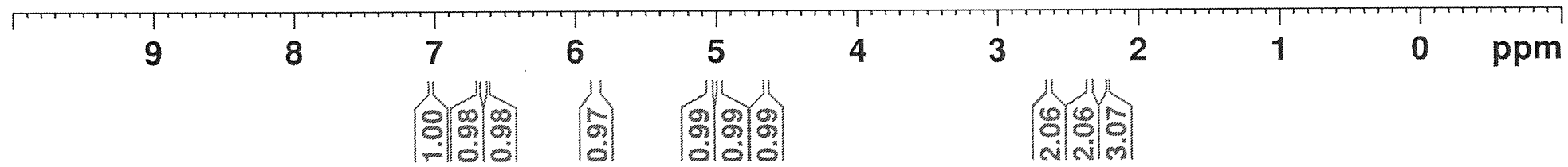




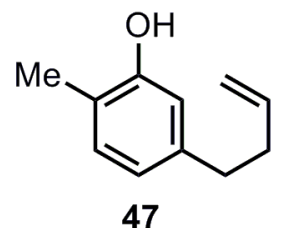

Current Data Parameters

F2 - Acquisition Parameters

Date__ 20191013

Time

INSTRUM

PROBHD

PULPROG

spect
$2151340 \quad 0001$

$$
\text { TD }
$$

SOLVENT

NS

DS

SWH

FIDRES

AQ

DW

DE

TE

DI

TD 0

TDO

NUC1

$\mathrm{P} 0$

P 1

PLW1

$\mathrm{SFO} 2$

CPDPRG [2

PCPD 2

(I) 2

PLW12
PLW13

zgpg30
65536

$\mathrm{CDCl} 3$

4
42613.637
$\mathrm{~Hz}$

$1.300465 \mathrm{~Hz}$

$0.7689557 \mathrm{sec}$

$$
203
$$

11.733 usec

18.00 usec

$$
\begin{aligned}
& 18.00 \text { us } \\
& 303.2 \mathrm{~K}
\end{aligned}
$$

$2.00000000 \mathrm{sec}$

$0.03000000 \mathrm{sec}$

$176.0362620 \mathrm{MHz}$ $13 \mathrm{C}$

3.75 usec
1.25 usec

$29.00000000 \mathrm{~W}$

$700.0098000 \mathrm{MHz}$

F2 - Processing parameters

SI 131072

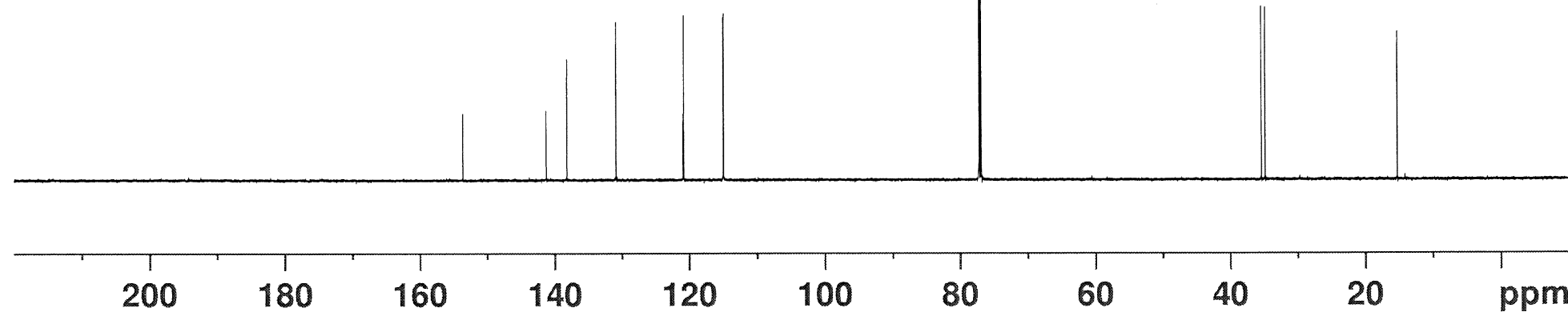

SSB

$176.0169002 \mathrm{MHz}$

$$
\begin{array}{r}
1.00 \mathrm{~Hz} \\
0 \\
1.40
\end{array}
$$




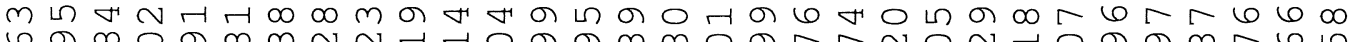

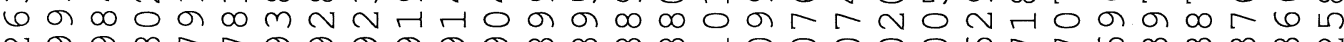
$\sim 0 \% \mathrm{~mm} \sim$ -

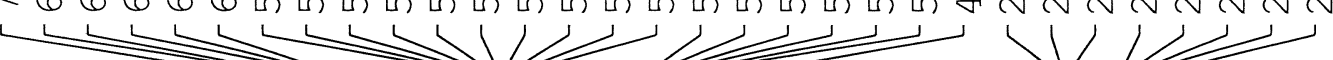

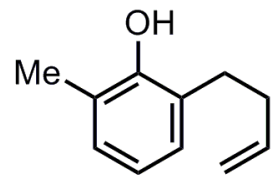

48
Current Data Parameters

$$
\text { NAME }
$$

EXPNO

PROCNO

$\mathrm{XZ}-4-46$

Acquisition Parameters

Time

INSTRUM

PROBHD

PROBHD

TD

SOLVENT

NS

DS

FIDRES

$\mathrm{AQ}$

RG

DW

$\mathrm{DE}$

TE

TI

TDO

$\mathrm{SFO1}$

$\mathrm{PO}$

$\mathrm{P} 0$
$\mathrm{P} 1$

PLW1

SI 131072

SF $\quad 700.0070149 \mathrm{MHz}$

WDW

$\mathrm{SSB}$

$\mathrm{LB}$

GB
PC

EM
0
$0.30 \mathrm{~Hz}$
0
1.00
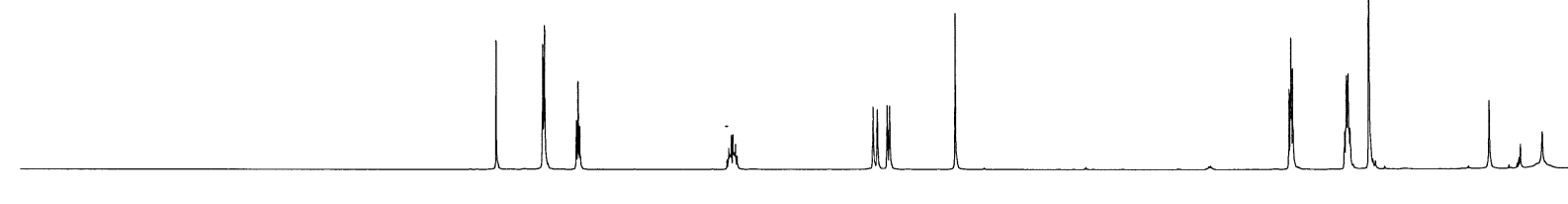

3

2

1

60

क्य

in 


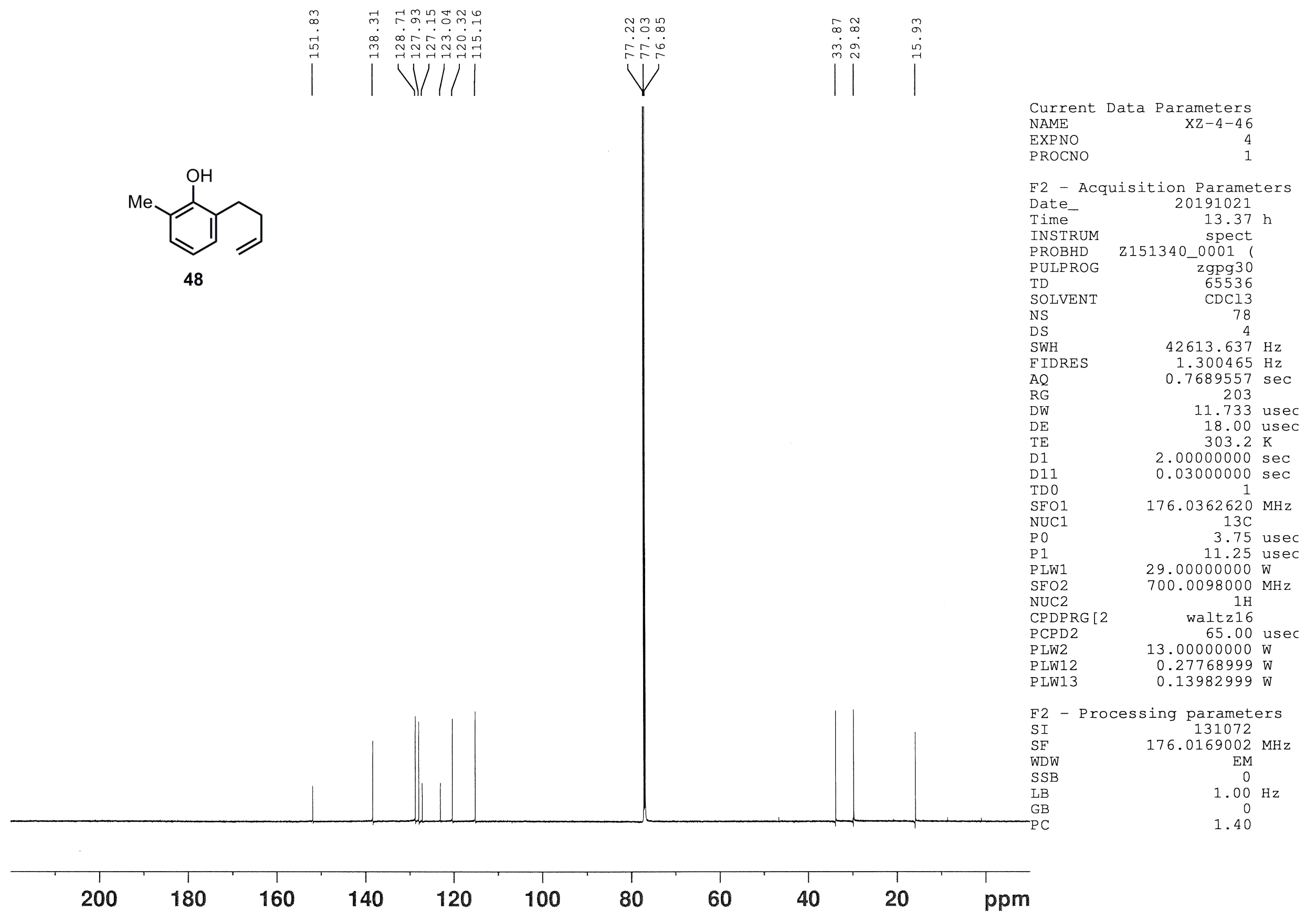




\begin{tabular}{|c|c|c|c|}
\hline & & & \\
\hline 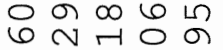 & 6 & M & $\begin{array}{ll}0 & \infty \\
0 & \infty\end{array}$ \\
\hline त वंर & 6 & $6666 \mathrm{~N} N$ & $N$ H \\
\hline ن & $\dot{\gamma}$ & $\dot{\sim} \dot{\sim} \dot{\sim} \dot{\sim} \dot{\sim}$ & $\dot{H}$ \\
\hline & & & \\
\hline
\end{tabular}

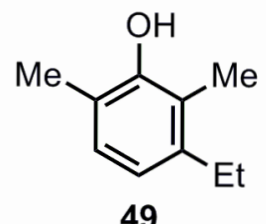

Current Data Parameters

F2 - Acquisition Parameter Date__ 20181119 Time $\quad 16.01 \mathrm{~h}$ INSTRUM spect PROBHD Z151340_0001 PULPROG zg30 TD 65536 SOLVENT CDC13 $\begin{array}{lc}\text { SOLVENT } & \text { CDCl3 } \\ \text { NS } & 16\end{array}$ NS

$10504.202 \mathrm{~Hz}$ FIDRES $\quad 0.320563 \mathrm{~Hz}$ $\mathrm{AQ} \quad 3.1195135 \mathrm{sec}$ RG

$1.00000000 \mathrm{sec}$ $700.0115500 \mathrm{MHz}$ P LW1 9. 50 usec $13.00000000 \mathrm{~W}$

F2 - Processing parameters SI 131072 SF $\quad 700.0070176 \mathrm{MHz}$ WDW

SSB

$\mathrm{LB}$
$\mathrm{GB}$ 0 $\mathrm{Hz}$

$$
\text { no }
$$

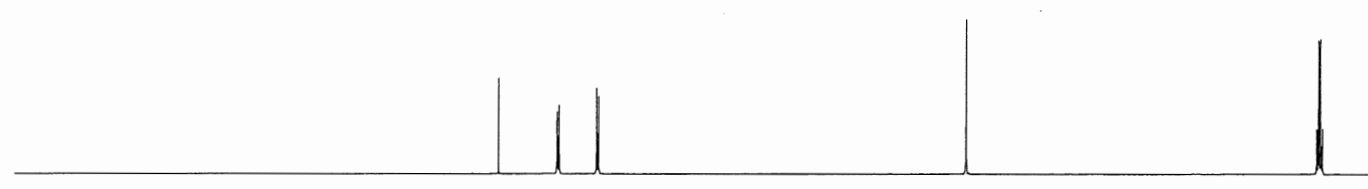

$P C$

1.00

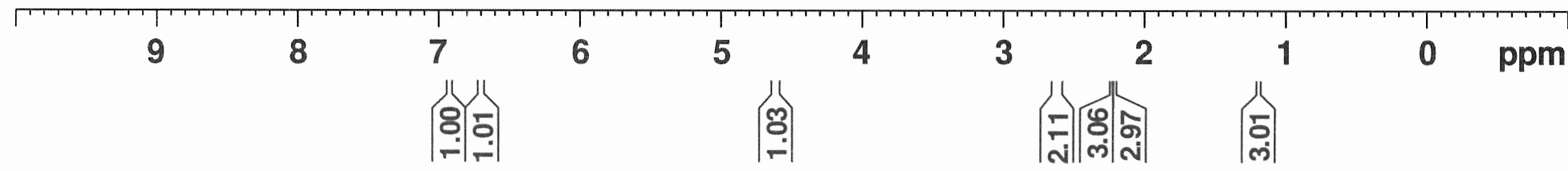



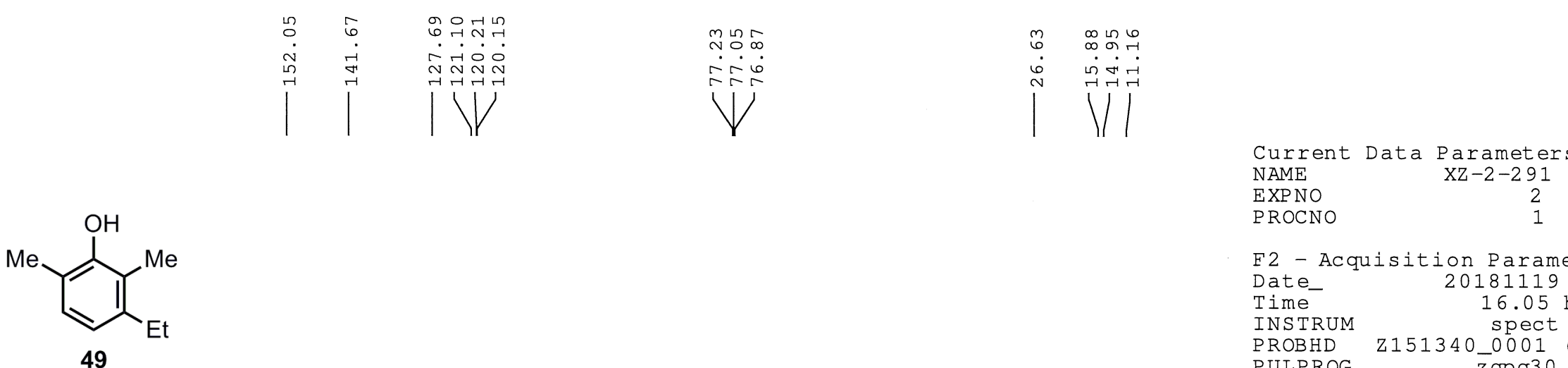

PROCNO

F2 - Acquisition Parameter

Date__ 20181119

Time

$16.05 \mathrm{~h}$

INSTRUM

Z151340_0001(

49

PULPROG

SOLVENT

NS

SWH

FIDRES

AQ

RG

DW

TE

D 1

D 11

TEO

SFO1

Ne1

1

P LW1

$\mathrm{SEO} 2$
NUC2

NUC2

CPDPRG [ 2

PCP D 2

LLW2

PLW12

P LW1 3

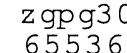

6536

$\mathrm{CDCl} 3$

34

$42613.637 \mathrm{~Hz}$

$1.300465 \mathrm{~Hz}$

$0.7689557 \mathrm{sec}$

1.733 usec 18.00 usec $303.2 \mathrm{~K}$

$2.00000000 \mathrm{sec}$ $0.03000000 \mathrm{sec}$

$176.0362620 \mathrm{MHz}$

11.25 usec

$29.00000000 \mathrm{~W}$

$700.0098000 \mathrm{MHz}$

$$
\begin{gathered}
1 \mathrm{H} \\
\text { waltz } 16
\end{gathered}
$$

65.00 use

$13.00000000 \mathrm{~W}$

$0.27768999 \mathrm{~W}$

$0.13982999 \mathrm{~W}$

F2 - Processing parameters

SI 131072

SF $\quad 176.0169002 \mathrm{MHz}$

WDW EM

SSB $\quad 0$

$L B$

PB

$1.00 \mathrm{~Hz}$

1.40 


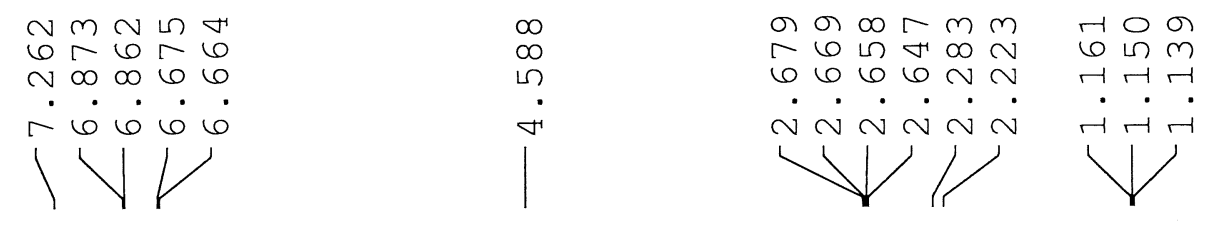

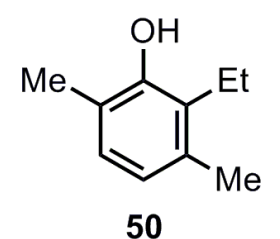

Current Data Parameters

F2 - Acquisition Parameters Date__ 20190919

Time $10.03 \mathrm{~h}$

INSTRUM

PROBHD

PUIPROG

z151340 0001

SOLVENT

NS

DS

SWH

FIDRES

AQ

RG

DW

$\mathrm{DE}$

TE

D1

TD 0

$\mathrm{SFO}$

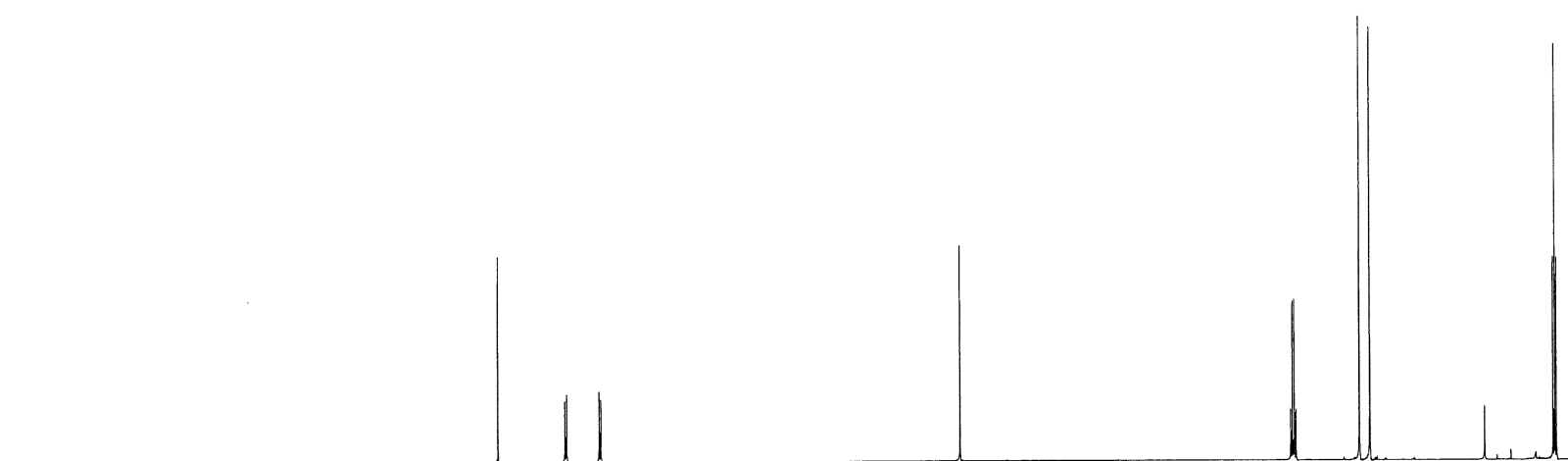

NUC1

$\mathrm{PO}$

$\mathrm{P} 1$

PLW1

$\mathrm{zg} 30$
65536

CDC13

$10504.202 \mathrm{~Hz}$

$0.320563 \mathrm{~Hz}$

$0.1195135 \mathrm{sec}$
32

47.600 usec

20.00 usec

$303.2 \mathrm{~K}$

$1.00000000 \mathrm{sec}$

1

$700.0115500 \mathrm{MHz}$

$1 \mathrm{H}$
$3.17 \mathrm{use}$

3.17 usec
9.50 usec

F2 - Processing parameters

SI 131072

SF $\quad 700.0070168 \mathrm{MHz}$

WDW

SSB

LB

LB

PC

700.0070168
no

no
0
0
0
$0 z z$
1.0

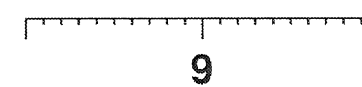

8

7

6

5

4

wa

3

2

(1)

1

(1)

\section{ppm}




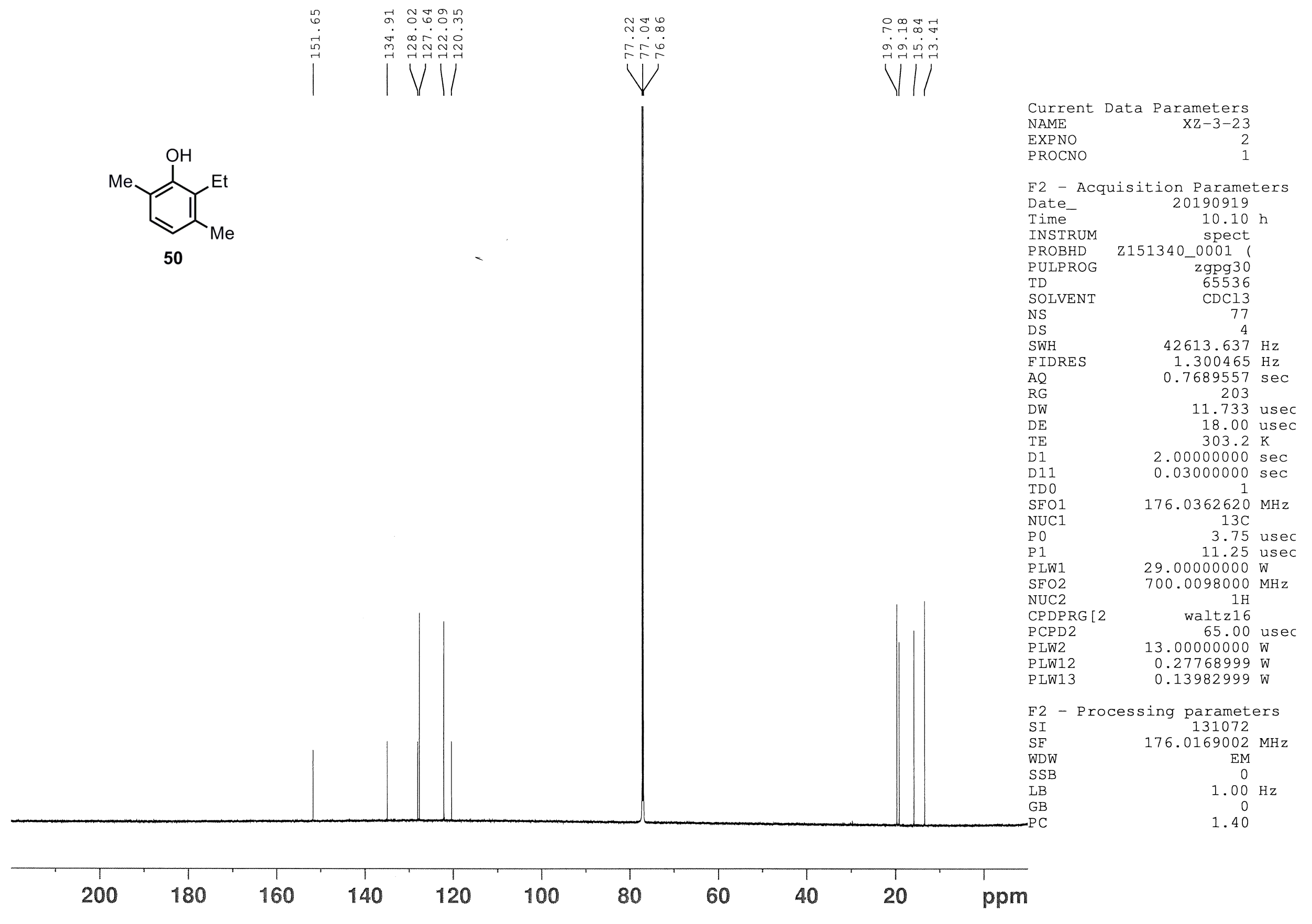


<smiles>Cc1ccc(-c2ccccc2)c([N+](=O)[O-])c1O</smiles>

51 mo

$m \rightarrow$

$\dot{\sim} \dot{0}$

11
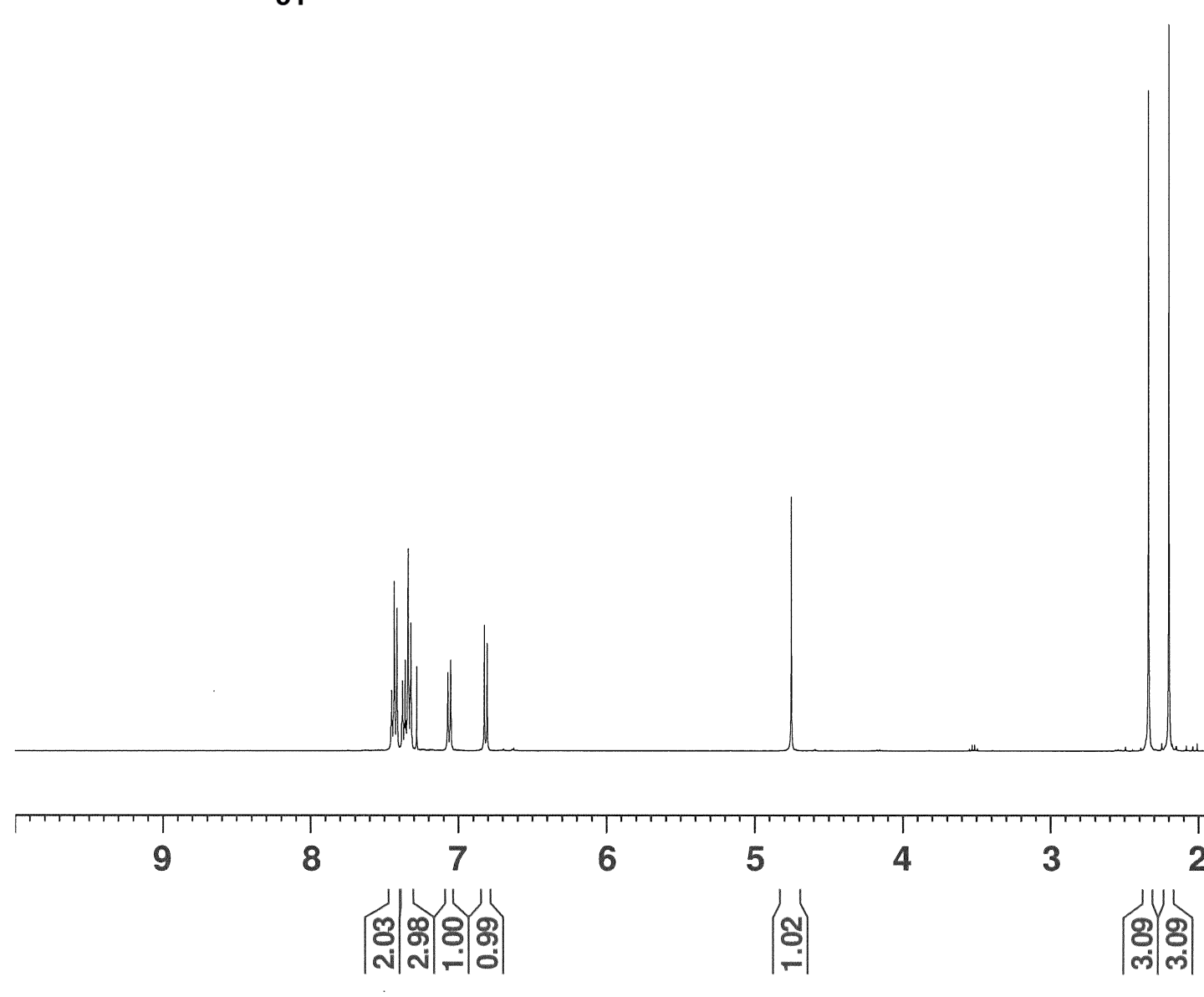

3

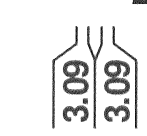
Current Data Parameters $\mathrm{zX}-2-121$
EXPNO
PROCNO

1

F2 - Acquisition Parameter

$\begin{array}{lc}\text { Date_ } & 20180430 \\ \text { Time } & 20.31\end{array}$

20.31

$5 \mathrm{~mm}$ PABBO BB-

SOLVENT

NO

DS

DS

FIDRES

AQ

RG

$\mathrm{DE}$

TE

T 1

$\mathrm{Zg} 30$
65536

CDCl3

32

$8278.146 \mathrm{~Hz}$

$0.126314 \mathrm{~Hz}$ $3.9583745 \mathrm{sec}$

128

60.400 usec 6.50 usec $303.4 \mathrm{~K}$

$1.00000000 \mathrm{sec}$

NUC 1

$\mathrm{P} 1$

PL1

PLIW

$\mathrm{SFO1}$
14.00 usec
$0 \mathrm{~dB}$
$10.27361584 \mathrm{~W}$

F2 - Processing parameters SI 131072 
ชิ

$\sim 00$ ด न $\infty \infty \infty \infty$

- r $\dot{0} \dot{0} \dot{0} \dot{0}$

r.

$\rightarrow$

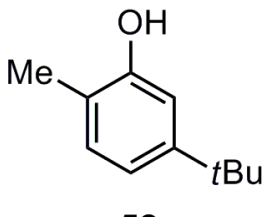

52

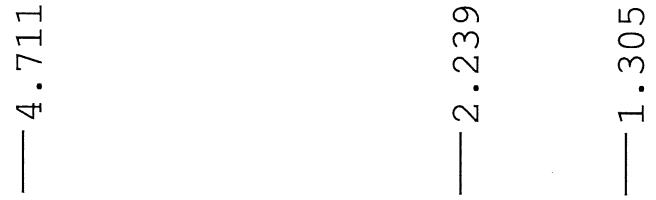

Current Data Parameter NAME

EXPNO $\mathrm{XZ}-3-106$ PROCNO

F2 - Acquisition Parameter Date__ 20190326 Time $\quad 12.59 \mathrm{~h}$

INSTRUM spect

PROBHD Z151340_0001

PULPROG zg30

SOLVENT

NS

FIDRES

$A Q$

RG

DW

TE

D 1

TD 0

$\mathrm{SFO} 1$

P 1

P LW1

$$
65536
$$

$\mathrm{CDCl} 3$

16

$10504.202 \mathrm{~Hz}$

$0.320563 \mathrm{~Hz}$

$3.1195135 \mathrm{sec}$ 5.6

47.600 usec

10.00 usec

$303.2 \mathrm{~K}$

$1.00000000 \mathrm{sec}$

1

$700.0115500 \mathrm{MHz}$

9.50 usec

$13.00000000 \mathrm{~W}$

F2 - Processing parameters

WDW

$700.0070157 \mathrm{MHz}$

$\mathrm{SSB}$

LB

GB

0

$0 \mathrm{~Hz}$

0

1.00

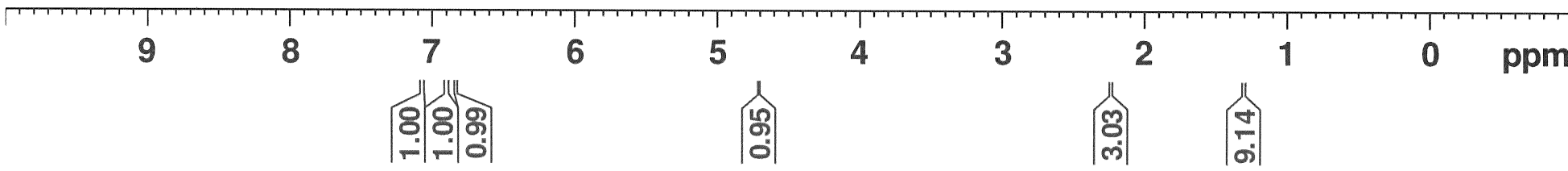




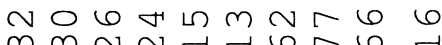
$66 \pi$ 은

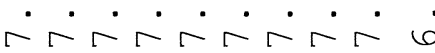

3

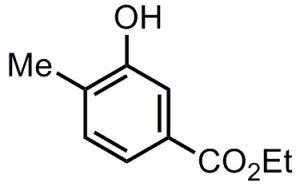

53

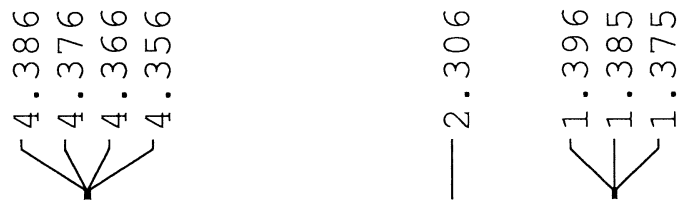

Current Data Parameters NAME

EXPNO

PROCNO

F2 - Acquisition Parameters

Date_ 20190819

Time

$17.20 \mathrm{~h}$

INSTRUM

PROBHD

PROBHD

TD

SOLVENT

NS

DS

SWH

FIDRES

AQ
RG
DW

DW

$\mathrm{DE}$

$\mathrm{TE}$

D 1

$\mathrm{SFO} 1$

NUC

P 1

PLW1

spect

0001

zg30

CDC13

16

2
$10504.202 \mathrm{~Hz}$

$0.320563 \mathrm{~Hz}$

$3.1195135 \mathrm{sec}$ 25.4

47.600 usec

20.00 usec

$303.3 \mathrm{~K}$

$1.00000000 \mathrm{sec}$

$700.0115500^{1} \mathrm{MHz}$ $1 \mathrm{H}$

9.50 usec

$13.00000000 \mathrm{~W}$

F2 - Processing parameters SI 131072

SF $\quad 700.0070151 \mathrm{MHz}$

WDW

WDW

SSB

LB

PC

0

$\mathrm{Hz}$

no

1.00

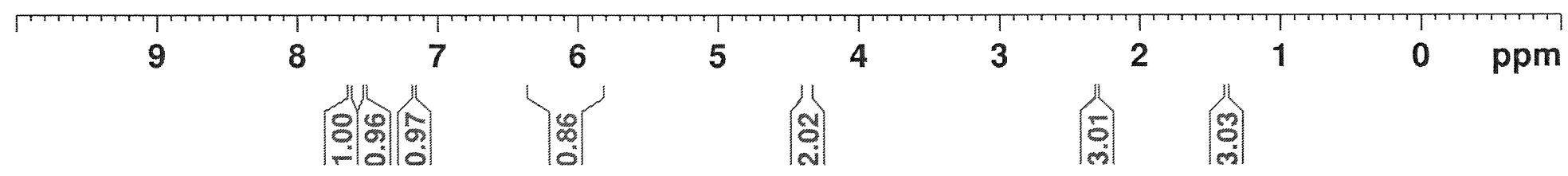




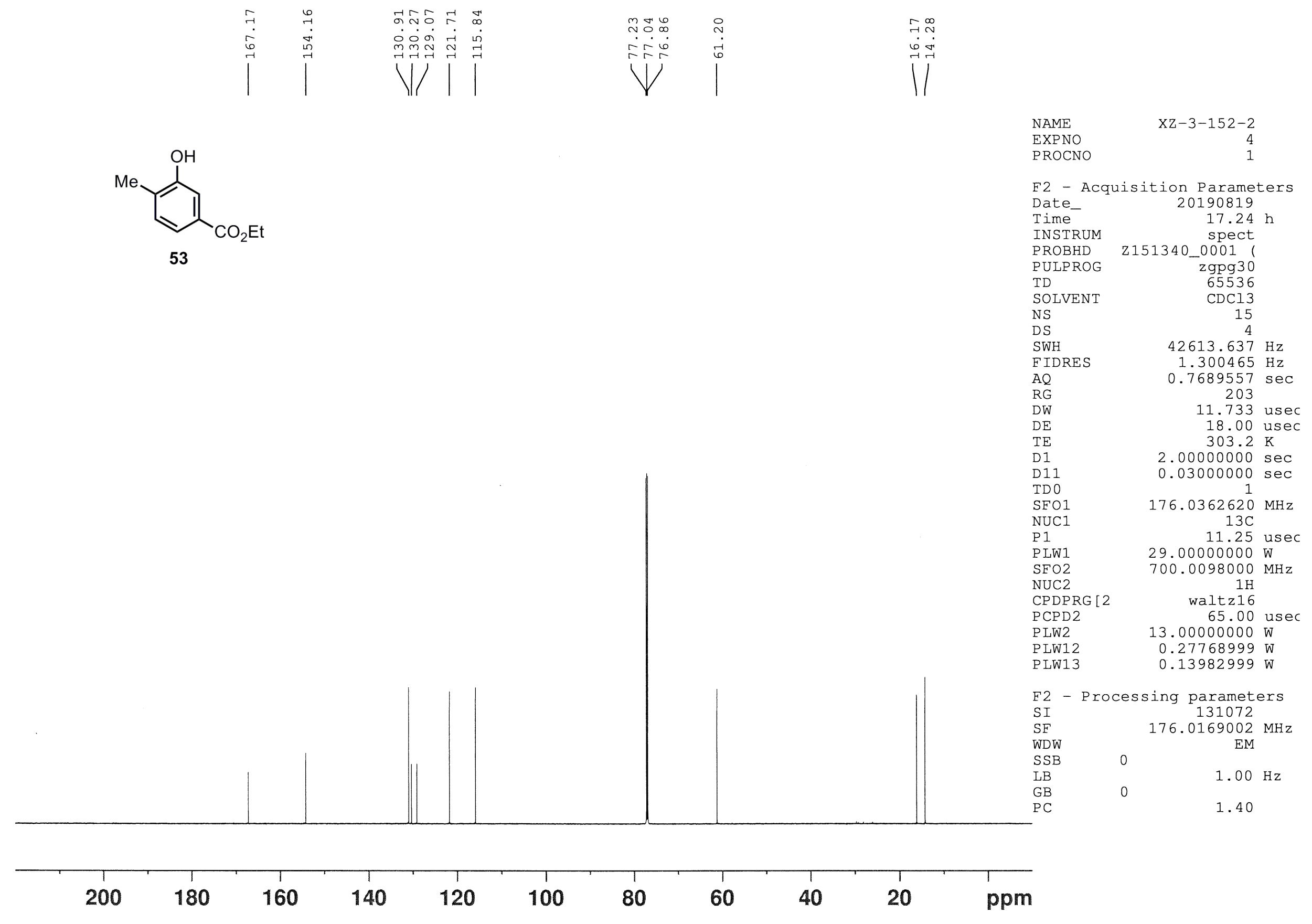


0006 in

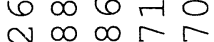

$\cdot \cdot \cdot \cdot \cdot$

- 666

11<smiles>CCc1ccc(CC(C)C)c(O)c1C</smiles>

54

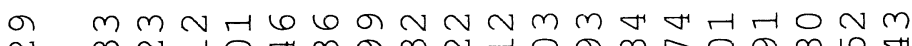

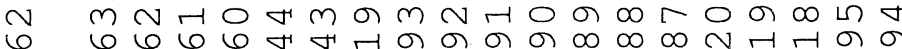

$\dot{\sim} \dot{\sim} \dot{\sim} \dot{\sim} \dot{\sim} \dot{\sim} \dot{\sim} \dot{\sim} \dot{\sim} \dot{\sim} \dot{\sim} \dot{\sim} \dot{\sim} \dot{0}$

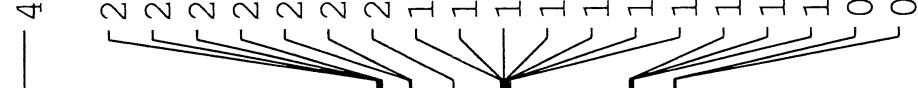

Current Data Parameters

NAME

$\mathrm{Xz}-3-3$

EXPNO

PROCNO

F2 - Acquisition Parameters

Date_ 20181210

Time $13.40 \mathrm{~h}$

INSTRUM spect

PROBHD Z151340_0001

PULPROG zg30

TD 65536

SOLVENT CDCl3

NS

DS

SWH $\quad 10504.202 \mathrm{~Hz}$

FIDRES $\quad 0.320563 \mathrm{~Hz}$

$\mathrm{AQ} \quad 3.1195135 \mathrm{sec}$

RG $\quad 5.6$

DW

$\mathrm{DE}$

TE

D1

TFO 1

NUC1

P1

$\mathrm{P} 1$
$\mathrm{PLW} 1$

47.600 usec

10.00 usec

$298.2 \mathrm{~K}$

$1.00000000 \mathrm{sec}$

$700.0115500^{1} \mathrm{MHz}$

$$
1
$$

$1 \mathrm{H}$

$13.00000000 \mathrm{~W}$

$\begin{array}{lcc}\text { F2 } & \text { Processing parameters } \\ \text { SI } & 131072 \\ \text { SF } & 700.0070166 \mathrm{MHz} \\ \text { WDW } & 0 & \mathrm{no} \\ \text { SSB } & 0 & \\ \text { LB } & 0 \mathrm{~Hz} & \\ \text { GB } & 0 & 1.00\end{array}$

9

\begin{abstract}
8
\end{abstract}
7
015
0
6

5 $\int_{0}^{1}$

43

3

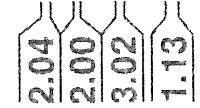

2 1
N)
0
$m$
$m$
0 


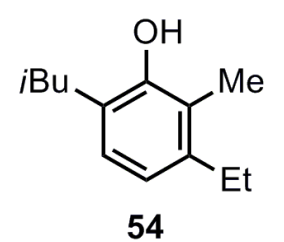

54
Current Data Parameters

F2 - Acquisition Parameter Date_ 20181210 Time

INSTRUM

PROBHD

PULPROG

$13.49 \mathrm{~h}$

TD

SOLVENT

NS

DS

SWH

FIDRES

AQ

RG

DW

DE

D 1

D 1
D 11
TD 0

$\mathrm{SFO1}$

$\mathrm{P} 1$

P LW1

$\mathrm{SFO} 2$

NUC2

CPDPRG [ 2

PCP D 2

P LW2

P LW1 2

Z151340_0001 (

65536

CDCl3

50

$42613.637 \mathrm{~Hz}$

$1.300465 \mathrm{~Hz}$ $0.7689557 \mathrm{sec}$ 203

11.733 usec 18.00 usec $298.2 \mathrm{~K}$

$2.00000000 \mathrm{sec}$ $0.03000000 \mathrm{sec}$ 1 $\mathrm{MHz}$ $13 \mathrm{C}$

11.25 usec 29.00000000 $700.0098000 \mathrm{MHz}$

$$
\begin{gathered}
1 \mathrm{H} \\
\text { waltz } 16 \\
65.00
\end{gathered}
$$

3.00000000 us

$0.27768999 \mathrm{~W}$

$0.13982999 \mathrm{~W}$

F2 - Processing parameters $\begin{array}{lc}\text { SI } & 131072 \\ \text { SF } & 176.0169002 \mathrm{MHz}\end{array}$ WDW $\mathrm{SSB}$ LB

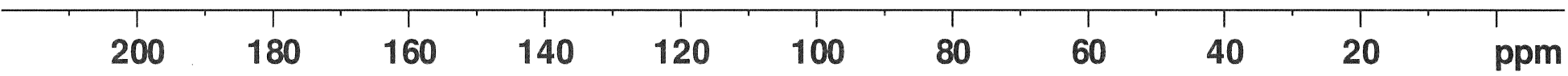




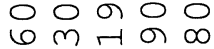

$\sim \infty \infty 66$

$\therefore 6$ ن
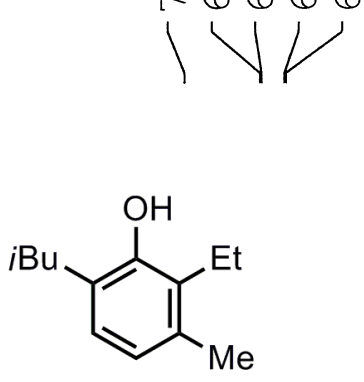

55

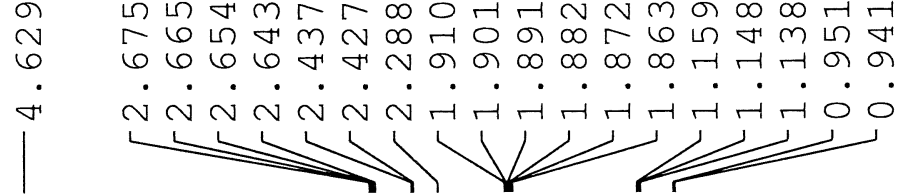

Current Data Parameters

NAME

EXPNO

PROCNO

$\mathrm{XZ}-3-32$

F2 - Acquisition Parameters

Date__ 20181210

Time $\quad 20.09 \mathrm{~h}$

INSTRUM spect

PROBHD Z151340_0001 (

PULPROG zg30

TD 65536

SOLVENT CDCl3

NS

DS

SWH

FIDRES

AQ

RG

DW

DE

TE

D1 0

$\mathrm{SFO} 1$

NUC1

$\mathrm{P} 1$

PLW1

16
2
$10504.202 \mathrm{~Hz}$

$0.320563 \mathrm{~Hz}$

$3.1195135 \mathrm{sec}$

47.600 use

$298.2 \mathrm{~K}$

$1.00000000 \mathrm{sec}$

700.0115500

9.50 usec

$13.00000000 \mathrm{~W}$

F2 - Processing parameters

SI 131072

SF $\quad 700.0070183 \mathrm{MHz}$

WDW

SSB

LB
GB

PC

$\mathrm{O} \mathrm{Hz}$

$$
\text { no }
$$

1.00

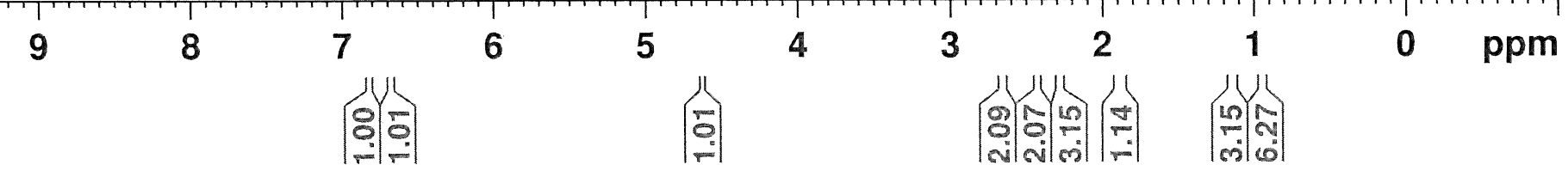




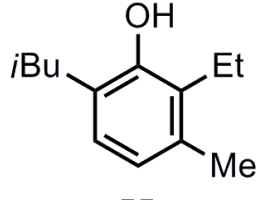

55

$\begin{array}{lc}\text { Current } & \text { Data } \\ \text { NAME } & \text { XZ }-3-32 \\ \text { EXPNO } & 2 \\ \text { PROCNO } & 1\end{array}$

F2 - Acquisition Parameter

Time

INSTRUM

PROBHD

PULPROG

20181210
20.15

TD

SOLVENT

NS

DS

FIDRES

$\mathrm{AQ}$

RG

$D W$

DE

1

D1

TD 0

$\mathrm{SFO1}$

NUC1

P 1

P LW1

$\mathrm{SFO} 2$

$\mathrm{NUC} 2$

CPDPRG [ 2

PCPD 2

P LW2

P LW1 2

PLW1 3

Z151340_0001

z gpg30

$\mathrm{CDC} 13$
62
4

$42613.637 \mathrm{~Hz}$

$1.300465 \mathrm{~Hz}$

$0.7689557 \mathrm{sec}$ 203

11.733 usec 18.00 usec $298.2 \mathrm{~K}$

$2.00000000 \mathrm{sec}$ 0.03000000 $176.0362620 \mathrm{MHz}$ $13 \mathrm{C}$

11.25 usec $29.00000000 \mathrm{~W}$ $700.0098000 \mathrm{MHz}$

$$
\begin{gathered}
1 \mathrm{H} \\
\text { waltz } 16
\end{gathered}
$$

65.00 use

$13.00000000 \mathrm{~W}$

$0.27768999 \mathrm{~W}$

$0.13982999 \mathrm{~W}$

F2 - Processing parameters SI 131072

$\begin{array}{lc}\text { SI } & 131072 \\ \text { SF } & 176.0169002 \mathrm{MHz}\end{array}$

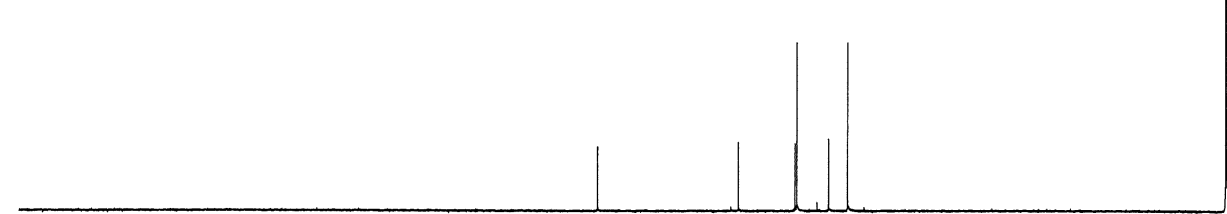

SSB $\quad 0$

LB $\quad 0 \mathrm{~Hz}$

GB

0

1.40

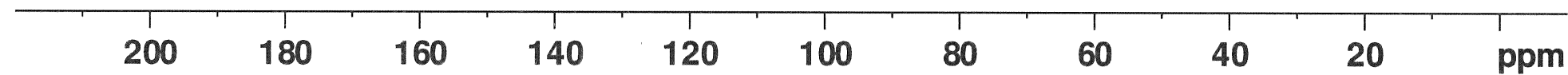


$\infty \sim \sim \infty 6$

6 เ 6 の $\infty$

m.

- -166

$1 / 1$

56

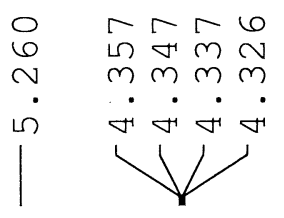

ด

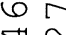

$\sim \sim$

$i$

겅ㅇㅇ잉

मन म

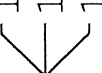

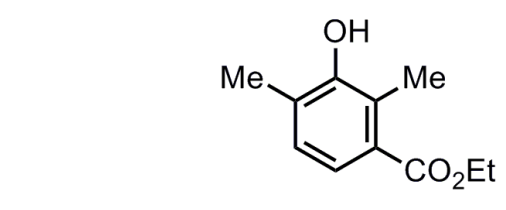

Current Data Parameters NAME

EXPNO

PROCNO

F2 - Acquisition Parameters

Date_ 20190526

$\begin{array}{lr}\text { Time- } & 18.06 \mathrm{~h}\end{array}$

Z151340 0001

PULPROG Zg30

Zg30
PULPROG

SOLVENT

NS

SWH

FIDRES

AQ

RG

DW

DE

TE

1 1

TD 0

$\mathrm{SFO} 1$

NUC1

P 1

PLW1

F2 - Processing parameters
SI

SF

WDW

SDW

LB

GB

65536
$\mathrm{CDC} 13$ 16
2

$10504.202 \mathrm{~Hz}$

$0.320563 \mathrm{~Hz}$

$3.1195135 \mathrm{sec}$ 47.600

10.00 us 10.00 use

$1.00000000 \mathrm{sec}$

$700.0115500 \mathrm{MHz}$ $1 \mathrm{H}$

9.50 usec

$13.00000000 \mathrm{~W}$

$700.0070163 \mathrm{MHz}$

0

$\mathrm{Hz}$

no

1.00

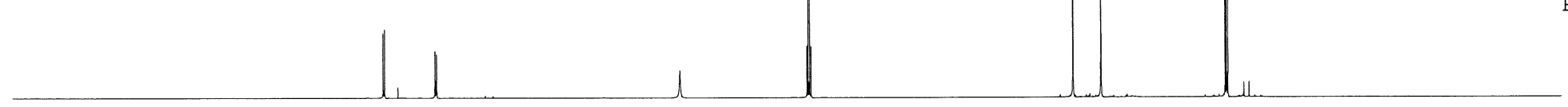

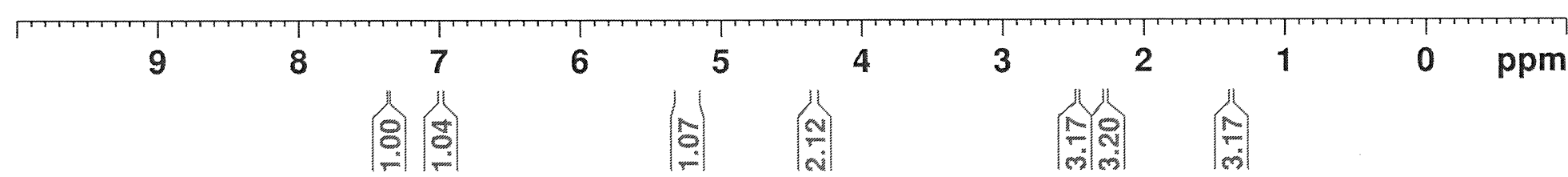




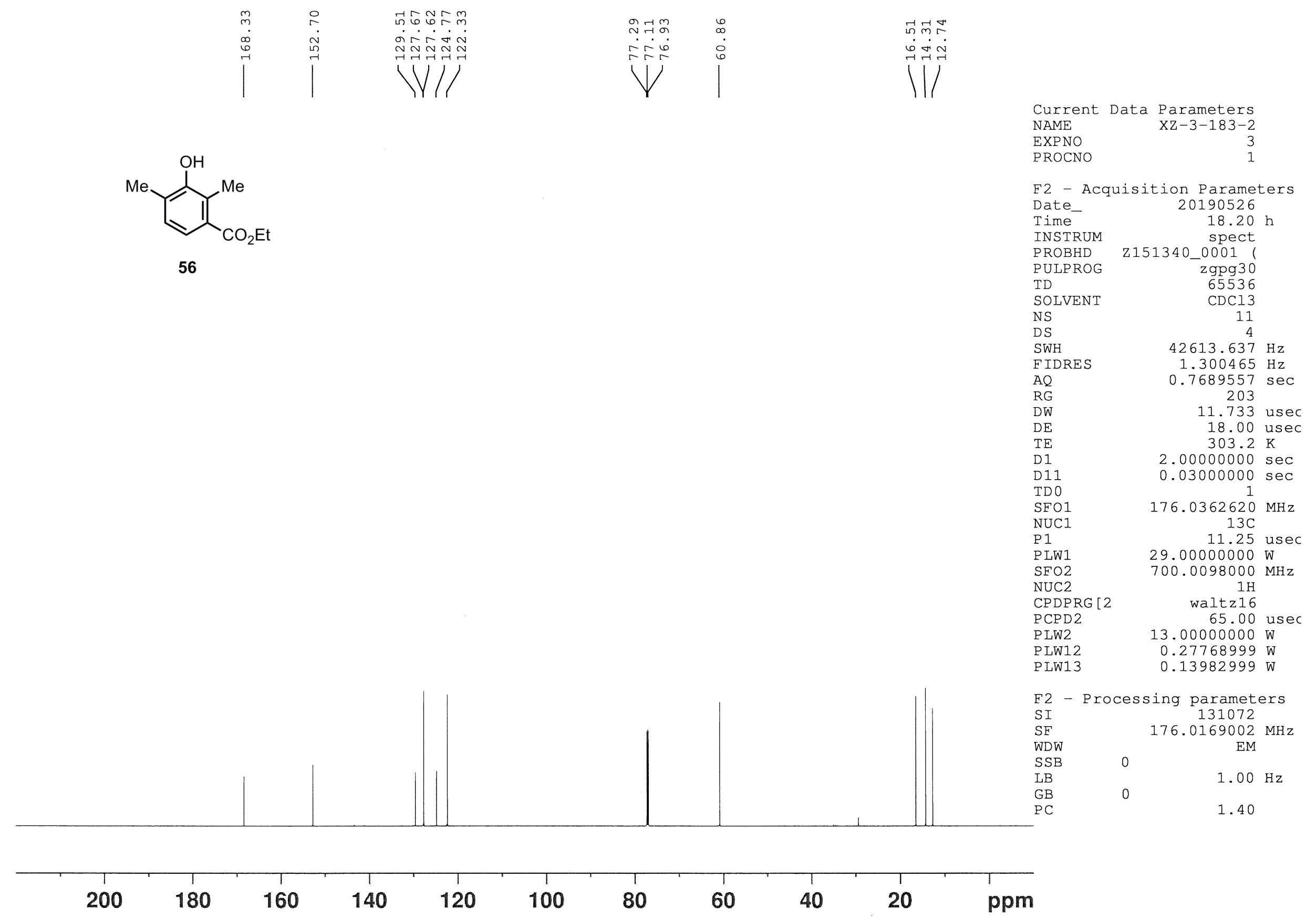



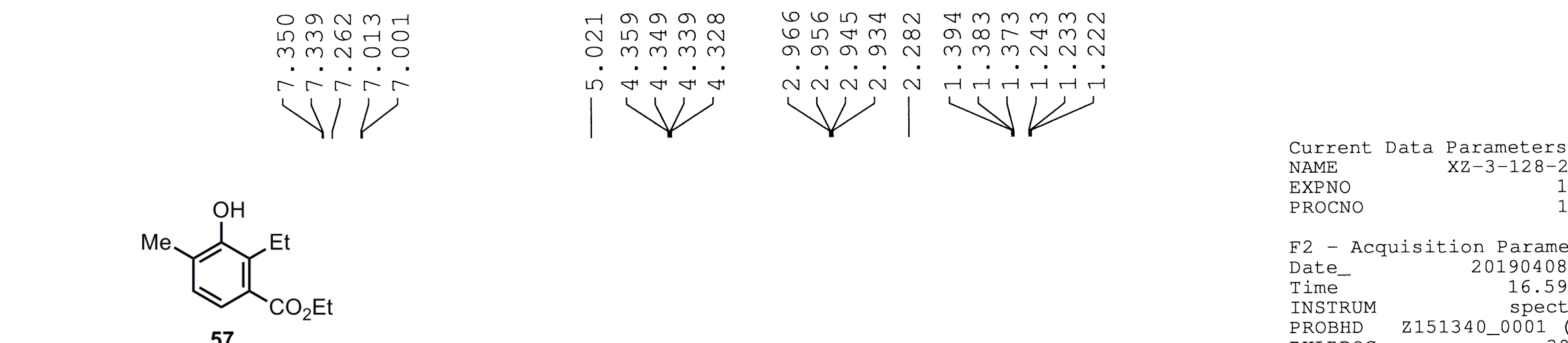

PROCNO

F2 - Acquisition Parameters

Date_ 20190408

Time 16.59

INSTRUM

z151340_0001

SOLVENT

NS

DS

SWH

FIDRES

AQ
RG
DW

DW

$\mathrm{DE}$

$\mathrm{TE}$

TD 0

$\mathrm{SFO} 1$

NUC1

P1

PLW1

spect
$0+00011$
z930
65536

$13.00000000 \mathrm{~W}$

F2 - Processing parameters

SI 131072

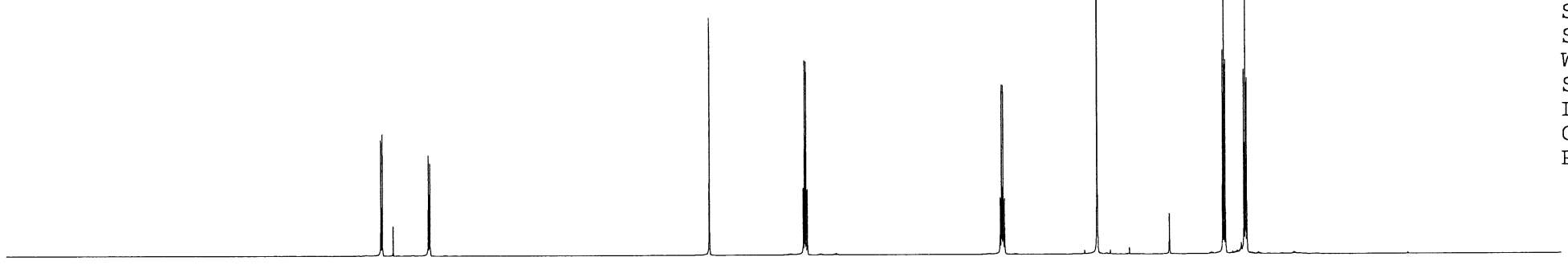

$700.0070162 \mathrm{MHz}$

WDW

0

HB $\mathrm{Hz}$

$P C$

1.00

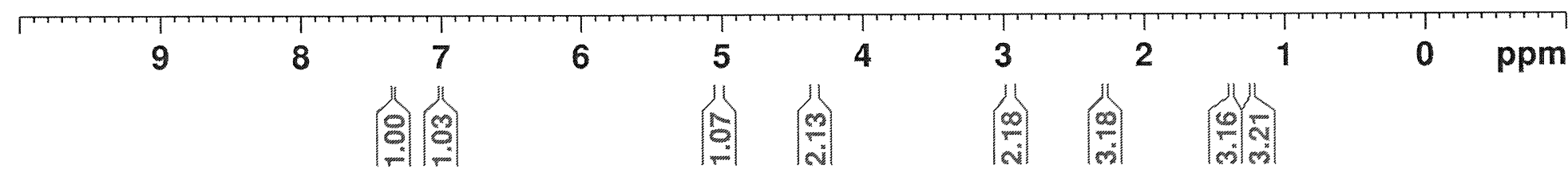




i

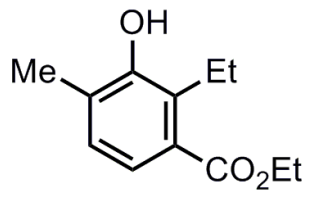

57
Current Data Parameters NAME

EXPNO

PROCNO

F2 - Acquisition Parameters

Date_

Time

INSTRUM

ROBHD

PULPROG

TD

SOLVENT

NS

DS

SWH

FIDRES

$\mathrm{AQ}$
$\mathrm{RG}$
$\mathrm{DW}$

RW

$\mathrm{DE}$

TE

D 11
TD 0
SFO 1

SFO1

NUC1

$\mathrm{P} 1$

PLW1

$\mathrm{SFO} 2$

NUC2

CPDPRG [2

PCPD2

(1) 2

PLW12

F2 - Processing parameters

SI 131072

SF $\quad 176.0169002 \mathrm{MHz}$

WDW

SSB

LB

GB
PC

$$
17.03 \mathrm{~h}
$$

spect

zgpg30

65536

35

$42613.637 \mathrm{~Hz}$

$1.300465 \mathrm{~Hz}$

$0.7689557 \mathrm{sec}$

11.733 use

18.00 usec $303.2 \mathrm{~K}$

$2.00000000 \mathrm{sec}$

$0.03000000 \mathrm{sec}$

$176.0362620 \mathrm{MHz}$ 130 $29.00000000 \mathrm{~W}$ $700.0098000 \mathrm{MHz}$ waltz16

65.00 use

$13.00000000 \mathrm{~W}$

$0.27768999 \mathrm{~W}$
$1.00 \mathrm{~Hz}$

1.40

\begin{tabular}{lrrrrrrrrrr}
\hline 200 & 180 & 160 & 140 & 120 & 100 & 80 & 60 & 40 & 20 & $\mathrm{ppm}$
\end{tabular}



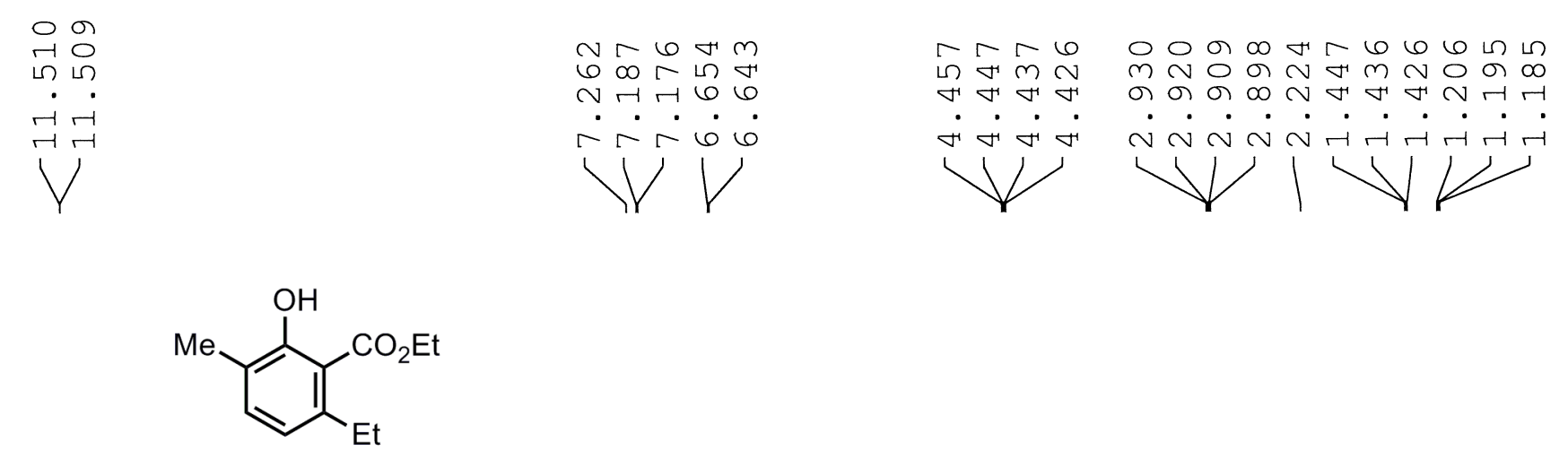

58

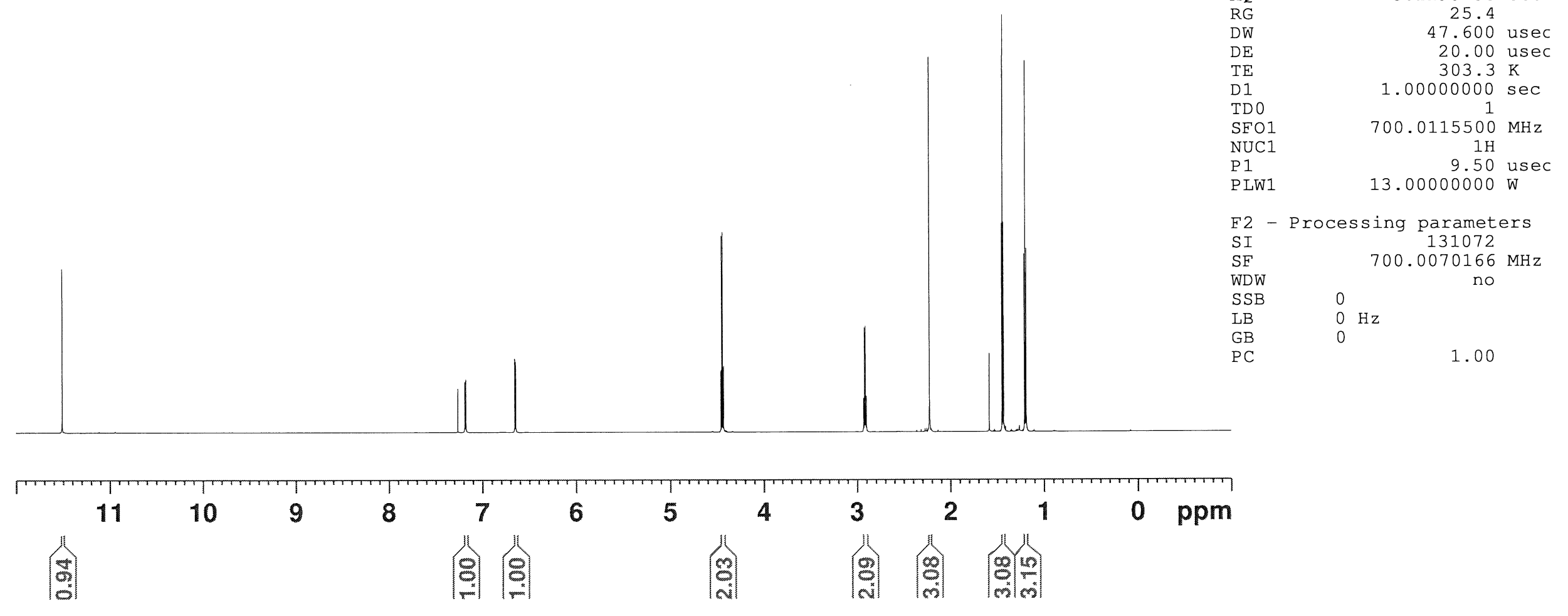




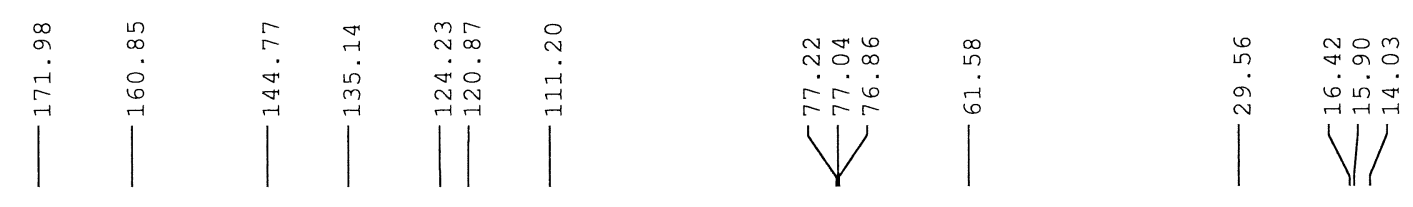
Current Data Parameters
NAME

EXPNO

PROCNO

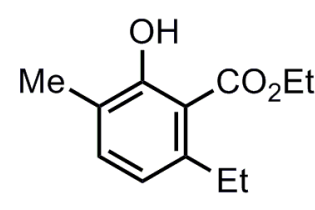

58

F2 - Acquisition Parameters

Date_ 20190821

16.20 h

INSTRUM

PUTPROG

spect

SOLVENT

NS

DS

SWH

FIDRES

AQ
RG

$D W$

$\mathrm{DE}$

1

DI

D11

SFO1

SFO1

P1

PLW1

$\mathrm{SFO} 2$

NUC2

CPDPRG [2

PCPD2

PLW2

PLW12

zgpg30

65536

$\mathrm{CDC} 13$
17

4
$42613.637 \mathrm{~Hz}$

$42613.637 \mathrm{~Hz}$
$1.300465 \mathrm{~Hz}$

203
11.733

.733 usec 18.00 use

$2.00000000 \mathrm{sec}$

$0.03000000 \mathrm{sec}$

1

$176.0362620 \mathrm{MHz}$ $13 \mathrm{C}$

$29.00000000 \mathrm{~W}$

$700.0098000 \mathrm{MHz}$

waltz16

altz16

$13.00000000 \mathrm{~W}$

$0.27768999 \mathrm{~W}$

$0.13982999 \mathrm{~W}$

F2 - Processing parameters

SI 131072

SF $\quad 176.0169002 \mathrm{MHz}$

WDW

SSB 0

EM
$1.00 \mathrm{~Hz}$
1.40

GB

PC 


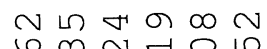
nO $\therefore-r \dot{\theta} \dot{0}$ $11 /$

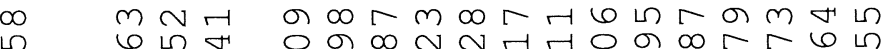

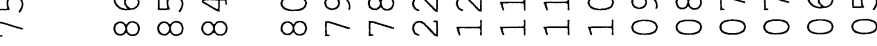

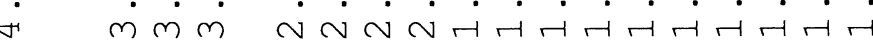

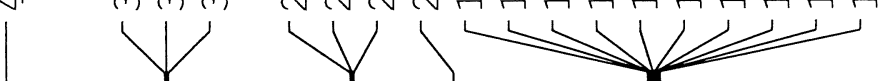

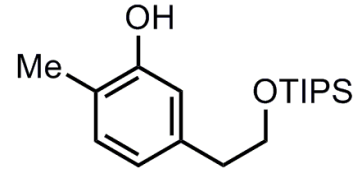

59 Current Data Parameters
NAME

EXPNO

PROCNO

F2 - Acquisition Parameters

Date_ 20191007

Time

$13.44 \mathrm{~h}$

INSTRUM

PROBHD

PULPROG

TD

SOLVENT

NS

DS

SWH

FIDRES

AQ

RG

DW

$\mathrm{DE}$

$\mathrm{TE}$

TDO

$\mathrm{SFO} 1$

NUC1

$\mathrm{PO}$

P 1
PLW1

2151340_0001 (

$\mathrm{zg} 30$
65536

$\mathrm{CDCl}$

16

$10504.202 \mathrm{~Hz}$

$0.320563 \mathrm{~Hz}$

$3.1195135 \mathrm{sec}$

47.600 usec

20.00 usec
$303.2 \mathrm{~K}$

$1.00000000 \mathrm{sec}$

$700.0115500 \mathrm{MHz}$

$1 \mathrm{H}$

3.17 usec

9.50 usec

$13.00000000 \mathrm{~W}$

F2 - Processing parameters

SI 131072

SF $\quad 700.0070151 \mathrm{MHz}$

WDW

WDW

LB

$\mathrm{PC}$

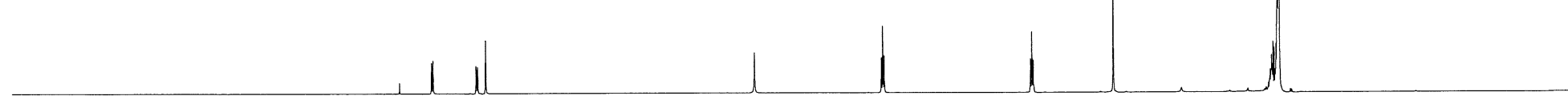

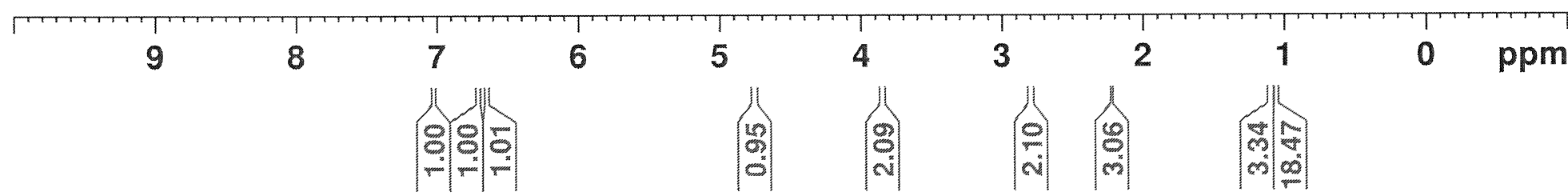




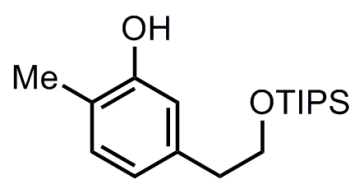

59
Current Data Parameters

NAME

EXPNO

PROCNO

F2 - Acquisition Parameters

Date_ 20191007

Time

$13.49 \mathrm{~h}$

INSTRUM

PROBHD

PUIPROG

TD

SOLVENT

SWH

FIDRES

AQ

RG

DW

$\mathrm{DE}$

11

11

TDO

NUC1

$\mathrm{PO}$

$\mathrm{P} 1$

PLW1

$\mathrm{SFO} 2$

NUC2

CPDPRG [ 2

PCPD2

PLW2

PLW12

PLW13

spect

0001 (

zgpg 30

CDCl 3

$\mathrm{CDCl}$

$42613.637 \mathrm{~Hz}$

$1.300465 \mathrm{~Hz}$

$0.7689557 \mathrm{sec}$

11.733 usec

18.00 usec $303.2 \mathrm{~K}$

$2.00000000 \mathrm{sec}$ $0.03000000 \mathrm{sec}$ 1

$176.0362620 \mathrm{MHz}$ $13 \mathrm{C}$

3.75 usec
11.25 usec

$29.00000000 \mathrm{~W}$

$700.0098000 \mathrm{MHz}$

waltz16

65.00 usec

$13.00000000 \mathrm{~W}$

$0.27768999 \mathrm{~W}$

$0.13982999 \mathrm{~W}$

2 - Processing parameters

SI 131072

SF $\quad 176.0169002 \mathrm{MHz}$

WDW

SSB

$I B$

$\mathrm{GB}$
$\mathrm{PC}$

$\mathrm{EM}$
0
$1.00 \mathrm{~Hz}$
0
1.0

\begin{tabular}{|c|c|c|c|c|c|c|c|c|c|}
\hline 200 & 180 & 160 & 140 & 120 & 100 & 80 & 60 & 40 & 20 \\
\hline
\end{tabular}


の $\infty \cdots \omega \sim \pi m a$

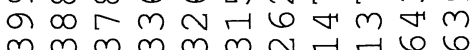
$m m m m m N-\pi 6$

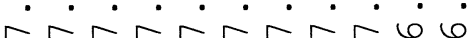
$\underbrace{5}$<smiles>CCc1cc(O)cc(CCOC(C)(F)F)c1P</smiles>

60

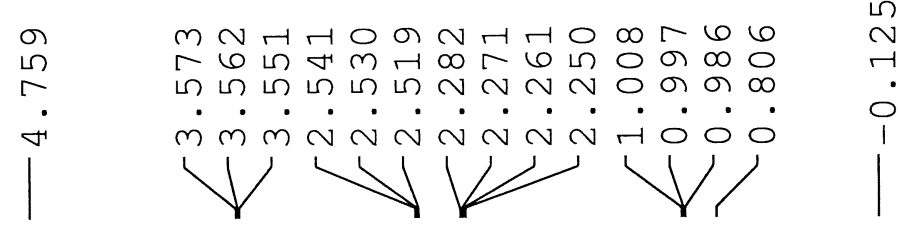

Current Data Parameters NAME

EXPNO

PROCNO

F2 - Acquisition Parameters

Date_ 20190326

Time

$13.46 \mathrm{~h}$

INSTRUM

ROBHD

PULPROG

TD

SOLVENT

NS

DS

SWH

FIDRES

AQ

RG

DW

DE

TE

D1

TDO

NEO1

PLW1

2151340_0001)

$\mathrm{zg} 30$
65536

16

$10504.202 \mathrm{~Hz}$

$0.320563 \mathrm{~Hz}$

$3.1195135 \mathrm{sec}$ 6.35

47.600 usec

10.00 usec

$303.2 \mathrm{~K}$

$1.00000000 \mathrm{sec}$

1

$$
1 \mathrm{H}
$$

9.50 usec

$13.00000000 \mathrm{~W}$

F2 - Processing parameters

SI 131072

SF $\quad 700.0070161 \mathrm{MHz}$

WDW

SSB $\quad 0$

LB $\quad 0 \mathrm{~Hz}$

1.00

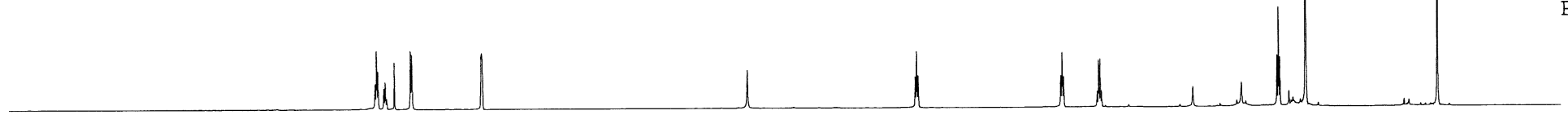

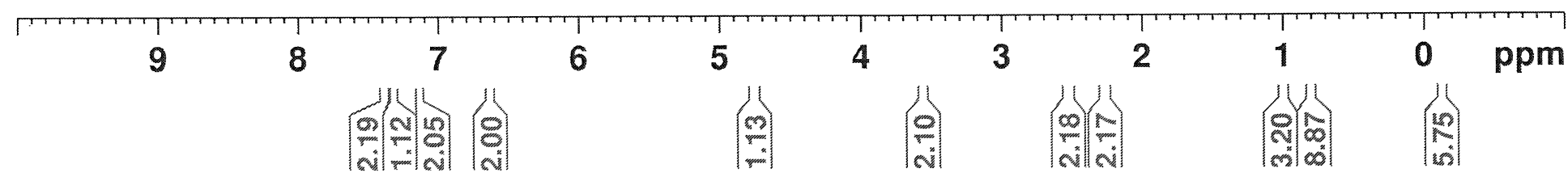



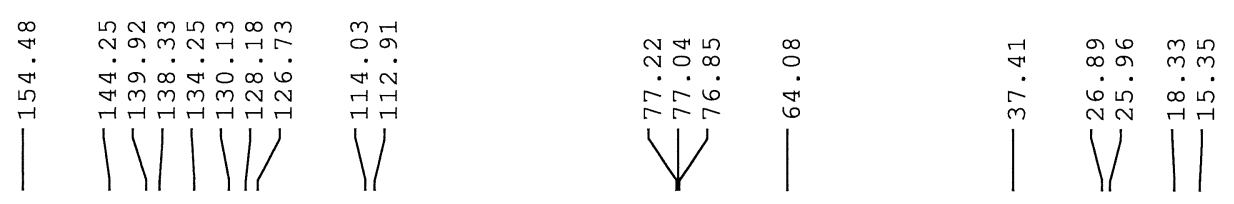

\begin{tabular}{l}
0 \\
\multirow{1}{*}{} \\
1
\end{tabular}<smiles></smiles>

Current Data Parameters NAME

EXPNO $\mathrm{XZ}-3-108$ PROCNO

F2 - Acquisition Parameter Date_ 20190326

Time

INSTRUM

P ROBHD

PULPROG

$13.53 \mathrm{~h}$

TD

SOLVENT

NS

NS

SWH

FIDRES

$\mathrm{AQ}$

RG

$D W$

DE

D1

D 11

DI1

SFO1

$\mathrm{SFO1}$

P 1

$\mathrm{P} 1$

P LW1

$\mathrm{SFO} 2$

NUC 2

CPDPRG [ 2

PCPD 2

P LW2

P LW1 2

spect

0001

z gpg30

$\mathrm{CDC} 13$
36
4

$42613.637 \mathrm{~Hz}$

1.300465 Hz $0.7689557 \mathrm{sec}$ 203

11.733 usec 18.00 usec $303.2 \mathrm{~K}$

$2.00000000 \mathrm{sec}$ $0.03000000 \mathrm{sec}$ $176.0362620 \mathrm{MHz}$ $13 \mathrm{C}$

11.25 usec $29.00000000 \mathrm{~W}$ $700.0098000 \mathrm{MHz}$

$$
\text { waltz } 16
$$

65.00 user

$13.00000000 \mathrm{~W}$

$0.27768999 \mathrm{~W}$

$0.13982999 \mathrm{~W}$

F2 - Processing parameters

F2 Processing

131072

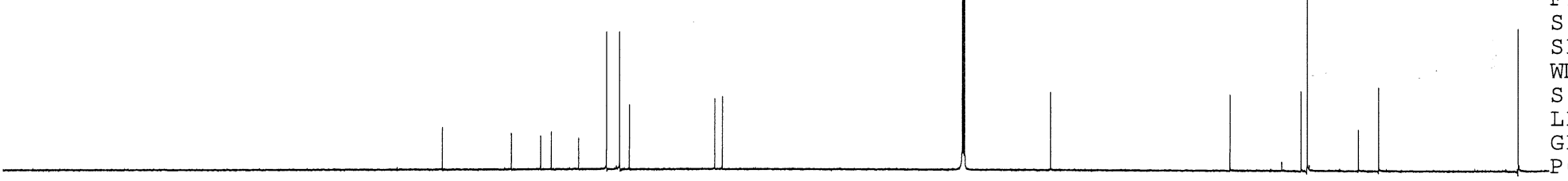

$\quad 176.0169002 \mathrm{MHz}$ EDW

S SB

LB

PC

$1.00 \mathrm{~Hz}$

1.40

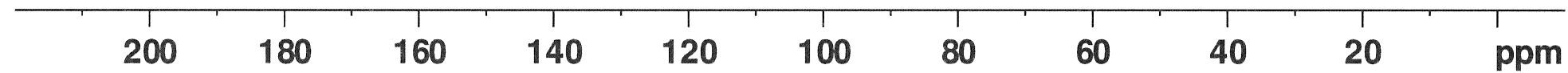




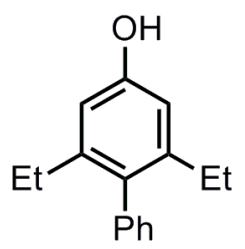

61
Current Data Parameters

NAME

EXPNO

PROCNO $\mathrm{XZ}-3-218$

F2 - Acquisition Parameter Date_ 20190725 Time

INSTRUM

PROBHD

PULPROG $11.07 \mathrm{~h}$

TD

z151340_0001

SOLVENT

NS

DS

FIDRES

$\mathrm{AO}$

RG

DW

$\mathrm{DE}$

D 1

TD 0

$\mathrm{SFO} 1$

NUC 1

$\mathrm{P} 1$

P LW1

zg30

$\mathrm{CDCl} 3$ 16

$10504.202 \mathrm{~Hz}$ $0.320563 \mathrm{~Hz}$ $3.1195135 \mathrm{sec}$ 22.6

47.600 usec 20.00 usec $303.4 \mathrm{~K}$

$1.00000000 \mathrm{sec}$ $700.0115500 \mathrm{MHz}$ $1 \mathrm{H}$
$.50 \mathrm{usec}$

$13.00000000 \mathrm{~W}$

F2 - Processing parameters SI $\quad 131072$

SF $\quad 700.0070168 \mathrm{MHz}$ WDW

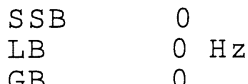

GB

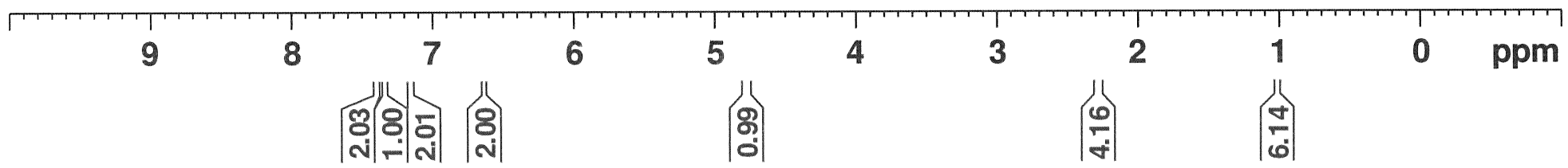


<smiles>CCc1cc(O)cc(CC)c1-c1ccccc1</smiles>

61
Current Data Parameters NAME

EXPNO

PROCNO

F2 - Acquisition Parameters

Date 20190725

Date_

11.11

Time

PROBHD

PULPROG

TD

SOLVENT

NS

SWH

FIDRES

$\mathrm{AQ}$
$\mathrm{RG}$

DW

DE

TE

D11
TD 0
SFO1

NUC1

1

PLW1

$\mathrm{SFO} 2$

NUC2

CPDPRG [2

PCPD 2

PIV1

PLW12

spect

0001

zgpg30
65536

$\mathrm{CDCl} 3$ 17
4

$42613.637 \mathrm{~Hz}$

$1.300465 \mathrm{~Hz}$

$0.7689557 \mathrm{sec}$ 203

11.733 usec 18.00 usec

$2.00000000 \mathrm{sec}$

$0.03000000 \mathrm{sec}$

$176.0362620 \mathrm{MHz}$ $13 \mathrm{C}$ 11.25 use $29.00000000 \mathrm{~W}$ $700.0098000 \mathrm{MHz}$ $1 \mathrm{H}$
walt $z 16$ 65.00 usec

$13.00000000 \mathrm{~W}$ $0.27768999 \mathrm{~W}$ $0.13982999 \mathrm{~W}$

F2 - Processing parameters

SI Processing parameters 131072

SF $\quad 176.0169002 \mathrm{MHz}$

SSB

LB

$\mathrm{GB}$
$\mathrm{PC}$

EM

$1.00 \mathrm{~Hz}$

1.40

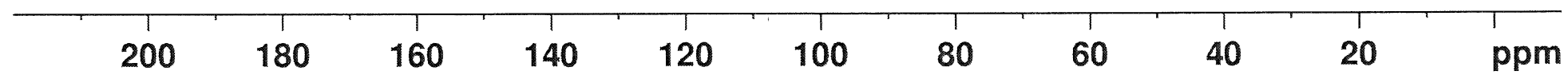


$m m \sim m-6 \sim 66$ H

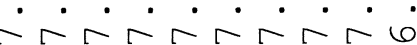

$\rightarrow \infty$<smiles>CCc1cc(-c2ccccc2)c(CC)cc1O</smiles>

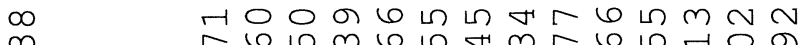

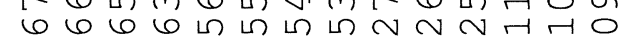

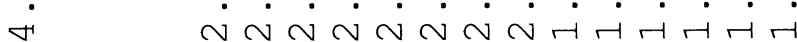

N N N N N
Current Data Parameters

NAME

EXPNO

PROCNO

F2 - Acquisition Parameters

Date__ 20190415

Time

$14.49 \mathrm{~h}$

INSTRUM

PROBHD

PULPROG

TD

SOLVEN'

NS

DS

SWH

FIDRE

AQ

RW

DE

TE

D1

TDO

SFO1

P 1

PLW1

spect

spect

zg30

zg30
65536

$\mathrm{CDCl} 3$

16

$10504.202 \mathrm{~Hz}$

$0.320563 \mathrm{~Hz}$

$3.1195135 \mathrm{sec}$ 6.35

47.600 usec

10.00 usec

$303.2 \mathrm{~K}$

$1.00000000 \mathrm{sec}$

1

$1 \mathrm{H}$
9.50 usec

$13.00000000 \mathrm{~W}$

F2 - Processing parameters SI 131072 SF $\quad 700.0070141 \mathrm{MHz}$

WTW

$S S B$

SSB

LB

GB
PC

0

$0 \mathrm{~Hz}$

no

1.00

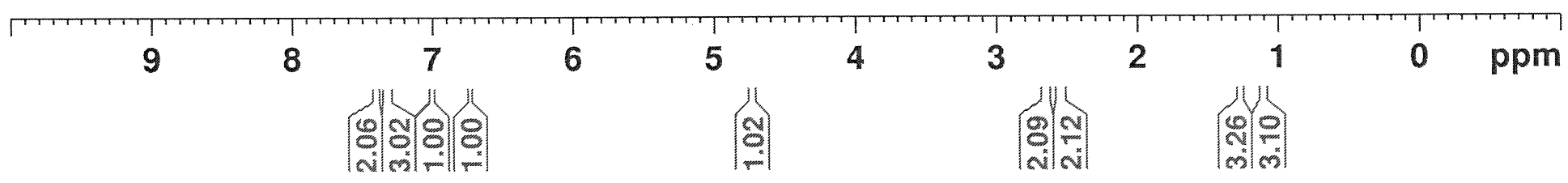




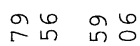
กับ 111<smiles>CCc1cc(O)c(-c2ccccc2)cc1CC</smiles>

62
Current Data Parameters NAME

EXPNO

PROCNO

F2 - Acquisition Parameters

Date__ 20190415

Time-

$14.53 \mathrm{~h}$

INSTRUM

PROBHD

PULPROG

TD

SOLVENT

NS

DS

SWH

FIDRES

$A Q$
$R G$

DW

$\mathrm{DE}$

E

11

TD 0

$\mathrm{SFO1}$

NUC1

1 1

PLW1

$\mathrm{SFO} 2$

NUC2

CPDPRG [ 2

PCPD 2

PLW2

P LW12

F2 - Processing parameters

SI 131072

SF $176.0169002 \mathrm{MH}$

WDW

SSB

LB

GB
PC
2151340 spect

zgpg 30

21

$42613.637 \mathrm{~Hz}$

$1.300465 \mathrm{~Hz}$

$0.7689557 \mathrm{sec}$

11.733 usec

18.00 usec $303.2 \mathrm{~K}$

$2.00000000 \mathrm{sec}$

$0.03000000 \mathrm{sec}$

$176.0362620^{1} \mathrm{MHz}$ $13 \mathrm{C}$

$29.00000000 \mathrm{~W}$ $700.0098000 \mathrm{MHz}$

waltz16

65.00 usec

$13.00000000 \mathrm{~W}$

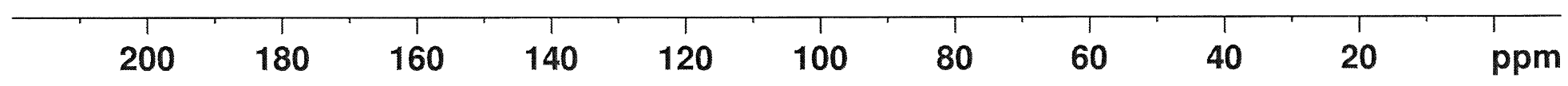


ம 가ㅁㅏㅏ 아 -

$\longrightarrow$ L<smiles>CCc1cc(O)c(Br)cc1-c1ccccc1</smiles>

63
Current Data Parameters NAME

EXPNO

PROCNO

$\mathrm{xz}-2-303$

F2 - Acquisition Parameter

Date_ 20181120

Time $\quad 10.08 \mathrm{~h}$

INSTRUM spect

PROBHD Z151340_0001

PULPROG Z930

TD 65536

SOLVENT CDCl3

NS

16

FIDRES

AQ

RG

DW

$\mathrm{DE}$

TE

DI

$\mathrm{SFO} 1$

NUC1

$\mathrm{P} 1$

P LW1

$10504.202 \mathrm{~Hz}$

$0.320563 \mathrm{~Hz}$

$3.1195135 \mathrm{sec}$ 5.6

47.600 usec

10.00 usec

$303.2 \mathrm{~K}$

$1.00000000 \mathrm{sec}$

$700.0115500 \mathrm{MHz}$ $1 \mathrm{H}$

$$
9.1
$$

13.00000000 us

F2 - Processing parameters SI $\quad 131072$

SF $\quad 700.0070173 \mathrm{MHz}$

SSB

LB

GB

${ }_{0}^{0} \mathrm{~Hz}$

.00
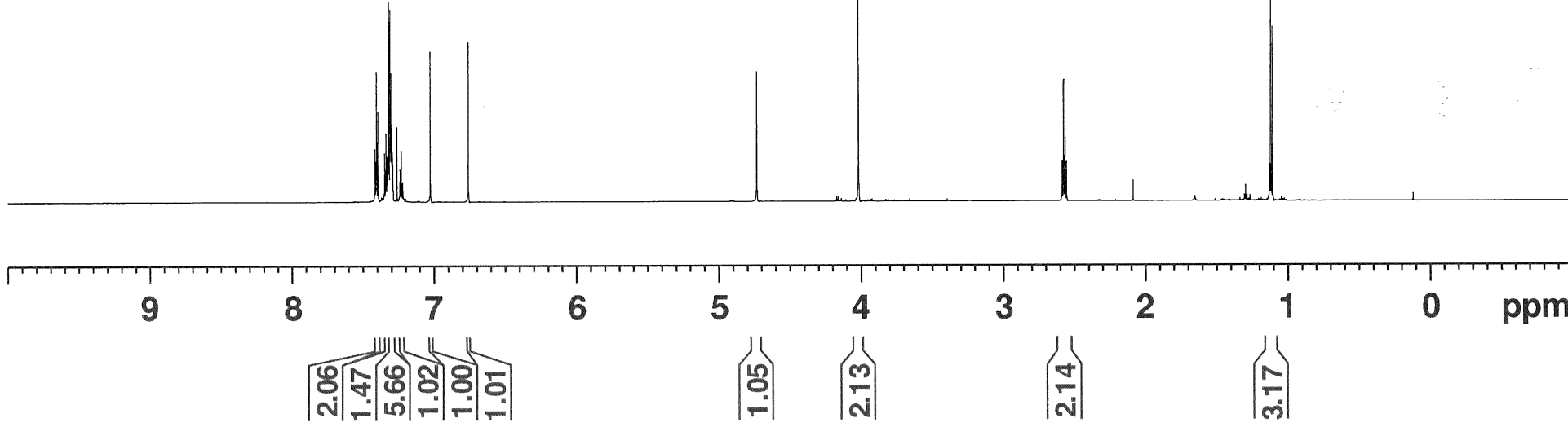
<smiles>CCc1cc(O)c(Br)cc1-c1ccccc1</smiles>

63
Current Data Parameters NAME

EXPNO

PROCNO

$$
1
$$

F2 - Acquisition Parameter

Date_ 20181120

Time

$10.13 \mathrm{~h}$

INSTRUM

PROBHD Z151340 0001

PULPROG Zgpg30

SOLVENT

NS

DS

FIDRE

AQ

RG

DW

DE

D 1

D 11

TD 0

SFO1

$\mathrm{P} 1$

PLW1

$\mathrm{SFO} 2$

NUC2

CPDPRG [ 2

PCPD 2

P LW2

P LW1 2

PLW13

z gpg30

$\mathrm{CDCl} 3$ 40
4

$42613.637 \mathrm{~Hz}$

$1.300465 \mathrm{~Hz}$ $0.7689557 \mathrm{sec}$ 203

11.733 usec 18.00 usec $303.2 \mathrm{~K}$

$2.00000000 \mathrm{sec}$ $0.03000000 \mathrm{sec}$ $176.0362620 \mathrm{MHz}$ $13 \mathrm{C}$

11.25 usec $29.00000000 \mathrm{~W}$ $700.0098000 \mathrm{MHz}$

$$
\text { waltzi } 16
$$$$
65.00 \text { user }
$$

$13.00000000 \mathrm{~W}$

$0.27768999 \mathrm{~W}$

$0.13982999 \mathrm{~W}$

F2 - Processing parameters
SI
131072

SF $\quad 176.0169002 \mathrm{MHz}$

WDW $\quad 176.0169002$

SSB $\quad 0$

$\mathrm{LB}$

GB
P C

0 
几

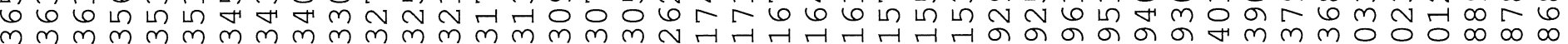

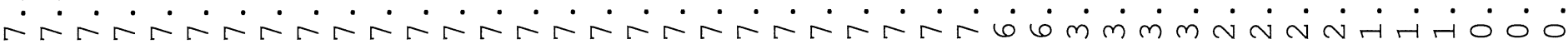

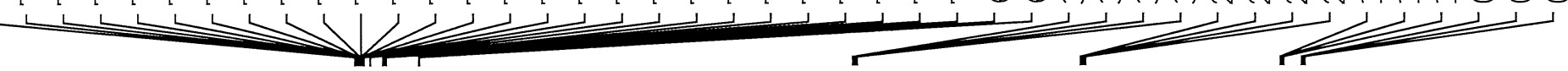

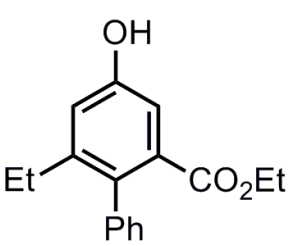

64

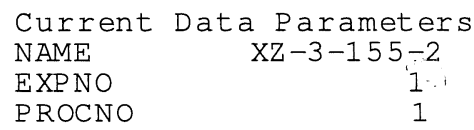

F2 - Acquisition Parameter Date_ 20190822

Time $13.39 \mathrm{~h}$

INSTRUM spect

PROBHD Z151340_0001

PULPROG $\quad \mathrm{zg} 30$

TD 65536

SOLVENT CDCl3

NS

DS

SWH
FIDRES

AQ

RG

DW

$\mathrm{DE}$

TE

TDO

SFO1

NUC 1

P 1

P LW1

F2 - Processing parameters

SI Processing paramet

SF $\quad 700.0070164 \mathrm{MH}$

WDW

SSB

$\mathrm{LB}$
$\mathrm{GB}$

$\mathrm{PC}$

0

$\mathrm{O}^{\mathrm{Hz}}$

$10504.202 \mathrm{~Hz}$ $0.320563 \mathrm{~Hz}$ $3.1195135 \mathrm{sec}$

47.600 usec 20.00 usec $303.4 \mathrm{~K}$

$1.00000000 \mathrm{sec}$ $700.0115500 \mathrm{MHz}$ 11

$13.00000000 \mathrm{~W}$

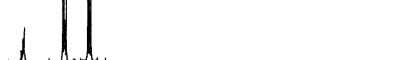

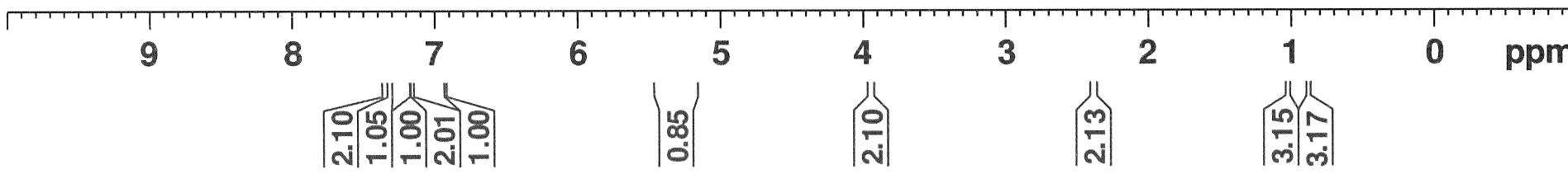




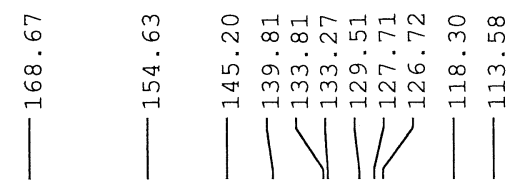

$\overbrace{\mathrm{Ph}}^{\mathrm{OH}}$

64

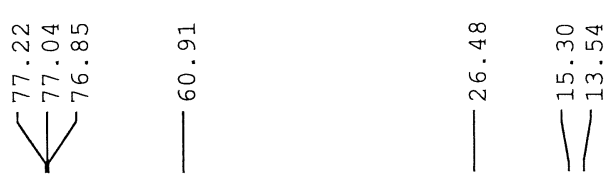

Current Data Parameters

NAME

EXPNO

PROCNO

$\mathrm{Xz}-3-155-2$

- Acquisition Parameters

Date__ 20190822

13.50

INSTRUM spect

Z151340_0001

PULPROG zgpg30

TD 65536

OLVENT CDCl3

NS

DS

FIDRES

$\mathrm{AQ}$

RG

DW

$\mathrm{DE}$

TE

DI

11

TD 0

SFO1

$\mathrm{NUC1}$
$\mathrm{P} 1$

PLW1

$\mathrm{SFO} 2$

NUC2

CPDPRG [ 2

PCPD2

PLW2

P LW12
PLW13

121

$42613.637 \mathrm{~Hz}$

$1.300465 \mathrm{~Hz}$

$0.7689557 \mathrm{sec}$ 203

11.733 usec 18.00 usec $303.2 \mathrm{~K}$

$2.00000000 \mathrm{sec}$

$0.03000000 \mathrm{sec}$

$176.0362620^{1} \mathrm{MHz}$ $13 \mathrm{C}$

11.25 usec $29.00000000 \mathrm{~W}$ $700.0098000 \mathrm{MHz}$ $1 \mathrm{H}$
waltz 16

65.00 usec

$13.00000000 \mathrm{~W}$

$0.27768999 \mathrm{~W}$

$0.13982999 \mathrm{~W}$

F2 - Processing parameters

SI Processing parameter

SI $\quad 131072$

$176.0169002 \mathrm{MHz}$

SSB EM

GB

$1.00 \mathrm{~Hz}$

1.40

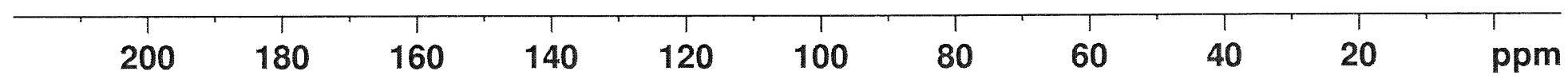


NH이의 मु arrararar 6 6 6 $\longrightarrow 3$

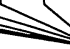<smiles>CCc1cc(O)cc([N+](=O)[O-])c1-c1ccccc1</smiles>

65

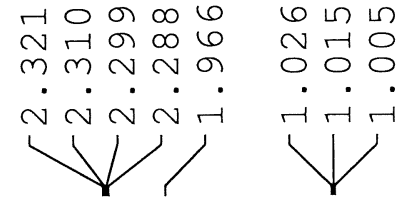

Current Data Parameters

NAME

EXPNO

PROCNO

$\mathrm{Xz}-3-267$

F2 - Acquisition Parameters

Date__ 20190805

Time $17.31 \mathrm{~h}$

INSTRUM spect

PROBHD Z151340_0001) (

PULPROG $\quad$ zg30

$\begin{array}{lr}\text { PULPROG } & \text { Zg30 } \\ \text { TD } & 65536\end{array}$

SOLVENT CDCl3

NS

DS

FIDRES $\quad 0.320563 \mathrm{~Hz}$

AQ $\quad 3.1195135 \mathrm{sec}$

RG

DW

$\mathrm{DE}$

TE

D1

TD 0

NUC1

P1

PLW1

28.5

47.600 usec

20.00 usec

$1.00000000 \mathrm{~K}$

1.00000000 sec

$700.0115500 \mathrm{MHz}$

$1 \mathrm{H}$

9.50 usec

$13.00000000 \mathrm{~W}$

F2 - Processing parameters

SI 131072

SF $\quad 700.0070173 \mathrm{MHz}$

WDW

SSB

LB

GB

no

1.00
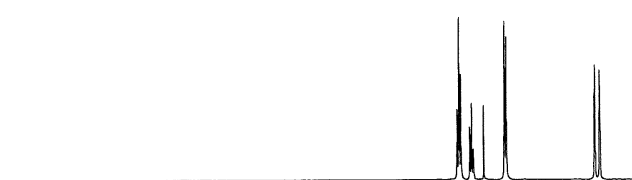

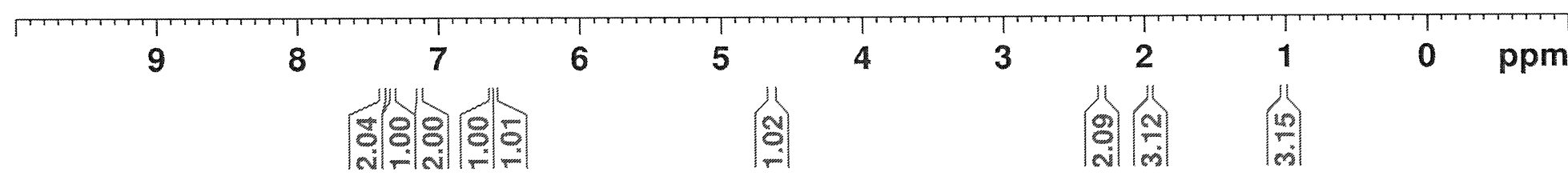



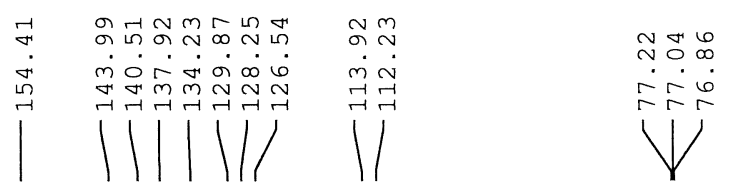

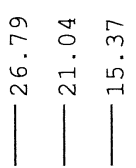

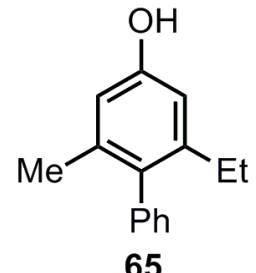

Current Data Parameters

NAME

$\mathrm{Xz}-3-267$

تXPNO

F2 - Acquisition Parameters

Date_ 20190805

Time $\quad 17.39 \mathrm{~h}$

INSTRUM spect

PROBHD Z151340_0001

PULPROG zgpg30

$\begin{array}{lr}\text { TD } & 65536\end{array}$

SOLVENT CDCl3

NS

DS

SWH

$\mathrm{AQ}$

RG

DW

$\mathrm{DE}$

$\mathrm{TE}$

D1

D 11

TDO

NUC1

P1

P 1
PLW1

$\mathrm{SFO} 2$

NUC2

CPDPRG [2

PCPD2

PIW2

PLW12

4
$42613.637 \mathrm{~Hz}$

$1.300465 \mathrm{~Hz}$

$0.7689557 \mathrm{sec}$

11.733 usec 18.00 usec $303.2 \mathrm{~K}$

$2.00000000 \mathrm{sec}$

$0.03000000 \mathrm{sec}$

$176.0362620^{1} \mathrm{MHz}$

$13 \mathrm{C}$
11.25 usec

$29.00000000 \mathrm{~W}$

$700.0098000 \mathrm{MHz}$

waltz 16

65.00 usec

$13.00000000 \mathrm{~W}$

$0.27768999 \mathrm{~W}$

$0.13982999 \mathrm{~W}$

F2 - Processing parameters

SI 131072

SF $\quad 176.0169002 \mathrm{MHz}$

SSB

$L B$

LB

0

$\mathrm{PC}$

EM
$1.00 \mathrm{~Hz}$
1.40

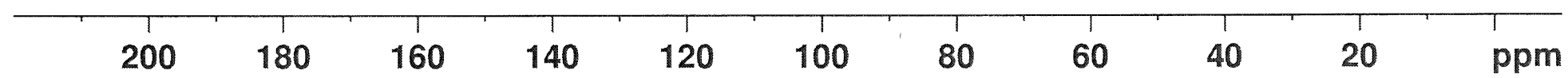




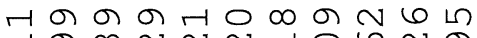
न irararararár

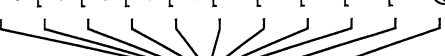

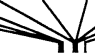

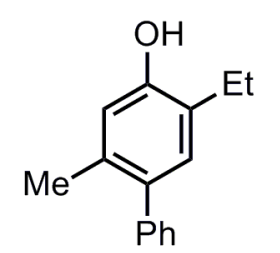

66

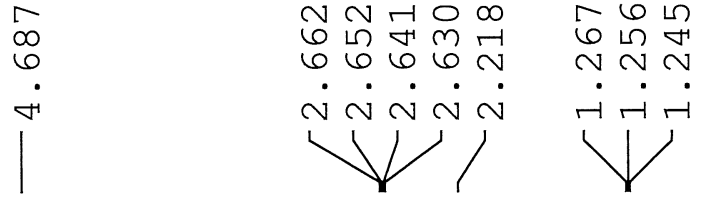

Current Data Parameters

NAME

EXPNO

PROCNO

$\mathrm{XZ}-3-112$

Acquisition Parameter

Date_ 20190822

Iime $17.19 \mathrm{~h}$

PROBHD Z151340_0001)

PULPROG zg30

TD 65536

SOIVENT

NS

DS

SWH

FIDRES

$\mathrm{AQ}$

RG

DW

$\mathrm{DE}$

TE

TD 0

$\mathrm{SFO} 1$

NUC1

P 1

$\mathrm{CDC} 13$
16

16
2

$10504.202 \mathrm{~Hz}$

$0.320563 \mathrm{~Hz}$

$3.1195135 \mathrm{sec}$ 28.5

47.600 usec 20.00 usec $303.3 \mathrm{~K}$

$1.00000000 \mathrm{sec}$

$700.0115500 \mathrm{MHz}$ $1 \mathrm{H}$

9.50 usec

$13.00000000 \mathrm{~W}$

F2 - Processing parameters SI 131072

SF $\quad 700.0070145 \mathrm{MHz}$

WDW

S SB

LB

GB

$0 \mathrm{~Hz}$

$\mathrm{OHz}$

no
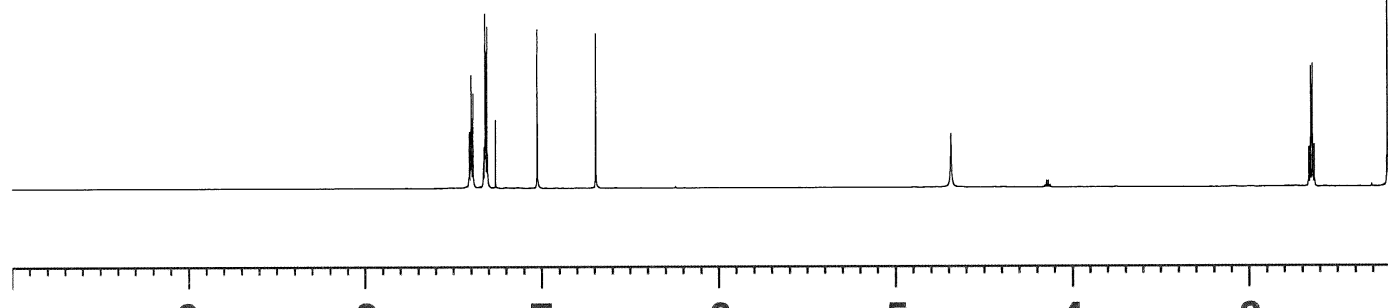

9

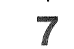

6

5

4

3

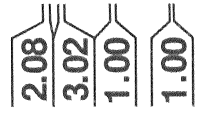

$\mid$

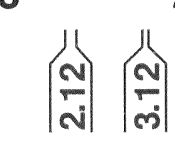

2

1

0

ppm

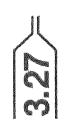




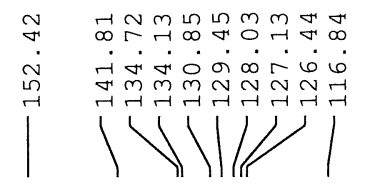

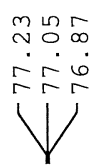

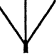

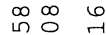

웅

11

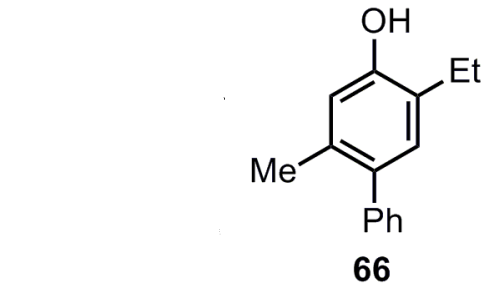

66

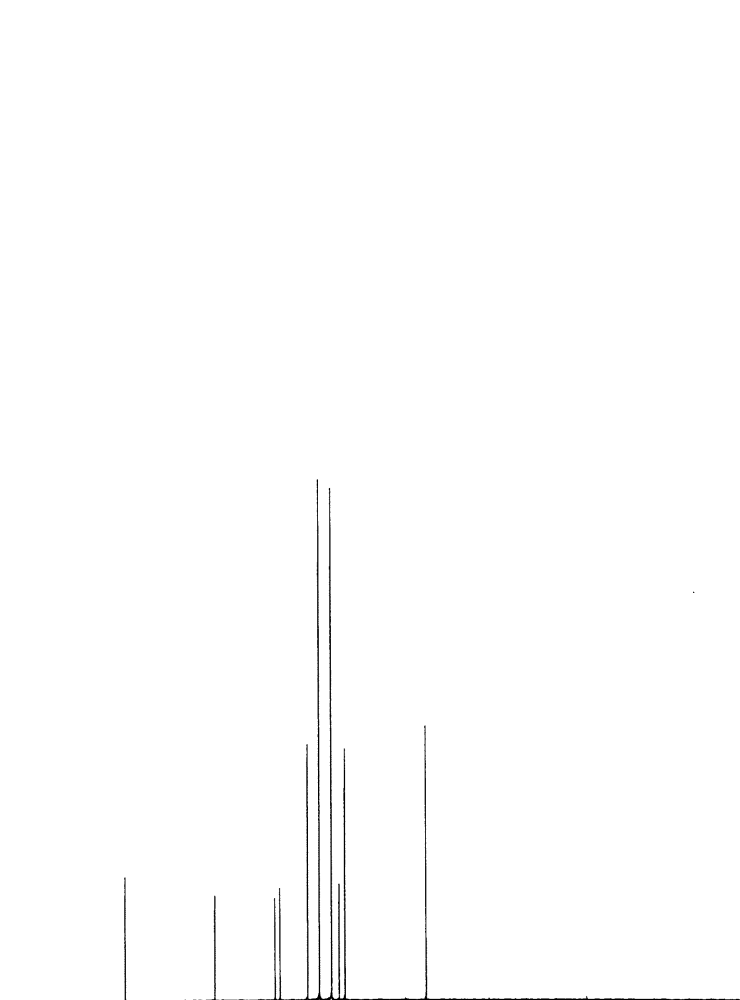

$\begin{array}{lr}\text { Current } & \text { Data } \\ \text { NAME } & \text { XZ-3-112 } \\ \text { EXPNO } & 2 \\ \text { PROCNO } & 1\end{array}$

F2 - Acquisition Parameters Date__ 20190325

Time

INSTRUM

Z151340 spect

PULPROG

TD

SOLVENT

NS

DS

SWH

FIDRES

AQ

DW

DW
$\mathrm{DE}$
$\mathrm{TE}$

D1

D11

TDO

NEO1

P1

PLW1

$\mathrm{SFO} 2$

NUC2

CPDPRG [ 2

PCPD 2

PLW2

PIVT12

PLW12

$151340 \_0001($
zgpg30
65536

$\mathrm{CDCl} 3$

35

$42613.637 \mathrm{~Hz}$

$1.300465 \mathrm{~Hz}$

$0.7689557 \mathrm{sec}$ 203

11.733 usec 18.00 usec 18.00 use

$2.00000000 \mathrm{sec}$

$0.0300000 \mathrm{sec}$

$176.0362620 \mathrm{MHz}$ $13 \mathrm{C}$

$29.00000000 \mathrm{~W}$ $700.0098000 \mathrm{MHz}$ waltz 16

65.00 use

$13.00000000 \mathrm{~W}$

$0.27768999 \mathrm{~W}$

$0.13982999 \mathrm{~W}$

F2 - Processing parameters

SI 131072

$176.0169002 \mathrm{MHz}$

LB

PB

0

$\mathrm{EM}$
$1.00 \mathrm{~Hz}$
1.40

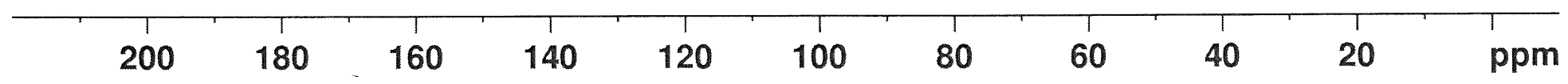




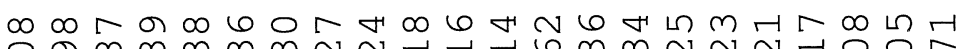

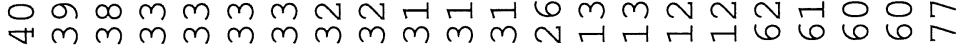
-rarrarrarararar

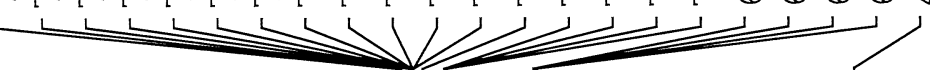

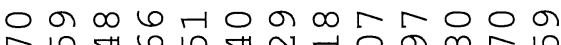
กำ ऽ $N$ ન

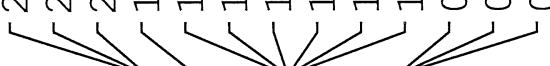

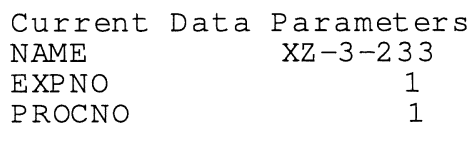

F2 - Acquisition Parameter Date_ 20190725

Time $11.17 \mathrm{~h}$

INSTRUM spect

PROBHD Z151340_0001

PULPROG zg30

TD 65536

SOLVENT CDCl3

NS 16

SWH

FIDRES

RG

DW

$\mathrm{DE}$

TE

TD 0

SFO1

NUC1

P 1

PLW1

$10504.202 \mathrm{~Hz}$

$0.320563 \mathrm{~Hz}$
$.1195135 \mathrm{sec}$ 25.4

47.600 usec

20.00 us ec

$303.3 \mathrm{~K}$

$1.00000000 \mathrm{sec}$

$700.0115500 \mathrm{MHz}$

$1 \mathrm{H}$
$9.50 \mathrm{usec}$

$13.00000000 \mathrm{~W}$

F2 - Processing parameters SI 131072

SF $\quad 700.0070167 \mathrm{MHz}$

WDW

S SB

$L B$

GB

${ }_{0}^{0} \mathrm{~Hz}$

1.00
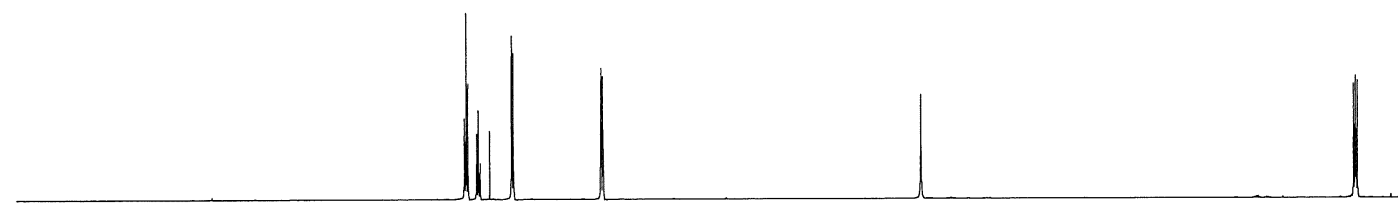

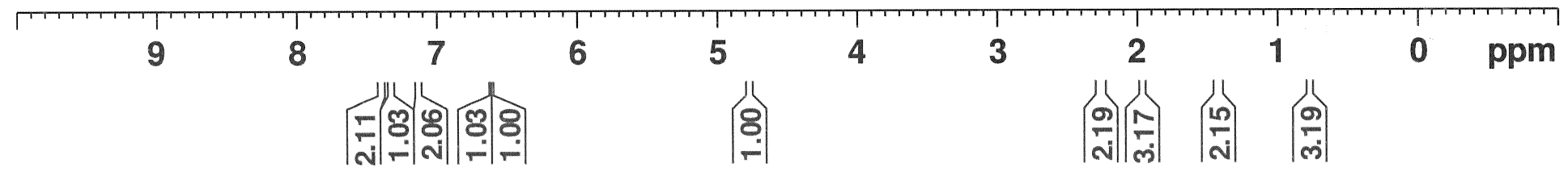




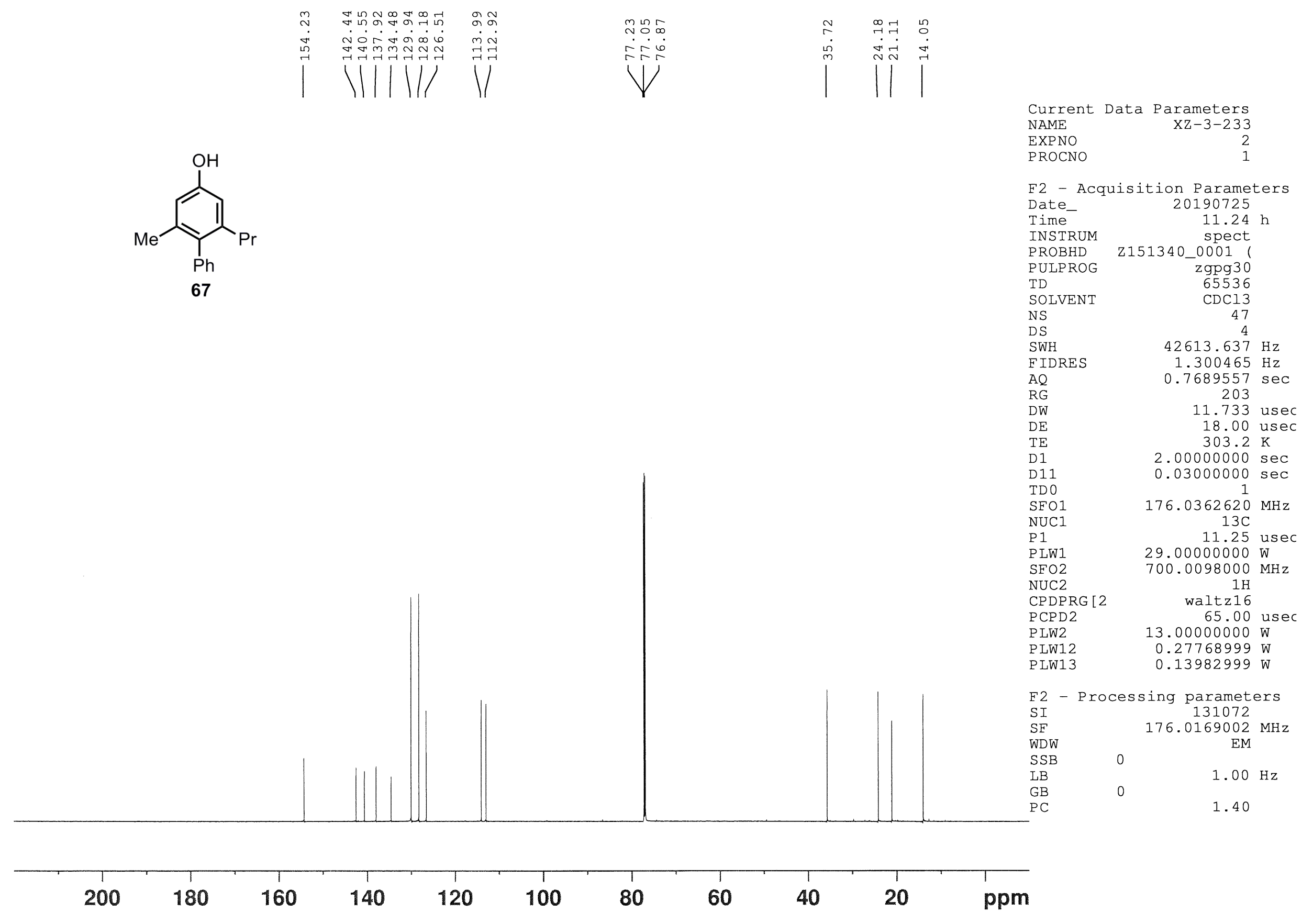




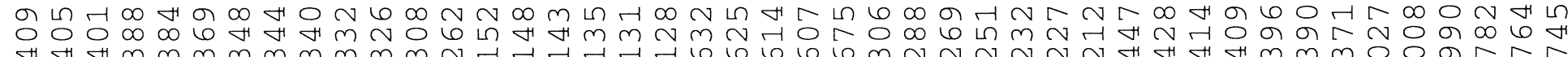
-
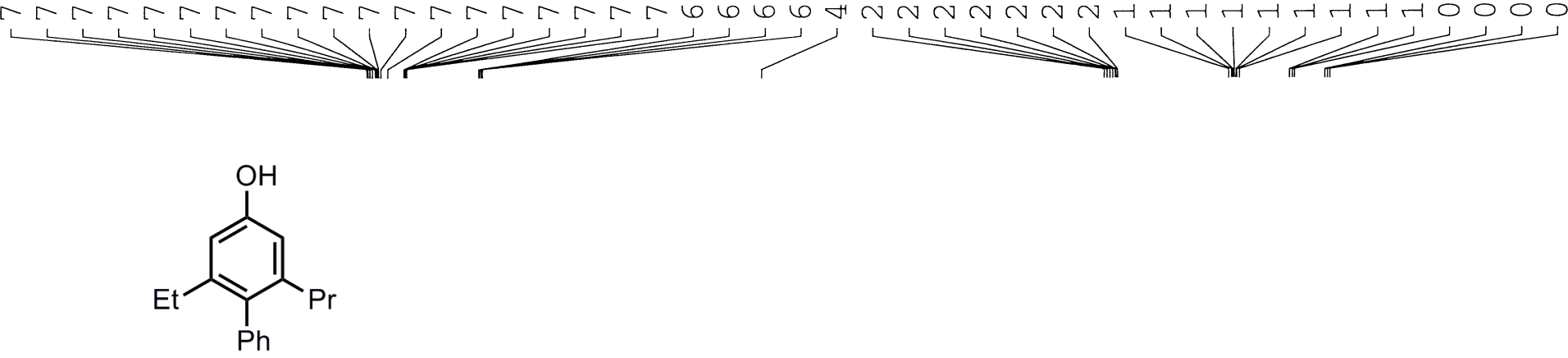

68
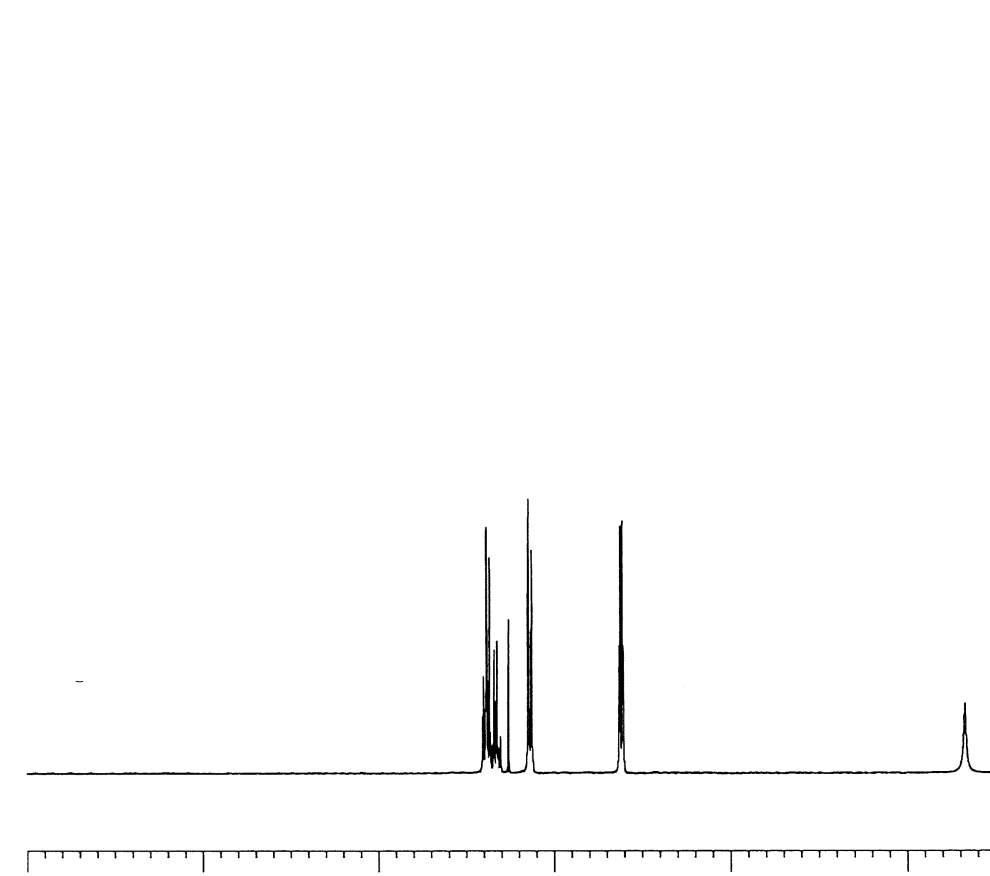

9

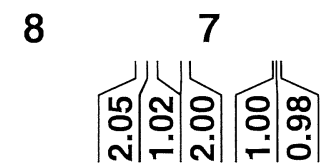

6

\section{NAME \\ EXPNO \\ Date \\ INSTRUM \\ PROBHD \\ PULPROG \\ TD \\ NS \\ SWH \\ FIDRES \\ $\mathrm{AQ}$ \\ RG \\ DW \\ TE \\ D1}

\section{$====$
NUC1}

P1

PL1

PL1W

SFO

SI

SF

SSB

LB

GB
PC
$\mathrm{XZ}-3-284$

20190906

11.56

zg30

65536

CDC13

32
2

$8278.146 \mathrm{~Hz}$

$0.126314 \mathrm{~Hz}$

3.9584243 ser

$$
\begin{array}{r}
322.5 \\
60.400
\end{array}
$$

60.400 us

6.50 usi

$1.00000000 \mathrm{se}$

CHANNEL $f 1======$ : $1 \mathrm{H}$

9.50 usi $-6.00 \mathrm{~dB}$ $40.90000153 \mathrm{~W}$ $400.1424710 \mathrm{MH}$ 400.1400081 MH: no
0 $0.00 \mathrm{~Hz}$ 1.00

1.00 
<smiles>CCc1cc(O)cc(P)c1-c1ccccc1</smiles>

68
SOTV

DS

SWH

FIDRES

$\mathrm{AQ}$

RG

$\mathrm{DW}$
$\mathrm{DE}$
$\mathrm{TE}$

D1

D11
TD0

$====$

NUC1

P1

PL1

PLIV

(1)

CPDPRG2

NUC2

PL2

PL12

PL13

PL2W

PL12W

PL13

SI

SF
WDW

SSB

LB

GB $x z-3-284$

20190906 12.14

spect

$\mathrm{BB}-1 \mathrm{H}$
$\mathrm{zgpg} 30$

65536

$\mathrm{CDCl} 3$ 232

$24154.590 \mathrm{~Hz}$ $0.368570 \mathrm{~Hz}$ $1.3566452 \mathrm{sec}$ 18390.4 20.700 use 6.50 use $298.2 \mathrm{~K}$

$2.70000005 \mathrm{sec}$ $0.03000000 \mathrm{sec}$

$==$ CHANNEL

$1=====$ $13 \mathrm{C}$ use 12.00 use $29.59002304 \mathrm{~W}$ $29.59002304 \mathrm{~W}$ $100.6263507 \mathrm{MHz}$ CHANNEL $\mathrm{f} 2======$ waltz16 $1 \mathrm{H}$
65.00 65.00 use $-6.00 \mathrm{~dB}$ $10.46 \mathrm{~dB}$ $12.50 \mathrm{~dB}$ $40.90000153 \mathrm{~W}$ $0.92410922 \mathrm{~W}$ $0.57772785 \mathrm{~W}$ $400.1416006 \mathrm{MHz}$ 131072
100.6152830 131072
$100.6152830 \mathrm{MHz}$ no
0

0
$0.00 \mathrm{~Hz}$
0

1.40

\begin{tabular}{|c|c|c|c|c|c|c|c|c|c|}
\hline 200 & $\begin{array}{c}1 \\
180\end{array}$ & 160 & 140 & 120 & 100 & 80 & 60 & 40 & 20 \\
\hline
\end{tabular}



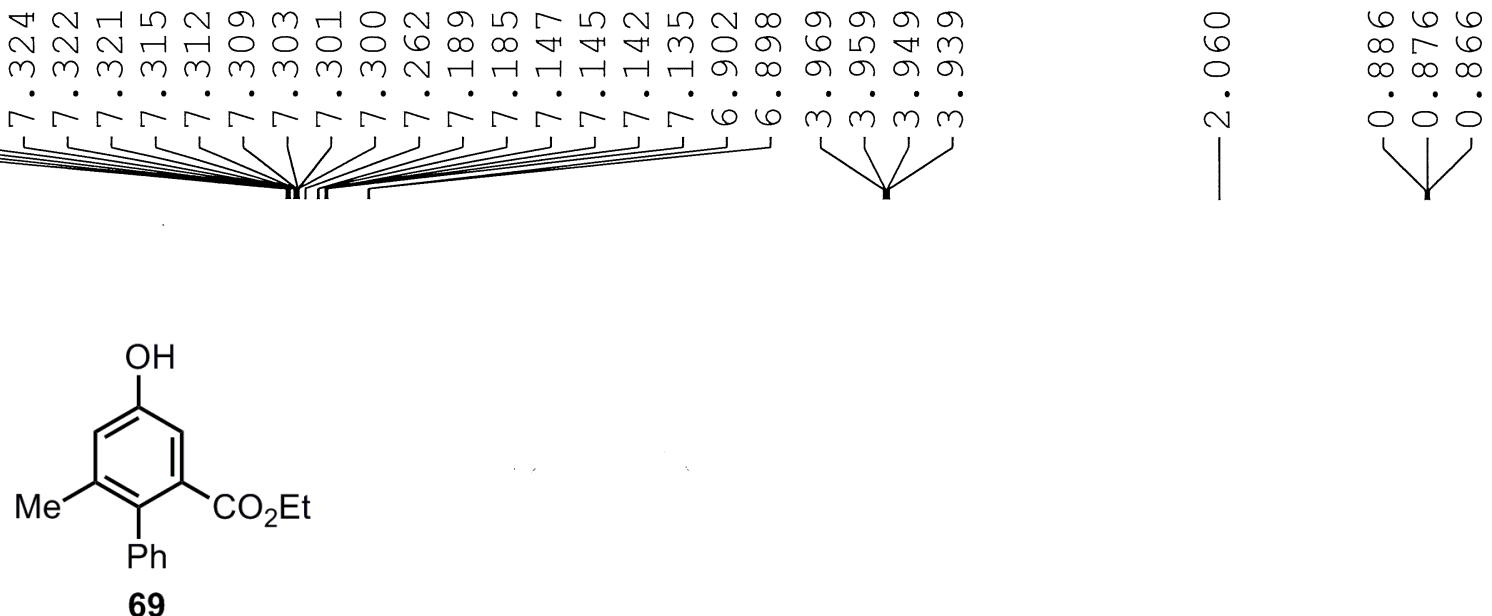
urrent Data Parameters
NAME
PROCNO
$\mathrm{XZ}-3-277$

F2 - Acquisition Parameters

Date_ 20190821

Time $15.54 \mathrm{~h}$

INSTRUM spect

PROBHD Z151340_0001 (

69 TD 65536

SOLVENT

SWH 210504.202

DSH

O. $0.320563 \mathrm{~Hz}$

AQ $\quad 3.1195135 \mathrm{sec}$

RG

DW $\quad 47.600$ usec

$\mathrm{DE} \quad 20.00$ usec $303.3 \mathrm{~K}$

D1 $1.00000000 \mathrm{sec}$

TD0

SFO1 $700.0115500 \mathrm{MHz}$

NUC1

P1

$1 \mathrm{H}$

PLW1

13.00000000 us

F2 - Processing parameters

SI 131072

SF $\quad 700.0070167 \mathrm{MHz}$

WSB

SSB

EM
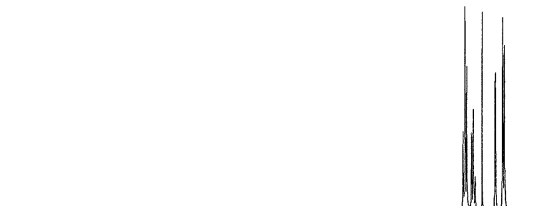

GB

$0.30 \mathrm{~Hz}$

1.00

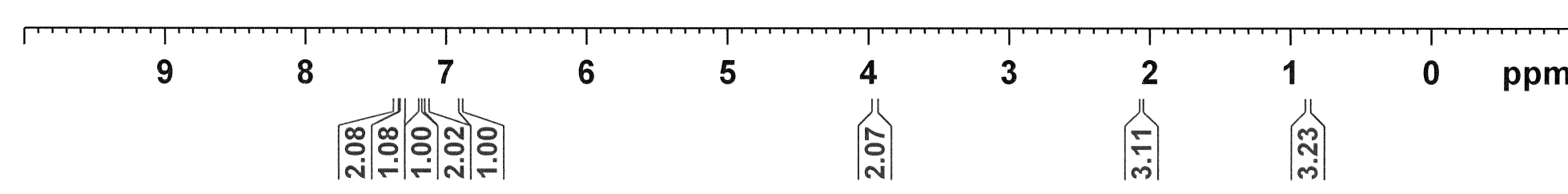




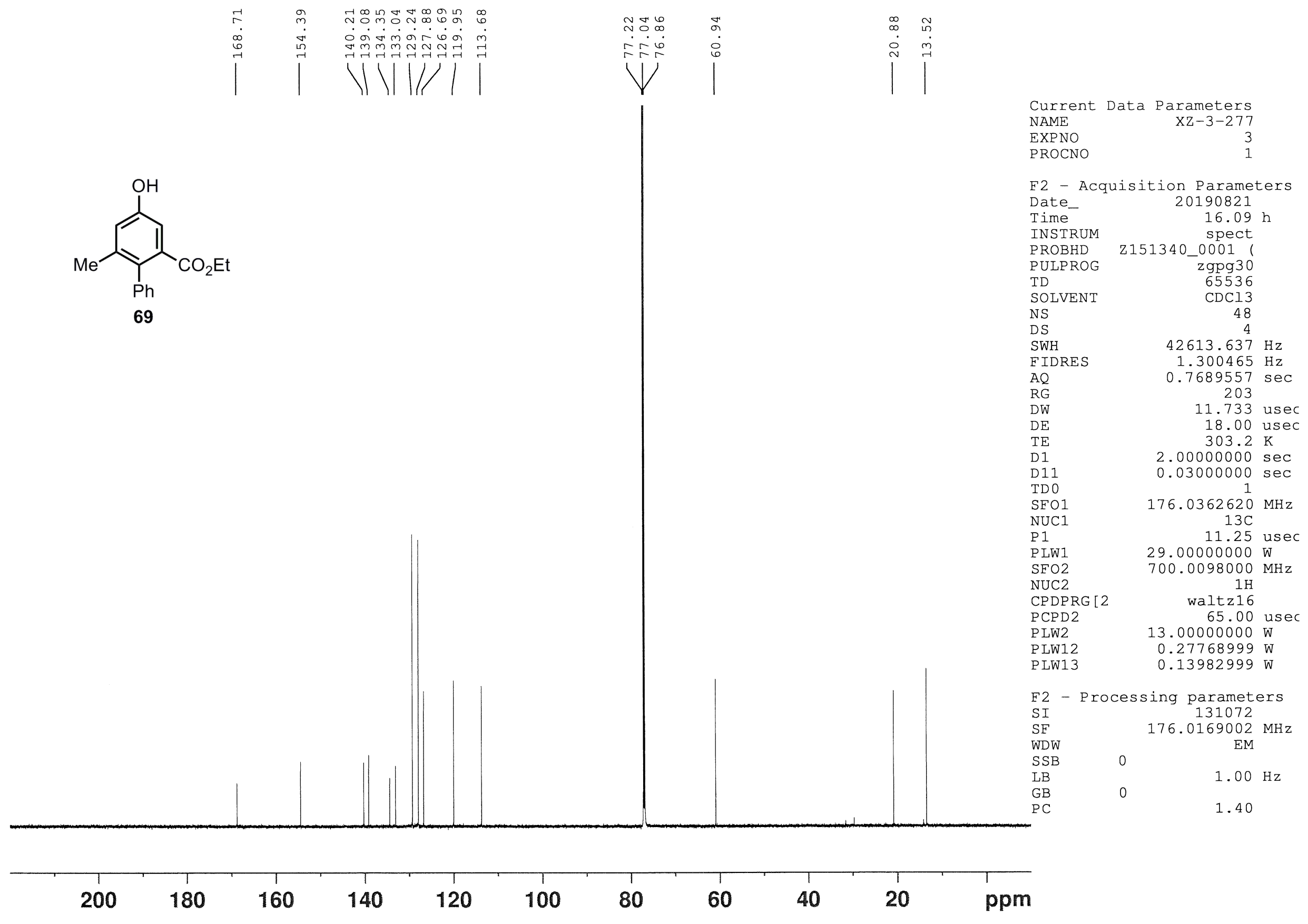




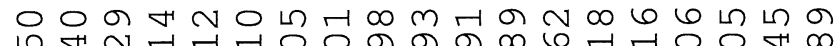
m m m

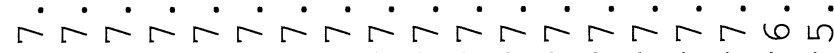

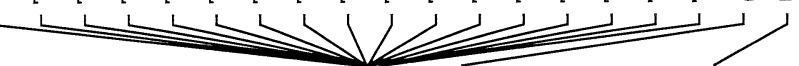

เกเกเก เก बু

$\dot{m} \dot{m} \dot{m}$

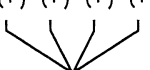

の ब m N $\sim \dot{N} \sim \dot{0} 00000$

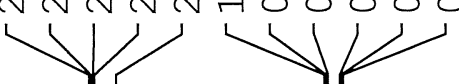<smiles>CCOC(=O)c1c(CC)c(O)cc(CC)c1P(O)O</smiles>

F2 - Acquisition Parameter Date__ 20181203 INSTRUM $17.40 \mathrm{~h}$ PROBHD Z151340_0001 ( PULPROG zg30 TD 65536 SOLVENT CDCl3 $\begin{array}{lr}\text { NS } & 16\end{array}$ SWH FIDRES $\mathrm{AQ}$ RG

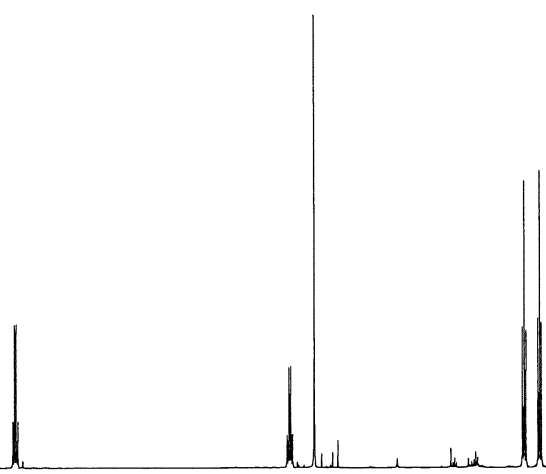

P 1 P LW1

$10504.202 \mathrm{~Hz}$ $0.320563 \mathrm{~Hz}$ $3.1195135 \mathrm{sec}$ 5.6

47.600 usec 10.00 usec $298.2 \mathrm{~K}$

$1.00000000 \mathrm{sec}$ $700.0115500 \mathrm{MHz}$ $1 \mathrm{H}$
9.50 usec SI Processing parameter 700.0070154

$S S B$

B

GB

PC

${ }_{0}^{0} \mathrm{~Hz}$

no

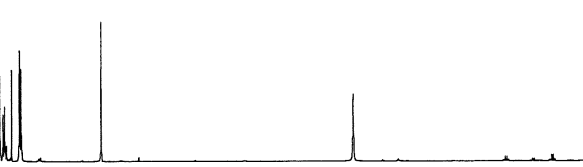

1.00

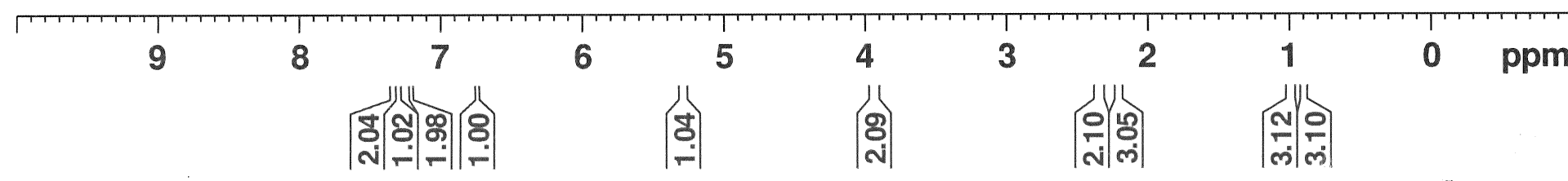



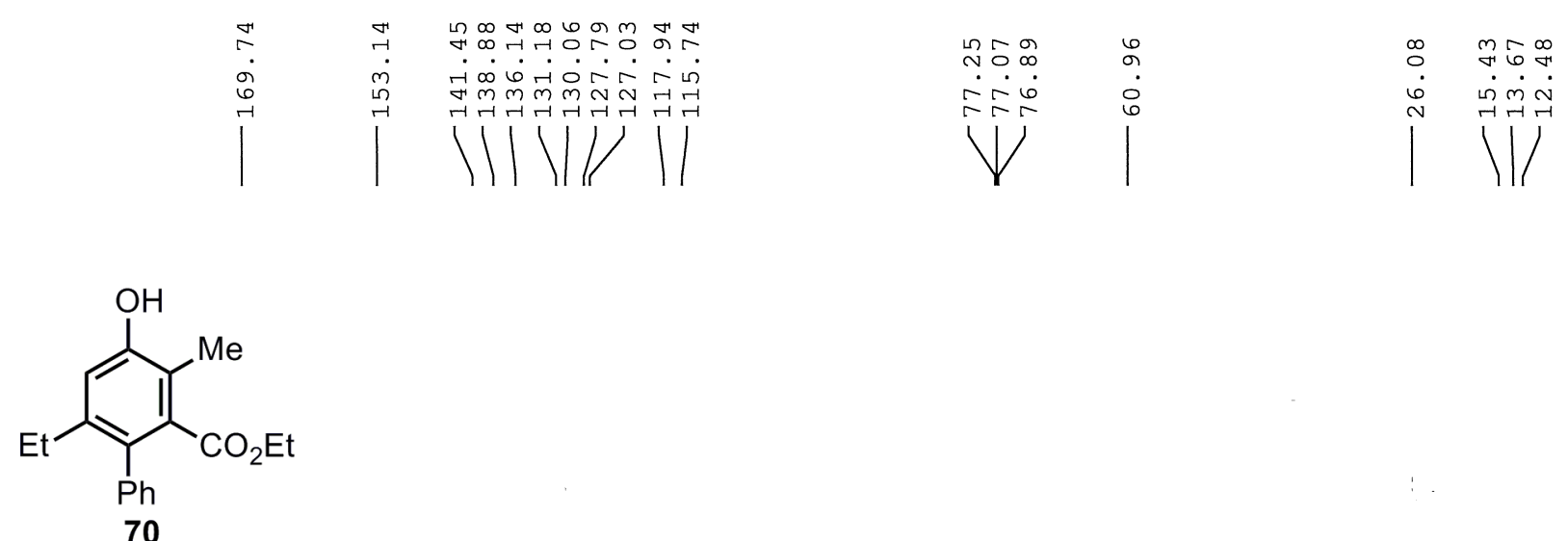

Current Data Parameters

EXPNO

arameter

PROCNO

F2 - Acquisition Parameter Date_ 20181203

Time

INSTRUM

PROBHD

PULPROG

17.46

TD

SOIVENT

NS

DS

FIDRES

FID
AQ
RG

RG

$\mathrm{DE}$

TE

D 1

D 11

SF O1

$\mathrm{P} 1$

$\mathrm{PLW} 1$

$\mathrm{SFO} 2$

$\mathrm{NUC} 2$

CPDPRG [ 2

PCPD 2

P LW2

P LW1 2

P LW1 3

z151340 spect

- 0001

$\mathrm{zgpg} 30$
65536

$\mathrm{CDC} 13$

25
4

$42613.637 \mathrm{~Hz}$

$1.300465 \mathrm{~Hz}$ $0.7689557 \mathrm{sec}$ 203

11.733 usec 18.00 usec $298.2 \mathrm{~K}$

$2.00000000 \mathrm{sec}$ $0.03000000 \mathrm{sec}$

$176.0362620 \mathrm{MHz}$

11.25 usec

$29.00000000 \mathrm{~W}$

$700.0098000 \mathrm{MHz}$

$$
\begin{gathered}
1 \mathrm{H} \\
\text { waltz } 16
\end{gathered}
$$$$
65.00 \text { use }
$$

$13.00000000 \mathrm{~W}$

$0.27768999 \mathrm{~W}$

$0.13982999 \mathrm{~W}$

F2 - Processing parameters

SI Processing paramet

SF

WDW

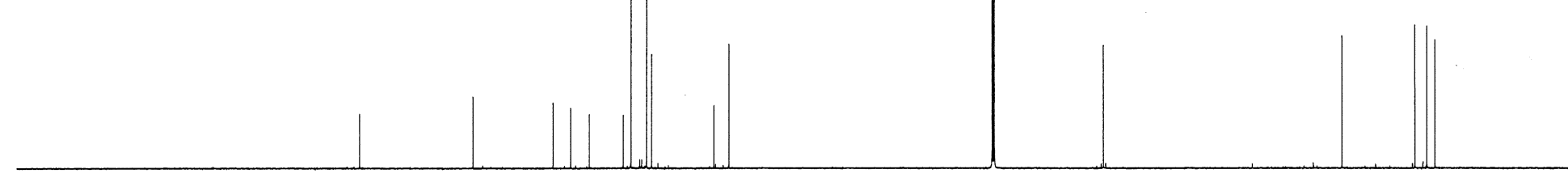

SSB

$176.0169002 \mathrm{MHz}$ $\mathrm{EM}$

$1.00 \mathrm{~Hz}$

1.40

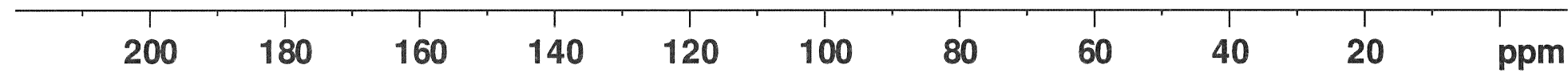


LI H R O Am NoO $m m m m m \sim N \sim \sim$

arrararin
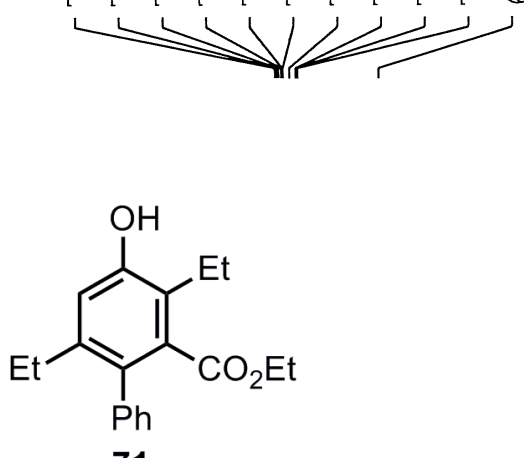

$\infty$ म m

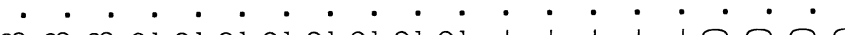

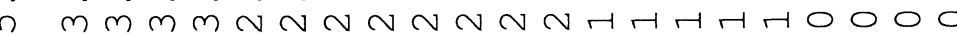

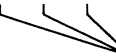

Current Data Parameters

NAME

EXPNO

PROCNO

F2 - Acquisition Parameters Date_ 20190430

Time $\quad 10.16$

INSTRUM spect

PROBHD Z151340_0001 (

PULPROG $\quad$ zg30

Zg30
PULPROG

SOLVENT

NS

DS

FIDRES

$A Q$
$R G$
RW

DW

DE

TE

TD 0

$\mathrm{SFO} 1$

NUC

P1

PLW1

SI 131072

SF

SSB

SSB

LB

PC

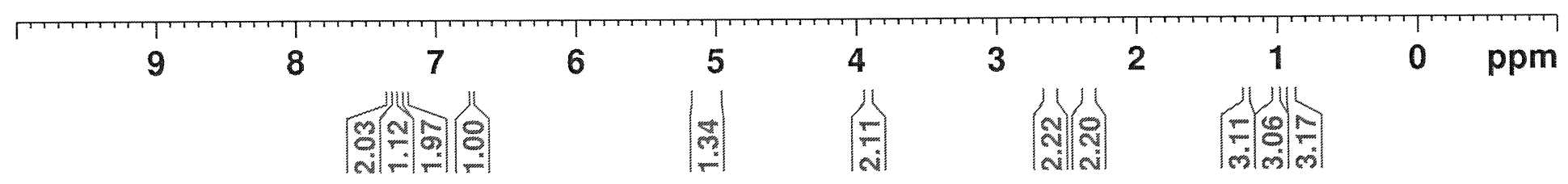




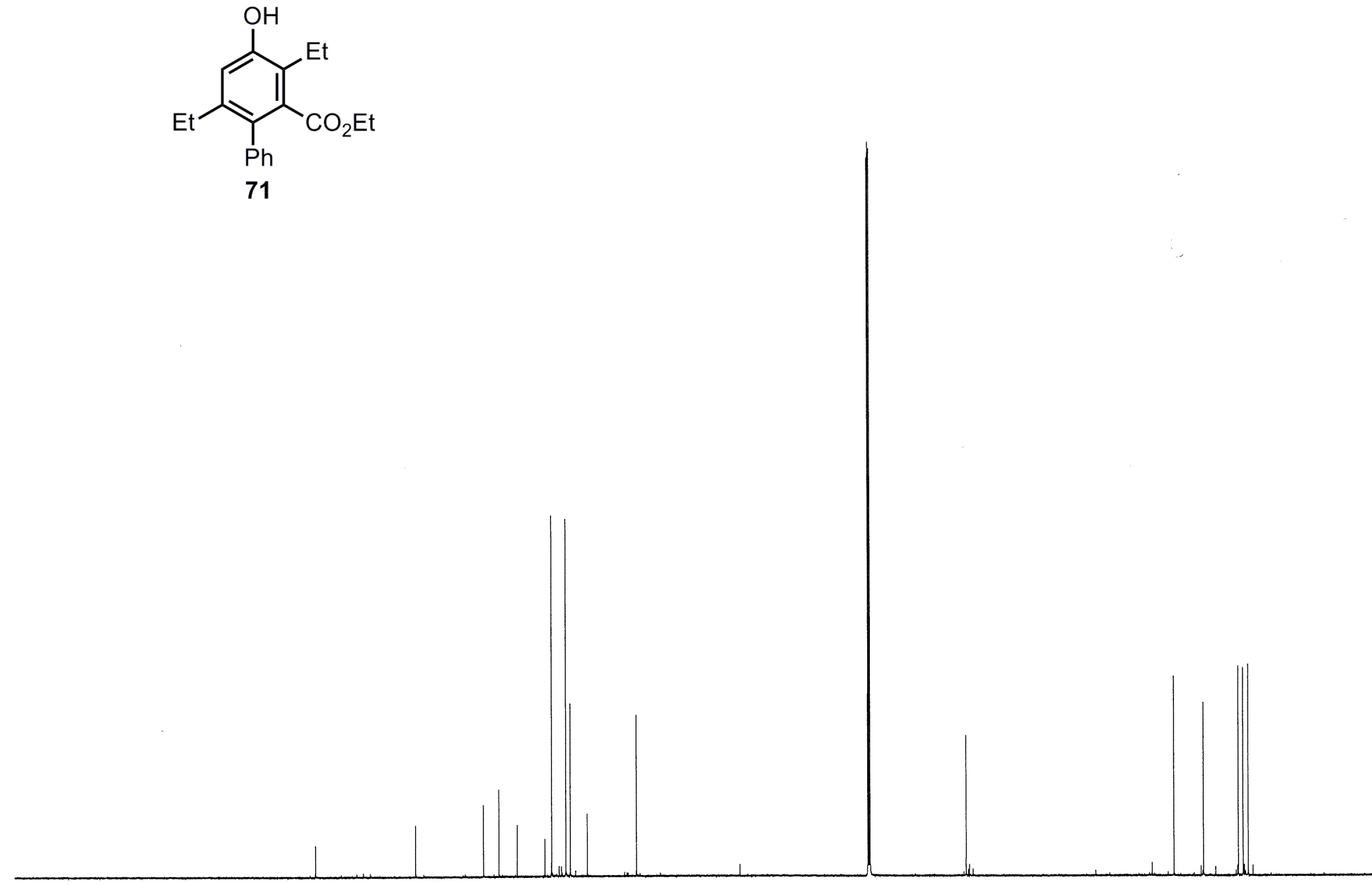

Parameters ROCNO $\mathrm{Xz}-3-137$

2 - Acquisition Parameter

Date_ 20190422

Time

$15.22 \mathrm{~h}$

INSTRUM

Z151340_0001

Z z gP 30

65536

SOLVENT CDCl3

DS

SWH

EIDRES

$\mathrm{AQ}$

RG

$D W$

DE

D 1
D 11
TD 0

TDO

$\mathrm{SFO1}$

(1)

P 1

P LW1

$\mathrm{SFO} 2$

UUC2

CPDPRG [ 2

P CP D 2

P LW2

P LW1 2

51
4

$42613.637 \mathrm{~Hz}$

$1.300465 \mathrm{~Hz}$

$0.7689557 \mathrm{sec}$

1.733 usec

18.00 usec

$303.2 \mathrm{~K}$

$2.00000000 \mathrm{sec}$

$0.03000000 \mathrm{sec}$

1

176.0362620 135

29.0000 .25 use $29.00000000 \mathrm{~W}$
$700.0098000 \mathrm{MHz}$

PLW1 3

$$
\text { waltz } 16
$$
65.00 use

$13.00000000 \mathrm{~W}$

$0.27768999 \mathrm{~W}$

$0.13982999 \mathrm{~W}$

F2 - Processing parameters SI 131072 SF $\quad 176.0169002 \mathrm{MHz}$

WDW EM

$S S B$

$L B$

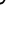

$1.00 \mathrm{~Hz}$

1.40

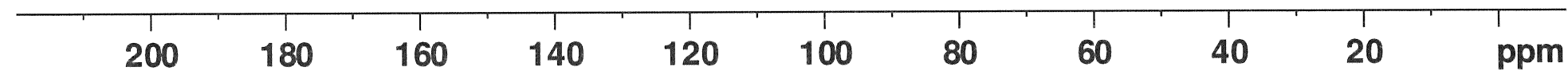


$\infty \infty \Gamma \circ \sigma \infty \pi)$ คิ - ir iririririo

ᄂ $<1$
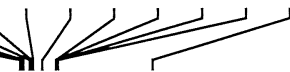
ம $m$ m m N N

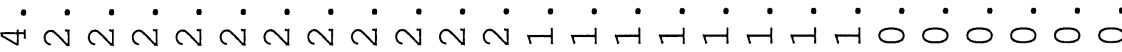

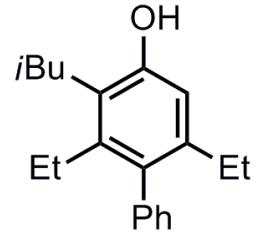

72

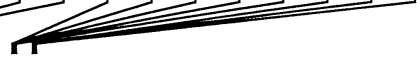

Current Data Parameters NAME

EXPNO

Parameters

PROCNO

1

F2 - Acquisition Parameter Date_ 20190519

Time $15.51 \mathrm{~h}$

INSTRUM

PROBHD

PULPROG

2151340 0001

$\begin{array}{ll}\text { TD } & 65536 \\ \text { SOLVENT } & \text { CDC13 }\end{array}$

DS

FIDRES

AQ

RG

$\mathrm{DE}$

$\mathrm{TE}$

D 1
TD 0

TDO

$\mathrm{SFO1}$

N 1

P LWI

CDC13

16

$10504.202 \mathrm{~Hz}$ $0.320563 \mathrm{~Hz}$ $3.1195135 \mathrm{sec}$ 5.6

47.600 usec 10.00 usec $303.2 \mathrm{~K}$

$1.00000000 \mathrm{sec}$

$700.0115500 \mathrm{MHz}$ $1 \mathrm{H}$

9.50 usec

$13.00000000 \mathrm{~W}$

F2 - Processing parameters SI 131072

SF $\quad 700.0070146 \mathrm{MHz}$

WDW

$\begin{array}{lll}\mathrm{SSB} & 0 & \\ \mathrm{LB} & 0 & \mathrm{~Hz}\end{array}$

1.00

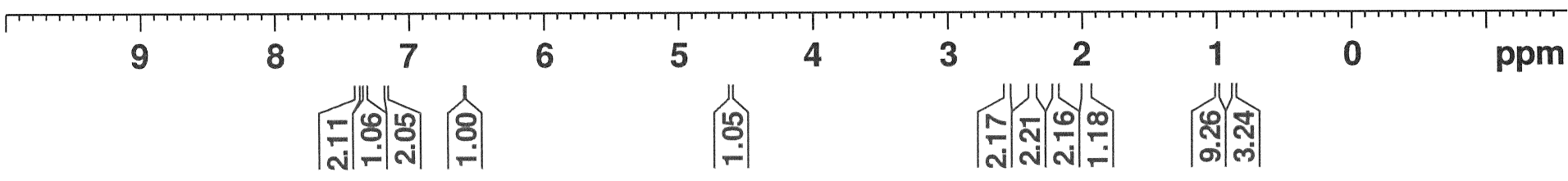




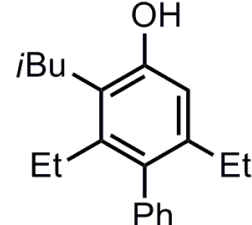

72
Current Data Parameters NAME

EXPNO

PROCNO

F2 - Acquisition Parameters

Date_ 20190519

Time $\quad 16.04 \mathrm{~h}$

INSTRUM spect

PROBHD Z151340_0001

PULPROG Z $\quad$ zgpg30

TD 65536

SOLVENT CDCl3

sol

DS

SWH

FIDRES

AQ

DW

$\mathrm{TE}$

D 1

D 11

SFO1

SFO1

$\mathrm{P} 1$

PLW1

$\mathrm{SFO} 2$

NUC2

CPDPRG [2

PCPD2

PLW2

PLW12

P LW13

F2 - Processing parameters

SI Processing paramete 131072

$\begin{array}{lc}\text { SF } & 176.0169002 \\ \text { WDW } & \text { MM }\end{array}$

WDW

SSB

LB

$\mathrm{GB}$
$\mathrm{PC}$

$42613.637 \mathrm{~Hz}$

$1.300465 \mathrm{~Hz}$

$0.7689557 \mathrm{sec}$

11.733 usec 18.00 usec

2.00000000

$0.03000000 \mathrm{sec}$

$176.0362620 \mathrm{MHz}$ $13 \mathrm{C}$

$29.00000000 \mathrm{~W}$ $700.0098000 \mathrm{MHz}$

waltzi6

65.00 usec

$13.00000000 \mathrm{~W}$

0.27768999

$1.00 \mathrm{~Hz}$

1.40

\begin{tabular}{|c|c|c|c|c|c|c|c|c|c|c|}
\hline 200 & 180 & 160 & 140 & 120 & 100 & 80 & $\begin{array}{c}1 \\
60\end{array}$ & 40 & $\begin{array}{c}1 \\
20\end{array}$ & ppm \\
\hline
\end{tabular}


mmN m $m$ m $m$ m

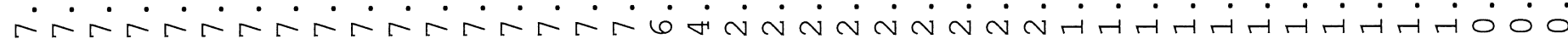
r<smiles>CCc1cc(-c2ccccc2)c(CC)c(CC(C)C)c1O</smiles>

73

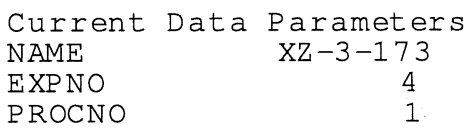

F2 - Acquisition Parameter Date__ 20190521

Time

$10.39 \mathrm{~h}$

INSTRUM

PROBHD

PULPROG spect

TD

SOLVENT

NS

DS

FIDRES

AO

RG

DW

$\mathrm{DE}$

TI

TD 0

$\mathrm{SFO} 1$

NUC1

$\mathrm{P} 1$

PLW1 Z151340_0001

Z

CDC 13

16

$10504.202 \mathrm{~Hz}$ $0.320563 \mathrm{~Hz}$ $3.1195135 \mathrm{sec}$ 6.35

47.600 usec 10.00 usec $303.2 \mathrm{~K}$

$1.00000000 \mathrm{sec}$ $700.0115500 \mathrm{MHz}$ $1 \mathrm{H}$

$13.00000000 \mathrm{~W}$

2 - Processing parameters SI 131072

SF $\quad 700.0070164 \mathrm{MHz}$

WDW

SSB

$0 \mathrm{~Hz}$

$0 \mathrm{~Hz}$

no

GB

1.00

PC
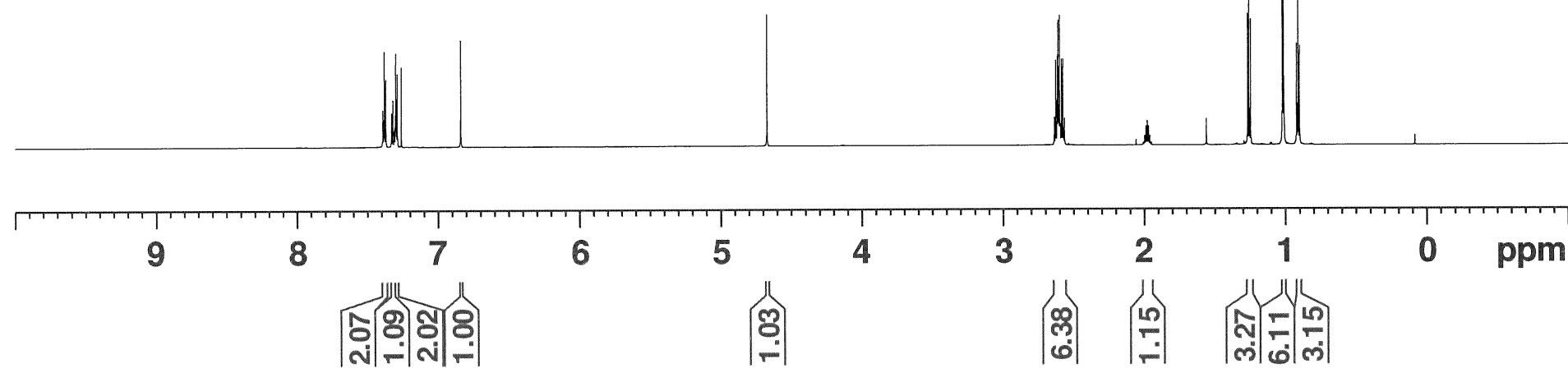


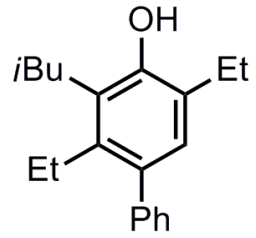

73

Current Data Parameters

NAME

EXPNO

PROCNO

F2 - Acquisition Parameters

Date_ 20190521

Time $\quad 10.53 \mathrm{~h}$

INSTRUM spect

PROBHD Z151340 0001 (

PULPROG Zgpg30

TD 65536

SOLVENT

NS

DS

SWH

FIDRES

AQ

RG

$\mathrm{DE}$

TE

D11

TDO

$\mathrm{SFO} 1$

NUC1

P1

PLW1

$\mathrm{SFO} 2$

NUC2

CPDPRG [ 2

PCPD 2

PLW2

PLW12

PLW13

CDCl3

47

$42613.637 \mathrm{~Hz}$

$1.300465 \mathrm{~Hz}$

$0.7689557 \mathrm{sec}$$$
203
$$

11.733 usec

18.00 usec $303.2 \mathrm{~K}$

$2.00000000 \mathrm{sec}$ $0.03000000 \mathrm{sec}$

$0.03000000 \mathrm{sec}$

$176.0362620 \mathrm{MHz}$ $13 \mathrm{C}$

11.25 usec $29.00000000 \mathrm{~W}$ $700.0098000 \mathrm{MHz}$

$1 \mathrm{H}$

65.00 usec

$13.00000000 \mathrm{~W}$

$0.27768999 \mathrm{~W}$

$0.27768999 \mathrm{~W}$

F2 - Processing parameters

SI 131072

SF $\quad 176.0169002 \mathrm{MHz}$

WDW

SSB

$\mathrm{LB}$

GB

$1.00 \mathrm{~Hz}$

1.40

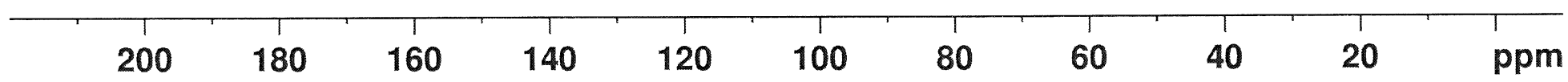


으유 क

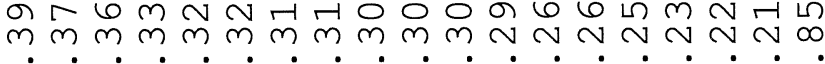

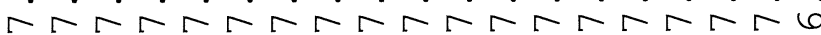
$\longrightarrow$

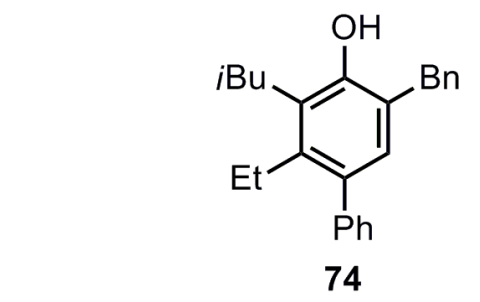

74

11.
न

6.

$\rightarrow \quad \dot{\sim} \dot{\sim} \dot{\sim} \dot{\sim}$

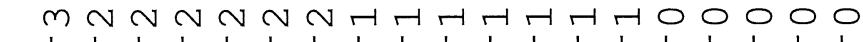

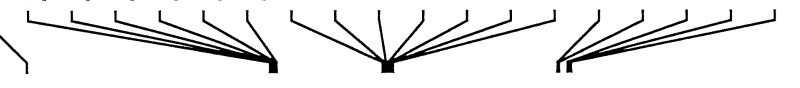
Current Data Parameters

F2 - Acquisition Parameter Date__ 20190729

Time

$12.15 \mathrm{~h}$

INSTRUM

PROBHD

PULPROG

spect

TD Z151340_0001

SOLVENT

NS

DS

EIDRES

AQ

RG

DW

DE

D1

TD 0

$\mathrm{SFO1}$

NUC1

$\mathrm{P} 1$

P LW1

$\mathrm{zg} 30$
65536

CDC 13

16

$10504.202 \mathrm{~Hz}$

$0.320563 \mathrm{~Hz}$

$3.1195135 \mathrm{sec}$ 22.6

47.600 usec 20.00 usec $303.4 \mathrm{~K}$

$1.00000000 \mathrm{sec}$ $700.0115500 \mathrm{MHz}$ $1 \mathrm{H}$ $1 \mathrm{H}$

$13.00000000 \mathrm{~W}$

2 - Processing parameters SI $\quad 131072$
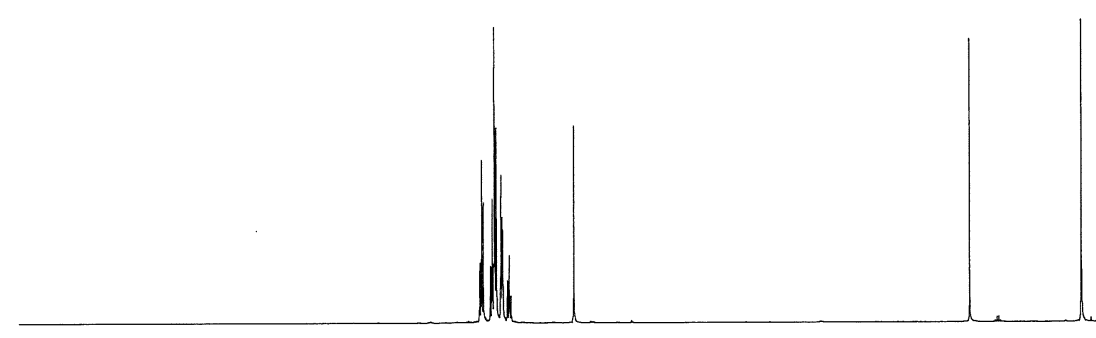

$700.0070166 \mathrm{MHz}$

SSB

no

0
0

GB

$\mathrm{H} \mathrm{Hz}$

1.00

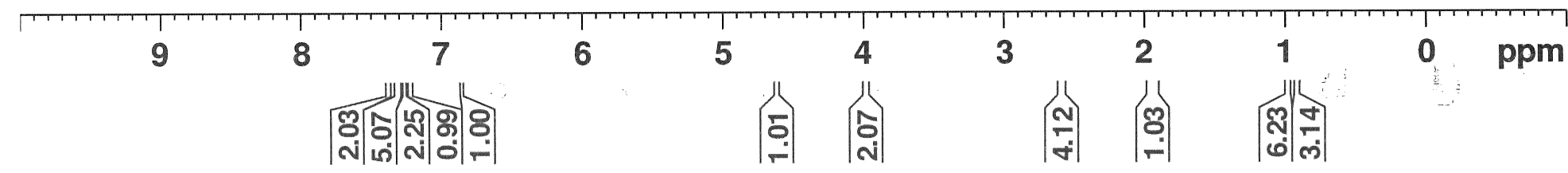


<smiles>CCc1c(-c2ccccc2)cc(Br)c(O)c1CC(C)C</smiles>

74

$\begin{array}{lr}\text { Current Data Parameters } \\ \text { NAME } & \text { XZ-3-256 }\end{array}$

EXPNO

PROCNO

F2 - Acquisition Parameters Date_ 20190729

Time-

INSTRUM

PROBHD

PROBHD

TD

TD

NS

DS

SWH

FIDRES

$\mathrm{AQ}$

RG

DW

TE

D1

D11

TDO

SFO1

P 1

PLW1

$\mathrm{SFO} 2$

NUC2

CPDPRG [ 2

PCPD2

PIV2

PLW12

PLW13

$12.27 \mathrm{~h}$

spect

00011

zgpg30

CDC13

102

$42613.637 \mathrm{~Hz}$

$1.300465 \mathrm{~Hz}$

$0.7689557 \mathrm{sec}$

1.733 usec

18.00 usec $303.2 \mathrm{~K}$

2.00000000 sec

$0.03000000 \mathrm{sec}$

1.03000000

$176.0362620 \mathrm{MHz}$ $13 \mathrm{C}$

$29.00000000 \mathrm{~W}$ $700.0098000 \mathrm{MHz}$

$$
\begin{array}{r}
1 \mathrm{H} \\
\text { waltz } 16
\end{array}
$$

65.00 usec

$13.00000000 \mathrm{~W}$

$0.27768999 \mathrm{~W}$

$0.13982999 \mathrm{~W}$

2 - Processing parameters

SI 131072

$\mathrm{SF} \quad 176.0169002 \mathrm{MHz}$

WDW

$\begin{array}{lll}\text { SSB } & 0 \\ \text { LB } & \end{array}$

$1.00 \mathrm{~Hz}$

1.40

\begin{tabular}{|c|c|c|c|c|c|c|c|c|c|}
\hline nח & 1 & 16 & 140 & 120 & 100 & $\begin{array}{l}1 \\
80\end{array}$ & 60 & 40 & ppm \\
\hline
\end{tabular}


글

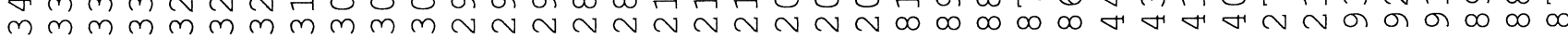

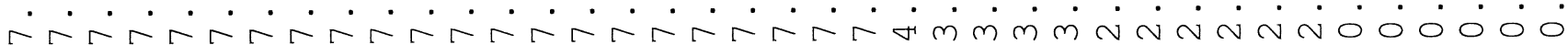
(l)<smiles>CCOC(=O)c1ccccc1[N+](=O)c1c(CC)c(C)c(C)c(O)c1OCC</smiles>

75

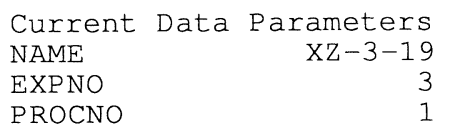

PROCNO

F2 - Acquisition Parameters Date__ 20190919

Time $\quad 10.17 \mathrm{~h}$

INSTRUM spect

PROBHD Z151340_0001 (

PULPROG zg30

TD 65536

SOLVENT CDCl3

NS

DS

SWH

FIDRES

AQ

RG

DW

$\mathrm{DE}$

$\mathrm{TE}$

D1

NUC1

PO

P 1

P 1

16

$10504.202 \mathrm{~Hz}$

$0.320563 \mathrm{~Hz}$

$3.1195135 \mathrm{sec}$ 32

47.600 usec

20.00 usec

$303.2 \mathrm{~K}$

$1.00000000 \mathrm{sec}$

$700.0115500 \mathrm{MHz}$

3. 17 usec 9.50 usec

$13.00000000 \mathrm{~W}$

F2 - Processing parameters SI 131072

SF $\quad 700.0070168 \mathrm{MHz}$

WDW

$\mathrm{SSB}$

$L B$

$G B$

no

0
0 $\mathrm{~Hz}$

0
0
0

1.00

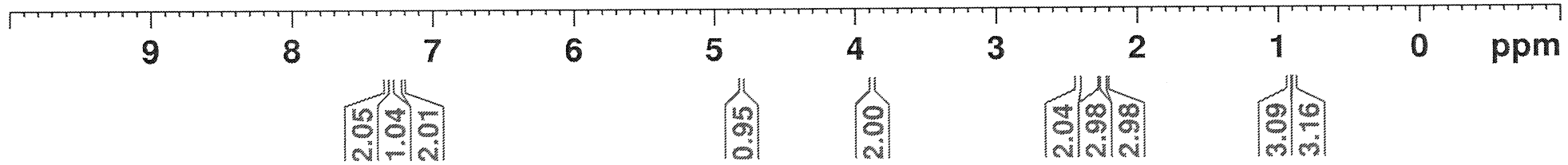




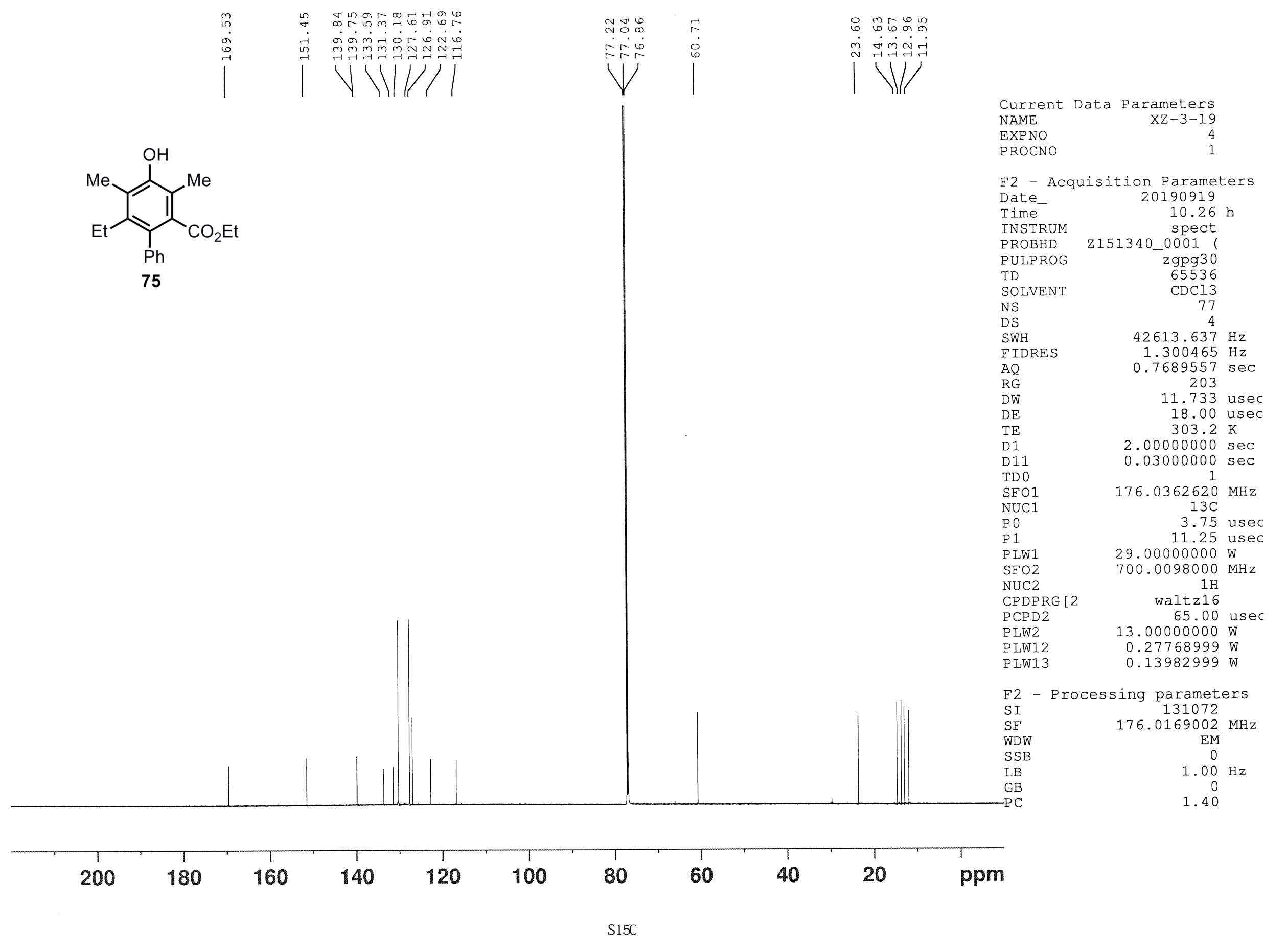


000 $m m m m \sim \sim \sim N$

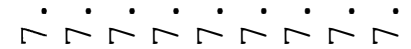
$\rightarrow$

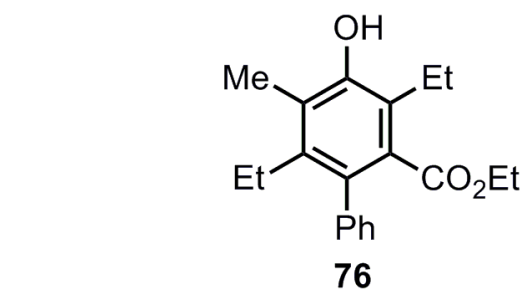

ののののतमㅇ の の

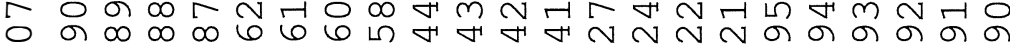
$\dot{m} \dot{m} \dot{m} \dot{m} \dot{N} \dot{N} \dot{N} \dot{N} \dot{N} \dot{N} \dot{r} \dot{r} \dot{0} \dot{0} \dot{0} \dot{0} \dot{0} \dot{0} \dot{0}$

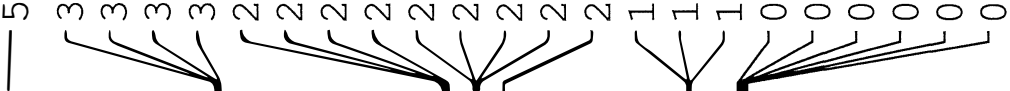

Current Data Parameter

$\mathrm{XZ}-3-222$

EXPNO

F2 - Acquisition Parameter Date__ 20190630

Time $16.05 \mathrm{~h}$

INSTRUM spect

PROBHD Z151340_0001

PULPROG zg30

$\begin{array}{lr}\text { TD } & 65536 \\ \text { SOLVENT } & \text { CDCl3 }\end{array}$

NS 16

SWH $10504.202 \mathrm{~Hz}$

FIDRES $\quad 0.320563 \mathrm{~Hz}$

$\mathrm{AQ} \quad 3.1195135 \mathrm{sec}$

RG

DW

DE

TE

TI

SFO1

SFO1

1

P 1

9
47.600 usec

20.00 usec

$303.2 \mathrm{~K}$

$1.00000000 \mathrm{sec}$

$700.0115500 \mathrm{MHz}$

9.50 usec

F2 - Processing parameters

SI $\quad 131072$

SF $\quad 700.0070151 \mathrm{MHz}$

no

SSB

LB

0

P C

1.00

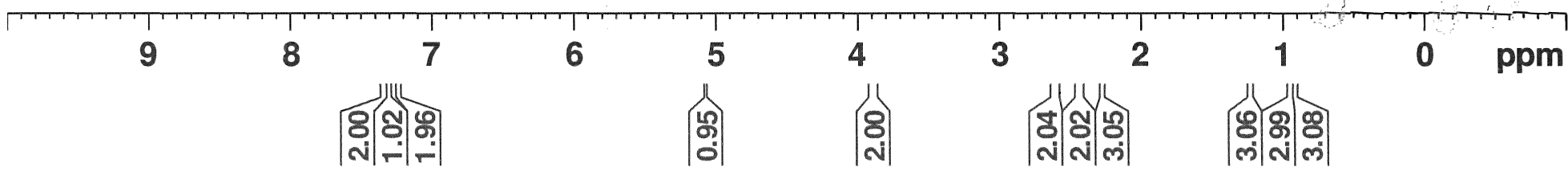




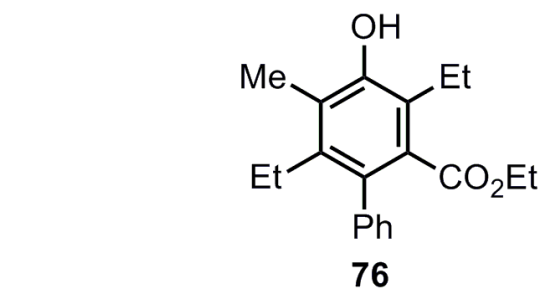

Current Data Parameters

F2 - Acquisition Parameter Date_ 20190630 Time $\quad 16.22 \mathrm{~h}$ INSTRUM spect PROBHD Z151340_0001 PULPROG zgpg30 TD 65536 SOLVENT CDC13 $\begin{array}{lc}\text { NS } & 92\end{array}$ SWH $\quad 42613.637 \mathrm{~Hz}$ FIDRES $\quad 1.300465 \mathrm{~Hz}$ $\mathrm{AQ}$ $\mathrm{RG}$ 18.00 usec $303.2 \mathrm{~K}$

$2.00000000 \mathrm{sec}$ $0.03000000 \mathrm{sec}$ $176.0362620 \mathrm{MHz}$ $13 \mathrm{C}$

29.00000000 use $29.00000000 \mathrm{~W}$ $700.0098000 \mathrm{MHz}$

PLW1 3 65.00 use $13.00000000 \mathrm{~W}$ $0.27768999 \mathrm{~W}$ $0.13982999 \mathrm{~W}$

F2 - Processing parameters SI 131072 SE $\quad 176.0169002 \mathrm{MHz}$ WDW SSB $L B$ $\mathrm{GB}$
$\mathrm{PC}$

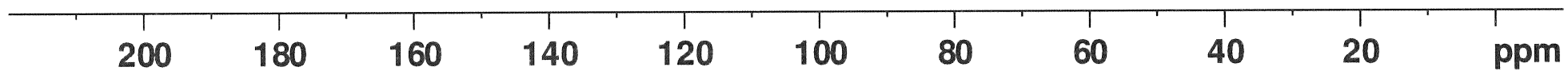


अ $m m m m \sim N N N$

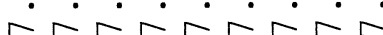
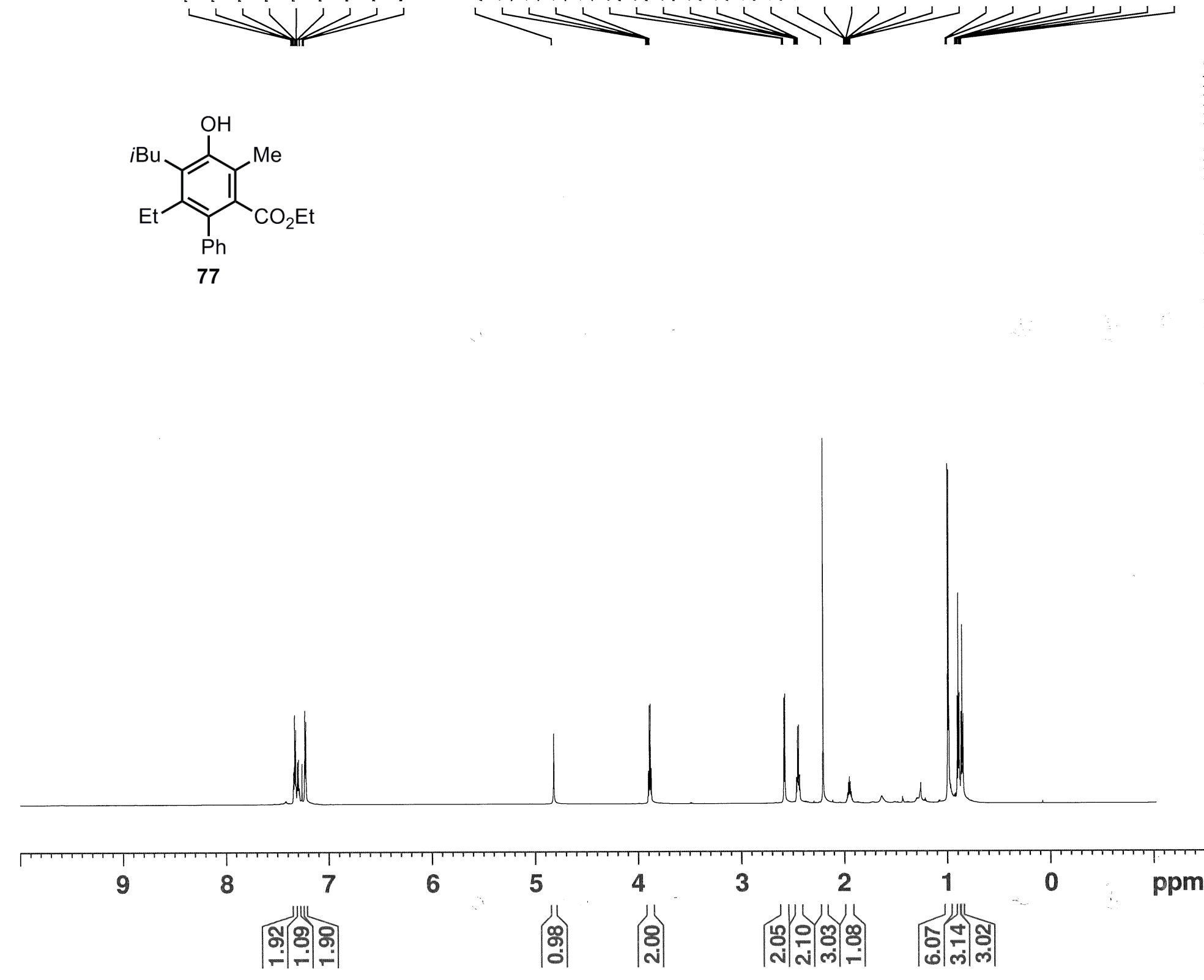

Current Data Parameters NAME XZ-3-35-3 XPNO 1

F2 - Acquisition Parameter Date__ 20181217 Time $12.16 \mathrm{~h}$ INSTRUM spect PROBHD Z151340_0001 PULPROG Z151340 2030 TD NS 16 DS FIDRES $\mathrm{AQ}$ RG

DW

$\mathrm{DE}$

TI

TD 0

$\mathrm{SFO1}$

NUC 1

P 1

$10504.202 \mathrm{~Hz}$ $0.320563 \mathrm{~Hz}$ $3.1195135 \mathrm{sec}$ 6.35

47.600 usec 10.00 usec $300.1 \mathrm{~K}$

$1.00000000 \mathrm{sec}$ $700.0115500 \mathrm{MHz}$ 9.50 usec $13.00000000 \mathrm{~W}$

- Processing parameters SI 131072

SF $\quad 700.0070158 \mathrm{MHz}$ $S$ B 0 $\mathrm{O} \mathrm{Hz}$ no OB C 

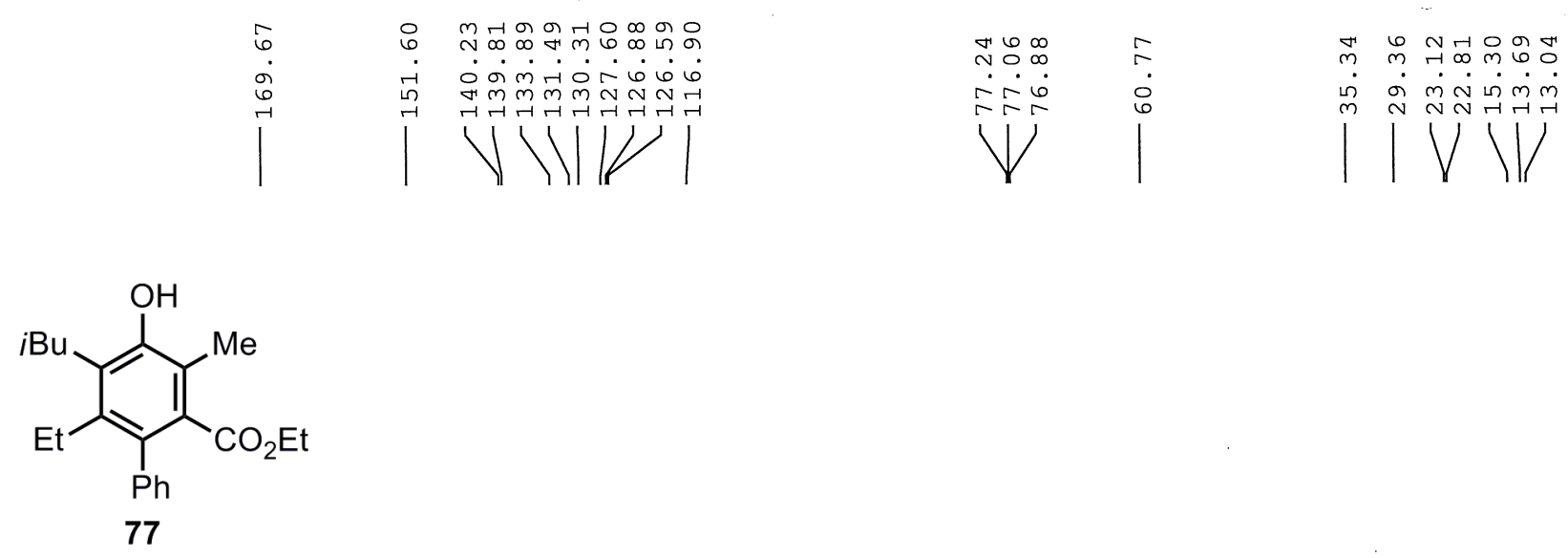
Current Data Parameters

F2 - Acquisition Parameter

Date_ 20181217

Time $\quad 12.26 \mathrm{~h}$

INSTRUM spect

PROBHD Z151340_0001

PULPROG zgpg30

TD 65536

SOLVENT CDCl3

NS

DS

FIDRES

FIDR
AQ
RG

DW

$D E$

TE

D 11

D 11

SFO1

$\mathrm{SFO1}$

$\mathrm{NUC} 1$
$\mathrm{P} 1$

P 1
P LW1

$\mathrm{SFO} 2$

NUC 2

CPDPRG [ 2

PCPD 2

PLW2

P LW1 2
P LW1 3 39

$42613.637 \mathrm{~Hz}$

$1.300465 \mathrm{~Hz}$ $0.7689557 \mathrm{sec}$ 203

11.733 usec 18.00 usec $300.0 \mathrm{~K}$

$2.00000000 \mathrm{sec}$ $0.03000000 \mathrm{sec}$

$176.0362620 \mathrm{MHz}$ $13 \mathrm{C}$

11.25 usec

$29.00000000 \mathrm{~W}$ $700.0098000 \mathrm{MH}$

$$
\text { waltz16 }
$$
65.00 use $13.00000000 \mathrm{~W}$ $0.27768999 \mathrm{~W}$ $0.13982999 \mathrm{~W}$

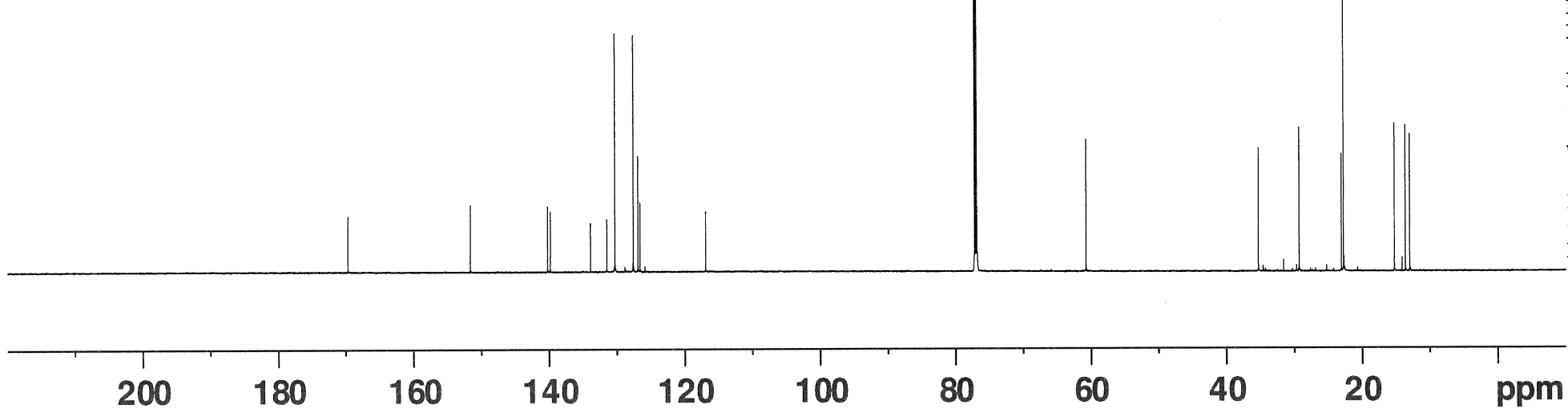

2 - Processing parameters

I 131072

SF $\quad 176.0169002 \mathrm{MHz}$

EMW

$\begin{array}{lll}S S B & 0 & 1.00 \mathrm{~Hz}\end{array}$

GB $\quad 0$

1.40 


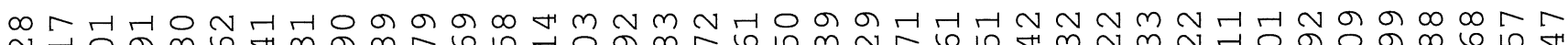
가 r r r
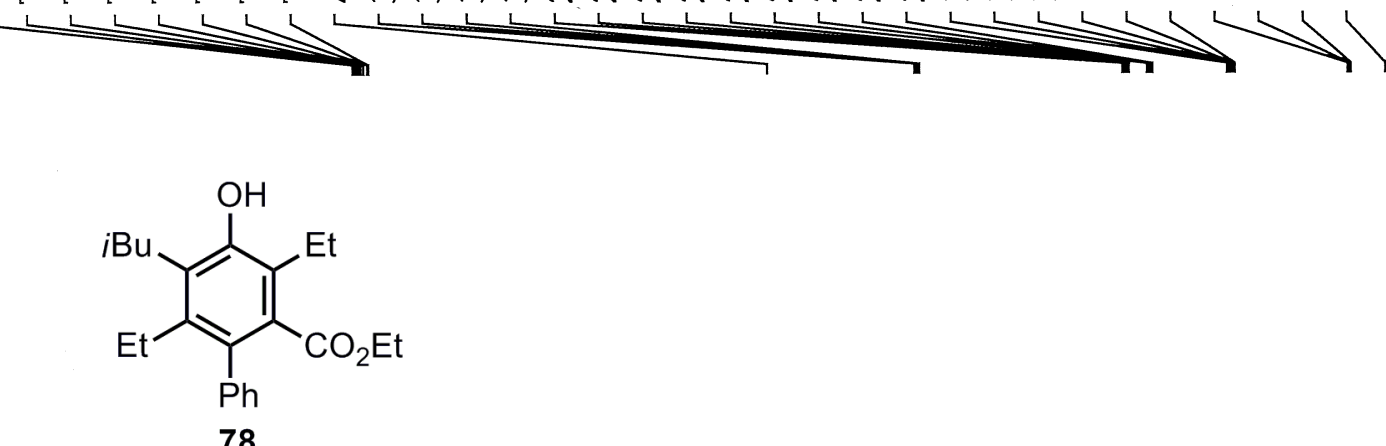

78

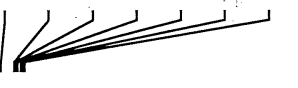

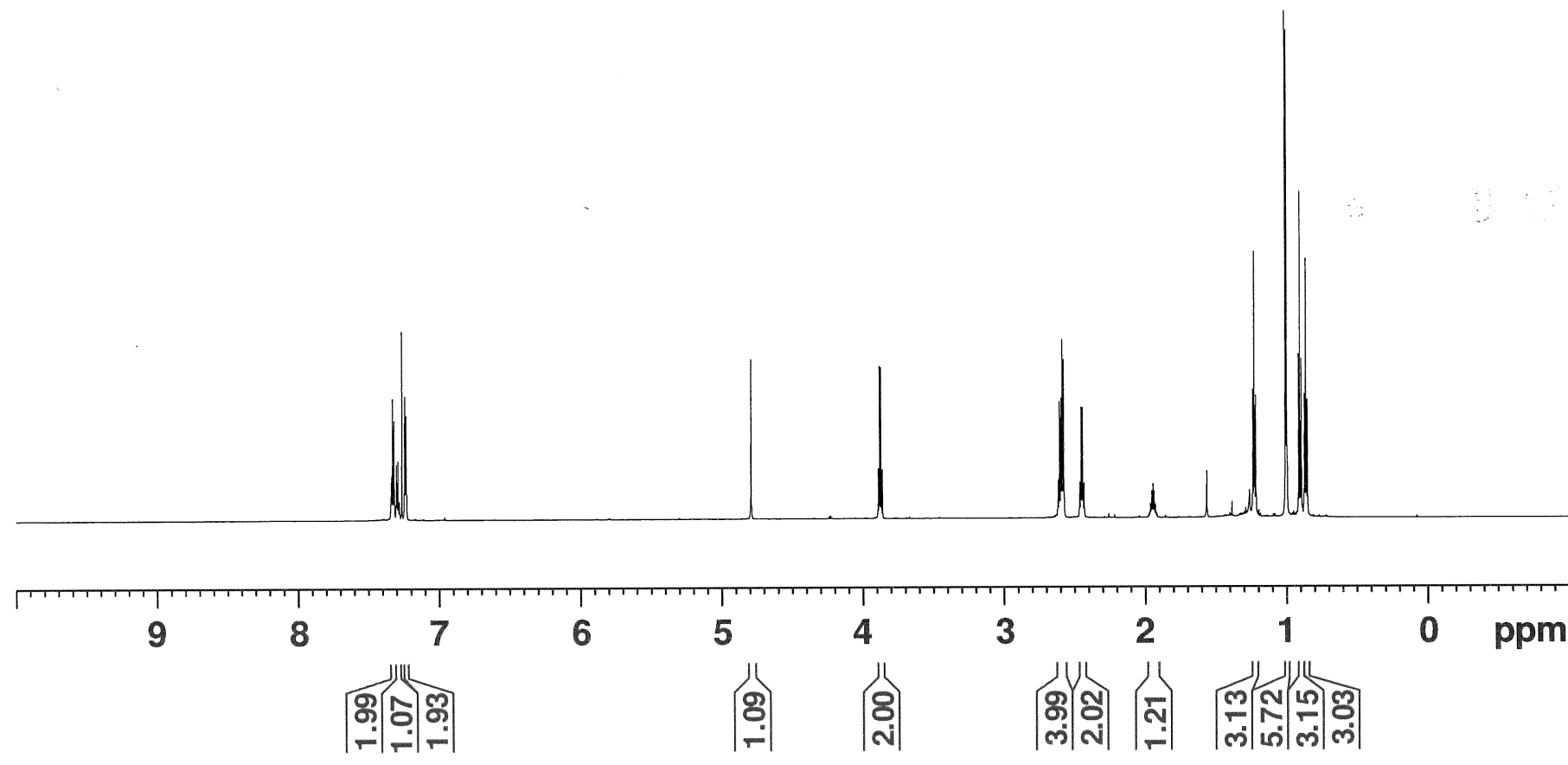




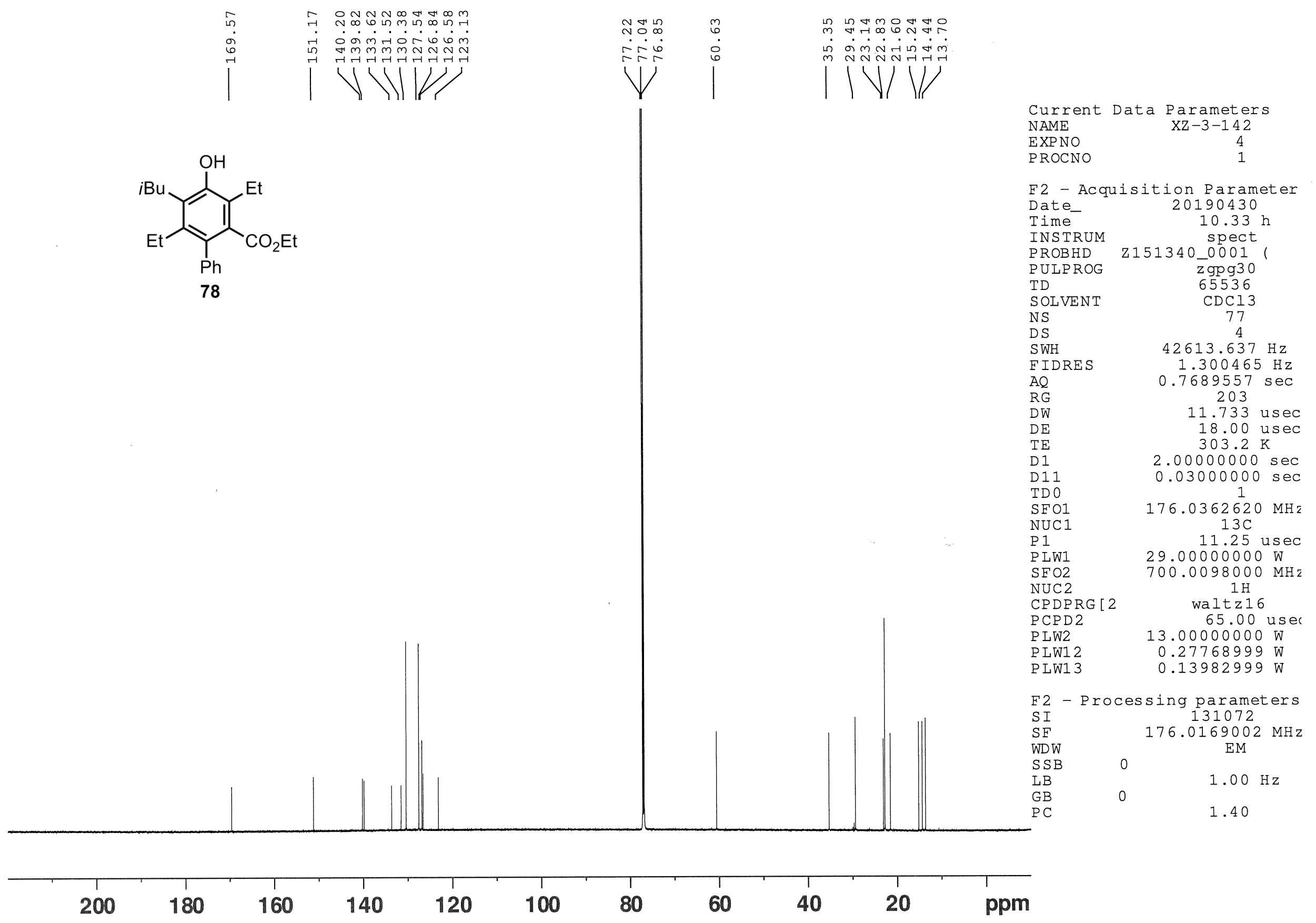


가유 m

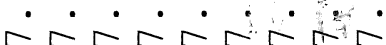
$\rightarrow$

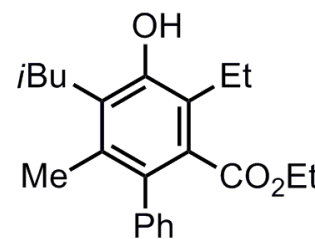

79 $m$ अ ๙ ब न ब

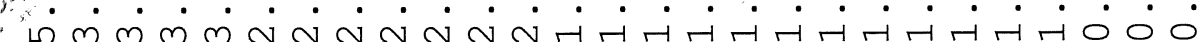
$\mathrm{S}^{\circ}$

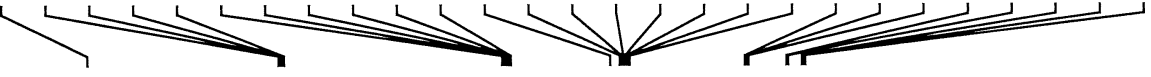

Current Data Parameters NAME

EXPNO PROCNO

$\mathrm{XZ}-3-246$

F2 - Acquisition Parameter

Date_ 20190721

Time

$15.15 \mathrm{~h}$

INSTRUM

PROBHD

PULPROG

$T D$

Z151340_0001

SOLVENT CDCl3

NS

16

DWH

FIDRES

$\mathrm{F} I \mathrm{IDR}$
$\mathrm{AQ}$

$\mathrm{AQ}$
$\mathrm{RG}$

DW

$\mathrm{DE}$

TE

TD 0

$\mathrm{SFO} 1$

NUC

P 1

$10504.202 \mathrm{~Hz}$

$0.320563 \mathrm{~Hz}$

$3.1195135 \mathrm{sec}$

.1195135
97.600

9

47.600 usec 20.00 usec $303.4 \mathrm{~K}$

$1.00000000 \mathrm{sec}$

$700.0115500 \mathrm{MHz}$

9. 50 usec

LW1

$13.00000000 \mathrm{~W}$

F2 - Processing parameters

$\begin{array}{lc}\text { SI } & 131072 \\ \text { SF } & 700.0070151 \mathrm{MHz}\end{array}$

WDW

SSB

LB

GB

0

$\mathrm{H} \mathrm{Hz}$

no

PC

1.00

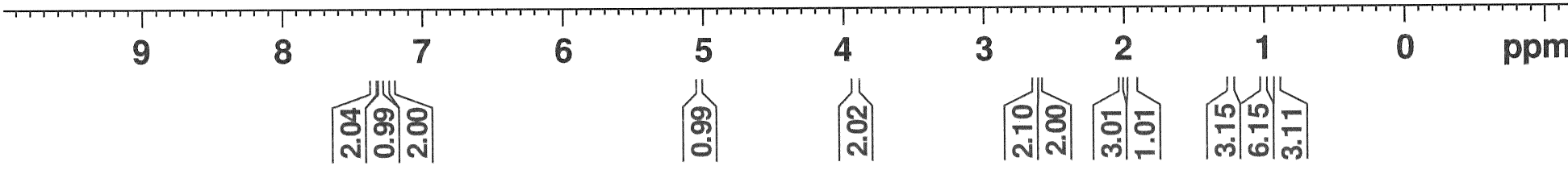



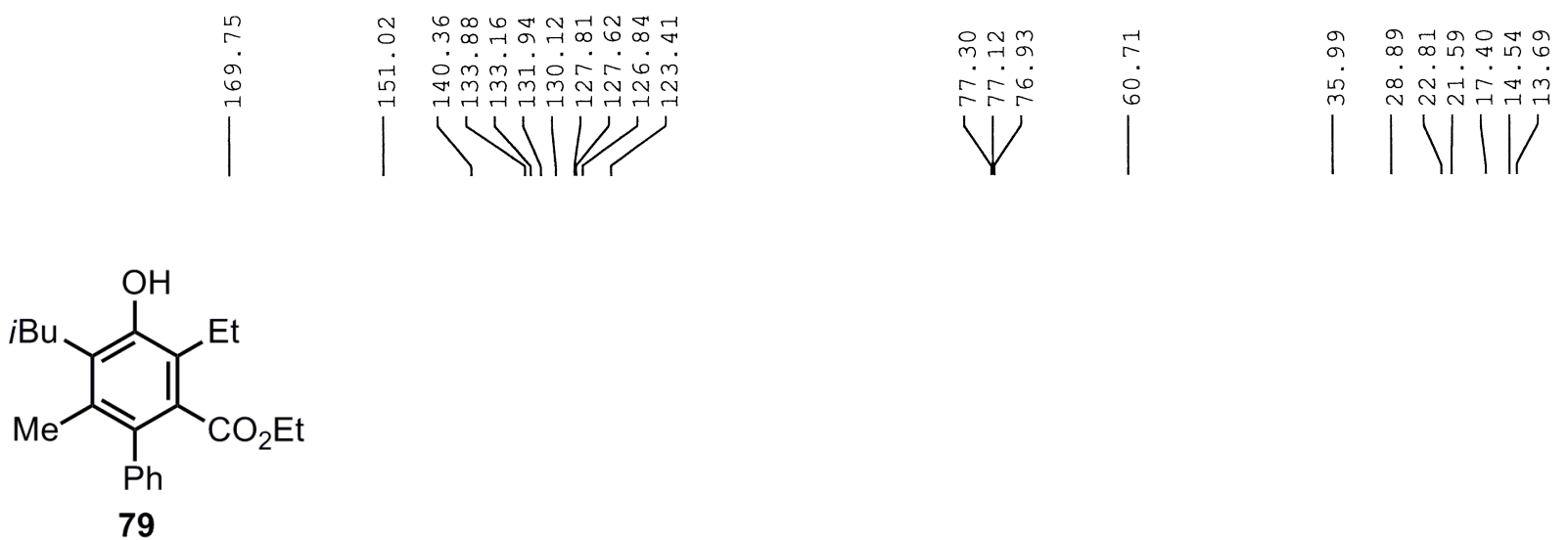

Current Data Parameters

NAME

EXPNO

PROCNO

$\mathrm{Xz}-3-246$

F2 - Acquisition Parameters

Date_ 20190721

Time $15.35 \mathrm{~h}$

INSTRUM spect

PROBHD Z151340_0001)

PULPROG zgpg30

$\begin{array}{ll}\text { TD } & 65536 \\ \text { SOLVENT } & \text { CDC13 }\end{array}$

NS

DS

SWH

$42613.637 \mathrm{~Hz}$

$1.300465 \mathrm{~Hz}$
H IDES

RG

RW

DE

TE

D1

D11

SFO1

SFO1

P 1

PLW1

$\mathrm{SFO} 2$

NUC2

CPDPRG [ 2

PCPD 2

PLW2

PLW12

$0.7689557 \mathrm{sec}$

203

11.733 usec

18.00 usec $303.2 \mathrm{~K}$

$2.00000000 \mathrm{sec}$ $0.03000000 \mathrm{sec}$ 1

$176.0362620 \mathrm{MHz}$ $13 \mathrm{C}$ $29.00000000 \mathrm{~W}$ $700.0098000 \mathrm{MHz}$

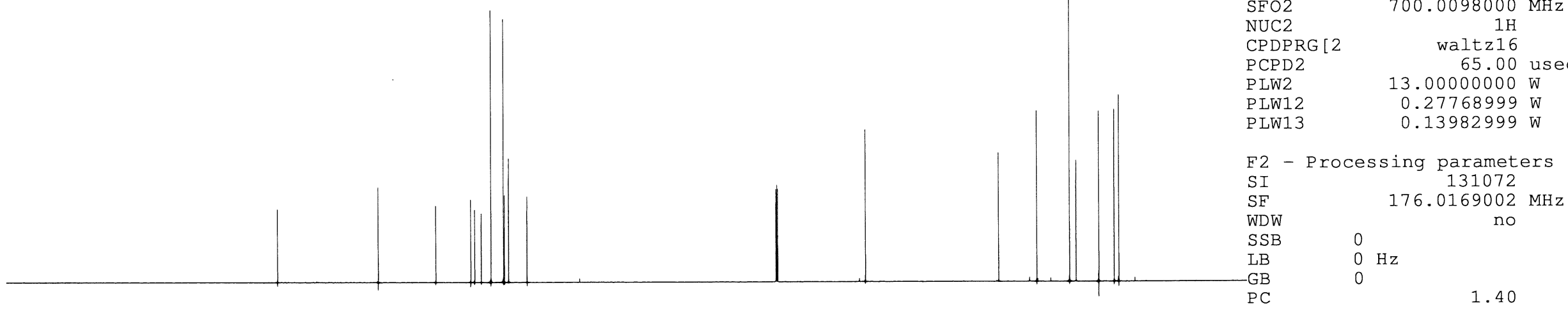


으

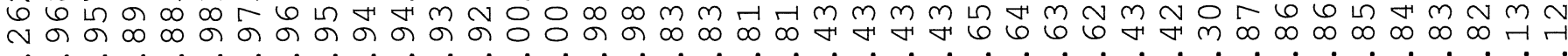

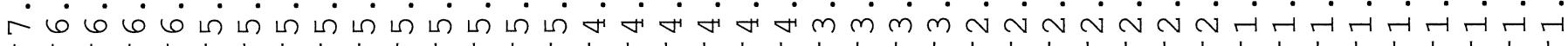

L

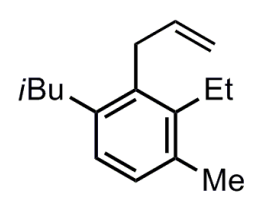

80

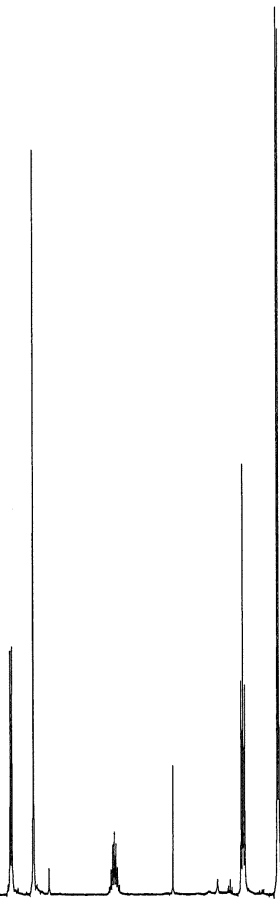

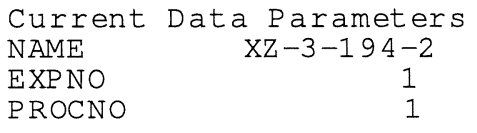

F2 - Acquisition Parameter Date_ 20190606 Time $\quad 14.21 \mathrm{~h}$ INSTRUM spect PROBHD Z566801_0015 PULPROG TD 65536 SOIVENT NS 16 SWH

FIDRES

$\mathrm{AQ}$

RG

DW

$\mathrm{DE}$

TI

TD 0

NEO1

P 1

PLW1

$10504.202 \mathrm{~Hz}$ $0.320563 \mathrm{~Hz}$ $3.1195135 \mathrm{sec}$ 203

47.600 usec 6.50 usec $303.2 \mathrm{~K}$

$1.00000000 \mathrm{sec}$ $700.0115500 \mathrm{MHz}$ $1 \mathrm{H}$
12.88 usec $40.00000000 \mathrm{~W}$

2 - Processing parameters SI $\quad 131072$

SF $\quad 700.0070166 \mathrm{MHz}$ WDW SSB $\quad 0$

$\mathrm{LB} \quad \mathrm{O} \mathrm{Hz}$

GB $\quad 0$ - 1.00

1.00
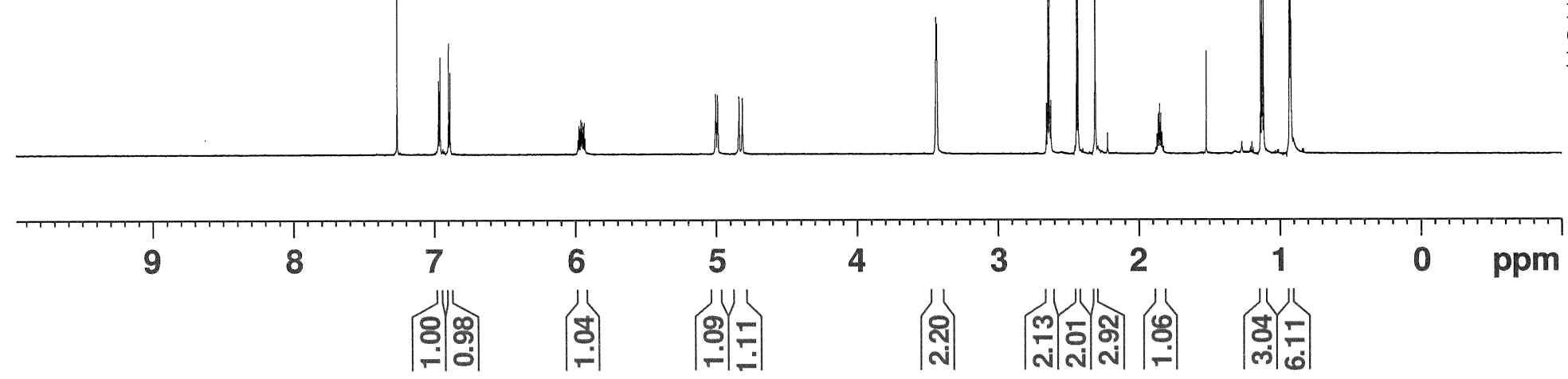


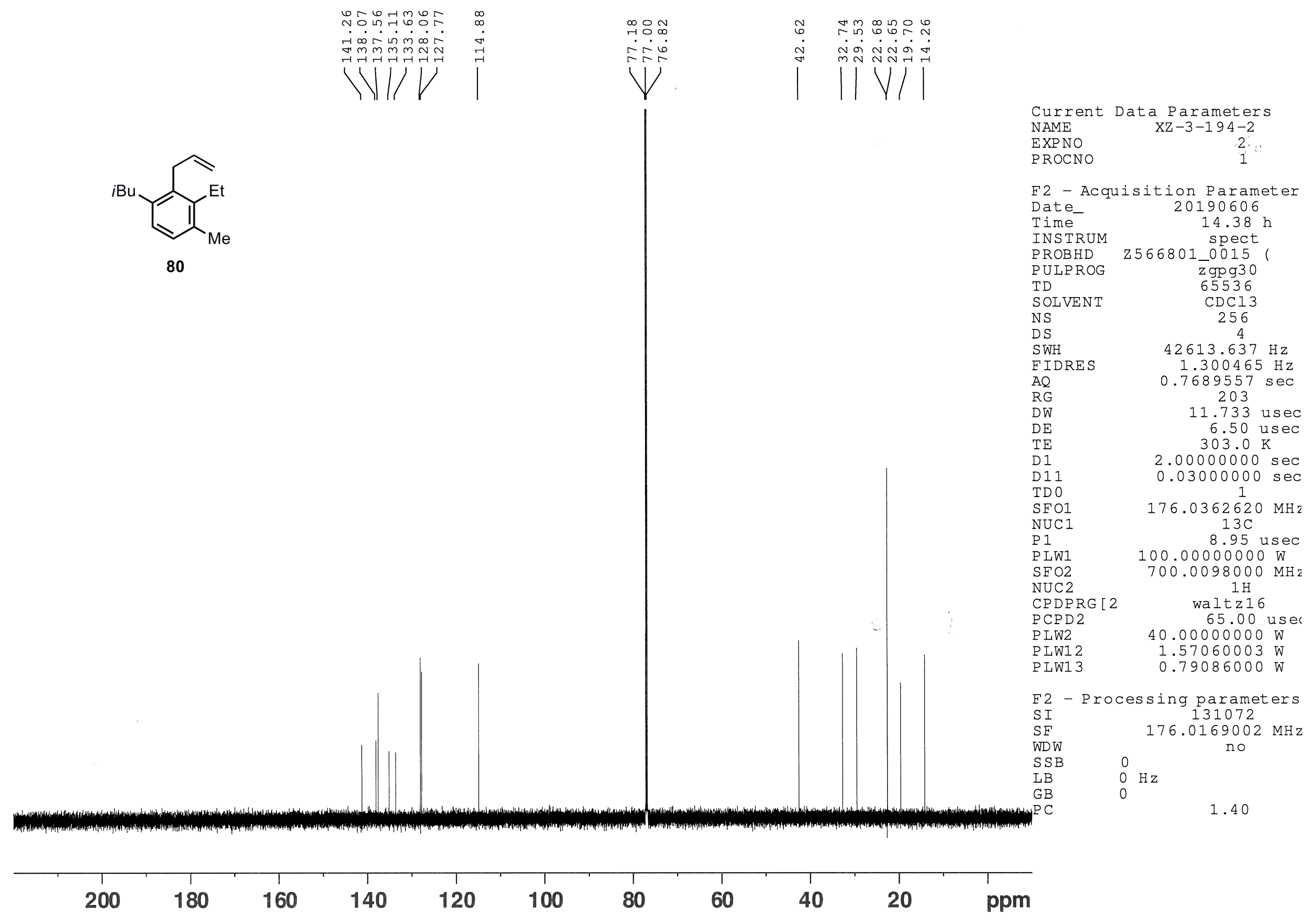




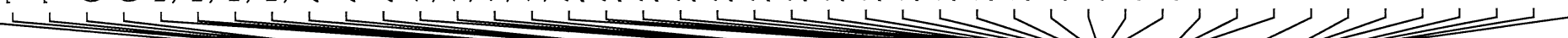

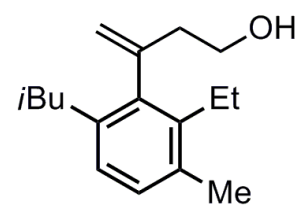

81
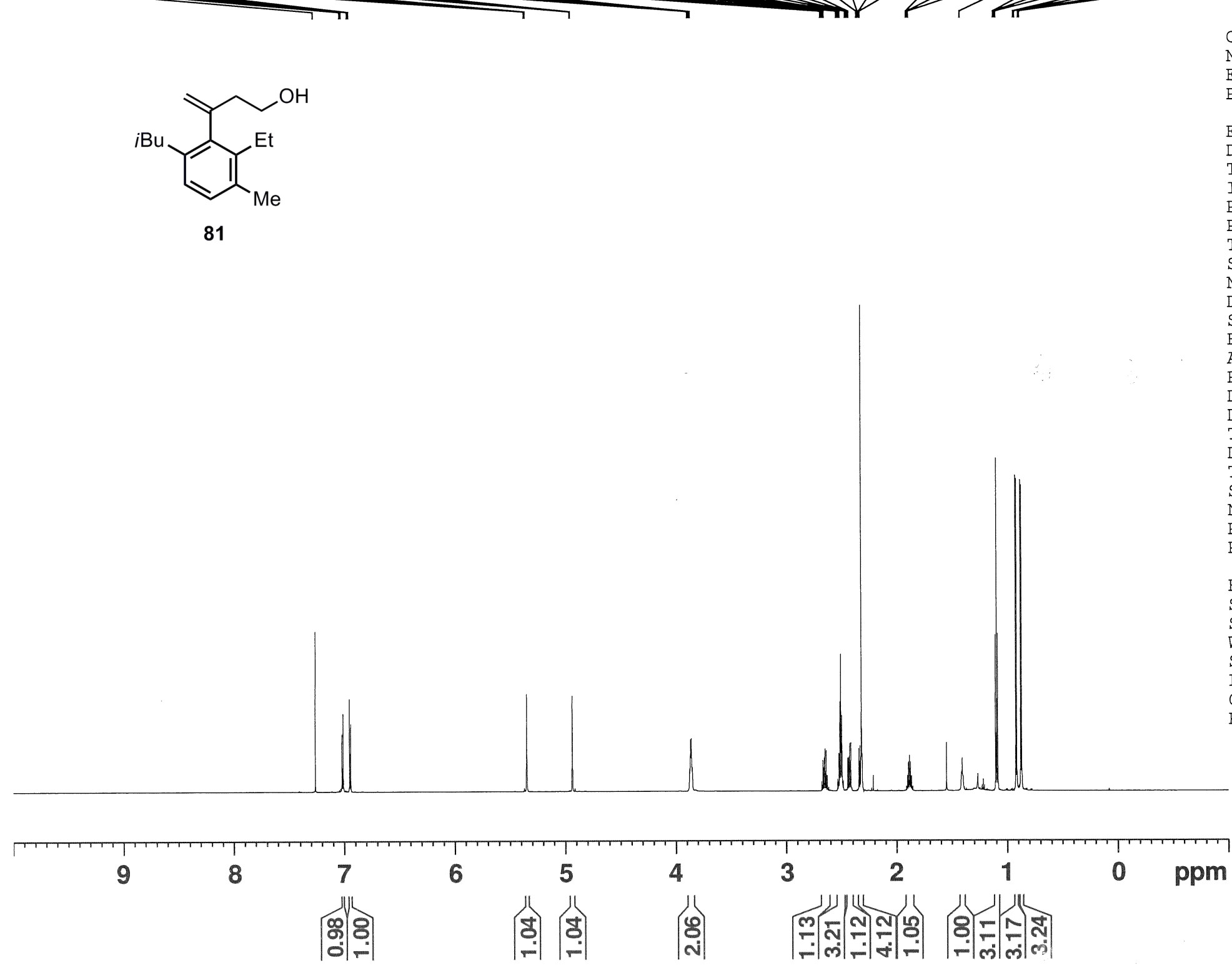

Current Data Parameters NAME

EXPNO

PROCNO $\mathrm{XZ}-3-193$

F - Acquisition Parameter

Date_ 20190606

Iime $18.00 \mathrm{~h}$

INSTRUM spect

PROBHD Z566801_0015

PULPROG

$\mathrm{zg} 30$

TD

SOLVENT

NS

SWH

AQ

RG

$D W$

DE

TE

TD 0

SFO1

NUC 1

1

PLW1

65536

$\mathrm{CDCl} 3$

16

$10504.202 \mathrm{~Hz}$

$0.320563 \mathrm{~Hz}$

$3.1195135 \mathrm{sec}$

128

47.600 usec

6.50 usec

$303.2 \mathrm{~K}$

$1.00000000 \mathrm{sec}$

$700.0115500 \mathrm{MHz}$ $1 \mathrm{H}$

12.88 usec

$40.00000000 \mathrm{~W}$

2 - Processing parameters

SI 131072

SF $\quad 700.0070164 \mathrm{MHz}$

$S$ SB

LB 0

$0 \mathrm{~Hz}$

no

C

1.00 


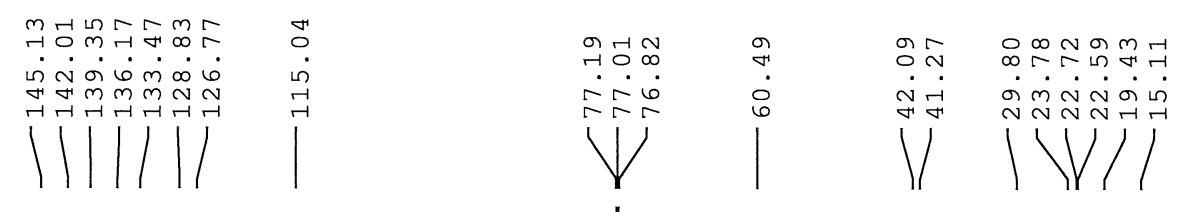

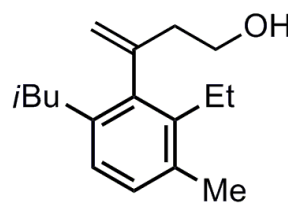

81

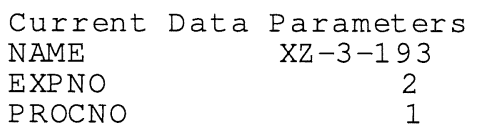

PROCNO

F2 - Acquisition Parameter Date_ 20190606

Time

INSTRUM

PROBHD

PULPROG zgpg30

Z566801 spect

TD

SOLVENT

NS

SWH

FIDRES

$\mathrm{AQ}$

RG

DW

DE

TE

D 11

TD 0

$\mathrm{SFO1}$

NUC1

P1

P LW1

$\mathrm{SFO2}$

NUC2

CPDPRG [ 2

PCPD 2

LLW12

655

192

192

$42613.637 \mathrm{~Hz}$

$1.300465 \mathrm{~Hz}$

$0.7689557 \mathrm{sec}$ 203

11.733 usec 6.50 usec $303.2 \mathrm{~K}$

$2.00000000 \mathrm{sec}$ $0.03000000 \mathrm{sec}$

$176.0362620 \mathrm{MHz}$ $13 \mathrm{C}$

8.95 usec

$100.00000000 \mathrm{~W}$

$700.0098000 \mathrm{MHz}$

$1 \mathrm{H}$
waltz 16

65.00 user

$40.00000000 \mathrm{~W}$

$1.57060003 \mathrm{~W}$

$0.79086000 \mathrm{~W}$

2 - Processing parameters

SI 131072

$\begin{array}{lcc}\text { SF } & & 176.0169002 \mathrm{MHz} \\ \text { WDW } & 0 & \text { EM } \\ \text { SSB } & 0 & 1.00 \mathrm{~Hz} \\ \text { LB } & 0 & \\ \text { GB } & 0 & 1.40\end{array}$

WDW

$S S B$

GB

P C

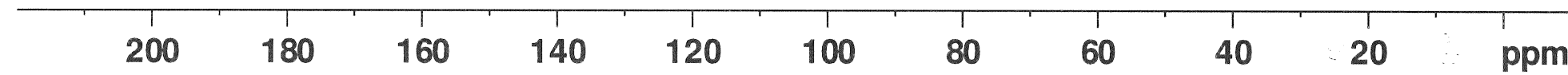




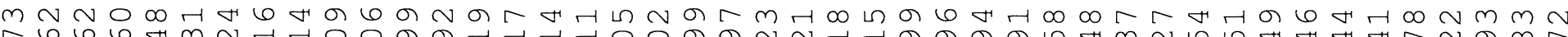
다

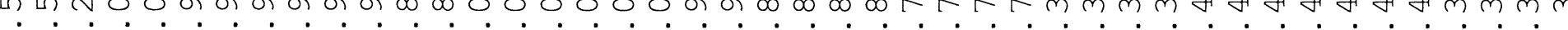

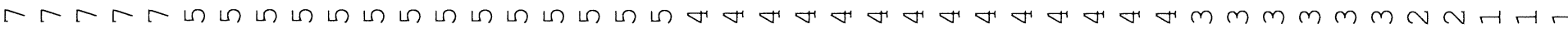
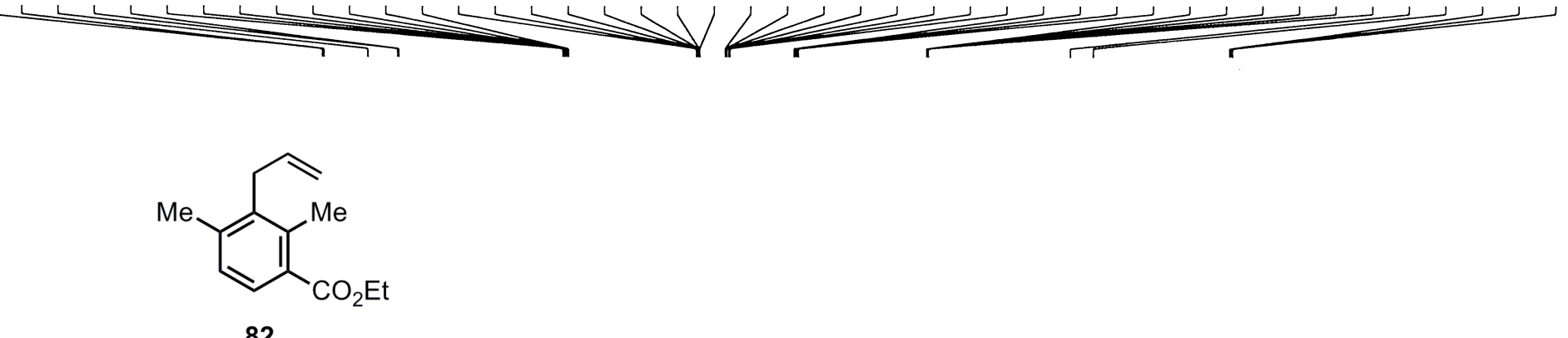

$$
\begin{array}{lr}
\text { Current } & \text { Data } \\
\text { NAME } & \mathrm{XZ}-3-189 \\
\text { EXPNO } & 4 \\
\text { PROCNO } & 1
\end{array}
$$

F2 - Acquisition Parameters

Date_ 20200709

Time $\quad 0.26 \mathrm{~h}$

82

2151340 spect

PUIPROG

$\begin{array}{lr}\text { TD } & \text { Z930 } \\ \text { TD } & 65536\end{array}$

SOLVENT CDCl3

NS

DS

SWH $\quad 10504.202 \mathrm{~Hz}$

$\begin{array}{lr}\text { FIDRES } & 0.320563 \mathrm{~Hz} \\ \mathrm{AQ} & 3.1195135 \mathrm{sec}\end{array}$

RG

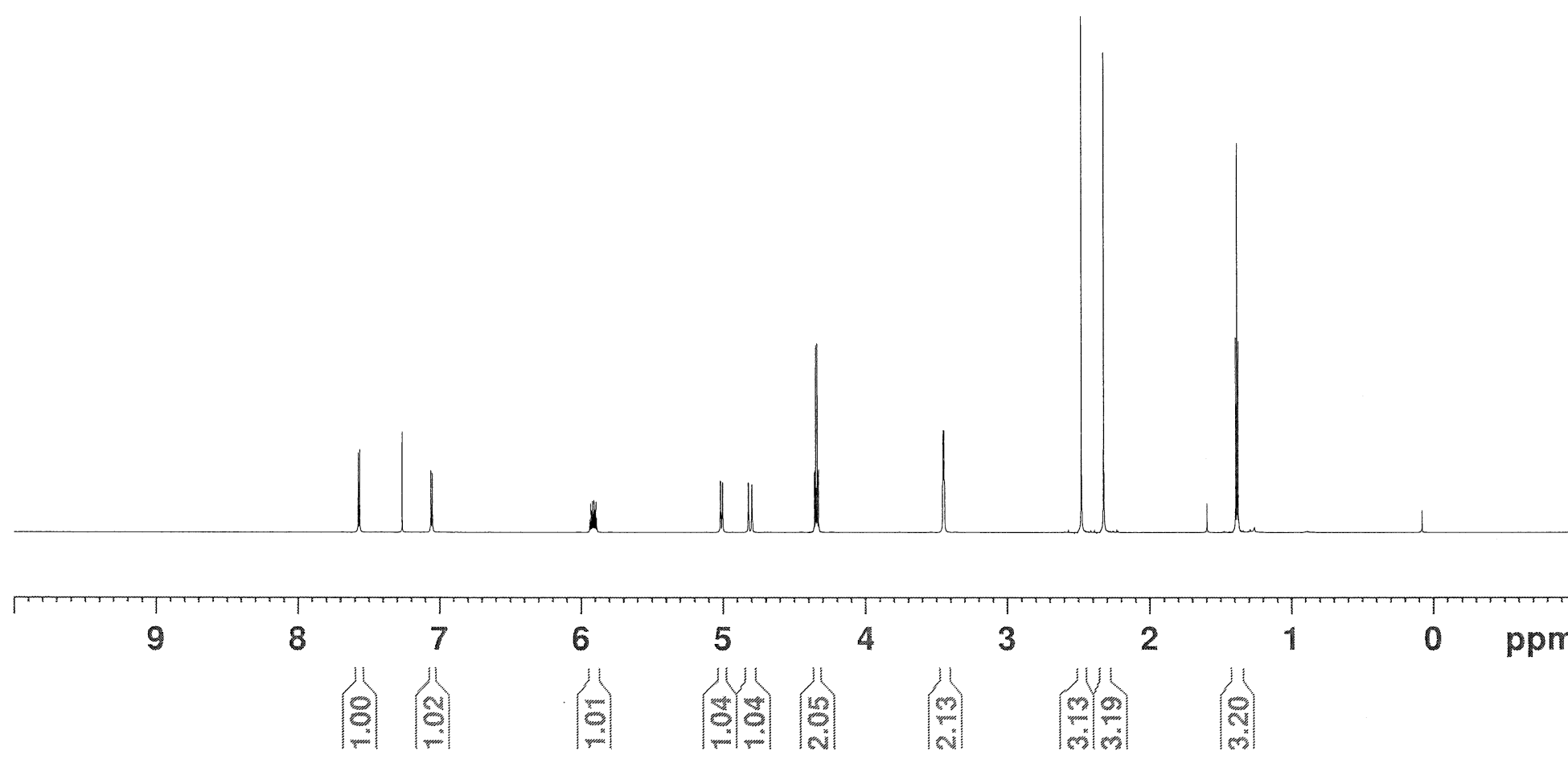

47.600 usec

$\begin{array}{lr}\mathrm{DE} & 20.00 \mathrm{usec}\end{array}$

$\begin{array}{lr}\mathrm{TE} & 303.2 \mathrm{~K} \\ \mathrm{DI} & 1.00000000 \mathrm{sec}\end{array}$

TD 0

SFO1 $\quad 700.0115500 \mathrm{MHz}$

0

P 1

PLW1

$1 \mathrm{H}$

3.17 usec

9.50 usec

F2 - Processing parameters

SI 131072

SF $\quad 700.0070146 \mathrm{MHz}$

WDW

LB

PC

EM
0
$0.30 \mathrm{~Hz}$
0
1.00




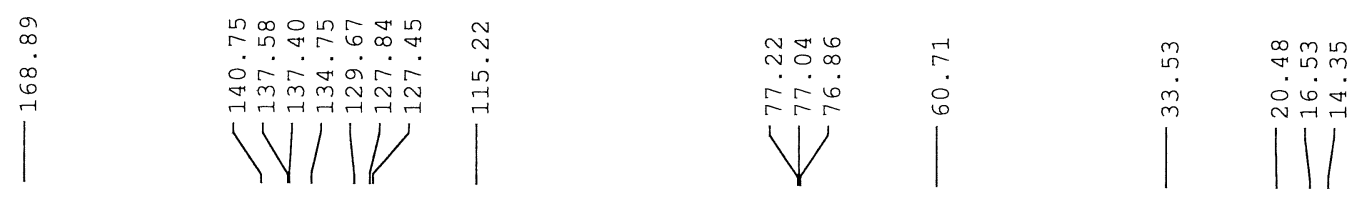

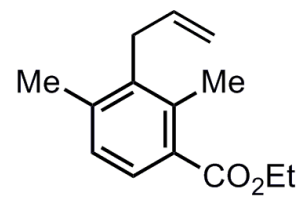

82
Current Data Parameters

NAME

EXPNO

PROCNO

F2 - Acquisition Parameters

Date_ 20200709

Time $\quad 0.30 \mathrm{~h}$

INSTRUM spect

PROBHD Z151340_0001)

PULPROG Zgpg30

TD 65536

SOLVENT CDCl3

DS

SWH

FIDRES

AQ

DW

$\mathrm{DE}$

TE

D 1

D 11

SFO1

NUC1

P 0

P 1

PLW1

$\mathrm{SFO} 2$

NUC2

CPDPRG [ 2

PCPD 2

PLW12

PLW13

22 - Processing parameters

SI 131072

SF $\quad 176.0169002 \mathrm{MHz}$

WDW

SSB

LB

GB
$\mathrm{EM}$

$1.00 \mathrm{~Hz}$

1.40

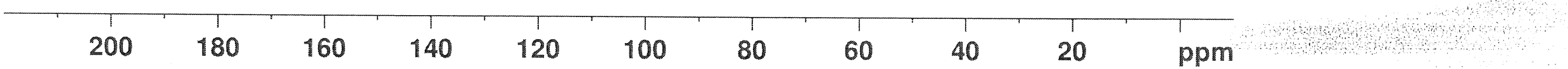




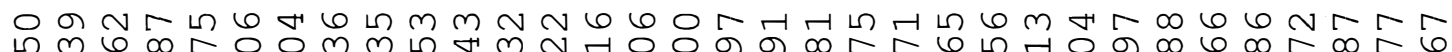

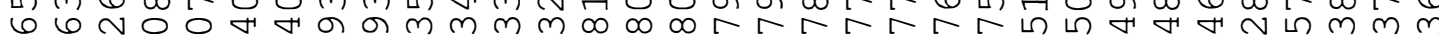

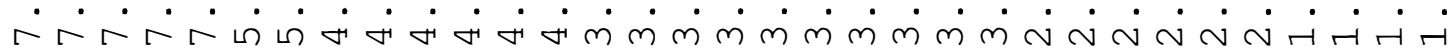

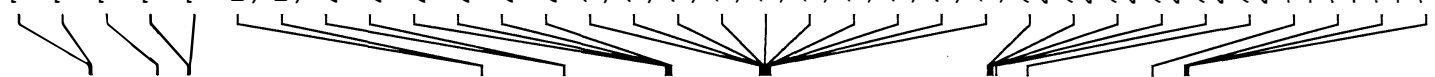

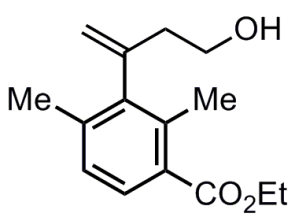

83

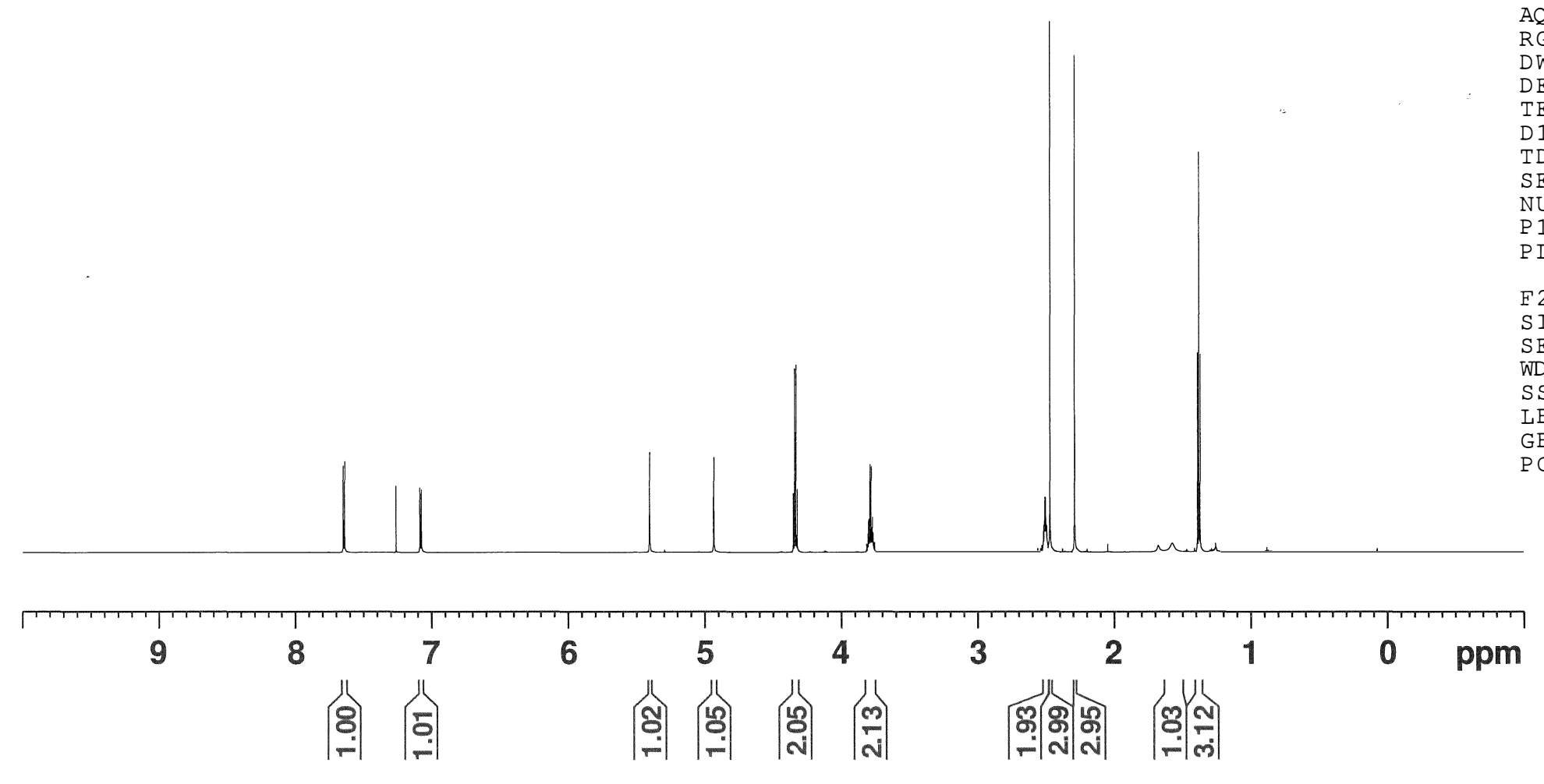

Current Data Parameters

NAME

EXPNO

PROCNO

$\mathrm{Xz}-3-188$

F2 - Acquisition Parameter

Date_ 20190602

Time

$16.10 \mathrm{~h}$

INSTRUM

PROBHD

PULPROG

Z151340_0001

TD

SOLVENT

NS

DS

FWH

$\mathrm{AQ}$

RG

DW

TE

T 1

$\mathrm{SFO1}$

NUC1

65536

CDC13

16

$10504.202 \mathrm{~Hz}$

$0.320563 \mathrm{~Hz}$

$3.1195135 \mathrm{sec}$ 5.6

47.600 usec 10.00 usec $303.2: \mathrm{K}$

$1.00000000 \mathrm{sec}$ $700.0115500 \mathrm{MHz}$ 9.50 usec

$13.00000000 \mathrm{~W}$

2 - Processing parameters SI 131072 $\quad 700.0070164 \mathrm{MHz}$ WDW no SSB $\mathrm{LB} \quad \mathrm{OHz}$

GB $\quad 0$

0

1.00 


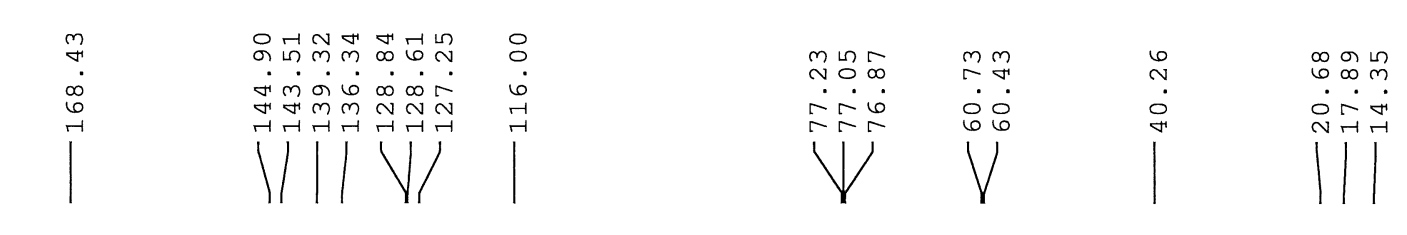

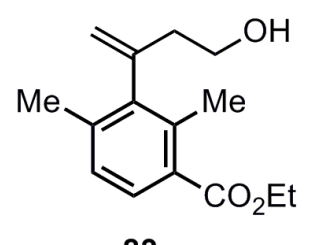

83

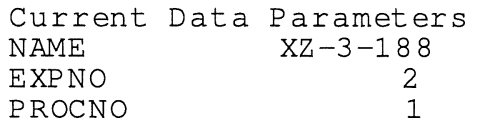

F2 - Acquisition Parameter Date_ 20190602 Time $\quad 16.16 \mathrm{~h}$ INSTRUM spect PROBHD Z151340_0001 PULPROG Z $\quad$ gpg30 TD 65536 SOLVENT CDCl3 $\begin{array}{lr}\text { NS } & 35\end{array}$ SWH FIDRES $A Q$

RG

DW

$\mathrm{DE}$

D 1

D 11
TD 0

$\mathrm{SFO} 1$

NUC 1

$\mathrm{P} 1$

P LW1

$\mathrm{SFO} 2$

$\mathrm{NUC} 2$

CPDPRG [ 2

PCPD 2

PLW2

P LW1 2

$42613.637 \mathrm{~Hz}$ $1.300465 \mathrm{~Hz}$ $0.7689557 \mathrm{sec}$ 203

11.733 usec 18.00 usec $303.2 \mathrm{~K}$

$2.00000000 \mathrm{sec}$ $0.03000000 \mathrm{sec}$

1.0300 $176.0362620 \mathrm{MHz}$ $13 \mathrm{C}$

11.25 usec $29.00000000 \mathrm{~W}$ $700.0098000 \mathrm{MHz}$

$$
\begin{gathered}
1 \mathrm{H} \\
\text { waltz } 16
\end{gathered}
$$$$
65.00 \text { use }
$$

$13.00000000 \mathrm{~W}$

$0.27768999 \mathrm{~W}$

$0.13982999 \mathrm{~W}$

F2 - Processing parameters
SI

SF $\quad 176.0169002 \mathrm{MHz}$ WDW EM

SSB $\quad 0$

LB

GB

PC

$1.00 \mathrm{~Hz}$

1.40

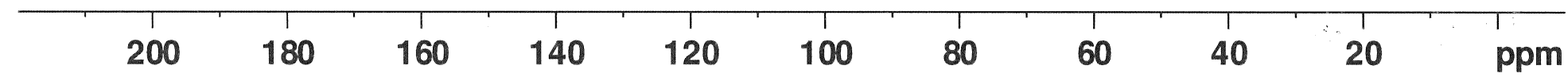


$m \sim N \pi$ M m

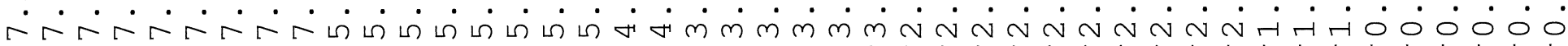

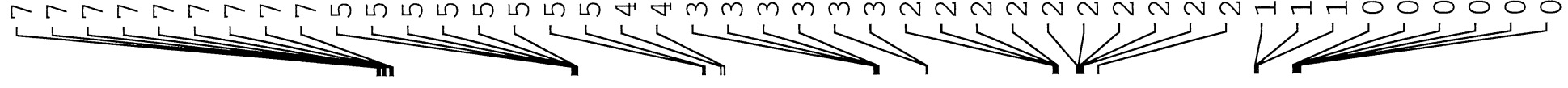

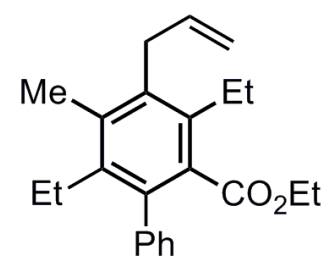

84

\section{Current Data Parameters NAME EXPNO $\mathrm{XZ}-3-230$ PROCNO}

F2 - Acquisition Parameter Date_ 20190708 Time

INSTRUM

PROBHD

PULPROG

TD

SOLVENT

NS

DS

FIDRES

AQ

RG

DW

DE

TE

TD 0

SFO1

NUC1

P 1

PLW1 151340 spect Z151340_0001 $\mathrm{zg} 30$ CDC 13 16

$10504.202 \mathrm{~Hz}$ $0.320563 \mathrm{~Hz}$ $3.1195135 \mathrm{sec}$ 20.2

47.600 usec 20.00 usec $303.4 \mathrm{~K}$

$1.00000000 \mathrm{sec}$ $700.0115500 \mathrm{MHz}$$$
9.50 \text { usec }
$$

$13.00000000 \mathrm{~W}$

- Processing parameters SI 131072

SF $\quad 700.0070167 \mathrm{MHz}$

SSB

0

no

$0 \mathrm{~Hz}$

GB

1.00

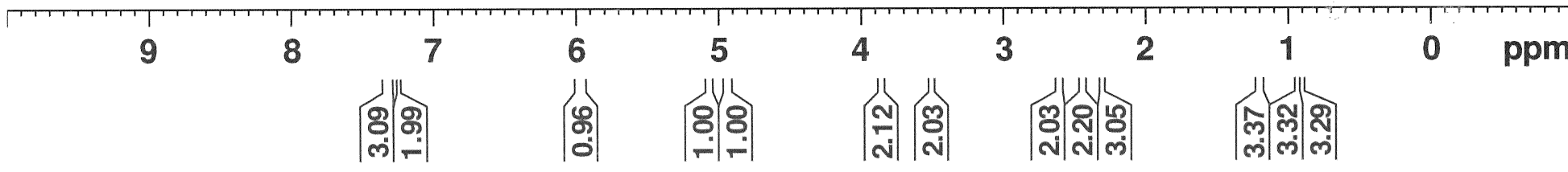




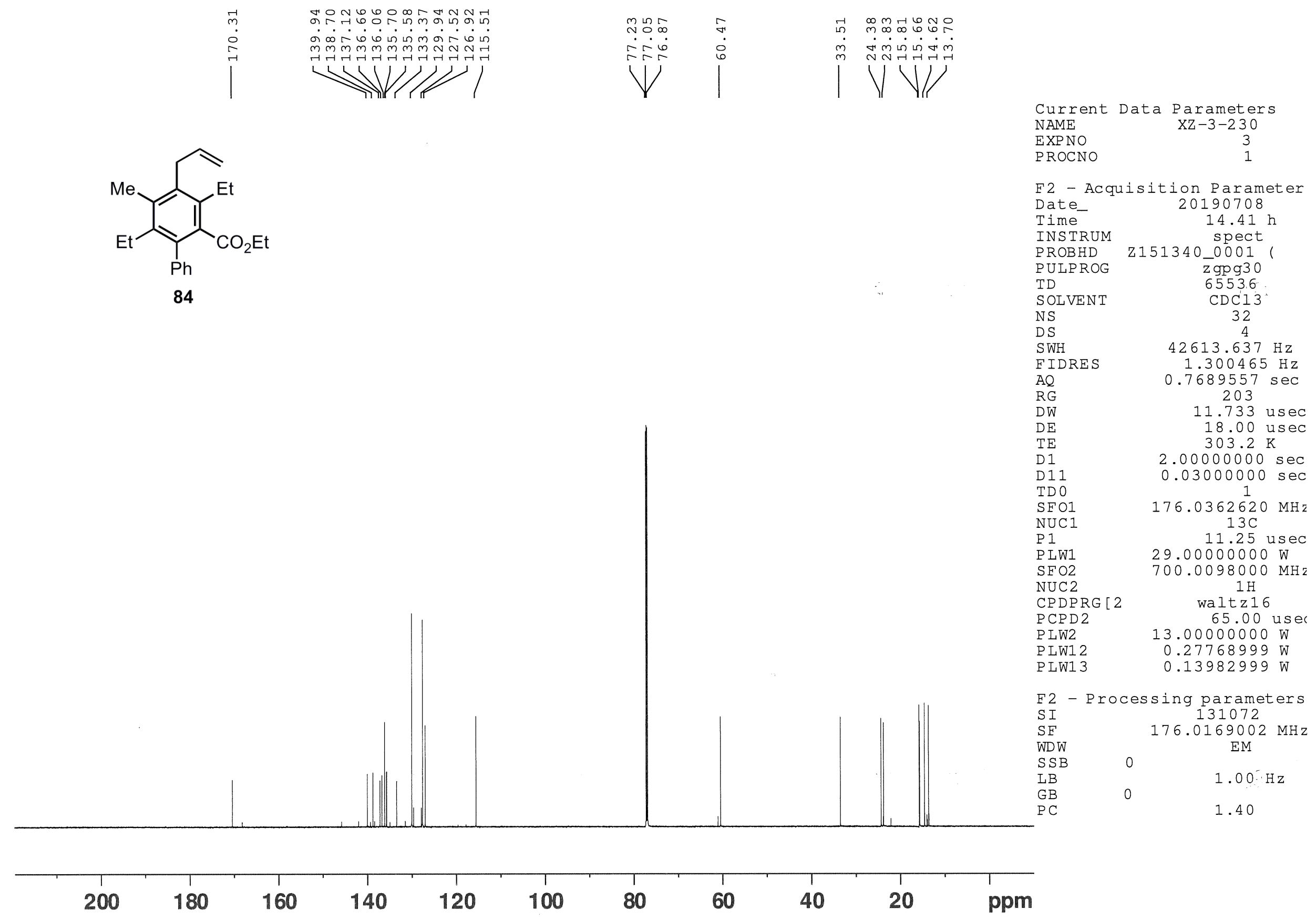




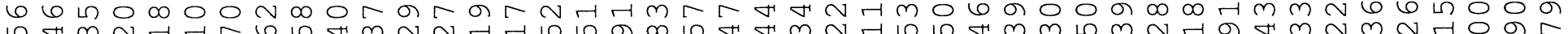

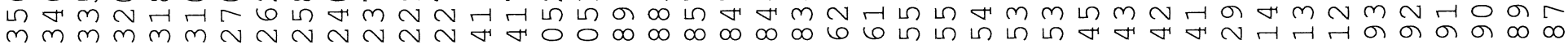

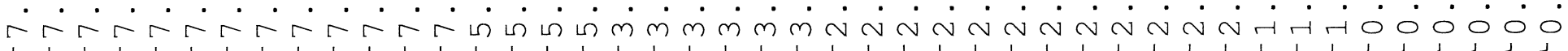

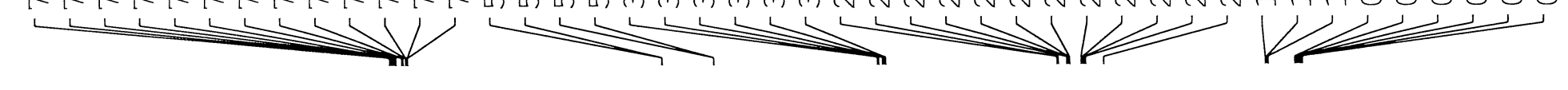

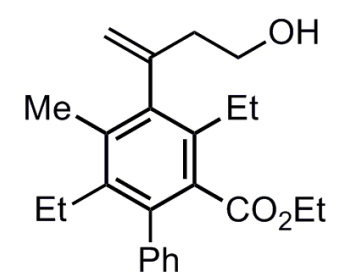

85
Current Data Parameters NAME

EXPNO
PROCNO

F2 - Acquisition Parameters

Date__ 20190919

Time $\quad 10.35 \mathrm{~h}$

INSTRUM spect

PROBHD Z151340_0001 (

PULPROG Z930

$\begin{array}{lr}\text { PULPROG } & \text { Zg30 } \\ \text { TD } & 65536\end{array}$

$\begin{array}{ll}\text { TD } & 65536 \\ & \text { CDCl3 }\end{array}$

NS

SWH

FIDRES

AQ
RG
DW

DW

$\mathrm{DE}$

TE

D1 0

$\mathrm{SFO} 1$

NUC1

P 0
P1
P LW1

2
$10504.202 \mathrm{~Hz}$

$0.320563 \mathrm{~Hz}$

$3.1195135 \mathrm{sec}$

47.600 usec

20.00 usec

$303.2 \mathrm{~K}$

$1.00000000 \mathrm{sec}$

$1.00000000 \mathrm{sec}$

$700.0115500 \mathrm{MHz}$ $1 \mathrm{H}$

3.17 usec

9.50 usec

$13.00000000 \mathrm{~W}$

F2 - Processing parameters SI 131072

SF $\quad 700.0070168 \mathrm{MHz}$

WDW

SSB

$L B$

PB

no

.00

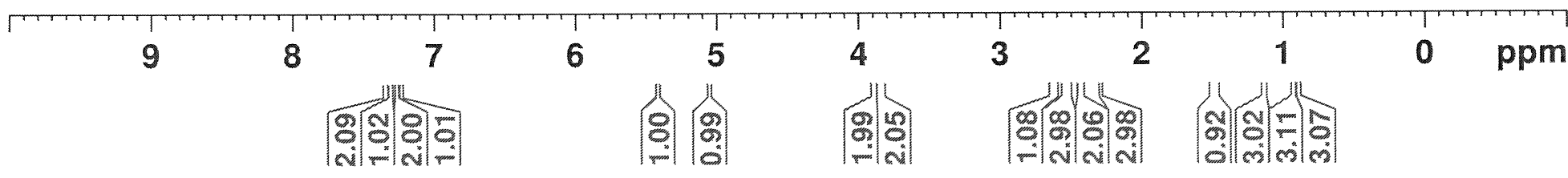



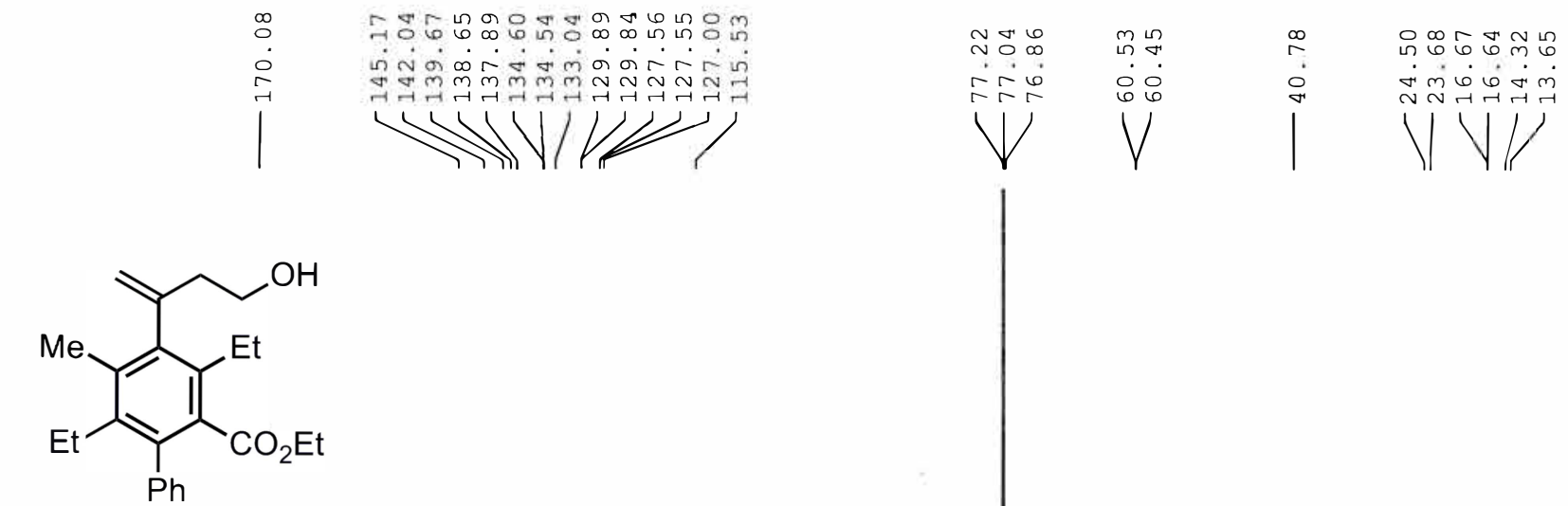

NAM

Current Data Parameters

EXPNO

PROCNO

$\mathrm{XZ}-4-12$

F2 - Acquisition Parameters

Date_ 20190919

Time 10.55 h

INSTRUM spect

PROBHD Z151340_0001

PULPROG ZgPg30

SOLVENT

zgpg 30
65536

85

$$
\text { NS }
$$

SWH

$42613.637 \mathrm{~Hz}$

FIDRES $\quad 1.300465 \mathrm{~Hz}$

AQ

RW

DW

$\mathrm{DE}$

DE
D 11
TD

TD 0

$\mathrm{SFO} 1$

NUC1

PO

P1

P LW1

$\mathrm{SFO} 2$

CPDPRG [2

PCPD 2

PCPD2

PLW12

PLW13

$0.7689557 \mathrm{sec}$

$$
\begin{array}{r}
203 \\
11.733
\end{array}
$$

11.733 usec

18.00 usec

$303.2 \mathrm{~K}$

$2.00000000 \mathrm{sec}$

$0.03000000 \mathrm{sec}$

$176.0362620 \mathrm{MHz}$

$$
13 \mathrm{C}
$$

3.75 usec

11.25 use

$29.00000000 \mathrm{~W}$

$700.0098000 \mathrm{MHz}$

$$
\begin{array}{r}
1 \mathrm{H} \\
\text { waltzi6 }
\end{array}
$$$$
65.00 \text { use }
$$

$13.00000000 \mathrm{~W}$

$13.00000000 \mathrm{~W}$
$0.27768999 \mathrm{~W}$

$0.13982999 \mathrm{~W}$

F2 - Processing parameters SI $\quad 131072$

SF $\quad 176.0169002 \mathrm{MHz}$

WDW

SSB

LB

$$
\begin{gathered}
\text { EM } \\
0 \\
1.00 \mathrm{~Hz} \\
0 \\
1.40
\end{gathered}
$$

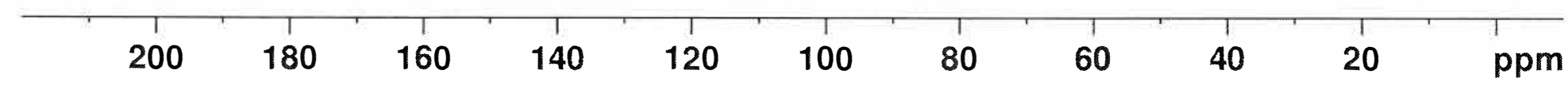


Zhang, 1_1 mix, S1714B, Beaudry, 091819BY-17

100

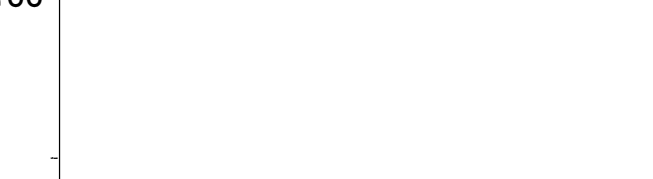

UCA138

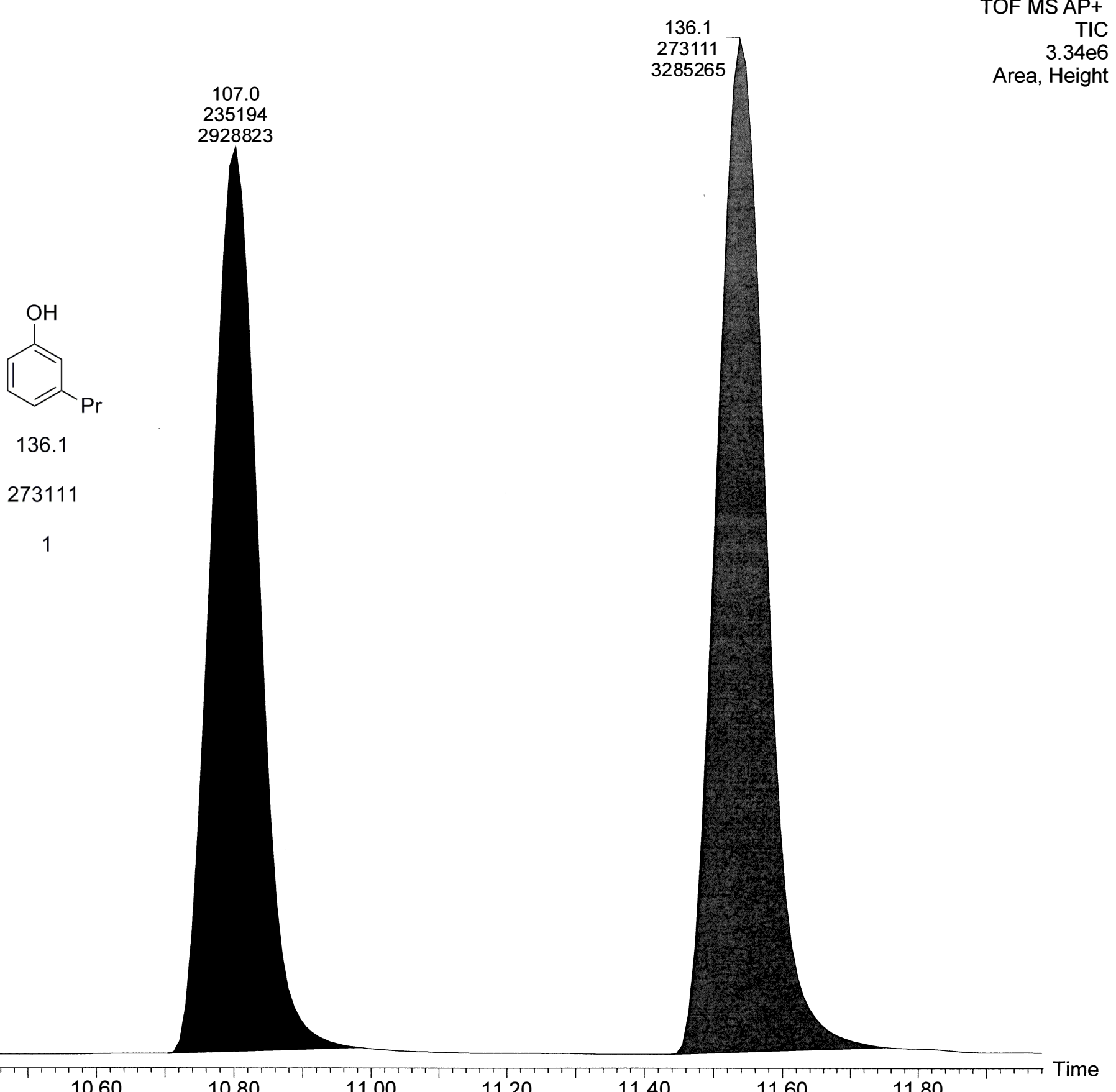

10.80
Height
Standard Experiment

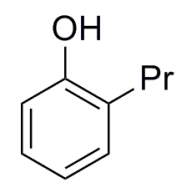

Time $=107.0$

Area $=235194$

Ratio $=1$

1 
Zhang, XZ-3-15-X-crude, S1714B, Beaudry 091719BY-04

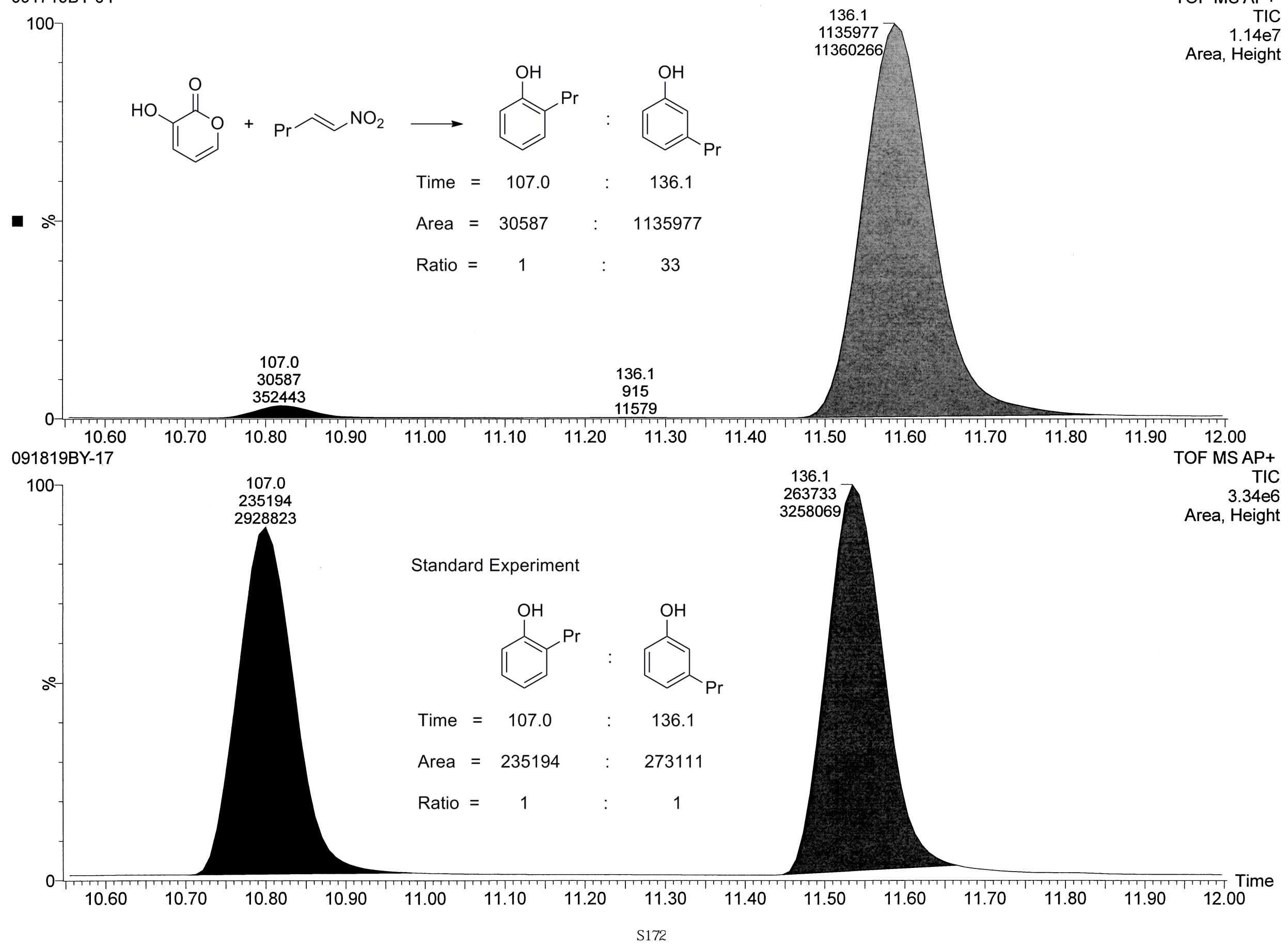




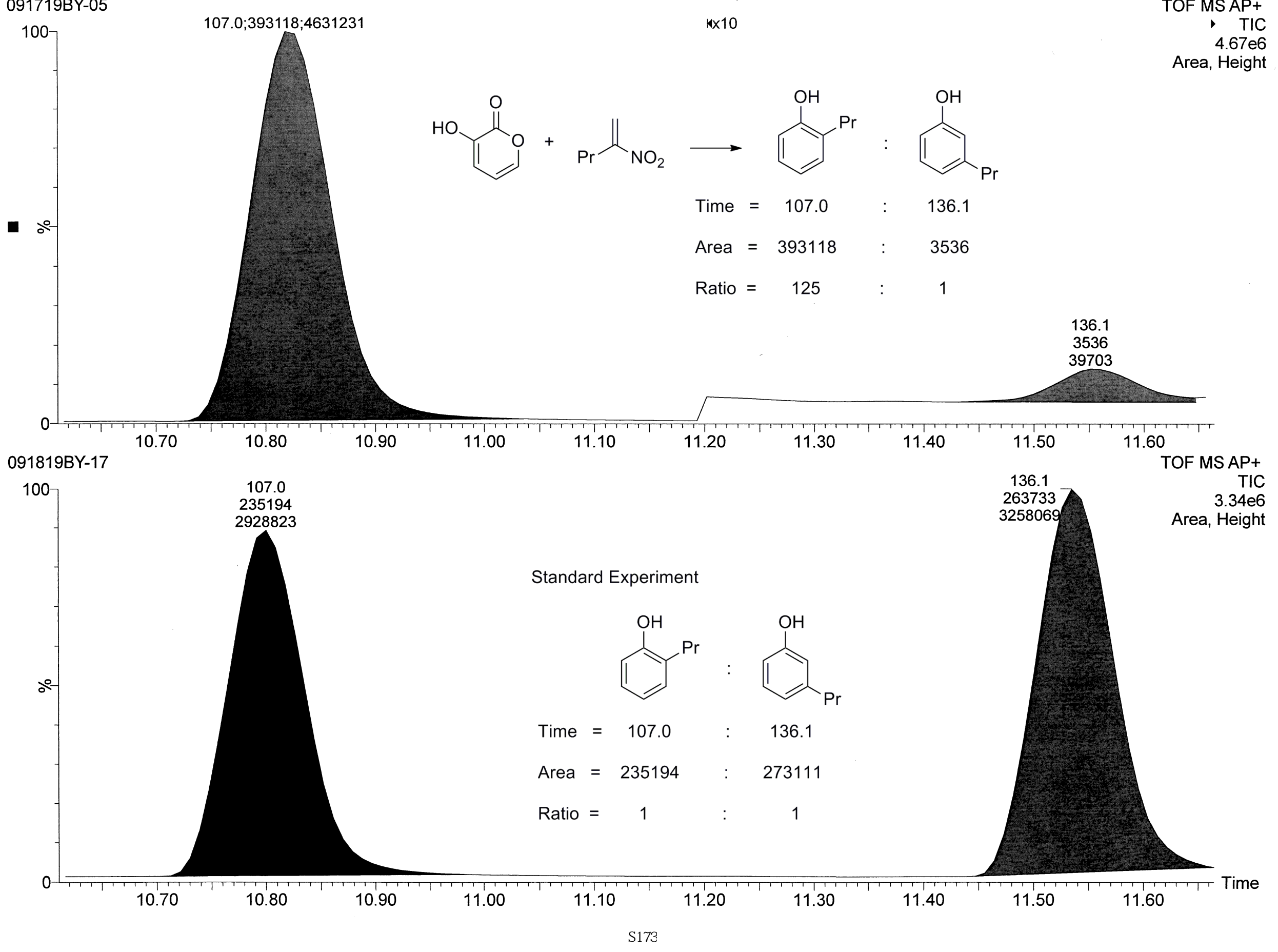

